University of Tennessee Health Science Center UTHSC Digital Commons

$12-2012$

\title{
Nanotherapies for Treating Prostate Cancer
}

Michael Danquah

University of Tennessee Health Science Center

Follow this and additional works at: https://dc.uthsc.edu/dissertations

Part of the Pharmaceutics and Drug Design Commons

\section{Recommended Citation}

Danquah, Michael , "Nanotherapies for Treating Prostate Cancer" (2012). Theses and Dissertations (ETD). Paper 58. http://dx.doi.org/10.21007/etd.cghs.2012.0062.

This Dissertation is brought to you for free and open access by the College of Graduate Health Sciences at UTHSC Digital Commons. It has been accepted for inclusion in Theses and Dissertations (ETD) by an authorized administrator of UTHSC Digital Commons. For more information, please contact jwelch30@uthsc.edu. 


\title{
Nanotherapies for Treating Prostate Cancer
}

\begin{abstract}
Current prostate cancer treatment remains ineffective primarily due to ineffectual therapeutic strategies and numerous tumor-associated physiological barriers which hinder efficacy of anticancer agents. Therefore, the focus of this study was to investigate a new combination therapy approach for treating prostate cancer and develop polymeric nanocarriers to facilitate anticancer drug and nucleic acid delivery.
\end{abstract}

It was hypothesized that simultaneously targeting androgen-androgen receptor (AR) and X-linked inhibitor of apoptosis protein (XIAP) signaling pathways would be effective in treating prostate cancer. The effect of bicalutamide (antiandrogen) and embelin (XIAP inhibitor) on the growth of prostate cancer cells in vitro and in vivo was first examined. Embelin induced caspase 3 and 9 activation in LNCaP and C4-2 cells by decreasing XIAP expression and was more potent than bicalutamide in killing prostate tumor cells irrespective of their androgen status. Using a combination of MTT assay and isobologram analyses, combination of bicalutamide and embelin was observed to be cell line and schedule dependent. Since bicalutamide and embelin are extremely hydrophobic, polymeric micelles were fabricated using polyethylene glycol-bpolylactic acid (PEG-b-PLA) copolymer to improve drug solubility. Micellar formulations were found to result in at least 60 -fold increase in the aqueous solubility of bicalutamide and embelin. Tumor growth was also effectively regressed upon treatment with bicalutamide, but the extent of tumor regression was significantly higher when bicalutamide was formulated in micelles.

To further improve bicalutamide aqueous solubility, a series of novel biodegradable copolymers for the systematic micellar delivery of bicalutamide was designed and synthesized. Flory-Huggins interaction parameter $(\mathrm{XHH})$ was used to assess compatibility between bicalutamide and poly (L-lactide) or poly (carbonate-co-lactide) polymer pairs. Polyethylene glycol-b-poly (carbonate-co-lactide) [PEG-b-P(CB-coLA)] copolymers were synthesized and characterized by NMR and gel permeation chromatography. Micelles formulated using these copolymers had average diameter of $100 \mathrm{~nm}$ and distinct spherical shape. Drug loading studies revealed that adding the carbonate monomer could increase bicalutamide loading. Among the series, drug loading of micelles formulated with PEG-b-P(CB-co-LA) copolymer containing $20 \mathrm{~mol} \%$ carbonate was about four-fold higher than PEG-b-PLLA and aqueous solubility of bicalutamide increased from 5 to $4000 \mu \mathrm{g} / \mathrm{mL}$. CMC values for PEG-b-P(CB-Co-LA) copolymers was up to 10-fold lower than those of PEG-b-PLLA. Bicalutamide-loaded PEG-bP(CB-co-LA) micelles showed significant inhibition of LNCaP cell growth in a dose dependent manner which was similar to the methanol solution of free drug.

Bicalutamide tends to act as an agonist rather than an antagonist after prolonged treatment. Hence, a second generation antiandrogen ((S)-N-(4-cyano-3-(trifluoromethyl) phenyl)-3-((4-cyanophenyl)(methyl)amino)-2-hydroxy-2-methylpropanamide) (CBDIV17)) was synthesized and its effect in combination with XIAP inhibitors for treating advanced prostate cancer was determined. CBDIV17 was more potent than bicalutamide and inhibited proliferation of C4-2 and LNCaP cells. CBDIV17-induced apoptosis more effectively compared to bicalutamide and significantly inhibited DNA replication. Combination of CBDIV17 and embelin resulted in supra-additive antiproliferative and apoptotic effects. Embelin downregulated AR expression and decreased androgen-mediated AR phosphorylation at Ser81. These hydrophobic drugs were solubilized using micelles prepared using polyethylene glycol-b-poly (carbonate-co-lactide) (PEG-b-p(CB-co-PLA)) copolymer. Combination therapy inhibited prostate tumor growth more effectively compared to control or monotherapy in vivo.

Polymeric micelles tend to be unstable in vivo. To address this, lactic acid- and carbonate-based biodegradable core- and core-corona crosslinkable copolymers for anticancer drug delivery were synthesized and evaluated. Methoxy poly (ethylene glycol)-b-poly (carbonate-co-lactide- 
co-5-methyl-5-allyloxycarbonyl-1,3-dioxane-2-one) [mPEG-b-P(CB-coLA-co-MAC)] and methoxy poly (ethylene glycol)-b-poly(acryloyl carbonate)-b-poly (carbonate-co-lactide) [mPEG-b-PMAC-b-P(CB-co-LA)] copolymers were synthesized by ring opening polymerization of $L A, C B$ and MAC using $\mathrm{MPEG}$ as an macroinitiator and 1,8- diazabicycloundec-7-ene (DBU) as a catalyst. These amphiphilic copolymers which exhibited low polydispersity (1.08) and CMC values $(0.8-1 \mathrm{mg} / \mathrm{L})$ were used to prepare micelles with or without drug and stabilized by crosslinking via radical polymerization of double bonds introduced in the core and interface to improve stability. $1 \mathrm{H}$ NMR and IR spectroscopy confirmed successful crosslinking (crosslinking efficiency of $\sim 70 \%$ ) while light scattering and transmission electron microscopy were used to determine micelle size and morphology. Crosslinked micelles demonstrated enhanced stability against extensive dilution with aqueous solvents and in the presence of physiological simulating serum concentration.

Since nucleic acids are not susceptible to chemoresistance, it was determined whether simultaneous AR and XIAP gene silencing via RNA interference (RNAi) has the potential to treat hormone refractory prostate cancer. Small interfering RNAs (siRNAs) targeting three different regions of AR and XIAP were screened by transfecting $\mathrm{LNCaP}$ and $\mathrm{C} 4-2$ prostate cancer cells and their silencing effects determined at mRNA and protein levels by real time RTPCR, Western blot and ELISA. The most potent siRNAs against $A R$ and XIAP were selected and their combination was more effective in inducing apoptosis and inhibiting C4-2 cell proliferation. $\mathrm{N}$-(2-hydroxypropyl) methacylamide (HPMA) based copolymer containing polycationic, lipid and pka modulator moieties was developed for delivering the most potent siRNA and bipartite plasmid encoding two different shRNAs targeting AR and XIAP. HPMA based copolymer showed efficient cellular uptake in prostate cancer cells.

The findings reported in this work demonstrate the potential benefit of combination therapy targeting AR and XIAP pathways for treating prostate cancer using polymeric nanocarriers. Systematic chemical tailoring of polymers for improving drug loading as well as efforts made to improve micelle stability through crosslinking also generated insights which can be applied to other drugs and cancer types. Results reported in this study therefore offer a new way of addressing the time old problem of treating cancer.

\section{Document Type}

Dissertation

Degree Name

Doctor of Philosophy (PhD)

Program

Pharmaceutical Sciences

Research Advisor

Ram I. Mahato, Ph.D.

\section{Keywords}

Androgen Receptor, Biomaterials. Drug Delivery, Polymeric Micelles, Prostate Cancer, XIAP

\section{Subject Categories}

Medicine and Health Sciences | Pharmaceutics and Drug Design | Pharmacy and Pharmaceutical Sciences 


\title{
NANOTHERAPIES FOR TREATING PROSTATE CANCER
}

\author{
A Dissertation \\ Presented for \\ The Graduate Studies Council \\ The University of Tennessee \\ Health Science Center
}

\begin{abstract}
In Partial Fulfillment
Of the Requirements for the Degree

Doctor of Philosophy

From The University of Tennessee
\end{abstract}

By

Michael Danquah

December 2012 
Portions of Chapter 2 (C) 2011 by Elsevier.

Portions of Chapter 3 (C) 2009 by Springer.

Portions of Chapter 4 (C) 2010 by Elsevier.

Portions of Chapter 5 (c) 2012 by Springer.

Portions of Chapter 6 (C) 2012 by John Wiley \& Sons.

All other material (C) 2012 by Michael Danquah.

All rights reserved. 


\section{DEDICATION}

To my parents: George and Elizabeth, with heartfelt love and gratitude. I am where I am because of your sweat, tears, and sacrifices. Thank you for teaching me to believe in academic excellence.

To my brother Gabriel: for the priceless lessons your remarkable life and graceful death has taught me. In the vastness of space and immensity of time I am glad to have shared this planet with you. Although you are gone you are not forgotten. I'll see you again.

To Jennifer: for numerous reasons she knows so well. 


\section{ACKNOWLEDGEMENTS}

I gladly share credit for the successful execution and completion of the work reported in this dissertation with many great souls. To concretize the ideas mentioned here required some mental giants pointing me in the right direction and walking with me along uncharted paths. Yet still other persons simply made it impossible for me to quit even if I wanted to. I count myself fortunate to have had such people throughout this undertaking. To them, I am eternally grateful and forever indebted.

In particular, I would like to express my sincere and profound gratitude to my mentor Dr. Ram I. Mahato for his guidance and support. I also thank him for being a powerful example of what it takes to be a world-class researcher and most of all for the countless opportunities he made available for me to develop, refine and expand my skills as a scientist.

Special thanks to my committee members: Dr. Duane D. Miller, Dr. Xing Zhang, Dr. Sarka Beranova and Dr. Tomoko Fujiwara for their precious suggestions and priceless assistance.

I would also like to thank all members of the Mahato lab, past and present for their comradeship. I am also grateful to my collaborators, especially Dr. Charles Duke, Dr. Renukadevi Patil, Dr. Yan Lu and Davita Watkins for their help in various ways and at different times.

My deepest appreciation to my family: I could not have completed my doctoral study or this dissertation without their unqualified love and support.

Finally, I would like to thank God for being my source of inspiration and strength in trying times. I have found there is such a thing as peace which surpasses all understanding. 


\begin{abstract}
Current prostate cancer treatment remains ineffective primarily due to ineffectual therapeutic strategies and numerous tumor-associated physiological barriers which hinder efficacy of anticancer agents. Therefore, the focus of this study was to investigate a new combination therapy approach for treating prostate cancer and develop polymeric nanocarriers to facilitate anticancer drug and nucleic acid delivery.
\end{abstract}

It was hypothesized that simultaneously targeting androgen-androgen receptor (AR) and X-linked inhibitor of apoptosis protein (XIAP) signaling pathways would be effective in treating prostate cancer. The effect of bicalutamide (antiandrogen) and embelin (XIAP inhibitor) on the growth of prostate cancer cells in vitro and in vivo was first examined. Embelin induced caspase 3 and 9 activation in LNCaP and C4-2 cells by decreasing XIAP expression and was more potent than bicalutamide in killing prostate tumor cells irrespective of their androgen status. Using a combination of MTT assay and isobologram analyses, combination of bicalutamide and embelin was observed to be cell line and schedule dependent. Since bicalutamide and embelin are extremely hydrophobic, polymeric micelles were fabricated using polyethylene glycol-b-polylactic acid

(PEG-b-PLA) copolymer to improve drug solubility. Micellar formulations were found to result in at least 60 -fold increase in the aqueous solubility of bicalutamide and embelin. Tumor growth was also effectively regressed upon treatment with bicalutamide, but the extent of tumor regression was significantly higher when bicalutamide was formulated in micelles.

To further improve bicalutamide aqueous solubility, a series of novel biodegradable copolymers for the systematic micellar delivery of bicalutamide was designed and synthesized. Flory-Huggins interaction parameter $\left(\chi_{\mathrm{FH}}\right)$ was used to assess compatibility between bicalutamide and poly (L-lactide) or poly (carbonate-co-lactide) polymer pairs. Polyethylene glycol-b-poly (carbonate-co-lactide) [PEG-b-P(CB-co-LA)] copolymers were synthesized and characterized by NMR and gel permeation chromatography. Micelles formulated using these copolymers had average diameter of $100 \mathrm{~nm}$ and distinct spherical shape. Drug loading studies revealed that adding the carbonate monomer could increase bicalutamide loading. Among the series, drug loading of micelles formulated with PEG-b-P(CB-co-LA) copolymer containing $20 \mathrm{~mol} \%$ carbonate was about four-fold higher than PEG-b-PLLA and aqueous solubility of bicalutamide increased from 5 to $4000 \mu \mathrm{g} / \mathrm{mL}$. CMC values for PEG-b-P(CB-co-LA) copolymers was up to 10-fold lower than those of PEG-b-PLLA. Bicalutamide-loaded PEG-b-P(CB-co-LA) micelles showed significant inhibition of $\mathrm{LNCaP}$ cell growth in a dose dependent manner which was similar to the methanol solution of free drug.

Bicalutamide tends to act as an agonist rather than an antagonist after prolonged treatment. Hence, a second generation antiandrogen ((S)-N-(4-cyano-3-(trifluoromethyl) phenyl)-3-((4-cyanophenyl)(methyl)amino)-2-hydroxy-2-methylpropanamide) (CBDIV17)) was synthesized and its effect in combination with XIAP inhibitors for treating advanced prostate cancer was determined. CBDIV17 was more potent than 
bicalutamide and inhibited proliferation of C4-2 and LNCaP cells. CBDIV17-induced apoptosis more effectively compared to bicalutamide and significantly inhibited DNA replication. Combination of CBDIV17 and embelin resulted in supra-additive antiproliferative and apoptotic effects. Embelin downregulated AR expression and decreased androgen-mediated AR phosphorylation at $\mathrm{Ser}^{81}$. These hydrophobic drugs were solubilized using micelles prepared using polyethylene glycol-b-poly (carbonate-colactide) (PEG-b-p(CB-co-PLA)) copolymer. Combination therapy inhibited prostate tumor growth more effectively compared to control or monotherapy in vivo.

Polymeric micelles tend to be unstable in vivo. To address this, lactic acid- and carbonate-based biodegradable core- and core-corona crosslinkable copolymers for anticancer drug delivery were synthesized and evaluated. Methoxy poly (ethylene glycol)- $b$-poly (carbonate-co-lactide-co-5-methyl-5-allyloxycarbonyl-1,3-dioxane-2-one) [mPEG- $b$-P(CB-co-LA-co-MAC)] and methoxy poly (ethylene glycol)-b-poly(acryloyl carbonate)- $b$-poly (carbonate-co-lactide) [mPEG- $b$-PMAC- $b$-P(CB-co-LA)] copolymers were synthesized by ring opening polymerization of LA, CB and MAC using $\mathrm{mPEG}$ as an macroinitiator and 1,8-diazabicycloundec-7-ene (DBU) as a catalyst. These amphiphilic copolymers which exhibited low polydispersity (1.08) and CMC values $(0.8-1 \mathrm{mg} / \mathrm{L})$ were used to prepare micelles with or without drug and stabilized by crosslinking via radical polymerization of double bonds introduced in the core and interface to improve stability. ${ }^{1} \mathrm{H}$ NMR and IR spectroscopy confirmed successful crosslinking (crosslinking efficiency of $\sim 70 \%$ ) while light scattering and transmission electron microscopy were used to determine micelle size and morphology. Crosslinked micelles demonstrated enhanced stability against extensive dilution with aqueous solvents and in the presence of physiological simulating serum concentration.

Since nucleic acids are not susceptible to chemoresistance, it was determined whether simultaneous AR and XIAP gene silencing via RNA interference (RNAi) has the potential to treat hormone refractory prostate cancer. Small interfering RNAs (siRNAs) targeting three different regions of AR and XIAP were screened by transfecting LNCaP and C4-2 prostate cancer cells and their silencing effects determined at mRNA and protein levels by real time RT-PCR, Western blot and ELISA. The most potent siRNAs against $\mathrm{AR}$ and XIAP were selected and their combination was more effective in inducing apoptosis and inhibiting C4-2 cell proliferation. N-(2-hydroxypropyl) methacylamide (HPMA) based copolymer containing polycationic, lipid and pka modulator moieties was developed for delivering the most potent siRNA and bipartite plasmid encoding two different shRNAs targeting AR and XIAP. HPMA based copolymer showed efficient cellular uptake in prostate cancer cells.

The findings reported in this work demonstrate the potential benefit of combination therapy targeting AR and XIAP pathways for treating prostate cancer using polymeric nanocarriers. Systematic chemical tailoring of polymers for improving drug loading as well as efforts made to improve micelle stability through crosslinking also generated insights which can be applied to other drugs and cancer types. Results reported in this study therefore offer a new way of addressing the time old problem of treating cancer. 


\section{TABLE OF CONTENTS}

CHAPTER 1. INTRODUCTION .1

Aim 1: To Establish a Novel Therapeutic Approach Simultaneously Targeting the Androgen Receptor (AR) and X-linked Inhibitor of Apoptosis Protein (XIAP) Pathways for Treating Prostate Cancer ....

Aim 2: To Develop a Series of Biodegradable and Biocompatible Copolymers for Enhanced Drug and Nucleic Acid Delivery ......................................................

Aim 3: To Explore the Utility of Polymeric Micelles as Drug Delivery Vehicles for

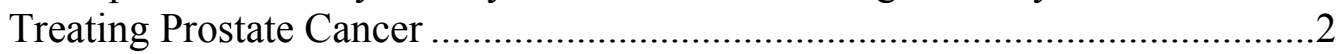

\section{CHAPTER 2. EXTRAVASATION OF POLYMERIC NANOMEDICINES ACROSS TUMOR VASCULATURE}

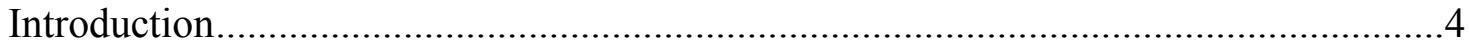

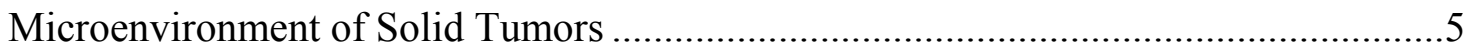

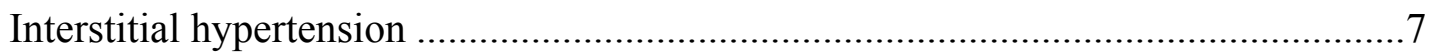

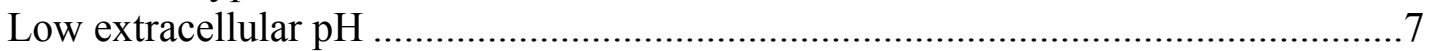

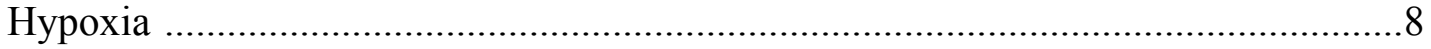

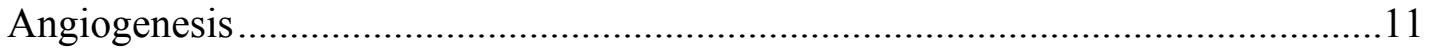

Tumor-stromal cell interaction..........................................................................

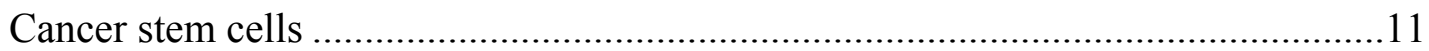

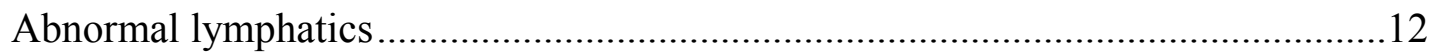

Anatomical Pathways of Macromolecular Extravasation..............................................12

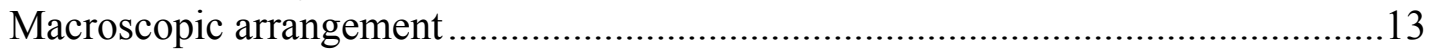

Tumor blood vessel wall structure .....................................................................13

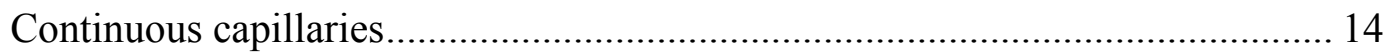

Fenestrated capillaries................................................................................. 14

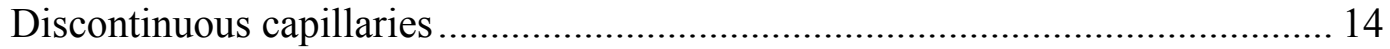

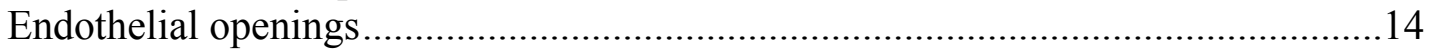

Vesiculo-vacuolar organelle mediated extravasation .................................................15

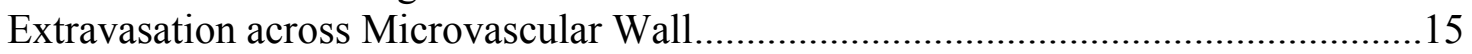

Barriers to Drug Delivery across Tumor Capillaries ....................................................17

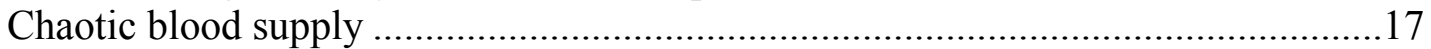

Poor permeability of tumor vasculature.................................................................. 17

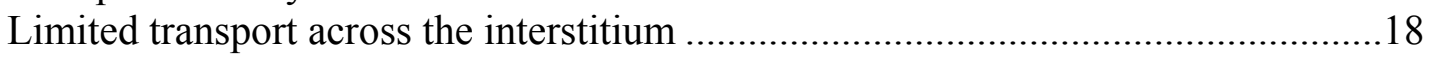

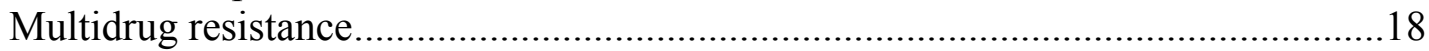

Mechanism responsible for multidrug resistance ………..................................... 18

Reversing multidrug resistance using polymeric nanomedicines .......................... 20

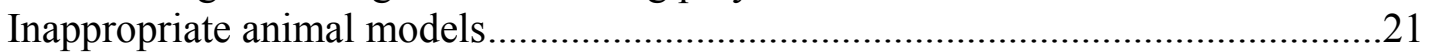

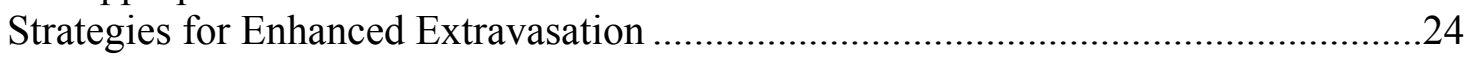

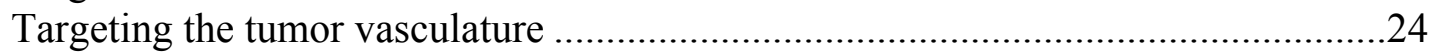

Lowering tumor interstitial hypertension.............................................................25

Increasing aqueous solubility of potent anti-cancer drugs........................................26

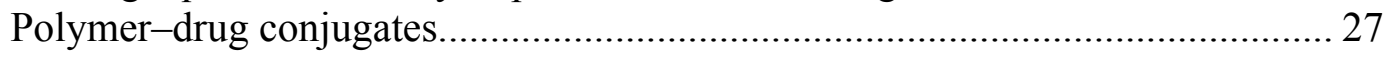

Polymeric micelles............................................................................................ 27 
Physics of drug encapsulation.......................................................................... 27

Compatibility between drug and core forming block ....................................... 28

Length of core forming block .................................................................... 30

Conclusions and Future Perspectives ……………….................................................30

\section{CHAPTER 3. MICELLAR DELIVERY OF BICALUTAMIDE AND EMBELIN} FOR TREATING PROSTATE CANCER ......................................................................33

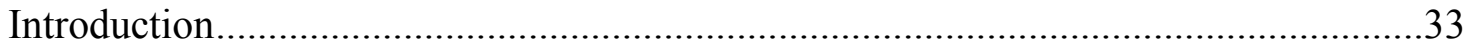

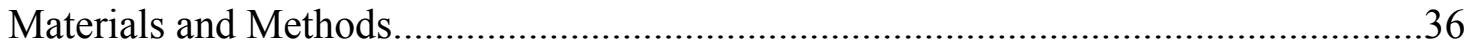

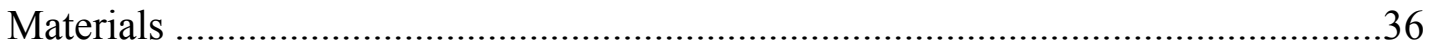

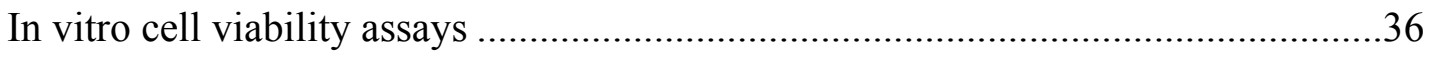

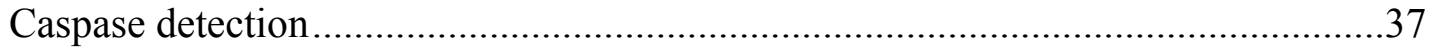

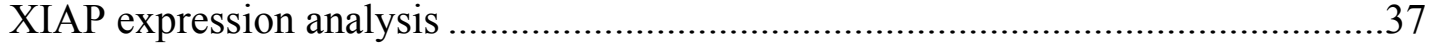

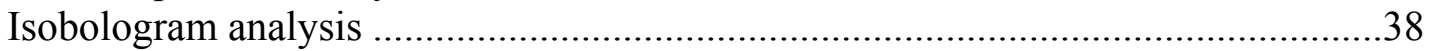

Fabrication of drug-loaded micelles ........................................................................

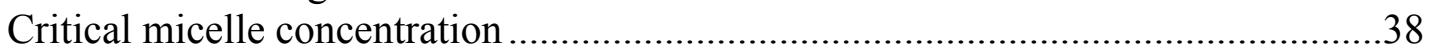

Drug loading density and encapsulation efficiency ………........................................39

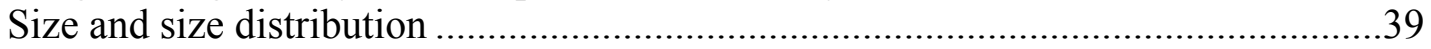

Solubility studies of drug-loaded micelles..................................................................39

In vivo efficacy assessment of bicalutamide-loaded micelles in xenografts ..............40

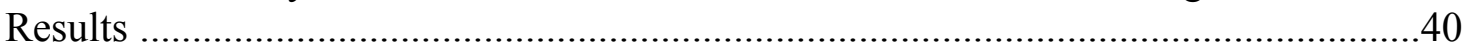

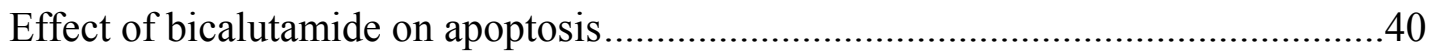

Effect of embelin on caspase activation and XIAP inhibiton ....................................40

Effect of bicalutamide and embelin on prostate cancer cell growth ............................43

Characterization of polymeric micelles .................................................................43

Drug encapsulation efficiency and micellar solubility ...............................................49

In vivo efficacy assessment of drug-loaded micelles in xenografts...........................49

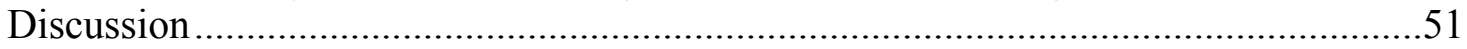

\section{CHAPTER 4. SELF-ASSEMBLING METHOXYPOLY(ETHYLENE GLYCOL)-B-POLY(CARBONATE-CO-L-LACTIDE) BLOCK COPOLYMERS FOR DRUG DELIVERY .........................................................56}

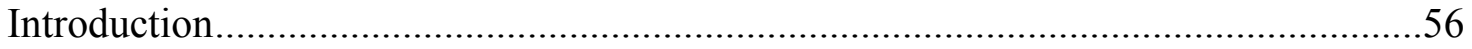

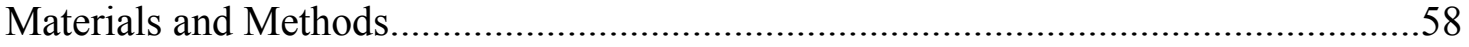

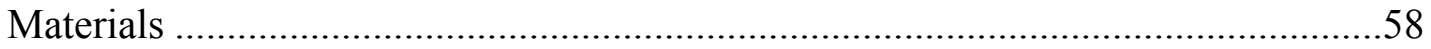

Computation of solubility and Flory-Huggins interaction parameters $\left(\chi_{\mathrm{FH}}\right)$ of bicalutamide with core of peg-b-plla/ peg-b-poly(carbonate-co-lactide)

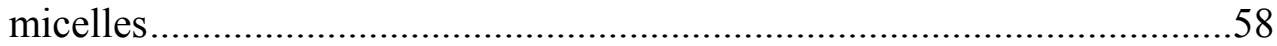

Synthesis of 5-Methyl-5-benzyloxycarbonyl-1,3-dioxane-2-one..............................58

Synthesis of peg-b-poly(carbonate-co-lactide) ……………....................................59

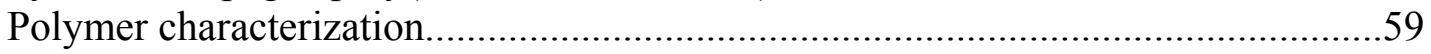

Nuclear magnetic resonance (NMR) .............................................................. 59

Gel permeation chromatography (GPC) ………………….............................. 59

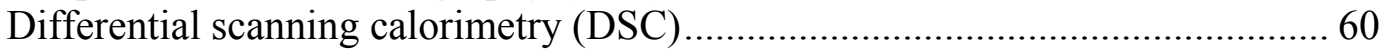

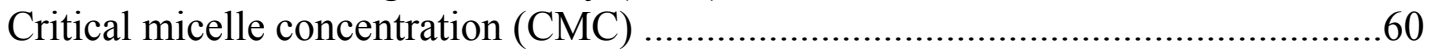

Preparation of bicalutamide-loaded micelles ..............................................................6 
Drug loading and encapsulation efficiency ............................................................61

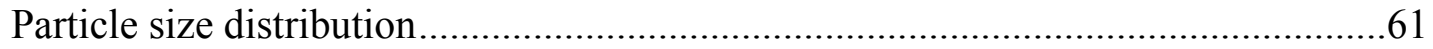

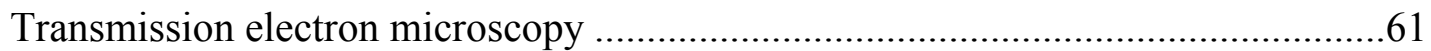

Bicalutamide release from micelles .....................................................................61

In vitro cytotoxicity of bicalutamide-loaded micelles .................................................62

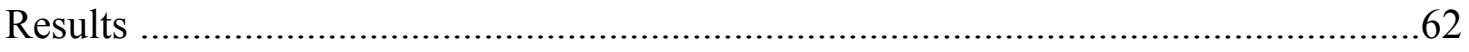

Design of diblock copolymer based on enhanced compatibility between bicalutamide and hydrophobic core ...

Synthesis and characterization of 5-Methyl-5-benzyloxycarbonyl-1,3-dioxane-2-

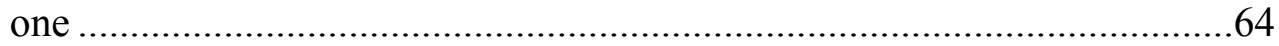

Synthesis and characterization of peg-b-poly(carbonate-co-lactide) copolymer.......64

Preparation and characterization of peg-b-poly(carbonate-co-lactide) copolymer

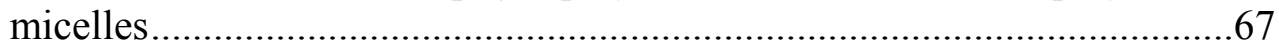

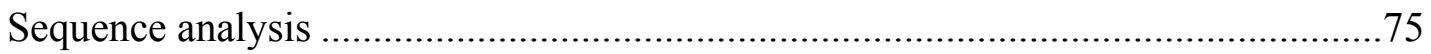

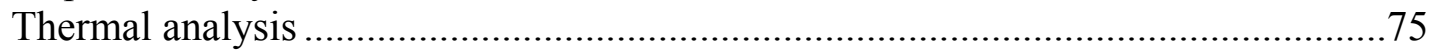

In vitro release studies of bicalutamide from micelles .............................................75

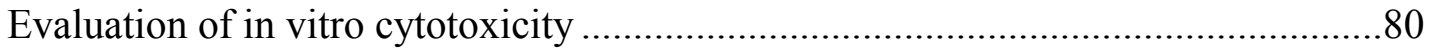

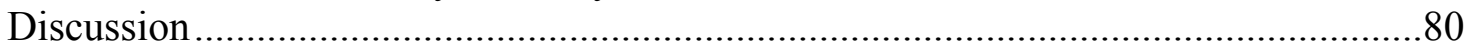

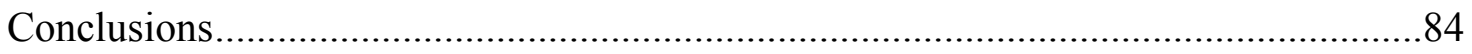

\section{CHAPTER 5. COMBINATION THERAPY OF ANTIANDROGEN AND XIAP} INHIBITOR FOR TREATING ADVANCED PROSTATE CANCER .......................86

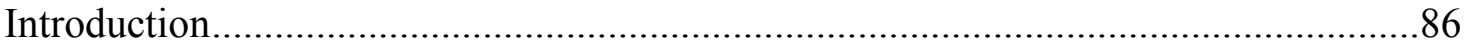

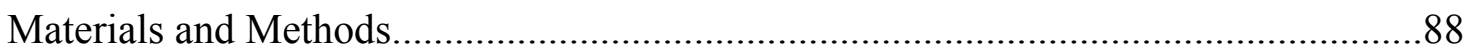

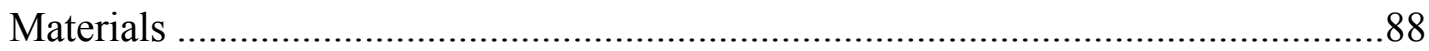

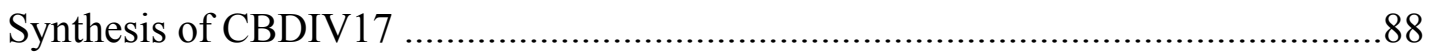

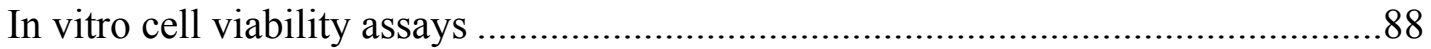

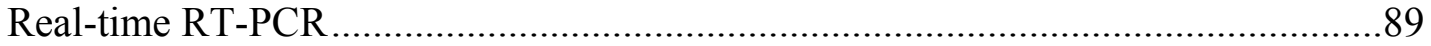

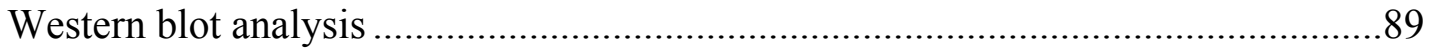

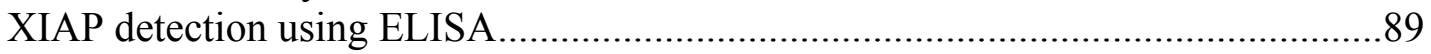

Apoptosis and cell cycle analysis .....................................................................89

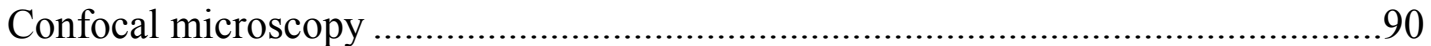

Preparation and characterization of micelles .......................................................99

In vivo efficacy of CBDIV17 and embelin-loaded micelles in mouse xenografts ....90

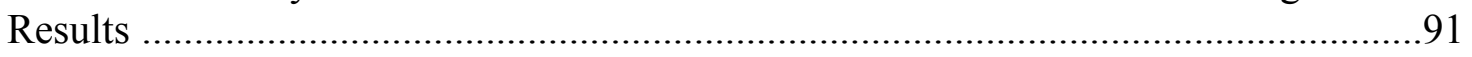

Synthesis and characterization of CBDIV17 …………........................................91

CBDIV17 and bicalutamide inhibit $\mathrm{LNCaP}$ and C4-2 cell growth ..........................91

CBDIV 17 and bicalutamide induce apoptosis and alter cell cycle of $\mathrm{LNCaP}$ and

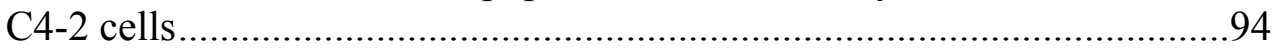

Embelin-6g is more potent than embelin in repressing XIAP expression ..................94

Embelin and its derivative downregulate AR expression and repress AR mediated activity ................................................................................94

Embelin blocks DHT-stimulated AR nuclear translocation and phosphorylation.....97

Embelin and its derivative inhibit prostate cancer cell growth ..................................97

Formulation of CBDIV17 and embelin-loaded PEG-b-p(CB-co-LA) micelles and their effect on prostate cancer cell growth and migration 
Effect of CBDIV17 and embelin combination on apoptosis 100

In vivo efficacy of CBDIV17 and embelin-loaded micelles in mouse xenografts ..103 Discussion. 103

\section{CHAPTER 6. LACTIC ACID AND CARBONATE BASED CROSSLINKED MICELLES FOR DRUG DELIVERY .................................................................108}

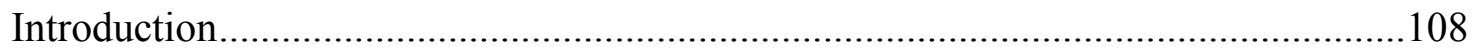

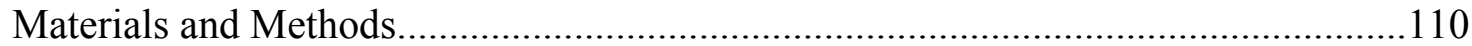

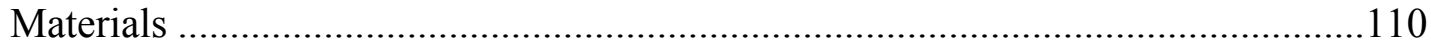

Synthesis of 5-Methyl-5-allyl-1,3-dioxane-2-one................................................110

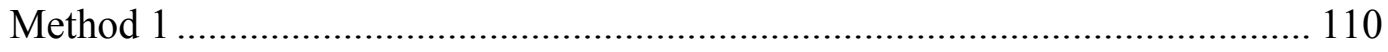

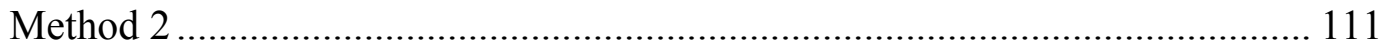

Synthesis of mPEG-b-P(lactide-co-carbonate-co-MAC).......................................111

Synthesis of mPEG-b-PMAC-b-P(lactide-co-carbonate) ……................................112

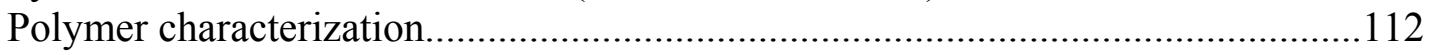

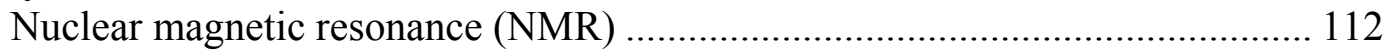

Gel permeation chromatography (GPC) …………....................................... 112

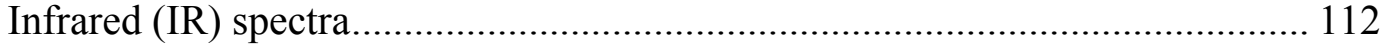

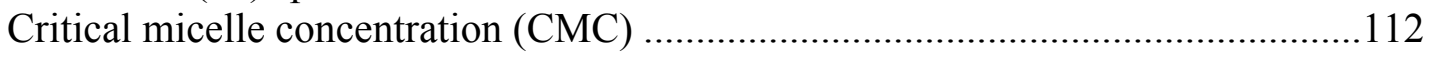

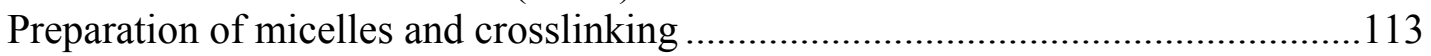

Drug loading and encapsulation efficiency.......................................................113

Particle size distribution and morphology ..........................................................113

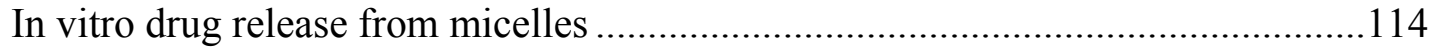

Assessment of in vitro micelle stability .............................................................114

In vitro cytotoxicity of bicalutamide-loaded micelles ............................................114

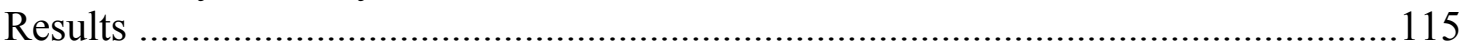

Synthesis and characterization of 5-Methyl-5-allyl-1,3-dioxane-2-one .................115

Synthesis and characterization of mPEG-b-PMAC-b-P(CB-co-LA) copolymer ....115

Synthesis and characterization of mPEG-b-P(CB-co-LA-co-MAC) copolymer.....120

Microstructure analysis and polymer toxicity.........................................................124

Effect of polymer composition and MAC location on CMC, particle size and

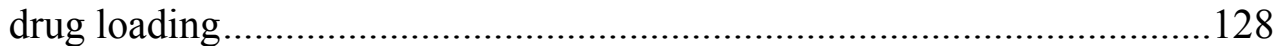

Effect of crosslinking on particle size, morphology and drug loading ....................131

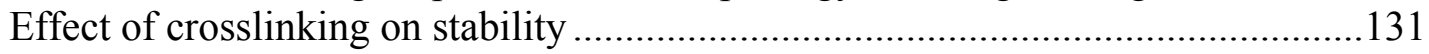

In vitro cytotoxicity of mPEG-b-PMAC-b-P(CB-co-LA) and mPEG-b-P(CB-co-

Discussion

LA-co-MAC) non-crosslinked (NCM) and crosslinked micelles (CM) ...136

Conclusions.

\section{CHAPTER 7. ANDROGEN RECEPTOR AND XIAP GENE SILENCING INHIBITS CELL PROLIFERATION AND INDUCES APOPTOSIS IN HORMONE REFRACTORY PROSTATE CANCER}

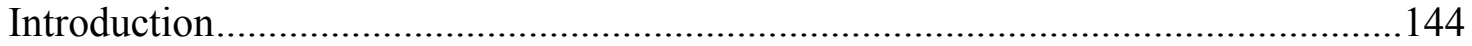

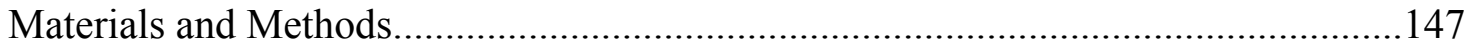

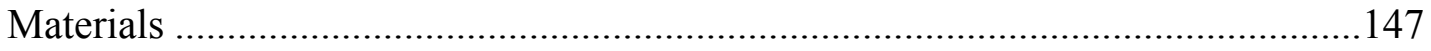

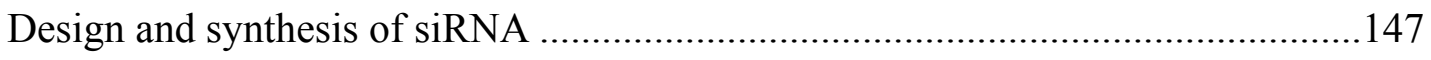


Synthesis of HPMA-g-APMA-g-MM-g-HM copolymers.......................................147

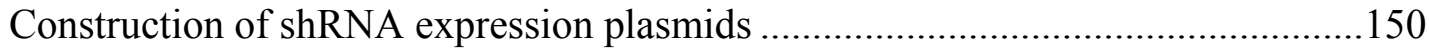

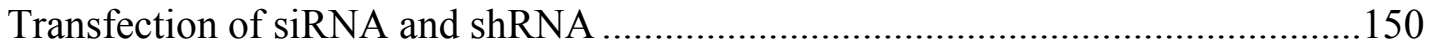

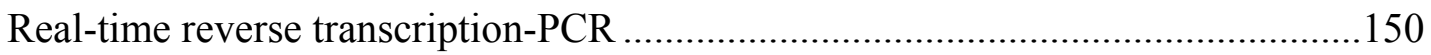

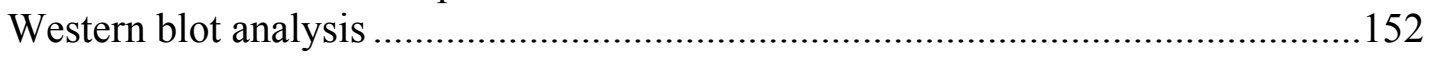

XIAP concentration detection using ELISA ..........................................................152

siRNA uptake and cellular distribution study by flow cytometry and confocal

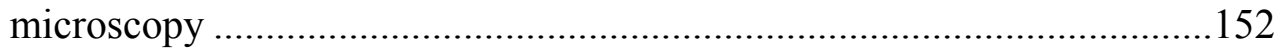

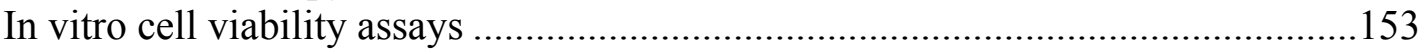

Apoptosis and cell cycle analysis ......................................................................153

In vivo efficacy assessment of siRNA/shRNA polyplex in xenografts ....................153

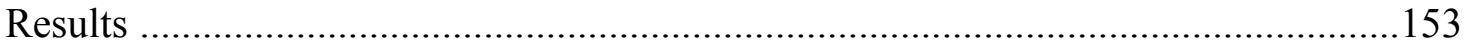

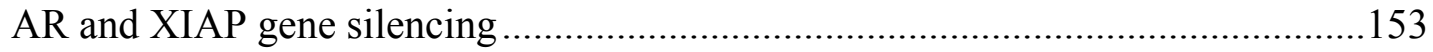

Synthesis and characterization of HPMA-g-APMA-g-MM-g-HM copolymers .....154

Effect of HPMA-g-APMA-g-MM-g-HM copolymers on nucleic acid condensation and polyplex size...............................................................157

Effect of charge ratio on cellular uptake of polymer/siRNA complexes..................157

Effect of $\mathrm{HPMA}_{32}-\mathrm{g}-\mathrm{APMA}_{3}-\mathrm{g}-\mathrm{MM}_{22}-\mathrm{g}-\mathrm{HM}_{43}$ and Lipofectamine 2000 on AR and XIAP gene silencing .......................................................................157

$\mathrm{AR}$ and XIAP gene silencing induces apoptosis and inhibits $\mathrm{C} 4-2$ cell

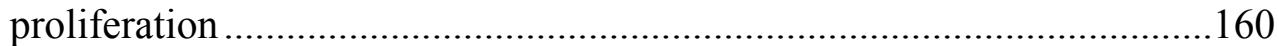

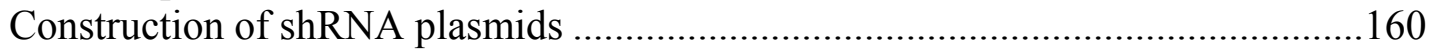

Effect of $\mathrm{HPMA}_{32}$-g-APMA $-\mathrm{g}-\mathrm{MM}_{22}$-g- $\mathrm{HM}_{43}$ copolymer on plasmid transfection efficiency and condensation .................................................163

Effect of shRNA sequence on AR gene silencing .................................................163

Effect of shRNA expression on AR and XIAP gene silencing ................................163

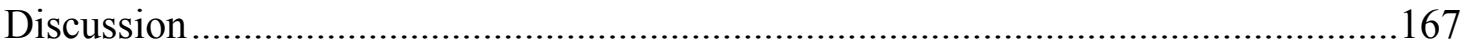

CHAPTER 8. CONCLUSIONS AND FUTURE RESEARCH ..................................169

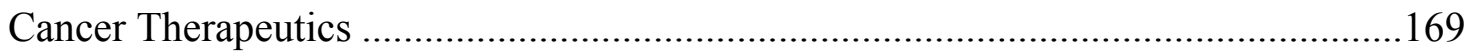

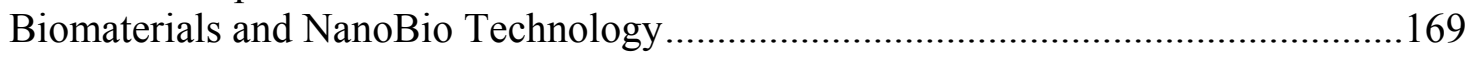

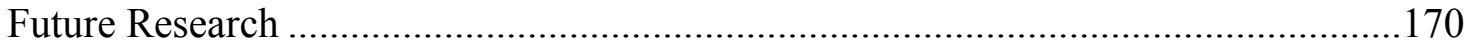

LIST OF REFERENCES...............................................................................................172

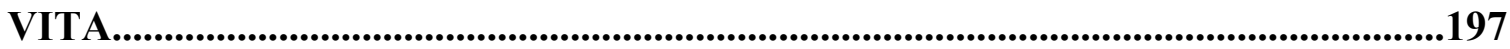




\section{LIST OF TABLES}

Table 2-1. Multidrug resistance mechanisms.

Table 3-1. Synergistic antiproliferative activity of bicalutamide $(\mathrm{d} 1=10 \mu \mathrm{M})$ and embelin in human prostate cancer cells.

Table 3-2. Synergistic antiproliferative activity of bicalutamide $(\mathrm{d} 1=25 \mu \mathrm{M})$ and embelin in human prostate.

Table 3-3. Synergistic antiproliferative activity of bicalutamide $(\mathrm{d} 1=50 \mu \mathrm{M})$ and embelin in human prostate.

Table 4-1. Calculated Hansen Solubility Parameters of bicalutamide, PLLA and $\mathrm{P}(\mathrm{CB}-\mathrm{co}-\mathrm{LA})$.

Table 4-2. $\quad$ Effect of carbonate content on the molecular weight as determined by ${ }^{1} \mathrm{H}$ $\mathrm{NMR}$ and $\mathrm{GPC}$ and $\mathrm{CMC}$ of micelles at $25^{\circ} \mathrm{C}$.

Table 4-3. Effect of polymer composition on drug loading and particle size of bicalutamide-loaded micelles.

Table 6-1. Characteristics of lactic acid and carbonate-based copolymers.

Table 6-2. Effect of polymer composition and MAC location on particle size and drug loading in noncrosslinked micelles.

Table 6-3. Effect of polymer composition and MAC location on particle size and drug loading in crosslinked micelles.

Table 7-1. Small interfering RNA (siRNA) against human androgen receptor and $\mathrm{X}$-linked inhibitor of apoptosis.

Table 7-2. Characterization of HPMA-g-APMA-g-MM-g-HM copolymers.

Table 7-3. shRNA sequences targeting specific regions of androgen receptor (AR) and XIAP genes. 


\section{LIST OF FIGURES}

Figure 2-1. Schematic portraying complex microenvironment of tumors. ....................6

Figure 2-2. Schematic depicting $\mathrm{pH}$ triggered cleavage and drug release of poly (ethylene glycol)-poly(aspartate-hydrazone-drug) delivery system in acidic endosomal compartment.

Figure 2-3. Crucial factors contributing to hypoxia in tumors.

Figure 2-4. Schematic representation of convective and diffusive transport of polymeric nanomedicines across microvascular wall and the effect of pore cut off size.

Figure 2-5. Chemical structure and anticancer effect of SMART-100.

Figure 2-6. Anticancer effect of antiandrogen and XIAP inhibitor based combination therapy.

Figure 2-7. Effect of carbonate content on key poly (ethylene glycol)-b-poly (carbonate-co-lactide) micelle properties.

Figure 2-8. Influence of lipid substituent group on polymer architecture and embelin loading.

Figure 3-1. Therapeutic strategy for bicalutamide and embelin based combination therapy.

Figure 3-2. Effect of bicalutamide on the activation of caspases in LNCaP cells.

Figure 3-3. Effect of Embelin on caspase activation and XIAP inhibition in LNCaP and C4-2 prostate cancer cells.

Figure 3-4. Effect of drug treatment on cell viability in prostate cancer cells (C4-2 and $\mathrm{LNCaP}$ ).

Figure 3-5. Dose-response curves for bicalutamide and embelin combination in human prostate cancer cells.

Figure 3-6. Effect of theoretical loading on drug loading density and solubility.

Figure 3-7. Effect of bicalutamide and embelin-loaded micelles on growth of tumors derived from $\mathrm{LNCaP}$ prostate cancer cells in nude mice.

Figure 4-1. Synthesis method of PEG-b-P(CB-co-LA) copolymers. 
Figure 4-2. ${ }^{1} \mathrm{H}$ NMR spectra in $\mathrm{CDCl}_{3}$ of (A) 5-Methyl-5-benzyloxycarbonyl-1,3dioxane-2-one (carbonate monomer) and (B) $\mathrm{PEG}_{114}-\mathrm{b}-\mathrm{P}\left(\mathrm{CB}_{9}-\mathrm{co}-\mathrm{LA}_{33}\right)$ copolymer.

Figure 4-3. Gel permeation chromatograms of PEG-b-PLLA and $\mathrm{PEG}_{114}-\mathrm{b}-\mathrm{P}\left(\mathrm{CB}_{9}\right.$ co-LA $\mathrm{L}_{33}$ ) copolymers.

Figure 4-4. Micelle size distribution and surface morphology.

Figure 4-5. Plots of intensity ratio $\mathrm{I}_{338} / \mathrm{I}_{333}$ from pyrene fluorescence emission spectra versus log concentration (g/L) of PEG-b-PLLA and PEG-b$\mathrm{P}(\mathrm{CB}-\mathrm{co}-\mathrm{LA})$ copolymers.

Figure 4-6. ${ }^{1} \mathrm{H}$ NMR spectra of bicalutamide-loaded $\mathrm{PEG}_{114}-\mathrm{b}-\mathrm{P}\left(\mathrm{CB}_{8}-\mathrm{co}-\mathrm{LA}_{24}\right)$ micelles.

Figure 4-7. ${ }^{13} \mathrm{C}$ NMR spectra of $\mathrm{PEG}_{114}-\mathrm{b}-\mathrm{P}\left(\mathrm{CB}_{8}\right.$-co-LA $\left.\mathrm{L}_{24}\right)$ copolymer. . .76

Figure 4-8. ${ }^{13} \mathrm{C}$ NMR spectra comparative plot for 10,20 and $40 \mathrm{~mol} \%$ carbonate content demonstrating decrease in LLL sequence and increase in CCC sequence with increasing carbonate content.

Figure 4-9. DSC thermograms of PEG-b-PLLA and PEG-b-P(CB-co-LA) copolymers

Figure 4-10. Effect of carbonate content on bicalutamide release from PEG-b-PLLA and PEG-b-P(CB-co-LA) micelles.

Figure 4-11. Effect of free and PEG-b-P(CB-co-LA) micellar formulation of bicalutamide on inhibition of LNCaP cell proliferation $48 \mathrm{~h}$ post treatment

Figure 5-1. Synthesis and characterization of (S)-N-(4-cyano-3(trifluoromethyl)phenyl)-3-((4-cyanophenyl)(methyl)amino)-2-hydroxy2-methylpropanamide) (CBDIV17).

Figure 5-2. Anticancer effect of antiandrogen on prostate cancer cells.

Figure 5-3. Effect of bicalutamide and CBDIV17 on cell proliferation in the presence of androgen.

Figure 5-4. Effect of bicalutamide and CBDIV17 on apoptosis and cell cycle of LNCaP and C4-2 cells. .95

Figure 5-5. Embelin and embelin-6g suppress XIAP expression. .96

Figure 5-6. Effect of embelin and embelin-6g on AR and PSA expression. .98 
Figure 5-7. Effect of embelin and embelin-6g on prostate cancer cell proliferation and cyclin D1, expression.

Figure 5-8. Effect of CBDIV17 and embelin combination on prostate cancer cell growth and migration.

Figure 5-9. Effect of embelin and CBDIV17 combination on cell cycle and apoptosis

Figure 5-10. Effect of CBDIV17 and embelin-loaded micelles on growth of tumors derived from $\mathrm{C} 4-2$ prostate cancer cells in nude mice.

Figure 6-1. Synthesis and characterization of 5-methyl-5-allyl-1,3-dioxane-2-one (MAC).

Figure 6-2. FT-IR spectrum of 5-methyl-5-allyl-1,3-dioxane-2-one (MAC).

Figure 6-3. Synthesis method for preparation of interface-crosslinked micelles.

Figure 6-4. Methoxy poly (ethylene glycol) and MAC copolymerization in $\mathrm{CH} 2 \mathrm{Cl} 2$ at room temperature.

Figure 6-5. $\quad{ }^{1} \mathrm{H}$ NMR spectra for lactic acid and carbonate-based copolymer for crosslinked micelles.

Figure 6-6. Synthesis method for preparation of lactic acid and carbonate-based crosslinked micelles.

Figure 6-7. ${ }^{13} \mathrm{C}$ NMR spectra for lactic acid and carbonate-based copolymer for crosslinked micelles.

Figure 6-8. ${ }^{13} \mathrm{C}$ NMR spectra comparative plot for mPEG- $b$-PMAC, mPEG- $b$ PMAC- $b$-P(CB-co-LA), mPEG- $b$-P(CB- $c o$-LA- $c o$-MAC) and mPEG- $b$ $\mathrm{P}(\mathrm{CB}-c o-\mathrm{LA})$ demonstrating differences in monomer arrangement.

Figure 6-9. Cytotoxicity of mPEG- $b$-PMAC- $b$-P(CB-co-LA) and mPEG- $b$-P(CBco-LA-co-MAC) copolymers in prostate cancer cells.

Figure 6-10. ${ }^{1} \mathrm{H}$ NMR spectra for methoxy poly (ethylene glycol)-b-poly (5-methyl-5allyl-1,3-dioxane-2-one-co-carbonate-co-lactide) [mPEG- $b$-P(MAC-coCB-co-LA)] copolymer in $\mathrm{CDCl}_{3}$.

Figure 6-11. FTIR spectra for lactic acid and carbonate-based copolymer.

Figure 6-12. TEM images of (A) non-crosslinked and (B) crosslinked micelles

Figure 6-13. Stability of mPEG-b-PMAC-b-P(CB-co-LA) non-crosslinked (NCM) and crosslinked micelles (CM). 
Figure 6-14. Anticancer effect of mPEG- $b$-PMAC- $b$-P(CB-co-LA) and mPeg- $b$ $\mathrm{P}(\mathrm{CB}-$ so-LA-co-MAC) non-crosslinked (NCM) and crosslinked micelles $(\mathrm{CM})$.

Figure 6-15. Effect of bicalutamide-loaded mPEG- $b$-PMAC- $b-\mathrm{P}(\mathrm{CB}-c o-\mathrm{LA})$ and mPEG- $b$-P(CB-co-LA-co-MAC) non-crosslinked (NCM) and crosslinked micelles $(\mathrm{CM})(50 \mu \mathrm{M}$ of drug) on secreted prostate specific antigen (PSA) following $24 \mathrm{~h}$ incubation

Figure 7-1. Schematic diagram showing the combined effect of androgen receptor and XIAP gene silencing on apoptosis and tumor regression.

Figure 7-2. Effect of siRNA sequence on AR and XIAP gene silencing after complex formation with Lipofectamine 2000.

Figure 7-3. Synthesis of HPMA-g-APMA-g-MM-g-HM copolymers

Figure 7-4. Effect of charge (N/P) ratio on siRNA condensation, polyplex size and cellular uptake.

Figure 7-5. Comparison of $\mathrm{HPMA}_{32}$-g-APMA 3 -g-MM $\mathrm{MM}_{22}$-g-HM 43 and lipofectamine 2000 on AR and XIAP gene silencing.

Figure 7-6. Effect of AR and XIAP gene silencing on apoptosis and proliferation in C4-2 cells.

Figure 7-7. Schematic representation of shRNA expression vectors encoding single shRNAs targeting AR or XIAP or bipartite vector encoding shRNA simultaneously targeting AR and XIAP driven by two 7SK promoters....162

Figure 7-8. Effect of $\mathrm{HPMA}_{32}-\mathrm{g}-\mathrm{APMA}_{3}-\mathrm{g}-\mathrm{MM}_{22}-\mathrm{g}-\mathrm{HM}_{43}$ on transfection efficiency and condensation.

Figure 7-9. Effect of shRNA sequence and time on androgen receptor (AR) gene silencing.

Figure 7-10. Effect of shRNA expression on AR and XIAP gene silencing. 166 


\section{LIST OF ABBREVIATIONS}

$\begin{array}{ll}\chi_{\text {FH }} & \text { Flory-Huggins interaction parameter } \\ \text { ABC } & \text { ATP-binding cassette } \\ \text { AR } & \text { Androgen receptor } \\ \text { BBB } & \text { Blood brain barrier } \\ \text { BCRP } & \text { Breast cancer resistance protein } \\ \text { BFGF } & \text { Basic fibroblast growth factor } \\ \text { BIR } & \text { Baculovirus inhibitor of apoptosis protein repeat } \\ \text { CB } & \text { 5-methyl-5-benzyloxycarbonyl-1,3-dioxane-2-one } \\ \text { CBDIV17 } & \text { (S)-N-(4-cyano-3-trifluoromethyl) phenyl)-3-((4- } \\ & \text { cyanophenyl)(methyl)amino)-2-hydroxy-2-methylpropanamide } \\ \text { CM } & \text { Crosslinked micelles } \\ \text { CSCs } & \text { Cancer stem cells } \\ \text { DHT } & \text { 5a-Dihydrotestosterone } \\ \text { DLS } & \text { Dynamic light scattering } \\ \text { DMSO } & \text { Dimethyl sulfoxide } \\ \text { DOX } & \text { Doxorubicin } \\ \text { EMBELIN-6G } & \text { 2,5-dihydroxy-3-(2-[4-(2-m-tolyl-ethyl)-phenyl]-ethyl)-[1,4] } \\ & \text { benzoquinone } \\ \text { EPR } & \text { Enhanced permeability and retention } \\ \text { GSH } & \text { Gluthathione } \\ \text { GST } & \text { Gluthathione S-transferase } \\ \text { HIF-1 } & \text { Hypoxia inducible factor-1 } \\ \text { HRPC } & \text { Hormone refractory prostate cancer } \\ \text { IAP } & \text { Inhibitor of apoptosis } \\ \text { IL-8 } & \text { Interleukin-8 } \\ \text { Lp } & \text { Hydraulic conductivity } \\ \text { MAC } & \text { 5-Methyl-5-allyl-1,3-dioxane-2-one } \\ \text { MDR } & \text { Multidrug resistance } \\ \text { MPS } & \text { Mononuclear phagocyte system } \\ \text { MRP } & \text { Multidrug resistance-associated proteins } \\ \text { MTC-OH } & \text { 5-methyl-2-oxo-1,3-dioxane-5-carboxylic acid } \\ \text { NCM } & \text { Non-crosslinked micelles } \\ \text { P } & \text { Vascular permeability } \\ \text { PDGF } & \text { Platelet-derived growth factor } \\ \text { PEG } & \text { Poly(ethylene glycol) } \\ \text { PEG-b-(CB-co-LA) } & \text { Poly (ethylene glycol)-b-poly (carbonate-co-lactide) } \\ \text { PEG-b-PAA } & \text { Poly (ethylene glycol)-b-poly (aspartic acid) } \\ \text { PEG-b-PCD } & \text { Poly(ethylene glycol)-block-poly(2-methyl-2-carboxyl-propylene } \\ & \text { carbonate-graft-dodecanol) } \\ \text { PEG-b-PCL } & \text { Poly (ethylene glycol)-b-poly(caprolactone) } \\ \text { PEG-b-PDLLA } & \text { Poly (ethylene glycol)-b-poly(D,L-lactide) } \\ \text { PEG-b-PLGA } & \text { Poly (ethylene glycol)-b-poly(lactide-co-glycolic acid) } \\ \text { PEG-b-PLLA } & \text { Poly (ethylene glycol)-b-poly (L-Lactide) } \\ & \end{array}$




$\begin{array}{ll}\text { PEO } & \text { Poly(ethylene oxide) } \\ \text { P-gp } & \text { P-glycoprotein } \\ \text { PLLA } & \text { Poly(l-lactic acid) } \\ \text { PLN } & \text { Polymer-lipid hybrid nanoparticles } \\ \text { PolyHis } & \text { Poly(l-histidine) } \\ \text { PSA } & \text { Prostate specific antigen } \\ \text { RES } & \text { Reticuloendothelial system } \\ \text { SEM } & \text { Scanning electron microscopy } \\ \text { shRNA } & \text { Short hairpin RNA } \\ \text { SiRNA } & \text { Small interfering RNA } \\ \text { SMART-100 } & \text { Methoxybenzoyl-ary-thiaozole } \\ \text { TGF- } \beta & \text { Transforming growth factor- } \beta \\ \text { VEGF } & \text { Vascular endothelial growth factor } \\ \text { VPF } & \text { Vascular permeability factor } \\ \text { VVOs } & \text { Vesiculovacuolar organelles } \\ \text { XIAP } & \text { X-chromosome-linked inhibitor of apoptosis }\end{array}$




\section{CHAPTER 1. INTRODUCTION}

Prostate cancer is the most prevalent malignancy affecting men in the United States and the second most lethal cancer. Approximately, 241,740 new cases are expected to be diagnosed and 28,170 men are projected to die from the disease in the United States in 2012 [1]. Since most prostate cancer patients present with the disease already beyond the confines of the prostate, radical prostatectomy which is potentially curative is not possible. Instead, nonsurgical approaches such as androgen ablation remain the preferred therapy for patients with advanced prostate cancer. Although, androgen deprivation therapy is initially highly effective, approximately $70-80 \%$ of patients eventually relapse. The fatal nature of prostate cancer is partly due to chemoresistance and the inability to selectively deliver potent anticancer agents to cancer cells.

Therefore, the focus of this research was to develop and investigate a novel holistic approach for treating chemoresistant prostate cancer tumors with the goal that insights obtained can be translated to treating other chemoresistant tumors. Consequently, three highly focused aims hinging on fundamental cancer biology, creation of biomaterials and application of nanobiotechnology for improving cancer therapy were stipulated and addressed. The aims specifically examined and their attendant objectives are as follows:

\section{Aim 1: To Establish a Novel Therapeutic Approach Simultaneously Targeting the Androgen Receptor (AR) and X-linked Inhibitor of Apoptosis Protein (XIAP) Pathways for Treating Prostate Cancer}

Objectives for Aim 1 are given below.

(i) Examine growth inhibitory effect and apoptotic cell death following treatment with bicalutamide (AR antagonist) and embelin (XIAP inhibitor).

(ii) Determine whether XIAP inhibitor (embelin and embelin-6g) exhibit synergy with second generation antiandrogen in inhibiting tumor growth in vitro and in vivo.

(iii) Elucidate molecular mechanism of XIAP inhibitor on prostate cancer

(iv) Utilize siRNA targeting AR and XIAP genes alone and in combinations for treating prostate cancer.

(v) Construct a bipartite plasmid expressing shRNAs simultaneously targeting $\mathrm{AR}$ and XIAP genes for combination therapy for treating prostate cancer. 


\section{Aim 2: To Develop a Series of Biodegradable and Biocompatible Copolymers for Enhanced Drug and Nucleic Acid Delivery}

Objectives for Aim 2 are given below.

(i) Design and develop tailor made biodegradable copolymers for antiandrogen delivery.

(ii) Synthesize, characterize and evaluate lactic acid and carbonate-based copolymers and characterize drug loading, release profiles, and micellar stability.

(iii) Synthesize, characterize and evaluate lactic acid and carbonate-based copolymers for core or core/corona interface crosslinked micelles for enhanced stability of micelles.

(iv) Synthesize, characterize and asssess N-(2-hydroxypropyl) methacrylamide (HPMA) based copolymer composed of polycationic, lipid and pka modulator moieties for efficient delivery of nucleic acids.

\section{Aim 3: To Explore the Utility of Polymeric Micelles as Drug Delivery Vehicles for Treating Prostate Cancer}

Objectives for Aim 3 are given below.

(i) Assess effect of polymeric micelles on drug solubilization and efficacy.

(ii) Determine whether XIAP inhibitor and antiandrogen formulated polymeric micelle exhibits synergy in inhibiting prostate tumor growth in vitro and in vivo.

The remainder of the dissertation is organized in the following manner. Review of the pertinent literature regarding tumor microenvironment, intrinsic barriers to therapy and the role of polymeric nanomedicines in overcoming these barriers are presented in Chapter 2. Results showing the potential of bicalutamide and embelin combination therapy for treating prostate cancer in vitro and in vivo are presented in Chapter 3. Chapter 4 covers the design, synthesis, characterization and in vitro evaluation of polyethylene glycol-b-poly (carbonate-co-lactide) [PEG-b-P(CB-co-LA)] copolymers for improved bicalutamide loading and micelle thermodynamic stability. Findings demonstrating superior anticancer activity of a newly synthesized antiandrogen ((S)-N(4-cyano-3-(trifluoromethyl) phenyl)-3-((4-cyanophenyl)(methyl)amino)-2-hydroxy-2methylpropanamide) (CBDIV17)) and supra additive effects observed in combination with XIAP inhibitor for treating prostate cancer is reported in Chapter 5. A possible additional mechanism of embelin as a modulator of the AR is also presented in Chapter 5. Chapter 6 describes the design, synthesis, characterization and in vitro assessment of lactic acid and carbonate-based copolymers for core and core-corona interface crosslinked micelles demonstrating improved micelle stability. The results of simultaneous silencing of AR and XIAP genes using RNA interference and their effect on prostate cancer cell growth and apoptosis is given in Chapter 7. Synthesis and 
evaluation of N-(2-hydroxypropyl) methacrylamide (HPMA) based copolymer possessing polycationic, lipid and pka modulator moieties for nucleic acid delivery is also addressed in Chapter 7. Finally, the conclusions of this work are presented in Chapter 8. 


\section{CHAPTER 2. EXTRAVASATION OF POLYMERIC NANOMEDICINES ACROSS TUMOR VASCULATURE*}

\section{Introduction}

Tumors possess poorly differentiated, highly chaotic arrangement of vessels which have unsealed endothelial cell-cell junctions and discontinuous basement membrane. The "leaky" nature of tumor vessels is accompanied with functionally impaired tumor vasculature resulting in high permeability of macromolecules in tumors compared to normal tissue. Tumors also have functionally defective lymphatic vessels. The decreased lymphatic drainage associated with tumors results in retention of permeant macromolecules in tumors since the fluid that leaves the bloodstream inside the tumor can only escape from the tumor either by feeding back into postcapillary venules or by passage through the tumor interstitium.

Delivery of anticancer agents to solid tumors remains a significant challenge. First, anticancer drugs have low tumor to normal cell selectivity making them potentially toxic to both normal and tumor cells. Moreover, normal tissues possess low intrinsic tolerance compared to tumors severely limiting the dose required for tumor regression [2, 3]. Second, most anticancer agents are highly lipophilic, requiring the use of solubilizing agents or surfactants so that they can be used via systemic administration. Paclitaxel, for example, is a potent anticancer drug with aqueous solubility of only $\sim 1 \mu \mathrm{g} / \mathrm{mL}$ [4]. Docetaxel, its more water soluble derivative also has a low aqueous solubility of $\sim 6$ $7 \mu \mathrm{g} / \mathrm{mL}$ [5]. Hence, solubilizing agents such as dimethyl sulfoxide (DMSO), Cremophor ${ }^{\circledR}$ EL or Tween 80 are typically used to bring these drugs into true solution for systemic administration. However, such agents are harmful to the liver and kidneys [6], cause dose-dependent hemolysis and lead to neurotoxicity [7] thus hindering their medical utility for intravenous applications.

Biodegradable polymeric or lipid carriers are attractive candidates for anticancer drug delivery since they lead to high therapeutic concentrations of anticancer agents to tumors, with minimal systemic exposure. Examples include nanoparticles, liposomes [8], micelles [9-11], and polymer-drug conjugates [12]. These nanosized delivery platforms are capable of improving drug solubility and take advantage of the unique pathophysiology of tumor vasculature to ferry hydrophobic drugs with minimal adverse effects upon systemic administration. Typically this occurs by the enhanced permeability and retention (EPR) effect where macromolecular systems preferentially accumulate in tumors when administered systemically [13-15].

Successful treatment of cancer utilizing polymeric nanomedicines requires that once blood borne, they reach the site of action in optimal quantities. To achieve this, they

*Danquah MK, Zhang XA, Mahato RI. Extravasation of polymeric nanomedicines across tumor vasculature. Adv Drug Deliv Rev. 2011;63:623-39. 
must arrive at the tumor through blood vessels, be transported across vascular walls (extravasation) and then move through the interstitium. Jain and others have shown tremendous heterogeneity in vessel permeability within tumors and between tumor types. Aberrant extravasation has been implicated in the heterogeneous distribution of drugs inside the tumors accounting for their moderate therapeutic effect in complete tumor eradication. Hence, overcoming this obstacle of nonuniform extravasation is crucial to improving the drug delivery potential of polymeric nanomedicines. This challenge demands an understanding of the mechanisms governing vascular permeability and the spatial and temporal regulation of transport pathways in tumors [16]. Insight from such understanding can be utilized in developing the next generation of drug delivery polymeric nanomedicines possessing tunable properties that facilitate their extravasation across tumor vasculature and discovering superior approaches for modulating tumor vasculature.

In this review, we summarize current knowledge on the distinct tumor microenvironment and blood vessel structure, talk about barriers to drug delivery to tumors, mechanisms of barrier formation and finally appraise modalities for overcoming these barriers.

\section{Microenvironment of Solid Tumors}

To develop potent therapeutic approaches for site-specific delivery of drugs to tumors using polymeric nanomedicines, it is imperative to understand the microenvironment of the tumor. Solid tumors are organ-like entities arising from stem cell populations and consist of cancer cells, non-transformed stromal cells, blood vessels and the interstitium [17]. Cancer and stromal cells comprise the major portion of solid tumors accounting for greater than $50 \%$ of tumor volume. Blood vessels nourish both stromal and cancer cells in tumors and constitutes up to $10 \%$ of tumor volume, while the interstitium forms the remainder of the tumor and provides the nutritional and structural framework for it to grow.

While the normal cellular microenvironment can inhibit malignant cell growth, the tumor microenvironment supports cell proliferation. Tumors shape their microenvironment and support the tumor and non-malignant cells. Tumor microenvironment greatly influences its development and generates barriers (Figure 2-1) that prevent therapeutic agents from accessing and killing cancerous cells in the tumor, thereby limiting the efficacy of current chemotherapy [18]. Below, we discuss interstitial hypertension, low extracellular $\mathrm{pH}$, hypoxia, angiogenesis, tumor-stromal cell interaction, cancer stem cells, and abnormal lymphatics. These key microenvironmental features of solid tumors greatly impact the extravasation of polymeric nanomedicines. 


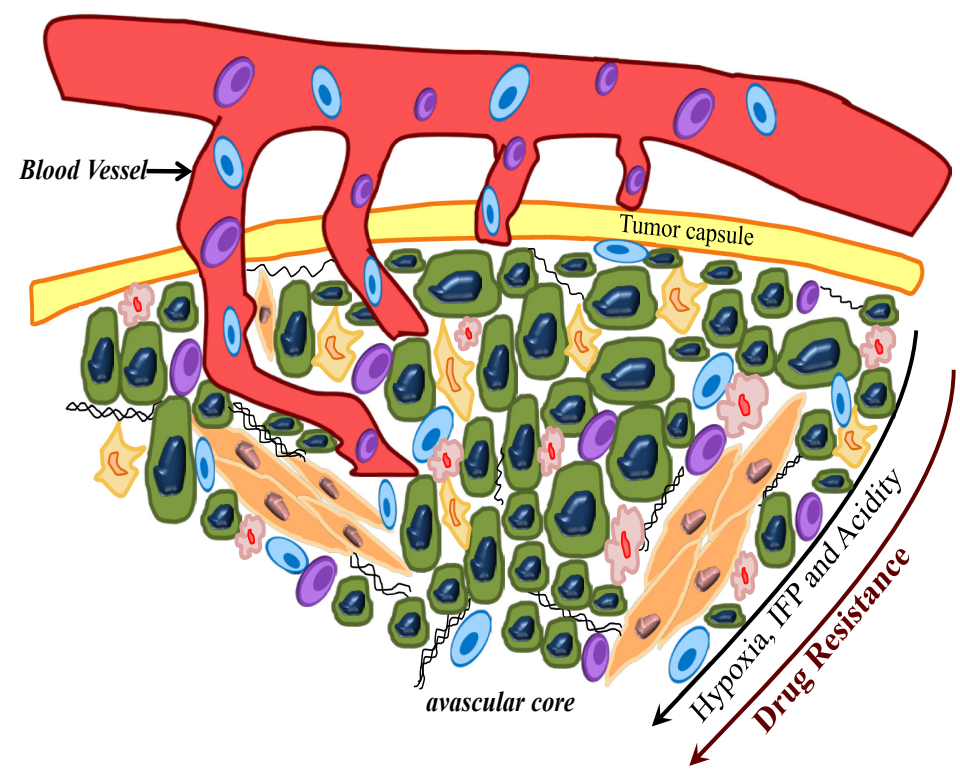
Cancer cell
Lymphocyte
Monocyte
Fibroblast
Stem cell
$55^{\circ f^{\infty} \text { Extracellular matrix }}$
Macrophage

\section{Figure 2-1. Schematic portraying complex microenvironment of tumors.}

Cancer cells are surrounded by cancer stem cells and embedded within the stroma composed of lymphocytes,monocytes, fibroblasts, macrophages and extracellular matrix. Tumor-stroma interaction is bidirectional and may lead to tumor rejection or facilitate tumor growth. Key tumor microenvironmental features: hypoxia, interstitial fluid pressure (IFP) and acidity increase with distance from blood vessels resulting in increasing drug resistance from the tumor periphery to the avascular core. 
Interstitial hypertension

The interstitium is a collagen rich matrix and serves as a biological scaffold for tissues. It occupies the space between cells and tissues and is bordered by cell membranes and blood vessel walls. Within this matrix is a hydrophilic gel composed of interstitial fluid and macromolecular constituents such as proteoglycans and hyaluronate. Tumor interstitium differs greatly from that of normal tissues. Important distinctions include an interstitial space three to five times larger than that of normal tissues, high interstitial hydraulic conductivity and diffusivity, comparatively larger amounts of mobile fluid and faster spread of hydrophilic agents resulting from significant extravascular convection [19].

Leaky tumor vasculature and dysfunctional lymphatics in tumor interstitium result in undesirable accumulation of vascular contents in the tumor leading to interstitial hypertension [20,21]. Physico-chemical properties of the interstitium influence the movement of molecules between blood vessels and the cells. The interstitial pressure in normal tissues is approximately $0 \mathrm{~mm} \mathrm{Hg}$ while normal capillary pressure is around $1-3$ $\mathrm{mm} \mathrm{Hg}$. This pressure gradient facilitates convective transport of macromolecules from the vascular compartment to the interstitium. To the contrary, tumors display pressure gradients opposite to that of normal tissues. The presence of osmotic forces drawing solutes into tissue combined with functionally deficient tumor blood vessels and lymphatics as well as the ability of tumor stroma to contract all contribute to the higher interstitial fluid pressure within the tumor compared to blood vessels [21, 22]. Several studies have shown IFP of up to $100 \mathrm{~mm} \mathrm{Hg}$ in the tumor core while the periphery exhibits atmospheric like or slightly negative pressures [23-26]. Consequently, interstitial hypertension results in reduced convection across the walls of tumor blood vessels. Additionally, higher IFP in tumors favor the movement of interstitial fluid into surrounding tissues, flushing out therapeutic agents from the tumor. The combination of these two factors potentially hampers the potency of systemic therapies to tumors, especially delivery of macromolecules, since they depend on convective transvascular transport to cross the endothelial barrier and migrate through the interstitium [27].

\section{Low extracellular $\mathrm{pH}$}

For several decades tumors were generally believed to be acidic since cells actively convert glucose to lactic acid [28-31]. Using techniques that specifically measure intra- or extracellular $\mathrm{pH}$ in malignant tumors, it has now been shown that intracellular $\mathrm{pH}$ in tumor cells is neutral to alkaline while the extracellular $\mathrm{pH}$ is acidic [32-34]. This resulting $\mathrm{pH}$ gradient established across tumor cell membranes is preserved by a number of ion pumps which export protons into the extracellular compartment [35]. Aside aerobic and anaerobic glycolysis, other mechanisms have also been implicated in the tumor acidosis. Some of these pathogenetic mechanisms include ATP hydrolysis, glutaminolysis, ketogenesis and $\mathrm{CO}_{2}$ / carbonic acid production [36-38]. The $\mathrm{pH}$ gradient peculiar to tumors facilitates the accumulation of weakly acidic drugs (e.g., mitomycin $\mathrm{C}$ ) within tumors and may be utilized as a strategy for treating cancer [39, 40]. 
One such approach involves polymeric micelle delivery systems designed to release drugs when they encounter the acidic environment in tumors. These systems are stable at neutral $\mathrm{pH}$ but destabilize under mild acidic conditions ( $\mathrm{pH}$ 4.5-6.0) and facilitate release of anticancer drugs. $\mathrm{pH}$ sensitive drug carriers are typically designed by introducing acid labile chemical bonds between drug and carriers (Figure 2-2) [41, 42]. For instance, Bae and coworkers have utilized $\mathrm{pH}$-sensitive polymers such as poly (1histidine) as components in amphiphilic copolymers for micellar delivery [43-45]. Poly(1histidine $)\left(\mathrm{pK}_{\mathrm{b}} \sim 7.0\right)$ has an imidazole ring with lone electron pairs associated with the unsaturated nitrogen that confer it with $\mathrm{pH}$-dependent amphoteric properties . In one study, Bae et al., developed a $\mathrm{pH}$-sensitive mixed micelles system using poly(l-histidine) (polyHis) (Mw 5000)-b-poly(ethylene glycol) (PEG) (Mw 2000) and poly(l-lactic acid) (PLLA) (Mw 3000)-b-PEG (Mw 2000)-folate (0-25 wt.\%). At pH lower than 7.0, the poly(l-histidine) block ionized leading to a gradual disintegration of the system . When doxorubicin was incorporated, their studies showed $32 \mathrm{wt} \%$ of doxorubicin (DOX) was released at $\mathrm{pH} 7.0,70$ wt. $\%$ of DOX at pH 6.8, and 82 wt.\% at pH 5.0 in 24 hours [43]. Furthermore, it has been demonstrated that $\mathrm{pH}$-triggered micelle dissociation may enhance extravasation of subsequent micelles by creating space for the newly arriving micelles [46].

\section{Hypoxia}

From a pathophysiological standpoint, hypoxia refers to the condition in which tissues are deprived of oxygen or the partial pressures of oxygen falls below critical levels leading to hindered clinical and biological functioning of cells or organs [47]. For normal tissues, consumption of $\mathrm{O}_{2}$ for metabolic requirements is equaled by supply. In contrast, $\mathrm{O}_{2}$ demand far exceeds supply in tumors as they grow. High cell density caused by excessive rates of cell proliferation in tumors places a huge demand on local supply of oxygen. Furthermore, abnormal tumor vasculature (characteristic of tumors) reduces blood flow and limits delivery of oxygen to throughout the tumor resulting in regions of hypoxia. A major consequence of tumor hypoxia is resistance to chemotherapy [48].

Several factors contribute to hypoxia in tumors (Figure 2-3) and the different types of hypoxia are characterized accordingly. These include: inadequate perfusion (ischemic or acute hypoxia), increased diffusion distances (chronic hypoxia), anemia (anemic hypoxia) and hypoxemia (hypoxemic hypoxia). Generally, 50-60\% of solid tumors display heterogeneous distribution of hypoxic regions within the tumor. However, despite the type of hypoxia, controlling oxygen consumption within tumor cells is equally important as reduced oxygen delivery in determining tumor hypoxia. Hypoxia inducible factor-1 (HIF-1) plays a key role in tumor progression under hypoxia. 


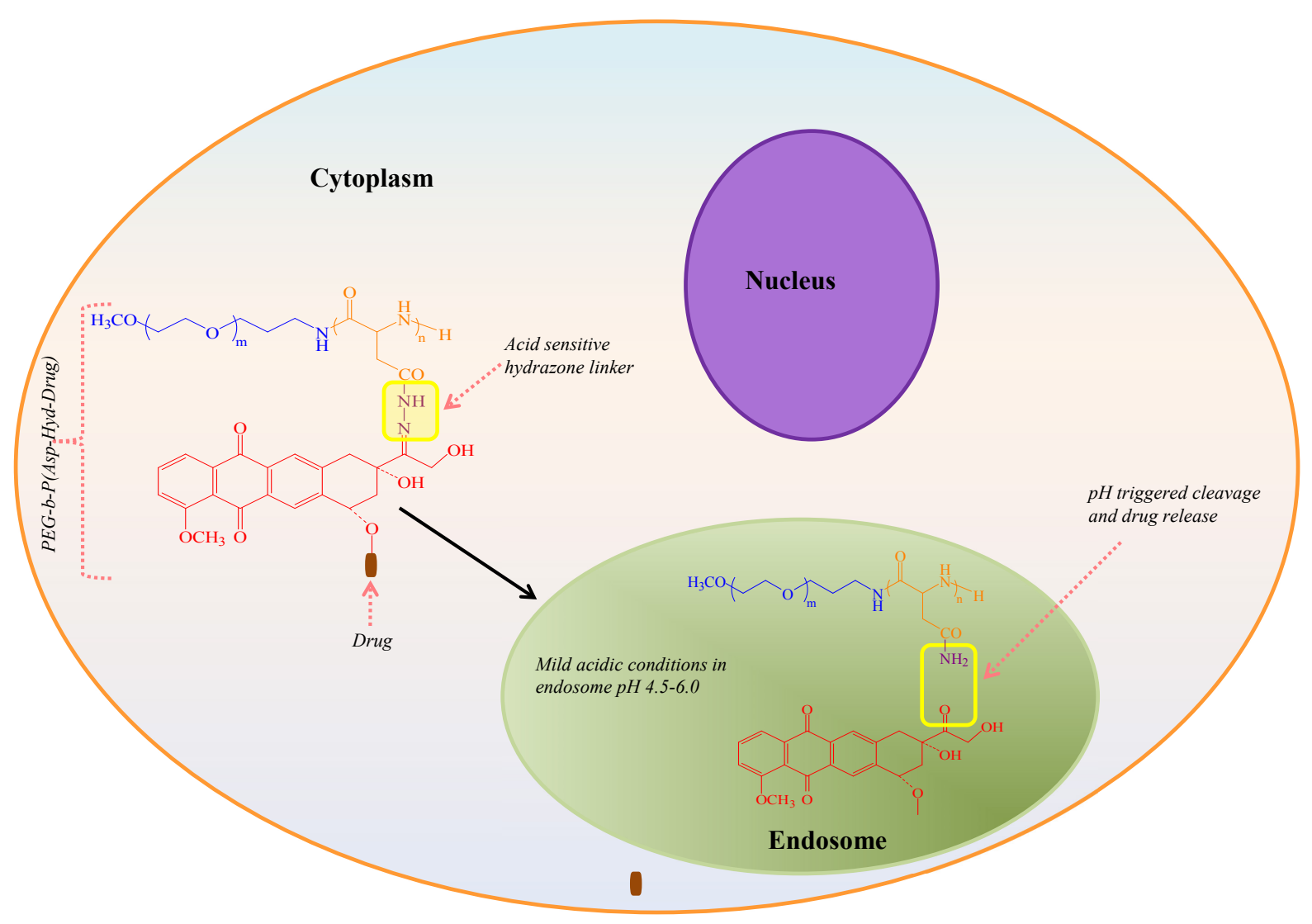

Figure 2-2. Schematic depicting pH triggered cleavage and drug release of poly (ethylene glycol)-poly(aspartate-hydrazonedrug) delivery system in acidic endosomal compartment.

Modified with permission from Bae Y, Jang WD, Nishiyama N, Fukushima S, Kataoka K. Multifunctional polymeric micelles with folate-mediated cancer cell targeting and $\mathrm{pH}$-triggered drug releasing properties for active intracellular drug delivery. Mol Biosyst. $2005 ; 1: 242-50$. 


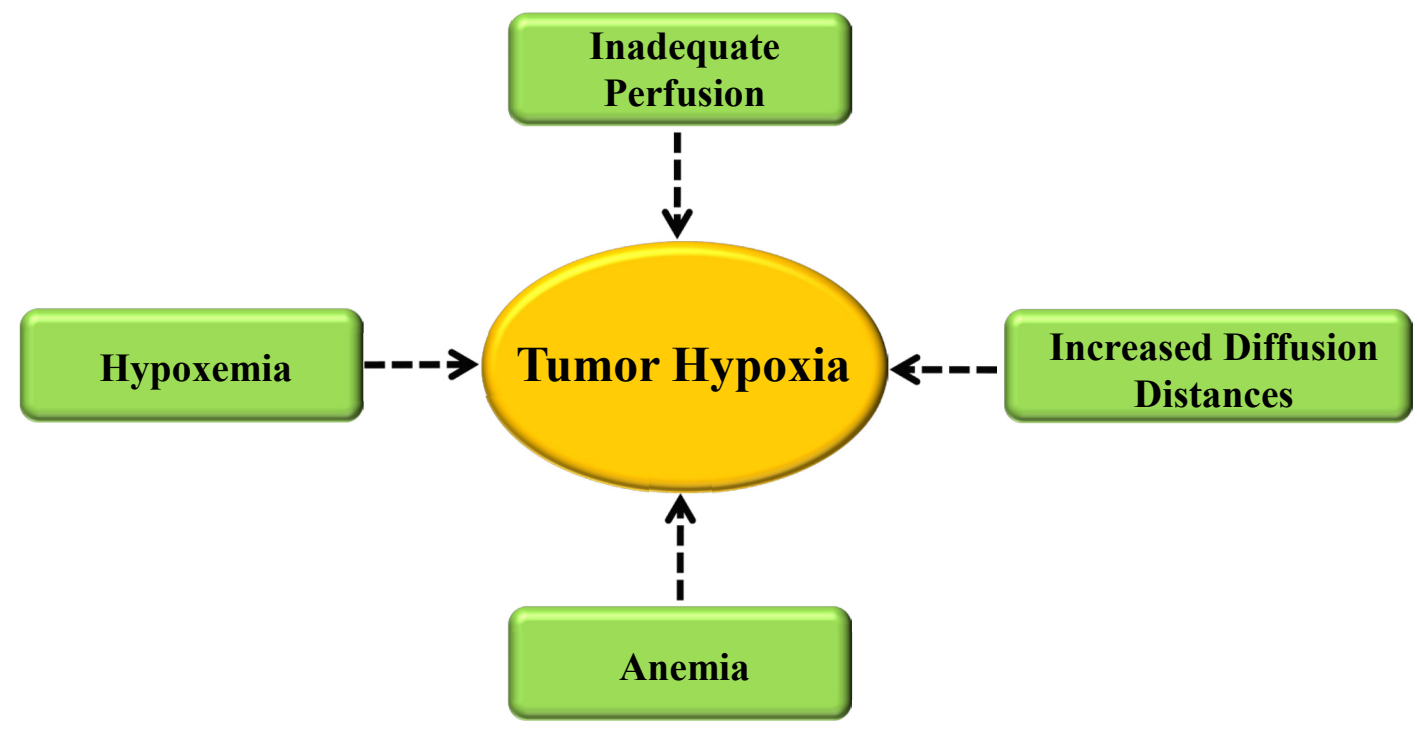

Figure 2-3. Crucial factors contributing to hypoxia in tumors. 
Angiogenesis

Tumor growth and metastasis requires the formation of new blood vessels from pre-existing vessels. This process is known as angiogenesis. Tumor microenvironment is replete with pro-angiogenic factors originating from neoplastic, stromal and infiltrating cells which affect angiogenesis. Tumor angiogenesis is characterized by an abundance of functionally defective and structurally abnormal blood vessels and occurs due to a disparity between the pro-angiogenic and anti-angiogenic factors. A consequence of these defective vessels is an inflamed tumor environment which encourages thrombosis and impaired blood supply resulting in hindered drug delivery to the tumor. Vascular endothelial growth factor (VEGF)/vascular permeability factor (VPF) is one of the most important angiogenic factors responsible for inducing proliferation and migration of endothelial cells and increasing vascular permeability [49]. Recently, VEGF was found to negatively regulate the maturation of blood vessel and the function of pericytes [50]. Other pro-angiogenic factors such as transforming growth factor- $\beta$ (TGF- $\beta$ ), interleukin (IL)-8 and basic fibroblast growth factor (bFGF) are produced as tumors begin to grow [51]. The key role blood vessels play in tumor growth and metastasis makes tumor angiogenesis a rational and well studied target for cancer therapy.

Tumor-stromal cell interaction

Tumor microenvironment consists of tumor cells embedded within the stroma. The tumor stroma is composed of a variety of normal cell types actively recruited by the tumor to provide the blood supply through the production and secretion of cytokines and stimulatory growth factors. A large number of T cells and macrophages are part of the tumor stroma. Tumor rejection partly depends on T cell activation. Tumor-infiltrating macrophages secrete angiogenic factors useful for tumor growth and inhibition of macrophage infiltration resulting in tumor regression. A thorough understanding of interactions between different stroma cell components that either support tumor growth or tumor rejection is important since this interaction is crucial to cancer development at the primary site and metastasis.

\section{Cancer stem cells}

Like normal adult tissues, solid tumors contain a population of cells that selfrenew and resupply the tumor with the various cell lineages of which it is composed of. These cells known as cancer stem cells (CSCs) are associated with tumor stromal components such as fibroblasts and blood vessels. They compose the cancer stem cell niche from which factors that support CSC renewal are released. CSCs form approximately $1 \%$ of the tumor. However, since they are the only cells that can maintain tumor growth indefinitely due to their self-renewal properties, they are the actual driving force behind tumor growth. The rest of the cells in the tumor microenvironment vigorously proliferate, differentiate and eventually die. 
It is known that CSCs exhibit similar properties to normal stem cells. Among these, CSCs are hypothesized to be relatively quiescent and have protracted life span, are refractory to apoptosis and resistant to toxins and drugs since they express at least one ATP-binding cassette (ABC) efflux transporter [52-55]. Since these drug efflux pumps occur naturally in CSCs, this hypothesis offers an additional mechanism by which tumors acquire multidrug resistance. A number of studies confirm this hypothesis. Recently, $\mathrm{Hu}$ et al. have shown a side population of ovarian cancer cells to possess ABC transporters and be drug resistant [56]. These cancer stem-like cell side populations were found to be responsible for tumorigenesis and grew more rapidly in xenogeneic transplant mice compared to ovarian cancer cells without these side population cells. Furthermore, drug efflux transporters have also been shown to be present in leukemic stem cells making them resistant to chemotherapeutic agents such as daunorubicin $[57,58]$. The purported role of CSCs in tumorigenesis and metastasis suggests targeting CSCs to be a potential therapeutic strategy for chemoprevention and cancer therapy.

\section{Abnormal lymphatics}

The lymphatic network transports interstitial fluid and immune cells out of normal tissue and is essential for immune function and maintenance of fluid balance in tissue interstitium. In contrast to normal tissues, the lymph vessels in tumors are compressed by solid stresses induced by tumor cells within the microenvironment [59]. Therefore, the functional performance of lymphatic vessels within the tumor microenvironment depends on their location. Lymphatic vessels situated at the periphery of the tumor or the stromatumor interface possess functionality while those within the tumor are functionally defective $[60,61]$. Lymphangiogenesis is facilitated by similar factors responsible for angiogenesis such as VEGF, angiopoietins and platelet-derived growth factor (PDGF). For instance, lymphangiogenesis and angiogenesis can be triggered by VEGF-C and -D and have been implicated in increased lymphatic metastases in numerous tumors [62, 63]. Abnormal lymphatics encourage retrograde flow in lymphatic vessels, allow tumor cells to invade the periphery of lymphatic vessels and promote metastases within the lymphatic system $[64,65]$.

\section{Anatomical Pathways of Macromolecular Extravasation}

The efficacy of blood borne therapeutic agents in cancer therapy depends on their extravasation across tumor blood vessels. Significant differences exist between the mechanism of extravasation of small molecules and macromolecules. However, the extent of extravasation in both cases is influenced by the macroscopic arrangement and wall structure of tumor microvessels. Also, the endothelial cell lining of microvessels function as a rate-limiting barrier for extravasation of plasma components. In normal tissues, transvascular transport of plasma water and small solutes (less than $2 \mathrm{~nm}$ ) occurs through gaps between adjacent cells [66]. However, since macromolecules have comparatively larger sizes they are unable to pass through these gaps. Although microvessels are more permeable than normal vessels, it has been shown that additional 
anatomical pathways are responsible for movement of macromolecules across blood vessels in normal tissues and tumors [67]. Presently, the dominant channels involved in macromolecule extravasation include inter- and trans-endothelial openings (open gaps), fenestrations and vesiculovacuolar organelle mediated extravasation [68-70]. Therefore, understanding the macroscopic and microscopic arrangement of tumor vasculature and the pathways responsible for transvacular transport of macromolecules is crucial to how they can be exploited to maximize extravasation of polymeric nanomedicines and enhance drug delivery.

Macroscopic arrangement

For a tumor to grow beyond a few millimeters, new blood vessels are required to increase blood supply to meet metabolic demands of malignant and non-malignant cells. Tumor angiogenesis leads to a variety of peculiar inter- and intra- tumor vascular morphologies partly driven by growth patterns of cancer cells. Two ideal classifications of vascular macroscopic arrangement are peripheral and central vascularization [71].

In peripheral vascularized tumors, blood vessels are confined to the periphery which is well-vascularized compared to the avascular core. The absence of vascular volume in the core implies systemic agents extravasate poorly in the center of the tumor. Contrarily, for central vascularization, blood vessels are localized and form dendrimer like patterns in the tumor core. Typically, macroscopic arrangement of tumor vasculature represents various degrees of these categories. Regardless of the organization of microvessels in tumors, it is clear that heterogeneity in vascular distribution and low vascular volume pose serious barriers to drug delivery. Hence, approaches that facilitate vascularization of tumors may improve polymeric nanomedicine extravasation and cancer therapy.

Tumor blood vessel wall structure

Tumor blood vessels can be classified into nine categories as described by Warren [72]. These include: arteries and arterioles, non-fenestrated capillaries, fenestrated capillaries, discontinuous capillaries, blood channels without endothelial lining, capillary sprouts, post-capillary sprouts, venules and veins and arterio-venous anastomoses. Among the blood vessels, extravasation occurs primarily at the capillaries and postcapillary venules due to their thin walls and large surface area [73]. The capillary wall structure is composed of a single layer of endothelial cells surrounded by a basement membrane. Capillary walls are leaky and have a distribution of pores with diameters less than $100 \mathrm{~nm}$. Depending on the frequency of pores in the endothelial layer, capillaries can be classified as non-fenestrated, fenestrated and sinusoidal [73, 74]. 


\section{Continuous capillaries}

Non-fenestrated capillaries, also known as continuous capillaries, are commonly found in cardiac, skeletal and smooth muscles as well as the skin and brain. Due to the tight junctions and uninterrupted subendothelial basement membranes, transport of molecules across the continuous capillaries is negligible. Since these vessels have an intact endothelial basement membrane with pore size less than $2 \mathrm{~nm}$, small molecules, however, can extravasate. An important difference between normal and tumor vessels is that tumor vessels are dilated and do not contain basement membrane leading to seepage of plasma oncotic contents into the interstitium. Unlike peripheral endothelium, the endothelial layer of the brain microvasculature is the tightest endothelium and has virtually no fenestrations. Therefore, extravasation of macromolecules across the brain is particularly difficult due to the presence of continuous capillaries between the blood and brain interstitium. However, certain regions of the brain, such as choroids plexus and medion eminence are not part of the blood-brain barrier and are more permeable.

\section{Fenestrated capillaries}

Fenestrated capillaries possess a continuous basement membrane but have fenestrations of 40-60 $\mathrm{nm}$ in diameter in the endothelium [74]. Fenestrated capillaries are found in the kidney, small intestine and salivary glands. The basement membrane surrounding fenestrated capillaries reduces the extravasation of nanomedicines, whereas macromolecules or drug-polymer conjugates of less than $11 \mathrm{~nm}$ in diameter extravasate freely. In contrast, glomerular capillaries have fenestrae of 60-80 nm, which allow effective permeation of macromolecules or nanomedicines of less than $30 \mathrm{~nm}$ in diameter. Fenestrated capillaries abound in various tumors, are partly accountable of tumor vessel permeability and permit extravasation of macromolecules [75-77].

\section{Discontinuous capillaries}

Discontinuous or sinusoidal capillaries have fenestrations of approximately 150 $\mathrm{nm}$ and are common in the liver, spleen, bone marrow, lymph nodes and adrenal cortex. The basal membrane in sinusoidal capillaries is absent in the liver, but present in spleen and bone marrow. While liver sinusoids contain phagocytic kupffer cells, those of bone marrow contain phagocytic reticuloendothelial cells and the spleen contains a large number of pinocytic vesicles.

\section{Endothelial openings}

It is widely believed that the increased permeability of tumor vessels is due to the presence of endothelial openings. Evidence provided by Majno and coworkers suggested these endothelial openings to be intercellular in nature [78]. These openings occur when neighboring endothelial cells sufficiently contract to overcome cell-cell and cell-matrix 
adhesion forces and pull away from each other. Using scanning electron microscopy (SEM), McDonald et. al. have shown that inter-endothelial openings are not simply holes in tumor vasculature. From their studies, they demonstrated inter-endothelial openings to be of two types: vertical gaps and oblique slits [79]. These pores range from 0.1 to $3 \mu \mathrm{m}$ in diameter and have been shown to occur 100 times more frequently than transendothelial openings [70, 79]. Hence, this has led to the long-standing assumption that inter-endothelial openings may account for abnormal leakiness of tumor vessels. Although the mechanism for pore formation and closure is still not well understood, vascular endothelial growth factor (VEGF)/ vascular permeability factor (VPF) and other cytokines have been shown to induce pores in endothelium that otherwise possessed no gaps $[68,80]$.

Vesiculo-vacuolar organelle mediated extravasation

The other competing hypothesis proposes extravasation via interconnected vesiculovacuolar organelles (VVOs). Dvorak and coworkers have demonstrated that VVOs are a major route of macromolecule extravasation at VEGF/VPF and tumorderived cytokine augmented hyperpermeable sites [81]. VVOs are morphologically similar to bunches of grape-like clusters approximately 50 to $415 \mathrm{~nm}$ in diameter and are frequently deployed at intervals in the cytoplasm of endothelial cells [82-84]. In one study where macromolecular tracers (e.g., anionic ferritn) were injected intravenously in TA3/St mammary carcinoma bearing mice, these tracers were found in VVOs within seconds and the VVOs provided a transcytotic pathway for the macromolecules to extravasate from tumor blood vessels [82]. Regardless of the controversy surrounding the dominant pathway for macromolecule extravasation, the consensus is that extravasation pathways for macromolecules and nanoparticles are channel-like structures [85].

\section{Extravasation across Microvascular Wall}

Solute transport across vessel walls is either diffusion or convection controlled (Figure 2-4). Diffusion is a function of vessel surface area and the driving force for this mode of transport is the concentration gradient established by plasma and interstitial concentration differences. Convection on the other hand is governed by the rate of fluid flow which in turn is determined by a pressure gradient. This pressure gradient is the difference between the resultant hydrostatic pressure (i.e., vascular minus interstitial) and the resultant osmotic pressure. The vascular permeability $(\mathrm{P})$ constant relates the diffusive mass flux to the concentration gradient while the hydraulic conductivity $\left(\mathrm{L}_{\mathrm{p}}\right)$ relates fluid flux to pressure gradients. For transport of macromolecules in normal tissue the primary mode of transport is convection, whilst that for small molecules is diffusion [86]. However, the mechanism of macromolecule extravasation in tumors is different due to high interstitial fluid pressure and leaky vasculature. Paradoxically, macromolecules generally exhibit poor extravasation in tumors although their blood vessels are leakier compared to normal tissue. This is due to the dominant effect of interstitial hypertension reducing fluid movement and consequently convective transport, thus overcompensating 


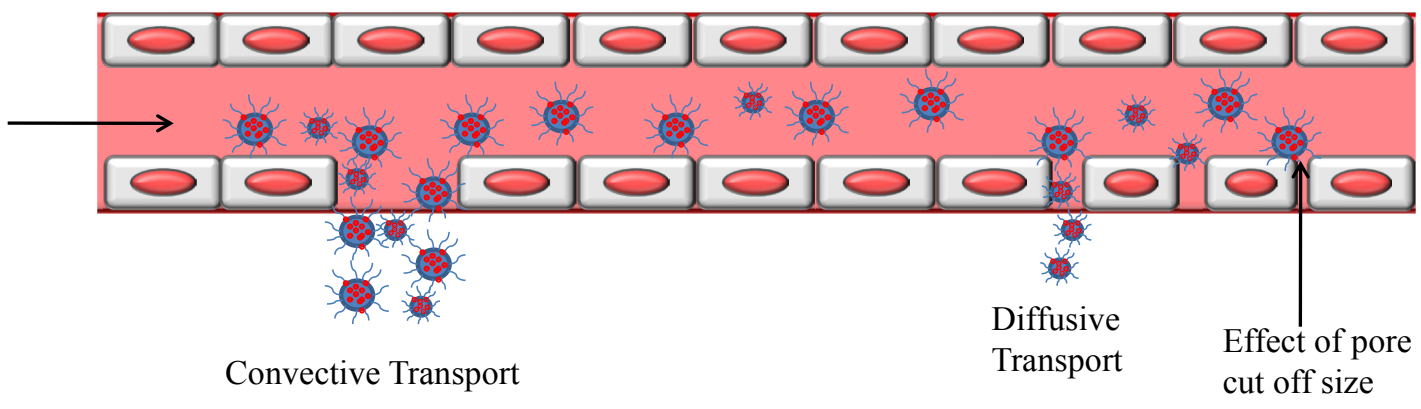

Figure 2-4. Schematic representation of convective and diffusive transport of polymeric nanomedicines across microvascular wall and the effect of pore cut off size. 
for the potential enhanced extravasation which may result from the leaky vasculature. Since the pressure gradient progressively gets steeper from tumor periphery to the core, extravasation of macromolecules is superior in the tumor periphery as against the tumor core [87]. For instance, Jain and colleagues showed higher accumulation of monoclonal antibodies at the tumor normal tissue interface [18].

\section{Barriers to Drug Delivery across Tumor Capillaries}

As alluded to above, a number of important factors related to the pathophysiology of tumors limit optimal drug delivery. These barriers vary depending on tumor type and microenvironment. The key then in successfully utilizing polymeric nanomedicines for treating solid tumors is to overcome or exploit these obstacles. Below we highlight some important barriers which hinder drug delivery and extravasation.

\section{Chaotic blood supply}

The first barrier is the chaotic blood supply to tumors. Significant structural and functional differences exist between tumor and normal vessels. In contrast to well organized microvessels in normal tissue, tumor vessels are frequently saccular, dilated and tortuous [73, 88, 89]. Their distribution, density, diameter as well as blood flow rate also differ throughout tumor. Presently, it is still not clear the relationship between the number of blood vessels in the tumor and its volume. For example, Jain and collaborators report a decrease in vascular volume while others report a constant vascular fraction over the tumor life span. Typically, tumors have a well-vascularized periphery, a seminecrotic core and an avascular necrotic central region. Hence, the extravasation of polymer therapeutics is often restricted to the tumor periphery. Polymeric nanomedicines however need to be sufficiently perfused to optimally deliver drugs to tumors. As such attention has been given to normalizing the tumor vasculature to enhance perfusion and the consequent extravasation of polymeric nanomedicines into tumors [90, 91].

\section{Poor permeability of tumor vasculature}

The next barrier is the poor permeability of tumor vasculature. Permeability of tumor blood vessels vary based on the type and location of tumors, leading to heterogeneous distribution of blood-borne therapies $[68,92,93]$. Hence, it is possible that tumor vessels in different locations (or in a similar microenvironment within the same tumor) display contrasting degrees of vascular leakiness. This structural feature often results in erratic interstitial drug delivery.

Blood brain barrier (BBB) consists of endothelial cells, pericytes, astrogline and macrophages and serves as a physical barrier to drug permeability to the brain. Drug permeability across BBB greatly depends on its octanol/water partition coefficient. Drugs with high octanol/water partition coefficient easily extravasate the BBB. However, some 
drugs with low octanol/water partition coefficient can also permeate BBB due to active or facilitated transport, which some substances with high octanol/water permeability poorly penetrate the BBB due to active transport back to the blood [94].

Limited transport across the interstitium

The third barrier relates to the ability of drug delivery systems to traverse the interstitium and selectively target and eliminate cancerous cells. Two key issues here are interstitial transport and interstitial hypertension. Size, charge, shape and physicochemical properties of the interstitium are important parameters governing interstitial transport. For example, large size hinders tissue penetration of macromolecules, while cationic liposomes have been shown to target the intravascular tumor compartment better than anionic and neutral liposomes of similar size $[95,96]$. Hence, studies elucidating the effects of size, charge and shape of macromolecules on their transport in a variety of tumor types and locations are important in overcoming this obstacle. Interstitial hypertension creates a pressure gradient favoring the movement of drug molecules out of the tumor into the vascular compartment [97]. This results in reduced macromolecular delivery and transport since convection is its major mode of transport [22].

\section{Multidrug resistance}

Multidrug resistance (MDR) is another important barrier limiting the success of chemotherapy and resulting in observed clinical drug resistance. MDR arises when cancer cells become insensitive and cross-resistant to cytotoxic effects of structural and functionally diverse anticancer agents targeting a range of distinct molecular targets [98]. Since MDR implies cancer cells are irresponsive to normal dosing regimen of chemotherapeutic drugs, higher doses and frequency are often required in the clinic. A major consequence of this is the detrimental side effects on otherwise healthy organs and tissues due to high dose intake of anticancer agents to obtain desired therapeutic effect. The mechanisms responsible for MDR and approaches for reversing MDR using polymeric nanomedicines are discussed below.

\section{Mechanism responsible for multidrug resistance}

The mechanisms underlying MDR may broadly be classified as pump or nonpump mediated [99]. Table 2-1 summarizes some of these mechanisms and therapeutic strategies for overcoming them. For pump mediated MDR, it has been shown that overexpression of members of the ABC transporter super-family such as p-glycoprotein (P-gp), multidrug resistance-associated proteins (MRP) and breast cancer resistance protein (BCRP) are responsible for increased cellular drug efflux [100-102]. These transporters compromise potency of chemotherapy when they reduce drug concentration within the cell after pumping penetrated anticancer drugs out of the cells cytoplasm. To 
Table 2-1. Multidrug resistance mechanisms.

\begin{tabular}{lll}
\hline Type of multidrug resistance & Mechanism & $\begin{array}{l}\text { Therapeutic agents and strategies } \\
\text { to overcome drug resistance }\end{array}$ \\
\hline
\end{tabular}

\section{Pump Mediated Mechanism}

Enhanced cellular drug efflux

Overexpression of ATP-binding cassette transporters (e.g., P-gp, MRP,BCRP)

\section{Non-Pump Mediated Mechanism}

Dysfunctional apoptotic pathway

Drug detoxification
Overexpression of antiapoptotic proteins (XIAP and Bcl-2)

Reduced sphingolipid ceramide generation or enhanced ceramide metabolism

Overexpressed glutathione Stransferase
Polymeric nanomedicines (e.g., Pluronics); MDR reversal agents (e.g., PSC833, XR9576, Ixabepilone, SMART compounds); Combination therapy (e.g., bicalumatide and embelin)

XIAP inhibitor (e.g., embelin), Bcl-2 inhibitor (e.g., ABT-737), antisense therapy (e.g., Genasense, G3139)

Ceremide generation activator (e.g., gefitinib); ceramide metabolism inhibitors (e.g., LCL204, $\mathrm{N}$-oleoylethanolamine)

$\gamma$ - Glutamylcysteine synthestase

Part of this table adapted with permission from Wong et al., Multidrug Resistance in Solid Tumor and Its Reversal, in R.I. Mahato, Y. Lu (Eds.), Pharmaceutical Perspectives of Cancer Therapeutics, Springer, New York, 2009, pp. 121-148. 
date, $\mathrm{P}-\mathrm{gp}$ is the most well studied and characterized ABC transporter. It is a $170-\mathrm{kDa}$ ATP-dependent glycoprotein capable of transporting a wide range of structurally and functionally different therapeutic moieties and hence causes cross-resistance to a host of drugs. Some P-gp substrates include taxanes (docetaxel, paclitaxel) and anthracyclines (doxorubicin, epirubicin) [103]. In contrast to P-gp, multiple MRP (MRP-1 to MRP-8) members are responsible for drug resistance in cancer with each member being highly specific for a particular substrate. Although BCRP was originally identified in breast cancer, it has been shown to be present in other normal tissues such as the colon [104]. However, BCRP is overexpressed in tumors and is highly resistant to mitoxantrone[105]. A key question is how $\mathrm{ABC}$ transporter MDR phenotype is conferred in solid tumors. In the case of P-gp, MDR phenotype has been shown to be native or acquired. For example, it is intrinsically expressed in epithelial cells of kidney, liver and pancreas and higherbasal expression is observed in cancers related to these organs [103]. P-gp MDR phenotype may also be acquired after short exposure to high doses or prolonged exposure to sub-therapeutic levels of anticancer agents.

Although overexpression of ABC transporters may be considered the primary cause of MDR, other non-pump mediated mechanisms prevent cancer cell death and lead to drug resistance. Most of these mechanisms result in reduced drug activity due to dysfunctional molecular pathways. For example, a defect in the apoptotic machinery leading to overexpression of antiapoptotic proteins (e.g., BCl-2, XIAP) does not prevent intracellular drug accumulation but rather simply prevents cells from undergoing programmed cell death. Also, Minko and coworkers have shown that doxorubicin treated cancer cells resulted in overexpression of glutathione-S and UDP transferases [106]. These two proteins detoxify drugs and reduce drug efficacy in tumors. Additionally, the structural complexity of solid tumors poses multiple pharmacokinetic and pharmacodynamic barriers which lead to drug resistance. Some examples include loss of drug activity in the acidic tumor microenvironment due to deactivation and acquisition of drug resistance mechanisms in cancer cells located in hypoxic regions of tumors.

\section{Reversing multidrug resistance using polymeric nanomedicines}

Polymeric nanomedicines are well suited for overcoming MDR in a number of ways. First, the delivery vehicles themselves can hinder the proper functioning of transporters [107]. Recently, it has been hypothesized that block-copolymers such as pluronics may disturb the configuration of the lipid bilayer anchoring $\mathrm{ABC}$ transporters and consequently hinders their ability to pump drugs out of the cell [108, 109]. In addition, it has been suggested that pluronics decrease enzymatic activity of glutathione/glutathione $S$-transferase (GSH/GST) and hence decrease drug detoxification by these enzymes [110]. Furthermore, pluronics tilt the balance of the apoptotic machinery in favor of pro-apoptotic signaling by reducing mitochondrial membrane potential of MDR cells and facilitating cytochrome $C$ release [111]. Experimental evidence demonstrates the validity of these hypotheses. Recently, Batrakova et al., have shown that pluronics P85 can enhance accumulation of P-gp substrates in tumors and promote apoptosis in preclinical mice models bearing leukemia and Lewis lung MDR 
tumors [112]. In another study, $\varepsilon$-caprolactone modified poloxomer 188 nanoparticles containing paclitaxel increased uptake in $\mathrm{MCF}-7$ paclitaxel resistant human breast cancer cell lines [113].

Second, MDR reversal agents may be encapsulated in polymeric nanomedicines to facilitate drug delivery and overcome MDR. Since most MDR reversal agents are lipophilic and have other unfavorable physicochemical properties, they can be more selectively and efficiently administered to target sites using lipophilic-based polymeric vehicles thereby improving efficacy. In a recent study, D- $\alpha$-tocopheryl polyethylene glycol 1000 succinate (TPGS, a known P-gp modulator)-poly lactide (PLA) nanoparticles was used to efficiently encapsulate and deliver doxorubicin to drug resistant breast cancer cells (MCF-7/ADR). This system resulted in increased inhibition of P-gp activity, cell proliferation and enhanced intracellular drug accumulation and nuclear localization [114]. We have also used PEG-PLA copolymer to significantly increase the solubility of a novel microtubule destabilizer methoxybenzoyl-ary-thiazole (SMART-100) (Figure 2-5A) highly potent in a broad-spectrum of cancer cell types by up to 100,000 fold $[115,116]$. In addition, our studies revealed SMART-100 to be more potent in drug resistant prostate cancer cells (DU-145-TXR) compared to paclitaxel (Figure 2-5B and C).

Finally, combination therapy using multiple anticancer drugs is a promising approach to overcome drug resistance. This strategy appears to outsmart cancer cells since it becomes difficult for them to simultaneously develop multiple resistance mechanisms to counteract treatment. We have shown that combining bicalutamide (nonsteroidal antiandrogen) and embelin (XIAP inhibitor) (Figure 2-6A) leads to synergistic anticancer effects in hormone refractory human prostate cancer cells (C4-2) [11]. Our strategy involved concurrently targeting the androgen receptor and XIAP signaling pathways (Figure 2-6B) both of which are pivotal to progression and metastasis of prostate cancer. We found anticancer effect to be schedule dependent with simultaneous treatment being more efficacious than sequential administration (Figure 2-6C). Polymeric nanomedicines can be used to enhance efficacy of combination therapy in vivo as we demonstrated using PEG-PLA micelles. It has also been shown that polymer-lipid hybrid nanoparticles (PLN) used to administer doxorubicin and mitomycin-C inhibited colony-forming potential in drug resistant breast cancer cells [117].

Inappropriate animal models

While several animal models are presently used in studying drug delivery systems in cancer, these models are typically xenografts generated by injecting a variety of cell lines subcutaneously. Although adequate for proof of concept studies, the tumor microenvironment in xenografts is distinctly different from that in human tumors and does not truly reflect what pertains in humans. Orthotopic models are more appropriate since tumors are established in the organ of interest and can result in metastatic disease. However, these models often require invasive procedures and are difficult to establish (e.g., injecting tumor cells into the prostate of mouse models). Additionally, tumor growth and metastasis in orthotopic models cannot typically be visualized with the naked 


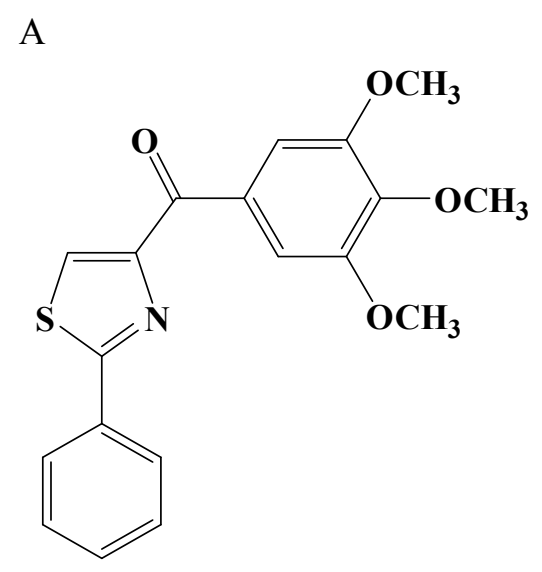

B
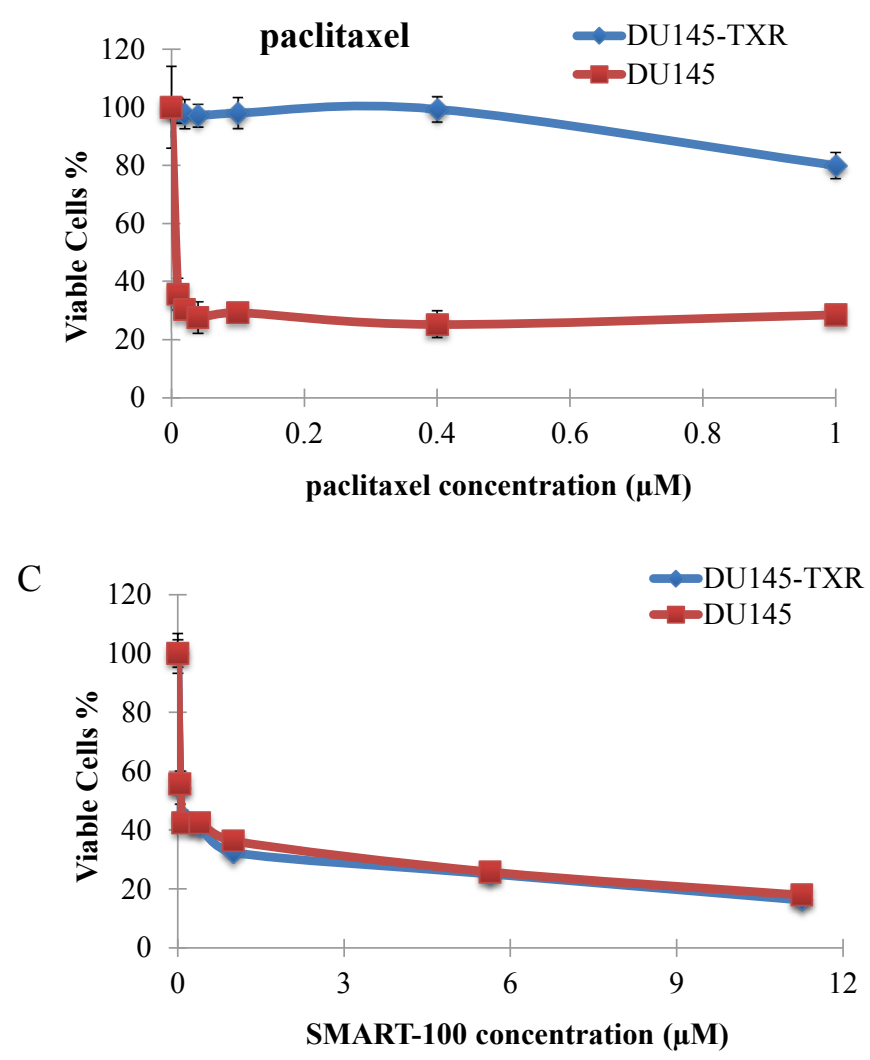

Figure 2-5. Chemical structure and anticancer effect of SMART-100.

(A) Chemical structure of SMART-100. (B) Anticancer effect of paclitaxel on DU-145 and DU145-TXR cell growth and (C) Anticancer effect of SMART-100 on DU-145 and DU145-TXR cell growth. SMART-100 is highly potent regardless of MDR status.

Reproduced with permission from Li F, Lu Y, Li W, Miller DD, Mahato RI. Synthesis, formulation and in vitro evaluation of a novel microtubule destabilizer, SMART-100. J Control Release. 2010;143:151-8. 

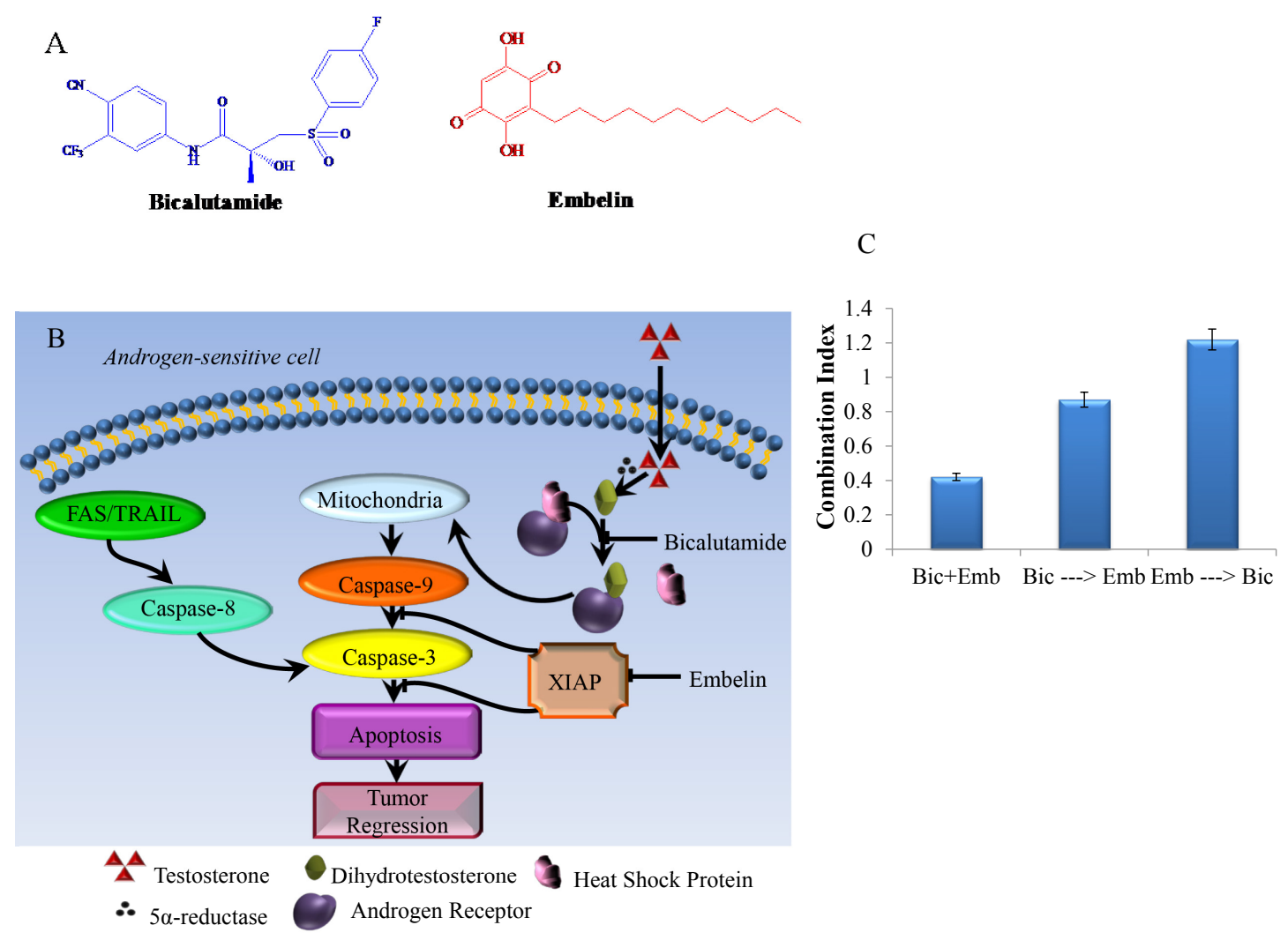

\section{Figure 2-6. Anticancer effect of antiandrogen and XIAP inhibitor based combination therapy.}

(A) Chemical structures of bicalutamide (Bic) and embelin (Emb). (B) Schematic diagram showing the combined effect of bicalutamide and embelin on apoptosis in androgen-sensitive cells and on tumor regression and (C) Combination Index (CI) of simultaneous and sequential treatment of bicalutamide and embelin in C4-2 cells. $5 \times 10^{3}$ cells were simultaneously treated with a combination of bicalutamide and embelin or sequentially [(bicalutamide followed by embelin) or (embelin followed by bicalutamide)]. Combination index (CI) was calculated by the formula: $\mathrm{CI}=$ $\left(\mathrm{d} 1 / \mathrm{D}_{50} 1\right)+\left(\mathrm{d} 2 / \mathrm{D}_{50} 2\right)$, where $\mathrm{D}_{50} 1$ is the dose of bicalutamide required to produce $50 \%$ effect alone, and $\mathrm{d} 1$ is the dose of bicalutamide required to produce the same $50 \%$ effect in combinationwith $\mathrm{d} 2 . \mathrm{D}_{50} 2$ is similarly the dose of embelin required to produce $50 \%$ effect alone, and $\mathrm{d} 2$ is the dose ofembelin required to produce the same $50 \%$ effect in combination with d1. $10 \mu \mathrm{M}$ bicalutamide was combined with $0,5,10,25$ and $50 \mu \mathrm{M}$ embelin. The CI values are interpreted as follows: $>1.0$, synergism; 1.0, additive; and $<$ 1.0, antagonism. Reproduced with permission from Danquah M, Li F, Duke CB, 3rd, Miller DD, Mahato RI. Micellar delivery of bicalutamide and embelin for treating prostate cancer. Pharm Res. 2009;26:2081-92. 
eye. However, orthotopic tumor growth can now be monitored using current technologies such as bioluminescence and microultrasound.

\section{Strategies for Enhanced Extravasation}

To improve the therapeutic potential of polymeric nanomedicines, they should be designed to bypass at least one of the physiological barriers described above. To date, approaches taken to overcome these barriers have relied on either targeting the tumor vasculature or lowering interstitial hypertension. A few of these strategies are highlighted below.

Targeting the tumor vasculature

From a therapeutic standpoint, tumor blood vessels are excellent targets due to their peculiar features which distinguish them from normal blood vessels. Furthermore, they lack the genetic stability which facilitates the development of drug resistance in cancer cells. Extravasation of polymeric nanomedicines can be improved by using radiation to further increase the permeability of the tumor vessels. This can be done using an external source or radioisotope targeted macromolecules. To date, tremendous effort has been channeled using this approach to investigate the effects of radiation tumor blood flow and vascular volume. Typically, scientists have reported on the extent of macromolecule extravasation in one hour or the effects of radiation on the time course of tissue uptake. Potchen and coworkers [118] determined tissue pharmacokinetics of albumin in Gardener lymphosarcoma tumors, skin, muscle and plasma in $\mathrm{C} 3 \mathrm{H}$ mice 48 hours after radiation ( $3000 \mathrm{R}$; single dose). The tissue to plasma ratio increased by $\sim 200 \%, 80 \%$ and $70 \%$ in tumor, muscle and skin, respectively by 48 hours after albumin injection. The authors also observed no significant increase in tumor vascular volume (using labeled RBCs). From these results, they surmised that radiation resulted in an increase in vascular permeability in tumors.

In another study, Song and coworkers [119-121] investigated the quantity of albumin extravasated in 1 hour, and vascular volume, measured using labeled RBCs in three tumors: Walker 256 carcinoma in Sprague-Dawley rats; a neuroblastoma in A/J mice; and the SCL carcinoma in A/J mice, and observed a tumor dependent response. They found that quantity of albumin extravasated in 1 hour increased in W256 2 days after $200 \mathrm{R}$ and 1 day after $3000 \mathrm{R}$ without an increase in vascular volume. However, both the quantity of albumin extravasated in 1 hour and vascular volume decreased for all doses thereafter. Similarly in the neuroblastoma, quantity of albumin extravasated in 1 hour increased immediately after radiation with doses of 250--20001R, and decreased after 1-2 days. Vascular volume did not change significantly in the beginning; however, it decreased from day 3 after 250--500 R and from day 1 after 1000-2000 R radiation. The response of SCL tumor was entirely different. This tumor showed a short term (1 day) increase followed by a slight decrease $(\sim 25 \%)$ in vascular volume 7 days after 1000 $2000 \mathrm{R}$ radiation. The tissue uptake, quantity of albumin extravasated in 1 hour, remained 
fairly constant and decreased by $<20 \%$ in 7 days after radiation. From these experiments, the authors' surmised that the decrease in quantity of albumin extravasated in 1 hour probably resulted from a decrease in vascular volume/surface area due to radiative reorganization and disturbance of the vascular network.

Hyperthermia can also boost tumor blood vessel permeability and lead to enhanced drug delivery which could possibly translate into better efficacy [122-125]. To date, elevated temperatures have been shown to increase the permeability of tumor vessels to ferretin [126], Evans blue dye [127], antibodies [128-130], and liposomes [122, 131]. Recently Kong et al. showed mild heating of tumors to confer the most benefit for nanoparticle based drug delivery [131]. Several scientists have tried to elucidate the mechanism by which hyperthermia affects vascular permeability. For example, Wilhelm and Mason [132] explained the observed thermal induced increase in vascular permeability to be due to the liberation of endogeneous vasoactive agents such as bradykinin, histamine, and serotonin.

Another approach to targeting tumor vasculature involves coupling chemotherapeutic drugs to ligands (antibodies, peptides and proteins) that bind specifically to markers suchs as aminopeptidase-N, $\alpha v \beta 3$ and $\alpha v \beta 5$ overexpressed on tumor vasculature [133]. For example, pro-apoptotic peptides and tumor necrosis factor- $\alpha$ coupled to peptides targeting the above-mentioned such markers include have been shown to reduce tumor growth in vivo [133-135].

Lowering tumor interstitial hypertension

Since interstitial hypertension correlates with inefficient extravasation of macromolecules it is an attractive target for improving transvascular transport. One way this may be achieved is by using drugs designed to increase systemic blood pressure or reduce contractility of extracellular matrix $[21,136]$. Currently, the use of VEGF inhibitors such as bevacizumab or anti-VEGF antibody is a popular approach that has proved potent in animals and patients [137]. VEGF is overexpressed in tumors and known to account for leakiness in tumor vasculature [138]. In one study, a monoclonal antibody against VEGF was used to treat mice bearing subcutaneous human glioblastoma tumors and it was found to reduce tumor IFP by more than 70\% [139]. Tong and coworkers recently showed decreased interstitial hypertension caused by targeting VEGF to result in pressure gradients favoring extravasation and hence improving drug penetration in tumors [90]. Similar results have been demonstrated in clinical studies where decreased IFP was observed in patients with rectal carcinomas upon treatment with bevacizumab and when combined with 5-fluorouracil and leucovorin significantly improved patient survival $[140,141]$. The enhanced therapeutic effect of VEGF inhibitors is considered to occur via normalization of tumor vessels resulting in reduced vascular permeability and consequently decreased IFP in tumors.

Tumor IFP can also be lowered using PDGF antagonists. Unlike VEGF inhibitors, PDGF antagonists target the interaction between stromal fibroblast and the extracellular 
matrix. Imatinib is a selective small-molecule PDGF receptor inhibitor which has been shown to lower IFP [142] and increase delivery and potency of taxol, 5-fluorouracil [143] and epthilone B [144] when used in combination. PDGF specific DNA aptamers have also been found to lower IFP in mice bearing KAT-4 thyroid carcinoma [143].

Transforming growth factor- $\beta$ (TGF- $\beta$ ) receptors antagonists have been found to lower interstitial hypertension by decreasing the amount of extracellular-matrix molecules [145] and also through normalization of tumor vessels [146]. Lammerts and coworkers observed that blockade of TGF- $\beta$ resulted in a dose-dependent decrease of IFP in mice bearing KAT-4 thyroid carcinoma. Interestingly, this effect was seen 5-10 days after administration of the TGF- $\beta$ inhibitor, suggesting that a reorganization of the tumor tissue was necessary to observe the effect. Furthermore, one study showed that the combination of a prostaglandin E1 and 5-fluorouracil resulted enhance therapeutic effect in rodent tumors by reducing IFP [147].

Finally, administration of anti-inflammatory and chemotherapy agents has been shown to lower IFP in tumors. For instance, dexamethasone was observed to lower IFP in xenograft human colon carcinoma LSI74T tumors. It was suggested that dexamethasone decreased the matrix molecule content and reduced the permeability of tumor blood vessels. Recently, paclitaxel was shown to reduce compression of tumor vessels leading to lower IFP in preclinical studies [148]. Taghian et. al. also showed in their experiments that breast cancer patients treated with paclitaxel displayed a lowering of interstitial hypertension. In contrast, treatment with doxorubicin did not reduce IFP [149]. Consequently, combination therapy utilizing paclitaxel in conjunction with other target specific chemotherapy drugs can improve therapeutic outcome in cancer patients.

Increasing aqueous solubility of potent anti-cancer drugs

As mentioned previously, low aqueous solubility of potent anticancer drugs is a major impediment to their delivery and efficacy. Polymeric nanomedicines are capable of increasing the aqueous solubility of lipophilic drugs and offer a number of important advantages over traditional solubilizing agents such as DMSO. First, they are less toxic than their traditional counterparts and consequently prevent adverse effects on healthy tissues. Adverse effects of anticancer drugs often result in termination of therapy or at best limit use to sub therapeutic doses. Second, polymeric nanomedicines can be actively targeted to make them site-specific. Targeting enhances drug uptake by cancerous cells, improves efficacy and further reduces adverse effects. Polymeric nanomedicines may broadly be classified as particulate carriers and bioconjugates. Below, we discuss polymeric micelles which are classic examples of particulate carriers and how they assist in enhancing drug solubility and extravasation. 


\section{Polymer-drug conjugates}

Polymer-drug conjugates are drug delivery systems composed of a drug chemically bound to a polymeric carrier by a biodegradable covalent bond. The attachment of drug to polymeric carrier can result in several important advantages compared to free drug which can potentially facilitate extravasation and cancer therapy. These include: (i) enhanced drug solubility, (ii) longer circulation times and consequent passive targeting [150], (iii) reduced toxicity [151], (iv) ability to bypass drug resistance [152] and (v) ability to be actively targeted [153]. However, to achieve such benefits, special attention should be given to a number of features required for effective design. For example, the polymer must be non-toxic and capable of decent drug loading to potency ratio. Also, the polymer-drug system should be stable whilst in transit to the tumor site but be able to release the drug in a desired manner once it reaches the tumor. To date several polymer-drug conjugates are in clinical trials [154, 155]. Most of these systems are designed for one drug moiety. However, polymer-drug conjugates for combination therapy are presently being actively designed to treat cancer [12].

\section{Polymeric micelles}

Polymeric micelles are nanosized self-assembled supramolecular core-shell spherical structures. The core is composed of the hydrophobic portion of the copolymer and is capable of solubilizing a considerable amount of highly water insoluble drugs. The hydrophilic component of the copolymer forms the shell (corona) which provides steric stabilization. They also have stealth properties which prevent recognition by the reticuloendothelial system (RES) and therefore minimize elimination of the micelles from the bloodstream [156]. Thus, these so called 'stealth' properties of the PEO shell result in increased blood circulation times and prevention of plasma protein binding [157]. In addition, the small size ensures preferential accumulation in tumor cells via the enhanced permeability and retention (EPR) effect $[150,158]$.

\section{Physics of drug encapsulation}

Polymeric micelles can enhance drug solubility by physical entrapment (encapsulation) or chemical conjugation. While chemical conjugation can result in high drug loadings, it is not suitable for all drugs since some therapeutic agents do not have functional groups necessary for conjugation and cannot be modified without altering pharmacologic effect. In contrast, physical entrapment is suitable for a wide range of drugs. However, the extent of drug encapsulation depends of compatibility between drug and core-forming block of micelle $[159,160]$ and core-forming block length $[161,162]$. The effect of these parameters is measured in terms of drug loading density (ratio of weight of encapsulated drug to weight of micelle) and drug loading efficiency (ratio of weight of encapsulated drug to weight of drug initially fed). High drug loading density and encapsulation efficiency are desirable to facilitate clinical translation of micelles since this translates into smaller amount of polymer and processing cost. 


\section{Compatibility between drug and core forming block}

Improvement in the extent of compatibility between a drug and the core forming block of the micelle may translate into superior encapsulation efficiency [163-166]. Polymer/drug compatibility may be characterized by Flory-Huggins interaction parameter $(\chi \mathrm{FH})$ which accounts for the forces of interaction between the polymer and the drug. The low $\chi \mathrm{FH}$ values suggest that the polymer is thermodynamically a good solvent for the drug. Flory-Huggins interaction parameter $(\chi \mathrm{FH})$ which characterizes polymer-drug compatibility is calculated using Equations 2-1 and 2-2:

$$
\begin{gathered}
\Delta=\left[\left(\delta_{d}-\delta_{p}\right)_{\text {polarity }}^{2}+\left(\delta_{d}-\delta_{p}\right)_{\text {dispersion }}^{2}+\left(\delta_{d}-\delta_{p}\right)_{\text {hydrogen }}^{2}\right]^{\frac{1}{2}} \\
\chi_{F H}=\frac{\Delta^{2} V_{d}}{R T}
\end{gathered}
$$

where $\Delta^{2}$ is the solubility difference between the drug (d) and the core of the polymeric micelle (p). $V_{d}$ is the molar volume of the drug, $T$ is the temperature in Kelvin and $R$ is the gas constant. $\left(\delta_{\mathrm{x}}\right)_{\mathrm{d}},\left(\delta_{\mathrm{x}}\right)_{\mathrm{p}}$ and $\left(\delta_{\mathrm{x}}\right)_{\mathrm{h}}$ are Hansen partial solubility parameters for the drug and the hydrophobic block of the block copolymer.

To design a micelle system with improved drug loading, it is prudent to first perform an in silico study to assess the Flory-Huggins interaction parameter $\left(\chi_{\mathrm{FH}}\right)$ between drug and the block copolymers. As $\chi_{\mathrm{FH}}$ approaches zero, compatibility between the polymer and the drug progressively increases since the polymer increasingly becomes a better thermodynamic solvent for the drug, resulting in improved drug solubilization.

Our group and others have explored the possibility of predicting drug solubilization in micelles based on thermodynamics and found our predictions to closely approximate experimental results [164, 167-171]. For example, we recently utilized this approach to match two anticancer drugs: bicalutamide and embelin with novel block copolymers. In one study, 5-methyl-5-benzyloxycarbonyl-1,3-dioxane-2-one (carbonate monomer) was introduced into the poly (ethylene glycol)-b-poly (L-Lactide) (PEG-bPLLA) copolymer backbone to form poly (ethylene glycol)-b-poly (carbonate-co-lactide) (PEG-b-(CB-co-LA)) copolymer. The chemical structure and molecular model of (PEGb-(CB-co-LA)) copolymer are shown in (Figure 2-7A and B). Our predictions based on $\chi_{\mathrm{FH}}$ values of PEG-b-(CB-co-LA) and PEG-b-PLLA copolymers indicated PEG-b-(CBco-LA) to be a better thermodynamic solvent for bicalutamide due to its lower $\chi_{\mathrm{FH}}$ value.

Experimental evidence confirmed this prediction since PEG-b-(CB-co-LA) increased drug loading of bicalutamide by up to four-fold (Figure 2-7C) and improved thermodynamic solubility by 10 fold (Figure 2-7D). It is noteworthy that both drug loading and thermodynamic stability were carbonate content dependent.

Using a dodecanol lipid grafted onto a poly (ethylene glycol)-b-poly carbonate (PEG-b-PBC) copolymer, we were able to increase loading efficiency of embelin from 

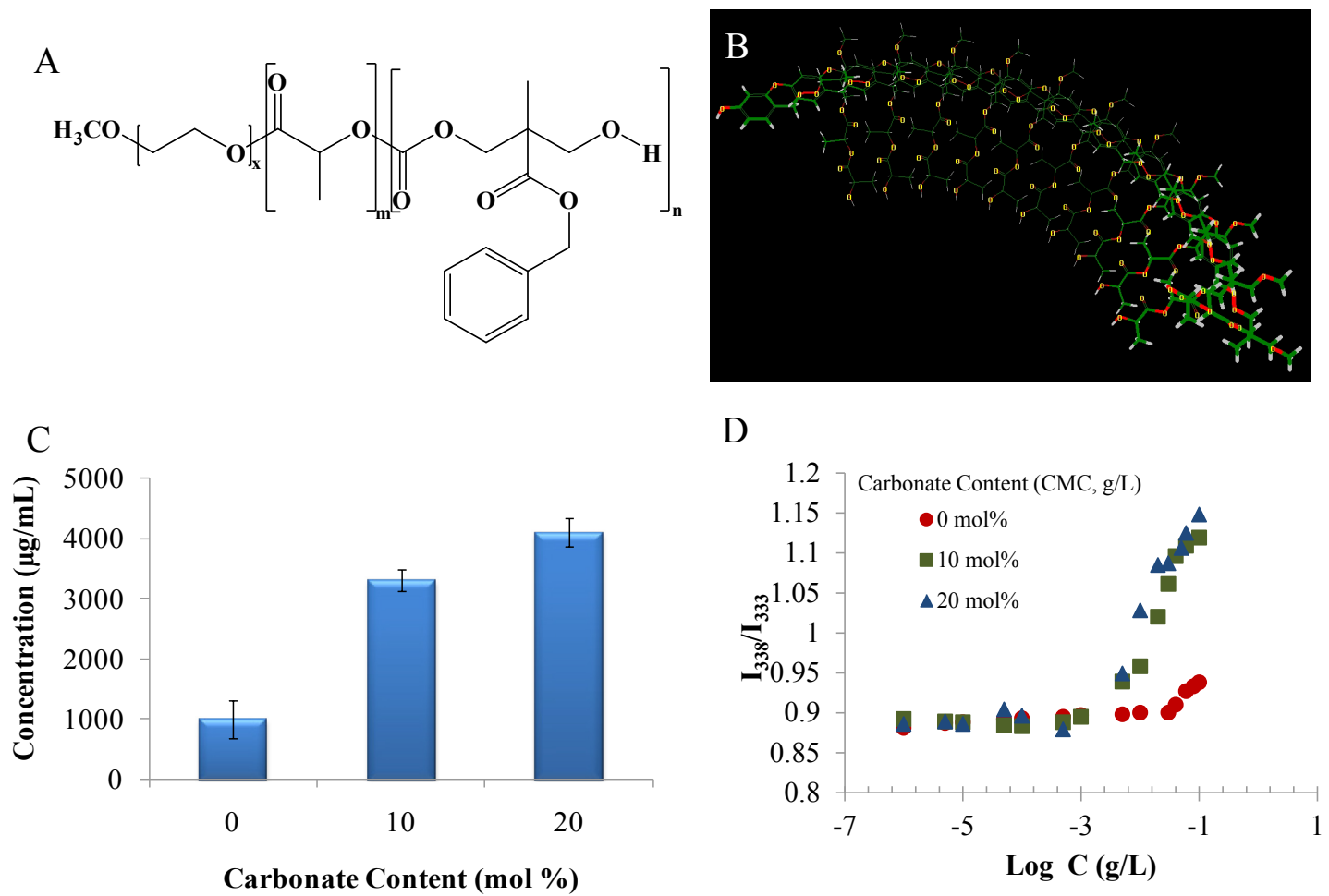

Figure 2-7. Effect of carbonate content on key poly (ethylene glycol)-b-poly (carbonate-co-lactide) micelle properties.

(A) Chemical structure of poly (ethylene glycol)-b-poly (carbonate-co-lactide).

(B) Molecular modeling of PEG-b-P(CB-co-LA) block copolymer; molecular modeling was performed using Molecular Pro Software. (C) Effect of carbonate content on bicalutamide loading and (D) Effect of carbonate content on CMC. Reproduced with permission from Danquah M, Fujiwara T, Mahato RI. Self-assembling methoxypoly(ethylene glycol)-b-poly(carbonate-co-L-lactide) block copolymers for drug delivery. Biomaterials. 2010;31:2358-70. 
$40 \%$ for PEG-b-PBC to $100 \%$ for poly(ethylene glycol)-block-poly(2-methyl-2-carboxylpropylene carbonate-graft-dodecanol) (PEG-PCD) lipopolymer at 5\% theoretical loading (Figure 2-8A and $\mathbf{C}$ ). This observed increase in drug loading is partly due to structural similarity between embelin and the lipid modified hydrophobic core of PEG-PCD copolymer. Furthermore, it has been shown that polymer-drug compatibility heavily depends on the three-dimensional arrangement and conformation of the different moieties composing the structure of polymer and drug. Mahmud and coworkers have also reported similar results for increasing loading of cucurbitacin I by attaching cholesterol to the poly (caprolactone) of poly(ethylene glycol)- $b$-poly(caprolactone) copolymer. Although the underlying assumptions governing $\chi_{\mathrm{FH}}$ are not entirely applicable to micelles, the trend of its value still provides good indication of drug-core compatibility and can be a logical justification to match a drug to a specific polymer or tailor a new one for it.

Another important feature of PEG-PCD lipopolymer is its potential to enhance both thermodynamic and kinetic stability. When compared to conventional PEG-lipid conjugates, PEG-PCD lipopolymer carries multiple dodecanol lipid chains grafted onto the polycarbonate backbone (Figure 2-8B). Hence, PEG-PCD lipopolymer is capable of forming physical entanglement based cross-linked structures which can enhance hydrophobic interaction in the hydrophobic core. Our studies show PEG-PCD lipopolymer to have a lower $\mathrm{CMC}$ value $\left(\sim 10^{-8} \mathrm{M}\right)$ compared to traditional PEG-lipid conjugates and is expected to demonstrate better in vivo stability.

\section{Length of core forming block}

The length of the hydrophobic core is an important factor which determines the cargo space available for a drug. For a constant hydrophilic block length, an increase in hydrophobic core length typically results in higher drug loading density [172, 173]. This is made possible partly due to a reduction in the pressure-volume work needed to put a drug molecule into the micelle resulting from a larger hydrophobic cargo space. For instance, we have shown that increasing PCD block molecular weights of $1.5 \mathrm{k}, 4 \mathrm{k}$ and 7.25k of PEG-b-PCD copolymer correspondingly increased micelle size from $25 \mathrm{~nm}$ to 42.6 to $129 \mathrm{~nm}$, respectively.

\section{Conclusions and Future Perspectives}

Experimental evidence suggests that the barrier posed by tumor vasculature can potentially be overcome thereby enhancing the extravasation of polymeric nanomedicines to tumors. With advances made in targeting tumor blood vessels and lowering IFP, research focus should be placed on elucidating mechanisms responsible for barrier formation. Future studies should also be centered on "tailor-made" polymeric nanomedicines highly efficient and effective in circumventing the vascular barrier. In particular, thought should be given to site-specific multicompartment polymeric nanomedicines. Such systems will be composed of high binding affinity ligands specific for overexpressed proteins in tumor blood vessels and possess at the minimum a 

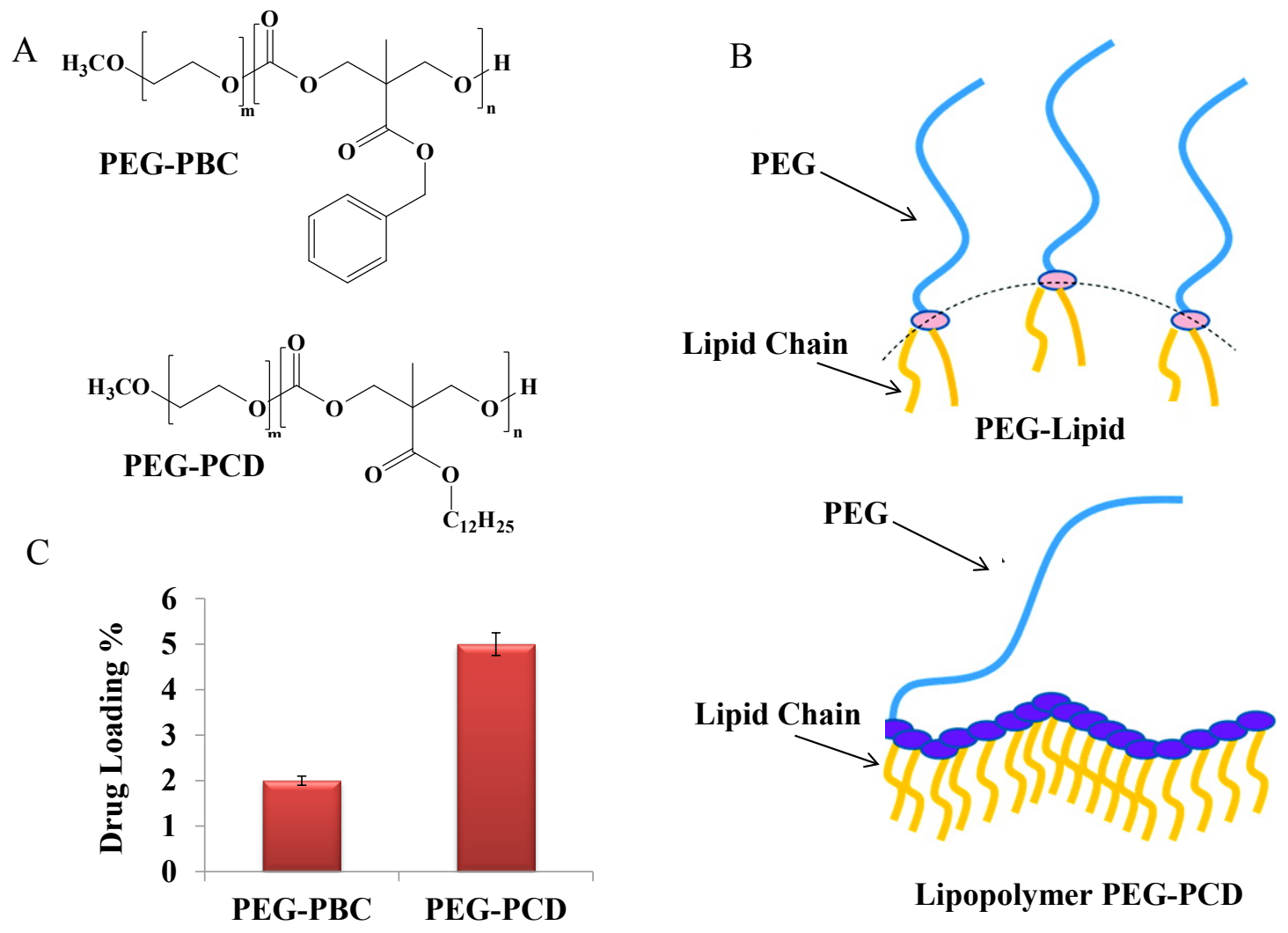

Lipopolymer PEG-PCD

Figure 2-8. Influence of lipid substituent group on polymer architecture and embelin loading.

(A) Chemical structure of poly (ethylene glycol)-b-poly (carbonate) (PEG-PBC) and poly(ethylene glycol)-block-poly(2-methyl-2-carboxyl-propylene carbonate-graftdodecanol) (PEG-PCD). (B) Schematic of architectural structure of PEG-lipid and PEGPCD lipolymer and (C) effect of dodecanol grafted carbonate hydrophobic core on embelin loading. Reproduced with permission from Li F, Danquah M, Mahato RI. Synthesis and characterization of amphiphilic lipopolymers for micellar drug delivery. Biomacromolecules. 2010;11:2610-20. 
bicompartmental core containing agents capable of lowering IFP or altering tumor vasculature as well as potent anticancer agents. Time invested in refining the components of these systems will result in design rules which will facilitate customization of polymeric nanomedicines with phenomenal extravasation for various cancers. Finally, another key challenge which needs to be addressed is improving drug delivery to the avascular core. Logical steps will be to find ways that can re-establish the vascular network in the core of the tumor and improve extravasation at these sites. 


\section{CHAPTER 3. MICELLAR DELIVERY OF BICALUTAMIDE AND EMBELIN FOR TREATING PROSTATE CANCER*}

\section{Introduction}

Prostate cancer is the most pervasive malignancy diagnosed in men and remains the second leading cause of cancer-related mortality affecting men in the United States [174]. The progression of prostate cancer has been found to be androgen-dependent with androgen playing a key role in the proliferation, differentiation, and survival of prostate cancer cells [175-178]. Consequently, androgen ablation, especially the use of antiandrogens, has been used as a standard treatment for men with prostate cancer. Antiandrogens may be divided into two classes: steroidal and nonsteroidal. Steroidal antiandrogens, including cyproterone acetate, interfere with androgen receptor (AR) binding, blocks $5 \alpha$-reductase, and have progesterone-like antigonadotropic activity. The clinical potency of cyproterone has been limited due to loss of erectile potency, influence on carbohydrate metabolism, and associated cardiovascular and hepatocellular toxicity [179, 180].

Nonsteroidal antiandrogens have been developed to avoid the side effects associated with steroidal antiandrogen therapy. Three compounds, namely: flutamide $\left(\right.$ Eulexin $\left.^{\mathrm{TM}}\right)$, nilutamide and bicalutamide (Casodex $\left.{ }^{\mathrm{TM}}\right)$, are available for clinical use. Among them, bicalutamide is the most widely used in androgen ablation therapy due to its relatively long half-life and tolerable side effects [181]. The initial treatment of prostate cancer with bicalutamide yields response in up to $85 \%$ of patients. However, it is not curative, since prolonged treatment results in mutations in the AR, which converts bicalutamide from an antagonist into an agonist leading to drug resistance and the occurrence of hormone-refractory prostate cancer (HRPC).

Resistance to apoptosis is a common feature associated with the progression of prostate cancer from androgen-dependence to HRPC. This defect in the apoptotic machinery prevents neoplastic cells from being naturally eliminated due to the downregulation of proapoptotic and overexpression of antiapoptotic proteins, resulting in an imbalance between the rates of proliferation and apoptosis. Hence, regulators of apoptosis have emerged as attractive targets in the development of therapeutic strategies for treating prostate cancer. Among the key regulators of apoptosis is the inhibitor of apoptosis (IAP) family which suppress caspase activity endogenously preventing cell death [182-184]. The most thoroughly characterized and most potent IAP protein is the X-chromosome-linked IAP (XIAP) [184]. XIAP protein binds and inhibits the initiator caspase- 9 and effector caspases-3 and -7 through the binding of its BIR3 domain and the linker region between BIR1 and BIR2, respectively; consequently inhibiting both

\footnotetext{
* Danquah M, Li F, Duke CB, 3rd, Miller DD, Mahato RI. Micellar Delivery of Bicalutamide and Embelin for Treating Prostate Cancer. Pharmaceutical Research. 2009;26:2081-92.
} 
intrinsic and extrinsic apoptotic pathways [185-188]. Recently, expression levels of XIAP in human prostate cancer cells was found to correlate with apoptotic resistance [189, 190].

Embelin is a novel cell permeable small molecule inhibitor of XIAP, which was discovered by structure-based computational screening of a three-dimensional structure library of natural products derived from traditional Chinese medicine [191]. Embelin binds to the XIAP BIR3 domain preventing its binding to and inhibition of caspase-9 and effector caspases-3 and -7 [191]. Also, embelin has been shown to possess antitumor and anti-inflammatory properties, as well as decrease testosterone levels [192, 193]. Recently, embelin was shown to block nuclear factor- $\mathrm{\kappa B}(\mathrm{NF}-\mathrm{kB})$ signaling pathway resulting in the suppression of NF- $\mathrm{kB}$-regulated antiapoptotic and metastatic gene products, making it a potential effective suppressor of tumor cell survival, proliferation, angiogenesis, invasion, and inflammation [194].

Presently, there are limited treatment options for HRPC and a number of therapeutic strategies including combination therapy are the subject of intense research. Several studies have examined the effect of combining radiation, chemotherapeutic, and hormonal agents in treating prostate cancer. Traditionally, the exploration of combination therapy for treating hormone-refractory prostate cancer involves clinically combining well known cytotoxic chemotherapeutic agents (e.g., docetaxel and paclitaxel) and testing their potency in man. While these studies have shown some drug combinations to have synergistic effect in treating prostate cancer, the advantage may be marginal since their toxicity might exceed their benefit. A current approach in combination therapy requires a paradigm shift, in which the drug regimen incorporates chemotherapeutic agents which are selectively toxic to cancer cells. Therefore, one of our goals was to identify and explore a new combination of therapeutic agents for treating HRPC which is simultaneously potent and less toxic to humans; and can potentially be used for clinical treatment. Since embelin is a powerful inhibitor of XIAP and has minimal effects on normal human prostate epithelial cells [191], we investigated its cytotoxic effect as a single-agent and possible synergism in combination with bicalutamide in two androgen receptor containing human prostate cancer cell lines: $\mathrm{LNCaP}$, which is androgen dependent and C4-2, which is androgen independent. The structures of bicalutamide and embelin are shown in Figure 3-1A. We hypothesize that therapeutic combinations of bicalutamide and embelin will have a synergistic effect on growth inhibition and apoptosis of $\mathrm{LNCaP}$ and $\mathrm{C} 4-2$ cells as well as tumor regression in xenograft mouse models considering their mechanism of action (Figure 3-1B).

Polymeric micelles are known to improve the solubility, stability, site specificity and hence therapeutic efficacy of hydrophobic drugs. These micelles self-assemble into nanosized $(20-60 \mathrm{~nm})$, spherical structures with a hydrophobic core capable of solubilizing a considerable amount of highly water insoluble drugs. The stealth properties associated with poly(ethylene oxide) (PEO) hydrophilic corona of polymeric micelles prevents recognition by the reticuloendothelial system (RES) and therefore minimizes elimination of the micelles from the bloodstream [156]. Thus, these so called 'stealth' properties of the PEO shell result in increased blood circulation times and prevention of 
A<smiles>C[C@](O)(CNc1ccc(F)cc1)C(=O)Nc1ccc(C#N)c(C(F)(F)F)c1</smiles>

Bicalutamide<smiles>CCCCCCCCCCCC1=C(O)C(=O)C=C(O)C1=O</smiles>

Embelin

B

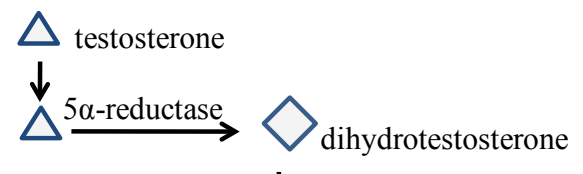

Androgen-sensitive cell
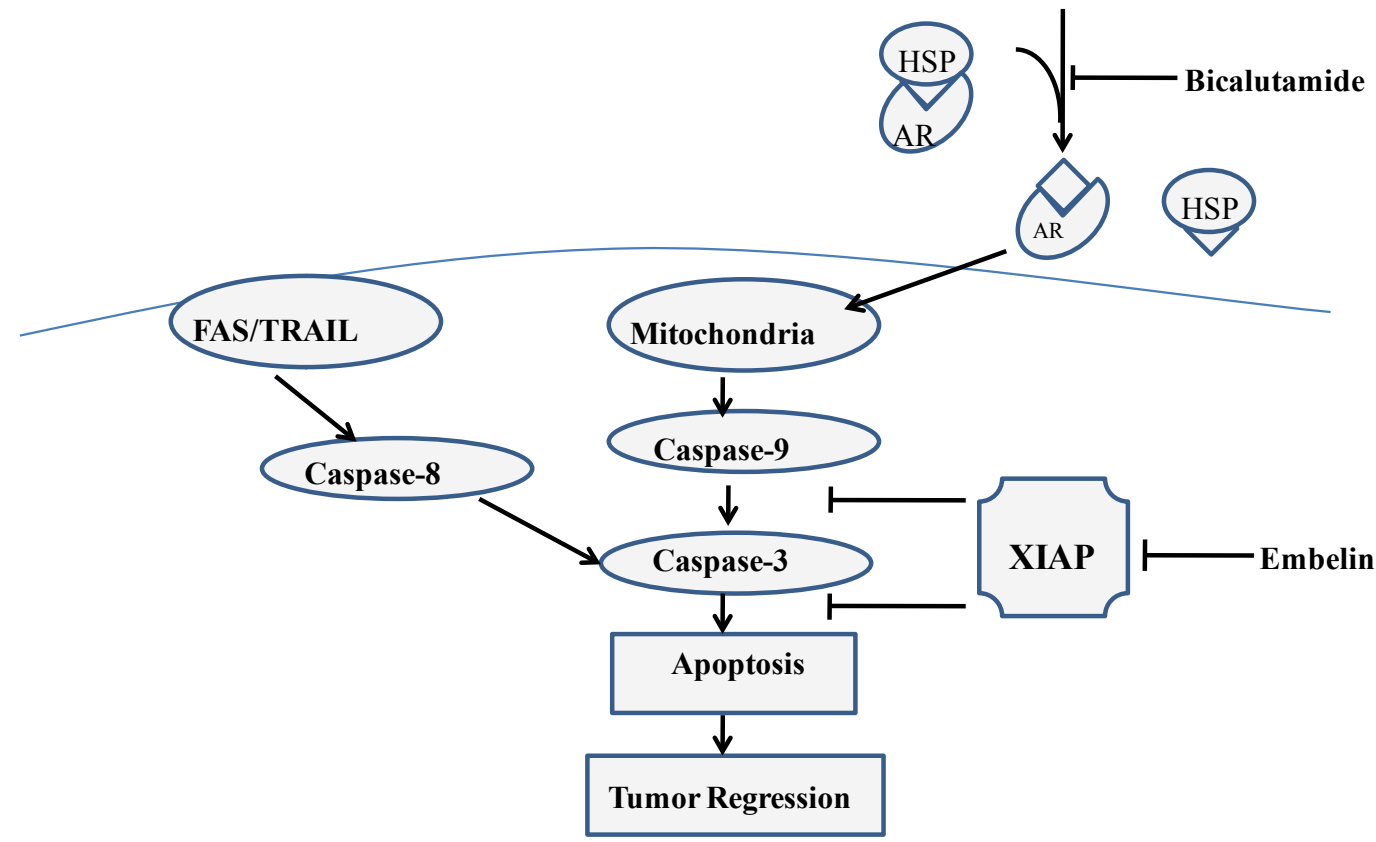

Figure 3-1. Therapeutic strategy for bicalutamide and embelin based combination therapy.

(A) Chemical structures of bicalutamide and embelin and (B) schematic diagram showing the combined effect of bicalutamide and embelin on apoptosis in androgen-sensitive cells and on tumor regression. 
plasma protein binding [157]. In addition, the small size ensures preferential accumulation in tumor cells via the enhanced permeability and retention (EPR) effect $[158,195]$.

In this study, polymeric micelles were fabricated using poly(ethylene glycol)-bpoly(lactic acid) [PEG-PLA] copolymer and used for enhancing the water solubility and bioavailability of bicalutamide and embelin to improve their efficacy. The enhanced drug water solubility and bioavailability translate into a reduction in the administered dose and possible toxicity in normal cells. In the current study, PLA was selected as the hydrophobic core-forming block since it is Food and Drug Administration (FDA) approved for clinical use and hence may facilitate the translation of our therapeutic agents for clinical applications. In addition, the in vitro synergistic or additive antiproliferative effects of combined bicalutamide and embelin, each possessing distinct cytotoxic mechanisms, was studied in both androgen dependent (LNCaP) and androgen independent (C4-2) human prostate cancer cells, while mechanistic studies on the induction of apoptotic cell death by bicalutamide was also conducted. Finally, the ability of sequential exposure to bicalutamide-loaded micelles followed by embelinloaded micelles to regress prostate cancer tumors in xenograft mice models was examined.

\section{Materials and Methods}

\section{Materials}

PEG (5100)-b-PLA (4500) copolymer $(\mathrm{Mn}=9600)$ was purchased from Polymer Source (Montreal, Canada). Embelin was purchased from Sigma-Aldrich (St. Louis, MO) while bicalutamide was synthesized as described by Mukherjee et al. [196] RPMI 1640 medium was obtained from Invitrogen (Carlsbad, CA) and human prostate cancer cells C4-2 and lymph node prostate adenocarcinoma (LNCaP) were purchased from American Type Culture Collection (ATCC, Manassas, VA). Caspase-Glo ${ }^{\mathrm{TM}}$ 3/7, 8 and 9 assay and RNA extraction kits were purchased from Promega (Madison, WI). SYBR Green real time PCR master mix and reverse transcription reagents were purchased from Applied Biosystems (Foster city, CA). Human Total XIAP ELISA kit was purchased from R\&D Systems (Minneapolis, MN). All other reagents were obtained from Sigma-Aldrich (St. Louis, MO) unless otherwise stated and were used as received.

In vitro cell viability assays

Human prostate cancer cells, LNCaP and C4-2 cells (American Type Culture Collection) were incubated in RPMI 1640 media supplemented with $2 \mathrm{mM}$ L-glutamine, $10 \%$ fetal bovine serum and $1 \%$ penicillin-streptomycin at $37^{\circ} \mathrm{C}$ in humidified environment of 5\% $\mathrm{CO}_{2}$ and subcultured every 3-4 days to maintain exponential growth. 
Cells were seeded in 96-well plates at a density of $5 \times 10^{3}$ viable cells/well and incubated for $48 \mathrm{~h}$ to permit cell attachment. The cells were exposed to bicalutamide or embelin at concentrations ranging from 1 to $100 \mu \mathrm{M}$ for $24 \mathrm{~h}$. At the end of treatment, 20 $\mu \mathrm{l}$ of MTT $(5 \mathrm{mg} / \mathrm{ml})$ was added to each well and incubated for 3-4 $\mathrm{h}$. The plates were then centrifuged at $1500 \mathrm{x}$ g for $2 \mathrm{~min}$ and the medium aspirated. The residual formazan crystals were solubilized with $100 \mu \mathrm{DMSO}$ and the plate analyzed using a microplate reader recording absorbance values at a test wavelength of $560 \mathrm{~nm}$. Cell viability for a given concentration was expressed as a percentage of the intensity of controls.

Caspase detection

Caspase-Glo 3/7, 8 and 9 assay kits purchased from Promega (Madison, WI)) were used to analyze caspase 3, 8 and 9 activities, respectively, as per the manufacturer's protocol. Briefly, $100 \mu \mathrm{l}$ Caspase-Glo reagent was added to $100 \mu \mathrm{l}$ of culture supernatants in 96-well plates and incubated at room temperature for $1 \mathrm{~h}$. The contents were then transferred into disposable culture tubes and luminescence was determined using a Berthold Detection Systems Sirius luminometer (Pforzheim, Germany).

\section{XIAP expression analysis}

The mRNA and protein level expression of XIAP in LNCaP and C4-2 cells after treatment with embelin for $48 \mathrm{~h}$ was assayed using quantitative real-time PCR and ELISA, respectively. Briefly, total RNA was isolated using RNAeasy mini isolation kit from Promega. RNA concentration was measured by UV spectrophotometry with a Biomate 3 spectrophotometer. One hundred and fifty nanograms (ng) of extracted RNA was converted into cDNA using Multi-Scribe reverse transcriptase and random hexamers (Applied Biosystems, Inc., Foster City, CA) by incubation at $25^{\circ} \mathrm{C}$ for $10 \mathrm{~min}$, followed by reverse transcription at $48^{\circ} \mathrm{C}$ for $30 \mathrm{~min}$ and enzyme inactivation at $95^{\circ} \mathrm{C}$ for $5 \mathrm{~min}$. To determine the level of XIAP expression, the following XIAP-specific primers were used: Forward: 5'-TGT TTC AGC ATC AAC ACT GGC ACG-3'; Reverse: 5'-TGC ATG ACA ACT AAA GCA CCG GAC-3' (NM_001167). The PCR conditions included denaturation at $95^{\circ} \mathrm{C}$ for $10 \mathrm{~min}$, followed by 40 cycles of amplification by sequential denaturation at $95^{\circ} \mathrm{C}$ for $15 \mathrm{~s}$ and primer annealing as well as strand extension for $1 \mathrm{~min}$. XIAP gene expression was normalized to $\beta$-actin as internal control. To confirm the amplification specificity, the PCR products were subjected to melting curve analysis. The expressed XIAP levels were quantified and normalized to the total amount of cDNA used.

For XIAP protein expression, total protein was isolated by lysing cells. XIAP levels were measured as per the protocol of Human Total XIAP ELISA kit obtained from R\&D Systems (Minneapolis, MN). Results were normalized by measuring the total protein using a BCA assay kit. 
Isobologram analysis

The dose-response interaction between bicalutamide and embelin at the point of $\mathrm{IC}_{50}$ was assessed to be synergistic, additive or antagonistic using the isobologram method of analysis of Steel and Peckham [197]. Dose-response curves were plotted for the effects of bicalutamide and embelin on human prostate cancer LNCaP and C4-2 cell viability. From these curves, the combined drug $\mathrm{IC}_{50}$ values were determined for each curve. Specifically, LNCaP and C4-2 cells were treated with embelin $(0,5,10,25$ and 50 $\mu \mathrm{M})$ and bicalutamide $(0,10,25$ and $50 \mu \mathrm{M})$ either simultaneously or sequentially and cell viability assessed using MTT assay resulting in the above-mentioned dose-response curves. The combination index (CI) was calculated by Equation 3-1:

$$
\mathrm{CI}=\frac{\mathrm{d} 1}{\mathrm{D}_{50} 1}+\frac{\mathrm{d} 2}{\mathrm{D}_{50} 2}
$$

where $\mathrm{D}_{50} 1$ is the dose of agent 1 (bicalutamide) required to produce 50 percentage effect alone, and $\mathrm{d} 1$ is the dose of agent 1 required to produce the same 50 percentage effect in combination with $\mathrm{d} 2$. Similarly, $\mathrm{D}_{50} 2$ is the dose of agent 2 (embelin) required to produce 50 percentage effect alone, and $\mathrm{d} 2$ is the dose of agent 2 required to produce the same 50 percentage effect in combination with $\mathrm{d} 1$. The CI values were interpreted as follows: $<1.0$, synergism; 1.0, additive effect; $>1.0$, antagonism. Each experiment was performed three times. The parameters $\mathrm{d} 1$ and $\mathrm{d} 2$ in equation 1 were obtained as follows: when a dose of bicalutamide (d1) was selected the incremental effect produced by adding embelin starting from 0 to $50 \mu \mathrm{M}$ was assessed. The concentration of embelin that when combined with $\mathrm{d} 1$ resulted in the inhibition of $50 \%$ cell growth was designated $\mathrm{d} 2$.

Fabrication of drug-loaded micelles

The film sonication method was used to load bicalutamide and embelin into the core of polyethylene glycol-polylactic acid (PEG-PLA) micelles. All experiments were performed using a theoretical loading of 5\% unless otherwise stated. Briefly, $1 \mathrm{mg}$ of bicalutamide or embelin and $19 \mathrm{mg}$ of PEG-PLA was dissolved in $5 \mathrm{ml}$ methanol. The mixture was allowed to stir for $5 \mathrm{~min}$ and the solvent evaporated. The resulting film was hydrated and sonicated for 7 min using a Misonix ultrasonic liquid processor (Farmingdale, NY) with an output power of $25 \mathrm{~W}$. The resultant formulation was then centrifuged at 5,000 rpm for 10 min to separate micelles from residual free drug. Subsequently, the supernatant was filtered using a $0.22 \mu \mathrm{m}$ nylon filter. The micelle preparation was immediately lyophilized for two days and stored at $4{ }^{\circ} \mathrm{C}$ to prolong shelflife and avoid untimely release of the drug.

Critical micelle concentration

Fluorescence spectroscopy was used to estimate the critical micelle concentration $(\mathrm{CMC})$ of PEG-PLA copolymer using pyrene as a hydrophobic fluorescent probe. Nine 
samples of PEG-PLA dissolved in methanol with concentrations ranging from $1 \times 10^{-8}$ to $1 \mathrm{~g} / \mathrm{L}$ were prepared and allowed to equilibrate with a constant pyrene concentration of 6 $\mathrm{x} 10^{-7} \mathrm{M}$ for $48 \mathrm{~h}$ at room temperature. The fluorescence spectra of pyrene were recorded with a Molecular Devices SpectraMax M2/M2 ${ }^{\mathrm{e}}$ spectrofluorometer (Sunnyvale, CA). An excitation wavelength of $390 \mathrm{~nm}$ was used and the emission spectra recorded from 320 to $450 \mathrm{~nm}$ with both bandwidths set at $2 \mathrm{~nm}$. Peak height intensity ratio $\left(\mathrm{I}_{3} / \mathrm{I}_{1}\right)$ of the third peak $\left(\mathrm{I}_{3}\right.$ at $\left.338 \mathrm{~nm}\right)$ to the first peak $\left(\mathrm{I}_{1}\right.$ at $333 \mathrm{~nm}$ ) was plotted against the logarithm of polymer concentration. The value of the $\mathrm{CMC}$ was obtained as the point of intersection of two tangents drawn to the curve at high and low concentrations, respectively.

Drug loading density and encapsulation efficiency

Drug loading was determined as follows: Lyophilized drug-loaded micelles were dissolved in methanol and the drug present in solution measured by ultraviolet spectroscopy. The weight of drug loaded in the micelles was calculated using a calibration curve. Background absorbance interference from PEG-PLA copolymer was accounted for by measuring the absorbance of blank PEG-PLA micelles under the same conditions. The micelle drug loading content and encapsulation efficiency were obtained by Equations 3-2 and 3-3.

$$
\begin{gathered}
\text { drug loading density }=\frac{\text { weight of drug in micelle }}{\text { weight of micelle }} \times 100 \% \\
\text { drug encapsulation efficiency }=\frac{\text { weight of drug in micelle }}{\text { weight of drug originally fed }} \times 100 \%
\end{gathered}
$$

Size and size distribution

Mean particle size and size distribution of drug-loaded micelles were measured via dynamic light scattering using a Malvern instruments Zetasizer Nano Series (Worcestershire, United Kingdom). Samples were diluted to appropriate concentrations and analyzed at room temperature with a $90^{\circ}$ detection angle. Mean micelle size was obtained as a Z-average which is an intensity mean. All measurements were repeated 7 times and reported as the mean diameter \pm SD for triplicate samples.

Solubility studies of drug-loaded micelles

The water solubility of free drug (bicalutamide or embelin) and drug-loaded micelles was determined by shaking an excess amount of free drug and $3 \mathrm{mg}$ of drugloaded micelles in $1 \mathrm{~mL}$ water, respectively. The suspension was then centrifuged at 8000 $\mathrm{x} g$ for $10 \mathrm{~min}$ and filtered on $0.2 \mu \mathrm{m}$ cellulose membrane. The amount of bicalutamide in the saturated solution was evaluated by UV spectroscopy as described above. 
In vivo efficacy assessment of bicalutamide-loaded micelles in xenografts

All animal experiments were performed in accordance with NIH animal use guidelines and the protocol approved by the Animal Care and Use Committee at the University of Tennessee Health Science Center. Xenograft flank tumors were induced in 8 week old male BALB/C nude mice purchased from The Jackson Laboratory (Bar Harbor, ME) by subcutaneous injection of 3 million LNCaP cells suspended in 1:1 media and matrigel. When tumors reached approximately $150 \mathrm{~mm}^{3}$, mice were randomized into three groups of 5 mice, minimizing weight and tumor size differences. Each group was treated with intratumoral injection of saline, sonicated bicalutamide suspension or bicalutamide-loaded micelles $(20 \mathrm{mg} / \mathrm{kg})$ three times a week. Tumors were measured with a caliper prior to each injection, and their volumes calculated using the formula: (width ${ }^{2} \mathrm{x}$ length) $/ 2$.

\section{Results}

Effect of bicalutamide on apoptosis

To determine whether prostate cancer cell death by bicalutamide is also associated with apoptosis, we determined the activation of initiator caspases 8 and 9, and effector caspase 3 at $48 \mathrm{~h}$ post-incubation of LNCaP cells with bicalutamide using caspase luminescence assay. Results were normalized by measuring the total protein using BCA protein assay kit. As shown in Figure 3-2, relatively high levels of caspase 3 and 9 compared to caspase 8 were observed at the drug concentrations of 50 and 100 $\mu \mathrm{g} / \mathrm{ml}$.

Effect of embelin on caspase activation and XIAP inhibiton

Resistance to apoptosis is a characteristic feature of prostate cancer and XIAP is known to inhibit the activation of the initiator caspase 9 and the effector caspase 3 which is the committed step in the apoptotic pathway. Hence, we determined the levels of caspase activation and XIAP expression levels in LNCaP and C4-2 cells after treatment with $5 \mu \mathrm{M}$ embelin (XIAP inhibitor) for $48 \mathrm{~h}$. As demonstrated in Figure 3-3A, caspase activation was higher in the embelin treated cells. Specifically, caspase 8 had lower activation levels compared to caspases 3 and 9, with caspase 9 activity being the highest. However, the caspase 3 and 9 activation was found to be lower in C4-2 cells compared to LNCaP cells. To determine whether the differential levels of caspase activity in LNCaP and C4-2 cells correlate with XIAP expression, we measured baseline XIAP expression in both cells and the effect of embelin in inhibiting XIAP expression using ELISA and real time RT-PCR, respectively (Figure 3-3B). The data show that C4-2 cells have higher baseline XIAP expression levels than LNCaP, and treatment with $5 \mu \mathrm{M}$ embelin resulted in a decrease in XIAP expression in both cell lines. However, the decrease in XIAP 


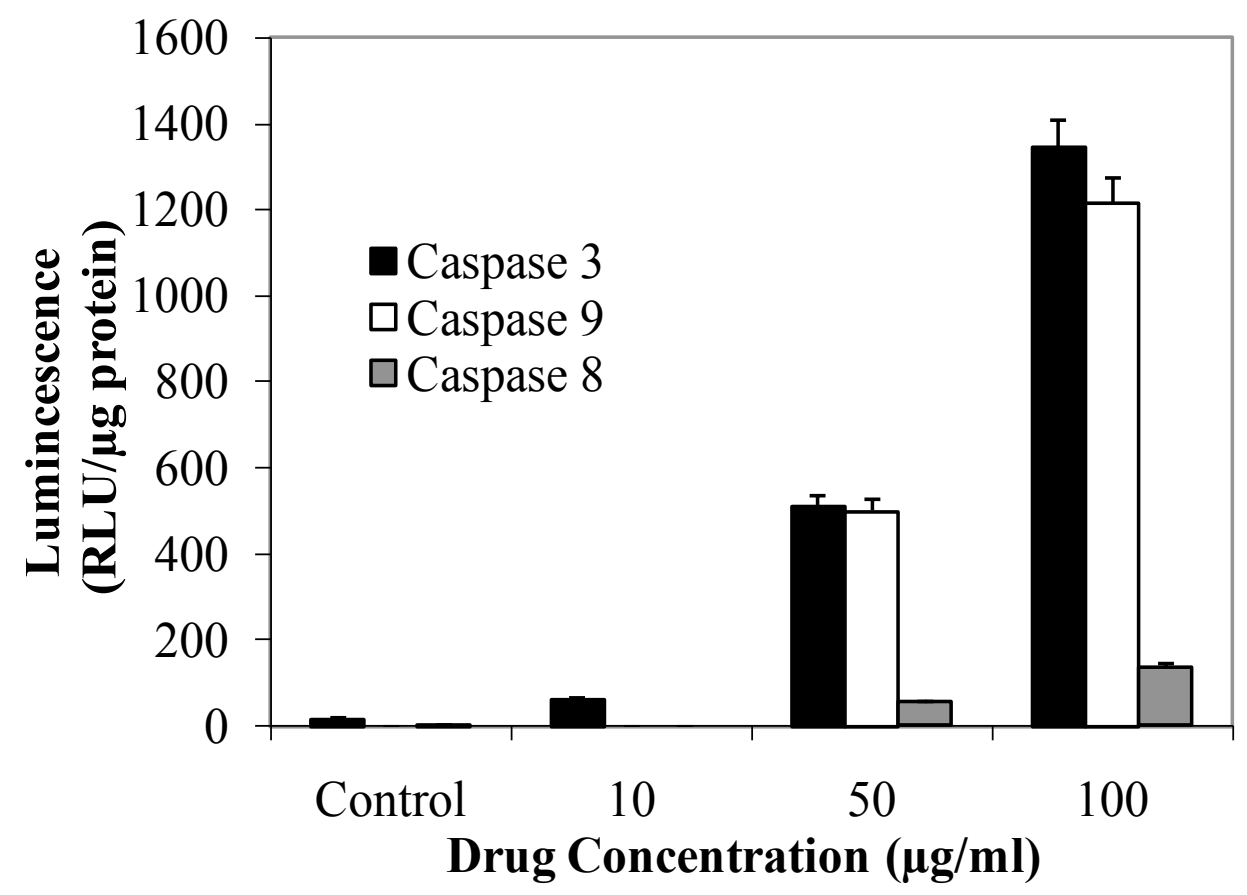

Figure 3-2. Effect of bicalutamide on the activation of caspases in LNCaP cells.

At $48 \mathrm{~h}$ postincubation with the drug at a dose of 0 (control), 10, 50 and $100 \mu \mathrm{g} / \mathrm{ml}$, cells were lyzed to determine caspase 3,8 and 9 activities in terms of relative light units (RLU) and total protein using a BCA protein assay kit. Results are presented as the mean \pm S.D. of triplicates. 
A

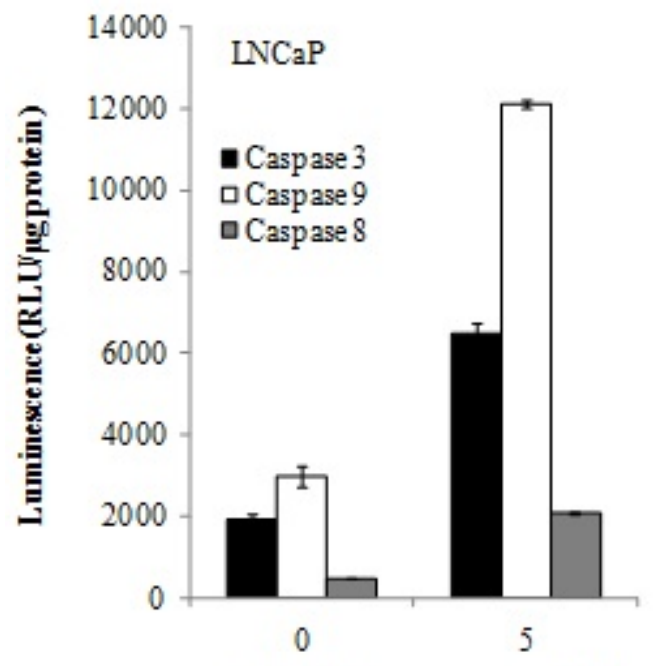

Embelin Concentration $(\mu \mathrm{M})$
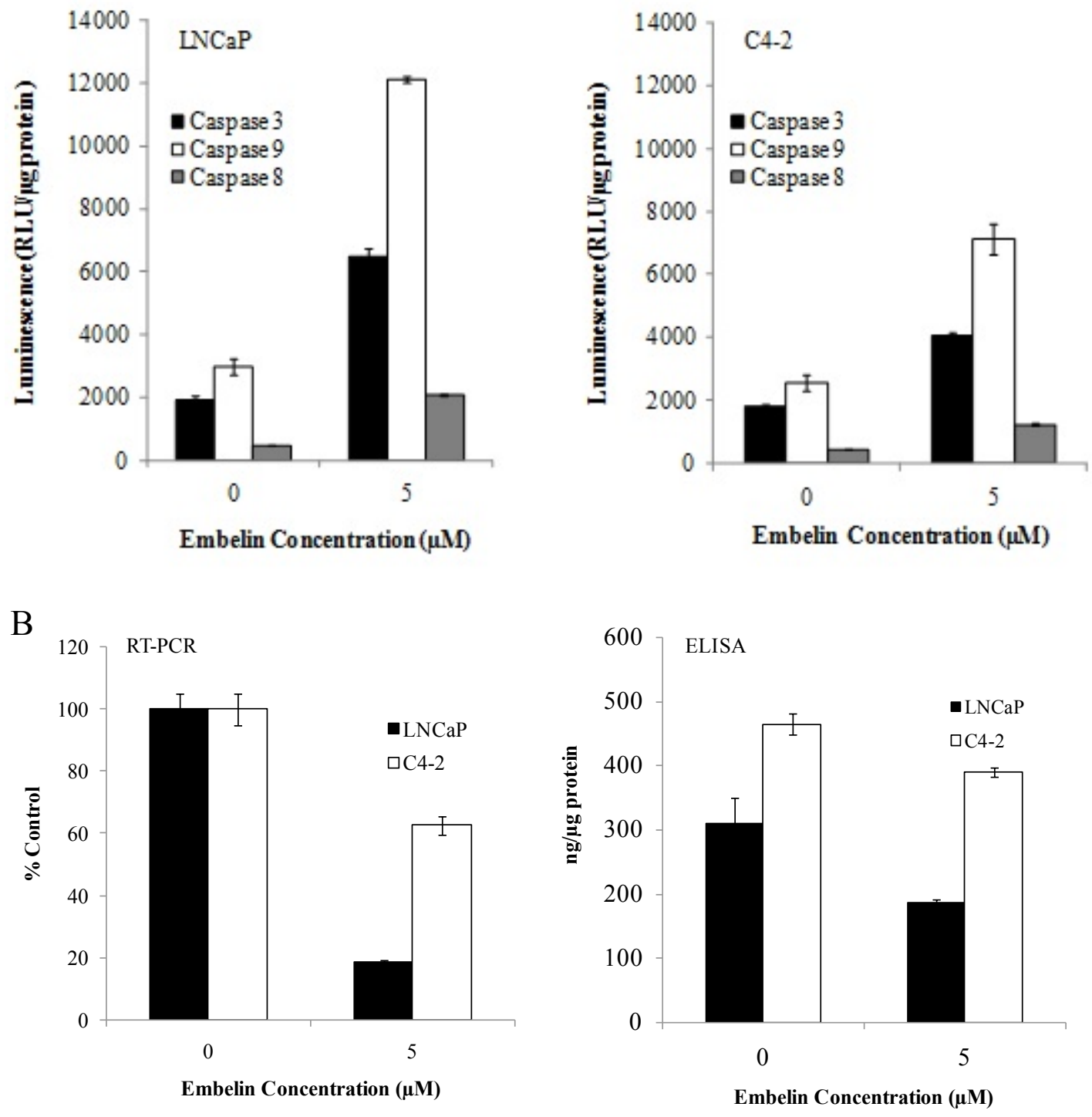

Figure 3-3. Effect of Embelin on caspase activation and XIAP inhibition in LNCaP and C4-2 prostate cancer cells.

(A) Embelin effectively activates caspases 3, 8 and 9 in LNCaP and C4-2 cells. Activation of caspases is higher in LNCaP cells which have comparatively lower XIAP expression. Results are presented as the mean \pm S.D. of triplicates. (B) XIAP expression in LNCaP and C4-2 cells using RT-PCR and ELISA. 
expression upon treatment with embelin was more pronounced in LNCaP cells compared to $\mathrm{C} 4-2$ cells.

Effect of bicalutamide and embelin on prostate cancer cell growth

To determine whether embelin can be used for treating androgen dependent and hormone refractory prostate cancer, we determined the $\mathrm{IC}_{50}$ values of both bicalutamide and embelin in two androgen receptor containing human prostate cancer cells, androgen sensitive LNCaP and hormone refractory C4-2 prostate cancer cells. As shown in Figure 3-4, embelin was more potent than bicalutamide in killing prostate tumor cells irrespective of their androgen status. LNCaP cells were more sensitive to bicalutamide compared to $\mathrm{C} 4-2$ cells, with a $50 \%$ inhibitory concentration $\left(\mathrm{IC}_{50}\right)$ of approximately 43 and $93 \mu \mathrm{M}$, respectively (Figure 3-4A). Hence, C4-2 cells which are androgen independent and AR positive displayed a two-fold resistance to bicalutamide compared to LNCaP cells which are androgen dependent. In contrast, embelin exhibited superior antiproliferative activity in $\mathrm{LNCaP}$ and $\mathrm{C} 4-2$ cells, with $\mathrm{IC}_{50}$ of $\sim 5 \mu \mathrm{M}$ (Figure 3-4B). However, both cell lines displayed similar sensitivity to embelin at the concentrations examined.

We used the isobologram method of Steel and Peckham [197] to assess whether the simultaneous or sequential combination of these two drugs could confer synergistic, additive, or antagonistic effects. Combination index (CI) was determined after treating 1 x $10^{4} \mathrm{C} 4-2$ cells with a combination of bicalutamide and embelin. Cell viability was determined by MTT assay and the resulting dose-response curves are shown in Figure 3-5. CI was calculated by the formula:CI $=\left(\mathrm{d} 1 / \mathrm{D}_{50} 1\right)+\left(\mathrm{d} 2 / \mathrm{D}_{50} 2\right)$, where $\mathrm{D}_{50} 1$ is the dose of bicalutamide required to produce $50 \%$ effect alone, and $\mathrm{d} 1$ is the dose of bicalutamide required to produce the same $50 \%$ effect in combination with $\mathrm{d} 2 . \mathrm{D}_{50} 2$ is similarly the dose of embelin required to produce $50 \%$ effect alone, and $\mathrm{d} 2$ is the dose of embelin required to produce the same $50 \%$ effect in combination with $\mathrm{d} 1$. The CI values are interpreted as follows: $<1.0$, synergism; 1.0, additive; and $>1.0$, antagonism. The data is reported in Tables 3-1, 3-2 and 3-3. These results suggest that the cytotoxic effect of the combination of bicalutamide and embelin was dependent on the treatment schedule and cell line. When the combination of these two drugs were used, simultaneous exposure and sequential treatment of bicalutamide followed by embelin was synergistic in C4-2 cells, while additive and antagonistic effects were observed for LNCaP cells. Sequential treatment of embelin followed by bicalutamide was additive and antagonistic for both cell lines.

Characterization of polymeric micelles

Micelles were fabricated with polyethylene glycol-b-polylactic acid (PEG-PLA) di-block copolymer using the film sonication method. We determined the critical micelle concentration $(\mathrm{CMC})$ using pyrene as a hydrophobic probe to observe micelle formation 
A

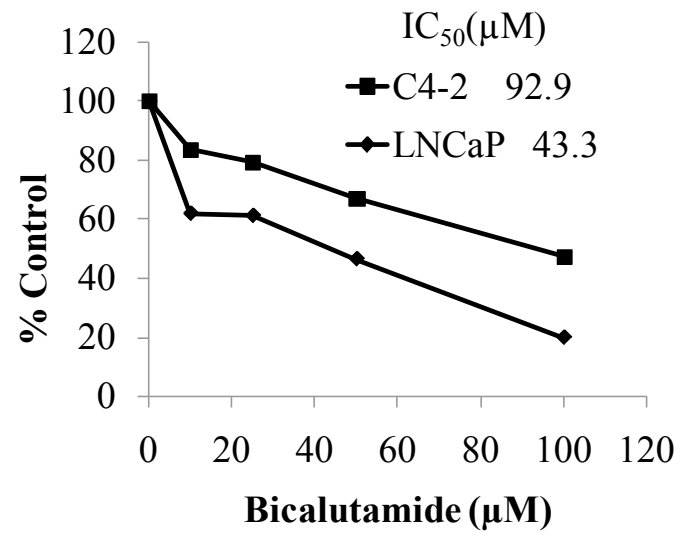

$\mathrm{B}$

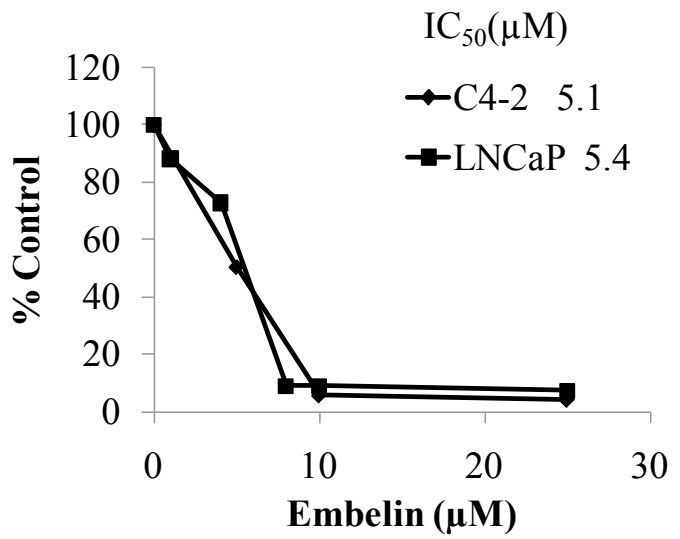

Figure 3-4. Effect of drug treatment on cell viability in prostate cancer cells $(\mathrm{C4} 4-2$ and LNCaP).

(A) C4-2 and LNCaP cells treated with increasing concentrations of bicalutamide (0-100 $\mu \mathrm{M})$ for $24 \mathrm{~h}$. (B) C4-2 and $\mathrm{LNCaP}$ cells treated with increasing concentrations of embelin $(0-25 \mu \mathrm{M})$ for $24 \mathrm{~h}$. Results are expressed as the percentage of control. 

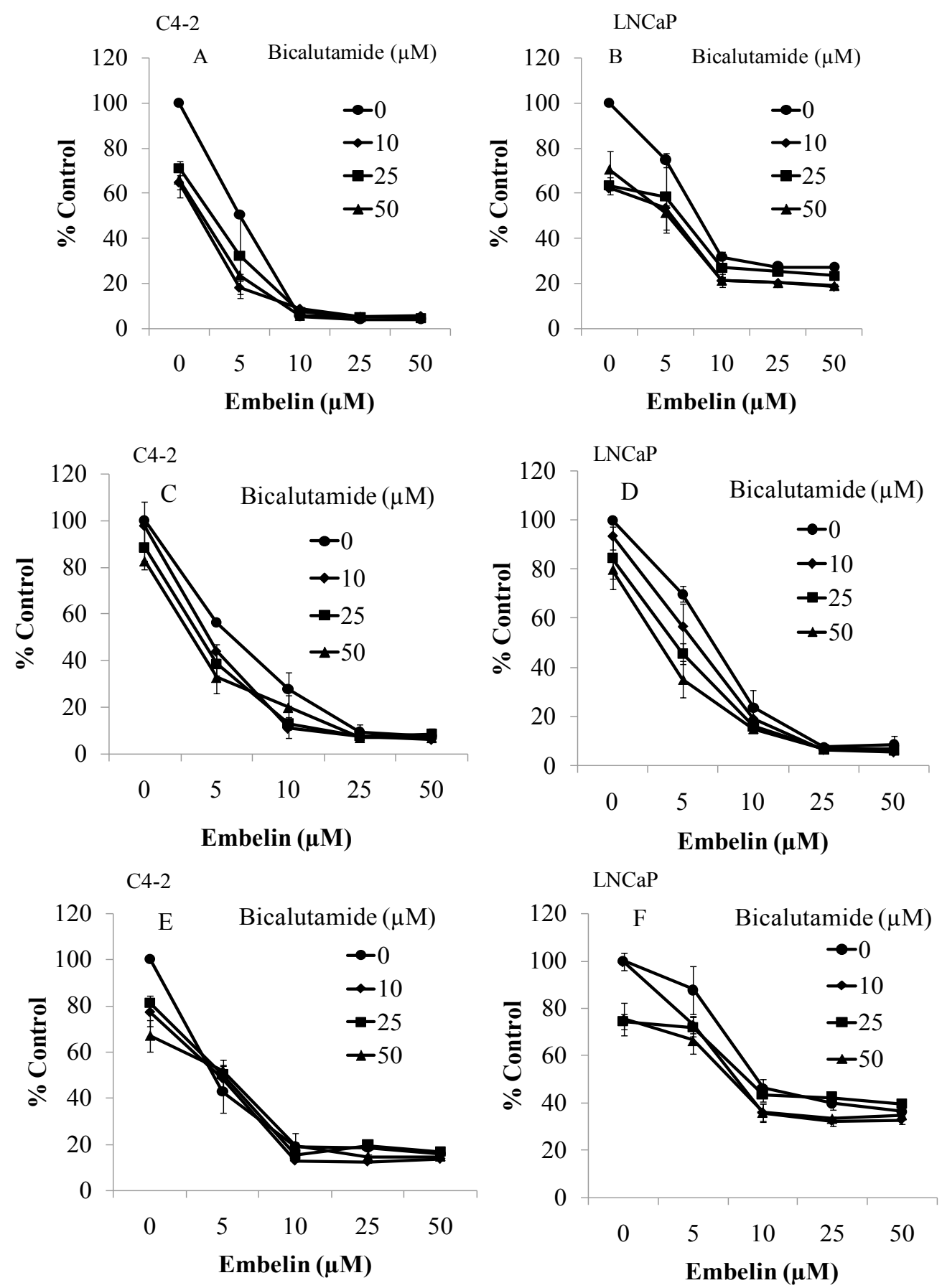

Figure 3-5. Dose-response curves for bicalutamide and embelin combination in human prostate cancer cells.

(A) and (B) Simultaneous exposure to bicalutamide and embelin in C4-2 and LNCaP cells, respectively. (C) and (D) Sequential exposure to bicalutamide followed by embelin in C4-2 and LNCaP cells, respectively. (E) and (F) Sequential exposure to embelin followed by bicalutamide in $\mathrm{C} 4-2$ and $\mathrm{LNCaP}$ cells, respectively. 
Table 3-1. Synergistic antiproliferative activity of bicalutamide $(\mathrm{d} 1=10 \mu \mathrm{M})$ and embelin in human prostate cancer cells.

\begin{tabular}{lllll}
\hline Drug combination & Cell line & $\mathbf{d 1}(\boldsymbol{\mu M})$ & $\mathbf{d 2}(\boldsymbol{\mu M})$ & CI \\
\hline \multirow{2}{*}{ Bicalutamide + Embelin } & & & & \\
& C4-2 & 10 & 1.59 & 0.42 \\
& LNCaP & 10 & 4.66 & 1.09 \\
Bicalutamide $\rightarrow$ Embelin & C4-2 & 10 & 4.51 & 0.87 \\
& LNCaP & 10 & 5.90 & 1.05 \\
Embelin $\rightarrow$ Bicalutamide & C4-2 & 10 & 5.65 & 1.22 \\
& LNCaP & 10 & 8.09 & 1.08 \\
\hline
\end{tabular}

Combination Index (CI) of simultaneous and sequential treatment of bicalutamide and embelin in C4-2 and LNCaP cells. $5 \times 10^{3}$ cells were simultaneously treated with a combination of bicalutamide and embelin or sequentially [(bicalutamide followed by embelin) or (embelin followed by bicalutamide)]. Cell viability was determined by MTT assay. The combination index $(\mathrm{CI})$ was calculated by the formula: $\mathrm{CI}=\left(\mathrm{d} 1 / \mathrm{D}_{50} 1\right)+$ $\left(\mathrm{d} 2 / \mathrm{D}_{50} 2\right)$, where $\mathrm{D}_{50} 1$ is the dose of bicalutamide required to produce $50 \%$ effect alone, and $\mathrm{d} 1$ is the dose of bicalutamide required to produce the same $50 \%$ effect in combination with $\mathrm{d} 2 . \mathrm{D}_{50} 2$ is similarly the dose of embelin required to produce $50 \%$ effect alone, and $\mathrm{d} 2$ is the dose of embelin required to produce the same $50 \%$ effect in combination with $\mathrm{d} 1.10 \mu \mathrm{M}$ bicalutamide was combined with $0,5,10,25$ and $50 \mu \mathrm{M}$ embelin. The CI values are interpreted as follows: $<1.0$, synergism; 1.0 , additive; and $>1.0$, antagonism. Each experiment was done in triplicate. 
Table 3-2. Synergistic antiproliferative activity of bicalutamide $(\mathrm{d} 1=25 \mu \mathrm{M})$ and embelin in human prostate.

\begin{tabular}{lllll}
\hline Drug combination & Cell line & dl $(\boldsymbol{\mu M})$ & d2 $(\boldsymbol{\mu M})$ & CI \\
\hline \multirow{2}{*}{ Bicalutamide + Embelin } & & & & \\
& C4-2 & 25 & 2.74 & 0.81 \\
& LNCaP & 25 & 5.46 & 1.61 \\
Bicalutamide $\rightarrow$ Embelin & C4-2 & 25 & 3.85 & 0.92 \\
& LNCaP & 25 & 4.47 & 1.20 \\
Embelin $\rightarrow$ Bicalutamide & C4-2 & 25 & 5.80 & 1.41 \\
& LNCaP & 25 & 8.88 & 1.51 \\
\hline
\end{tabular}

Combination Index (CI) of simultaneous and sequential treatment of bicalutamide and embelin in C4-2 and LNCaP cells. $5 \times 10^{3}$ cells were simultaneously treated with a combination of bicalutamide and embelin or sequentially [(bicalutamide followed by embelin) or (embelin followed by bicalutamide)]. Cell viability was determined by MTT assay. The combination index $(\mathrm{CI})$ was calculated by the formula: $\mathrm{CI}=\left(\mathrm{d} 1 / \mathrm{D}_{50} 1\right)+$ $\left(\mathrm{d} 2 / \mathrm{D}_{50} 2\right)$, where $\mathrm{D}_{50} 1$ is the dose of bicalutamide required to produce $50 \%$ effect alone, and $\mathrm{d} 1$ is the dose of bicalutamide required to produce the same $50 \%$ effect in combination with $\mathrm{d} 2 . \mathrm{D}_{50} 2$ is similarly the dose of embelin required to produce $50 \%$ effect alone, and $\mathrm{d} 2$ is the dose of embelin required to produce the same $50 \%$ effect in combination with $\mathrm{d} 1.25 \mu \mathrm{M}$ bicalutamide was combined with $0,5,10,25$ and $50 \mu \mathrm{M}$ embelin. The CI values are interpreted as follows: $<1.0$, synergism; 1.0 , additive; and $>1.0$, antagonism. Each experiment was done in triplicate. 
Table 3-3. Synergistic antiproliferative activity of bicalutamide $(\mathrm{d} 1=50 \mu \mathrm{M})$ and embelin in human prostate.

\begin{tabular}{lllll}
\hline Drug combination & Cell line & dl $(\boldsymbol{\mu M})$ & d2 $(\boldsymbol{\mu M})$ & CI \\
\hline \multirow{2}{*}{ Bicalutamide + Embelin } & & & & \\
& C4-2 & 50 & 1.90 & 0.91 \\
& LNCaP & 50 & 4.27 & 1.94 \\
Bicalutamide $\rightarrow$ Embelin & C4-2 & 50 & 3.26 & 1.09 \\
& LNCaP & 50 & 3.42 & 1.63 \\
Embelin $\rightarrow$ Bicalutamide & C4-2 & 50 & 6.33 & 1.78 \\
& LNCaP & 50 & 7.71 & 1.96 \\
\hline
\end{tabular}

Combination Index (CI) of simultaneous and sequential treatment of bicalutamide and embelin in C4-2 and LNCaP cells. $5 \times 10^{3}$ cells were simultaneously treated with a combination of bicalutamide and embelin or sequentially [(bicalutamide followed by embelin) or (embelin followed by bicalutamide)]. Cell viability was determined by MTT assay. The combination index $(\mathrm{CI})$ was calculated by the formula: $\mathrm{CI}=\left(\mathrm{d} 1 / \mathrm{D}_{50} 1\right)+$ $\left(\mathrm{d} 2 / \mathrm{D}_{50} 2\right)$, where $\mathrm{D}_{50} 1$ is the dose of bicalutamide required to produce $50 \%$ effect alone, and $\mathrm{d} 1$ is the dose of bicalutamide required to produce the same $50 \%$ effect in combination with $\mathrm{d} 2 . \mathrm{D}_{50} 2$ is similarly the dose of embelin required to produce $50 \%$ effect alone, and $\mathrm{d} 2$ is the dose of embelin required to produce the same $50 \%$ effect in combination with $\mathrm{d} 1.50 \mu \mathrm{M}$ bicalutamide was combined with $0,5,10,25$ and $50 \mu \mathrm{M}$ embelin. The CI values are interpreted as follows: $<1.0$, synergism; 1.0 , additive; and $>1.0$, antagonism. Each experiment was done in triplicate. 
by monitoring polarity changes in the micellar microenvironment [198, 199]. Changes in the vibrational band intensity ratio $\left(\mathrm{I}_{3} / \mathrm{I}_{1}\right)$ with polymer concentration was used to detect the onset of micelle formation, where $I_{3}$ at $338 \mathrm{~nm}$ and $I_{1}$ at $333 \mathrm{~nm}$ correspond to the $(0,2)$ and $(0,0)$ bands, respectively [200]. The CMC obtained at the point of inflection of the $\mathrm{I}_{3} / \mathrm{I}_{1}$ versus the logarithm of polymer concentration curve was approximately $1 \mathrm{mg} / \mathrm{L}$ (data not shown). The particle size distribution of these micelles before and after drug loading was measured by dynamic light scattering with mean particle size in the range of 30 to $50 \mathrm{~nm}$.

Drug encapsulation efficiency and micellar solubility

The film sonication method was chosen for the fabrication of bicalutamide and embelin-loaded micelles since it yields micelles with higher drug loading [201]. Preliminary experiments were performed to optimize formulation parameters such as sonication time, output power and the volume of water used for film hydration. This was done to obtain the highest drug loading possible. A sonication time of $7 \mathrm{~min}$, output power of $25 \mathrm{~W}$ and hydration volume of $5 \mathrm{ml}$ (i.e., a micelle concentration of $4 \mathrm{mg} / \mathrm{ml}$ ) proved optimal.

To determine the maximum amount of drug that could be loaded into the micelles, the theoretical loading of bicalutamide and embelin into PEG-PLA micelles was systematically increased from 1 to $20 \% \mathrm{w} / \mathrm{w}$. There was a linear relationship between the amount of embelin incorporated into the micelles with increasing theoretical loading. In contrast, the loading density for bicalutamide could not increase when the theoretical drug loading reached $10 \%$ or higher. At all theoretical loadings, the loading density of embelin was higher than that of bicalutamide especially at theoretical loading above $10 \%$ (Figure 3-6A). From the experimental results, a theoretical loading of 5\% w/w was chosen for all in vitro and in vivo experiments in this study since it was the optimum formulation in terms of drug loading, encapsulation efficiency, and yield.

The effect of micellar solubilization on the aqueous solubility of bicalutamide and embelin-loaded micelles was determined by shaking an excess amount of free drug or 3 $\mathrm{mg}$ of drug-loaded micelles in $1 \mathrm{ml}$ water, respectively. Free bicalutamide and embelin exhibit very low water solubility of $\sim 1 \mu \mathrm{g} / \mathrm{ml}$, respectively. However, bicalutamide and embelin-loaded micelles resulted in a significant increase in drug solubility with increasing theoretical loading (Figure 3-6B). At $10 \% \mathrm{w} / \mathrm{w}$ a 60 -fold increase in water solubility was observed for a micelle concentration of $3 \mathrm{mg} / \mathrm{ml}$. It is noteworthy that at each theoretical loading, the amount of drug brought into solution is essentially equivalent to the amount of drug successfully incorporated in the polymer.

In vivo efficacy assessment of drug-loaded micelles in xenografts

Following in vitro characterization of antiandrogen loaded polymeric micelles in terms of particle size, micellar solubilization, drug loading and inhibitory effect on 

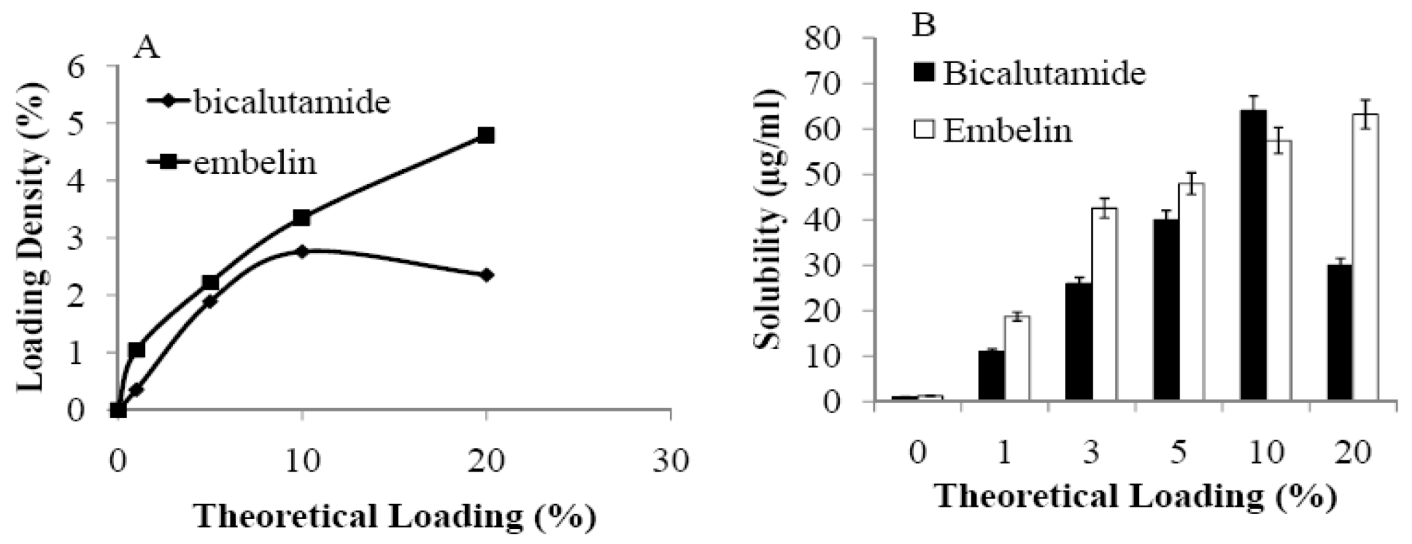

Figure 3-6. Effect of theoretical loading on drug loading density and solubility.

(A) Drug loading- or density and (B) micellar solubilization of bicalutamide and embelin. Water solubility of the drug was determined at $25^{\circ} \mathrm{C}$. Both loading efficiency into PEGPLA micelles and increase in aqueous solubility due to micellar solubization were higher for embelin than bicalutamide. 
LNCaP cell proliferation, we decided to test bicalutamide-loaded micelles in mice bearing LNCaP xenografts. Xenograft flank tumors were induced in 8 week old male $\mathrm{BALB} / \mathrm{C}$ nude mice by subcutaneous injection of 3 million LNCaP cells suspended in 1:1 media and matrigel. When tumors reached approximately $150 \mathrm{~mm}^{3}$, mice were randomized into three groups of 5 mice. Each group was treated with intratumoral injection of saline, bicalutamide suspension and bicalutamide-loaded micelles at the dose of $20 \mathrm{mg} / \mathrm{kg}$ three times a week. Tumors were measured with a caliper prior to each injection, and their volumes calculated using the formula: (width ${ }^{2} \mathrm{x}$ length)/2. As shown in Figure 3-7, tumor growth was effectively regressed upon treatment with bicalutamide up to 20 days post-treatment. Formulation into PEG-PLA polymeric micelles further regressed tumor growth and this effect was more significant in case of bicalulamide.

Unfortunately, these tumors became insensitive to bicalutamide at 20 days after treatment and began to grow. Since antiapoptotic proteins, including XIAP, is known to get upregulated in hormone refractory prostate cancer, we decided to treat these tumors subsequently with emblin, which is an effective XIAP inhibitor. As shown in Figure 3-7, sequential treatment with embelin resulted in regression of hormone insensitive tumors.

\section{Discussion}

Prostate cancer is the second leading cause of death in men in North America. Since androgens play an important role in progression of prostate cancer, androgen ablation and blockade of androgen action are the two most common modalities for treating prostate cancer. Although bicalutamide is one of the most widely used nonsteroidal antiandrogens for treating prostate cancer, it is highly hydrophobic and prolonged exposure leads to drug resistance, the occurrence of hormone refractory prostate cancer and an increased propensity for metastasis [202-205]. Since hormone refractory tumors are resistant to apoptosis and overexpress XIAP, the objective of this study was to see whether small molecule XIAP inhibitor such as embelin can be used to treat bicalutamide irresponsive tumors.

Since the precise cellular mechanism by which bicalutamide induces apoptosis is still not well understood, we first measured the activity of caspases 3, 8 and 9, since caspase 9 upregulation represents mitochondrial pathway and caspase 8 upregulation represents extrinsic pathway [206]. As shown in Figure 3-2, there was dose dependent increase in all three caspases. However, the level was very high in the case of 3 and 9. Our results are in good agreement with the work of Lee et al. [207], who also reported caspase 3 levels to be 5 -fold higher than caspase 8 when $\mathrm{LNCaP}$ cells were treated with bicalutamide. Further, AR gene silencing has been reported to result in massive prostate tumor cell death through mitochondrial pathway regardless of their androgen sensitivity [208]. These results suggest that bicalutamide mainly follows mitochondrial pathway.

Prolonged treatment with bicalutamide is known to convert androgen sensitive prostate cancer cells into apoptosis resistant hormone refractory cells due to overexpression of antiapoptotic genes including Bcl-2, Bcl- $\mathrm{X}_{\mathrm{L}}$ and XIAP [209]. Among 


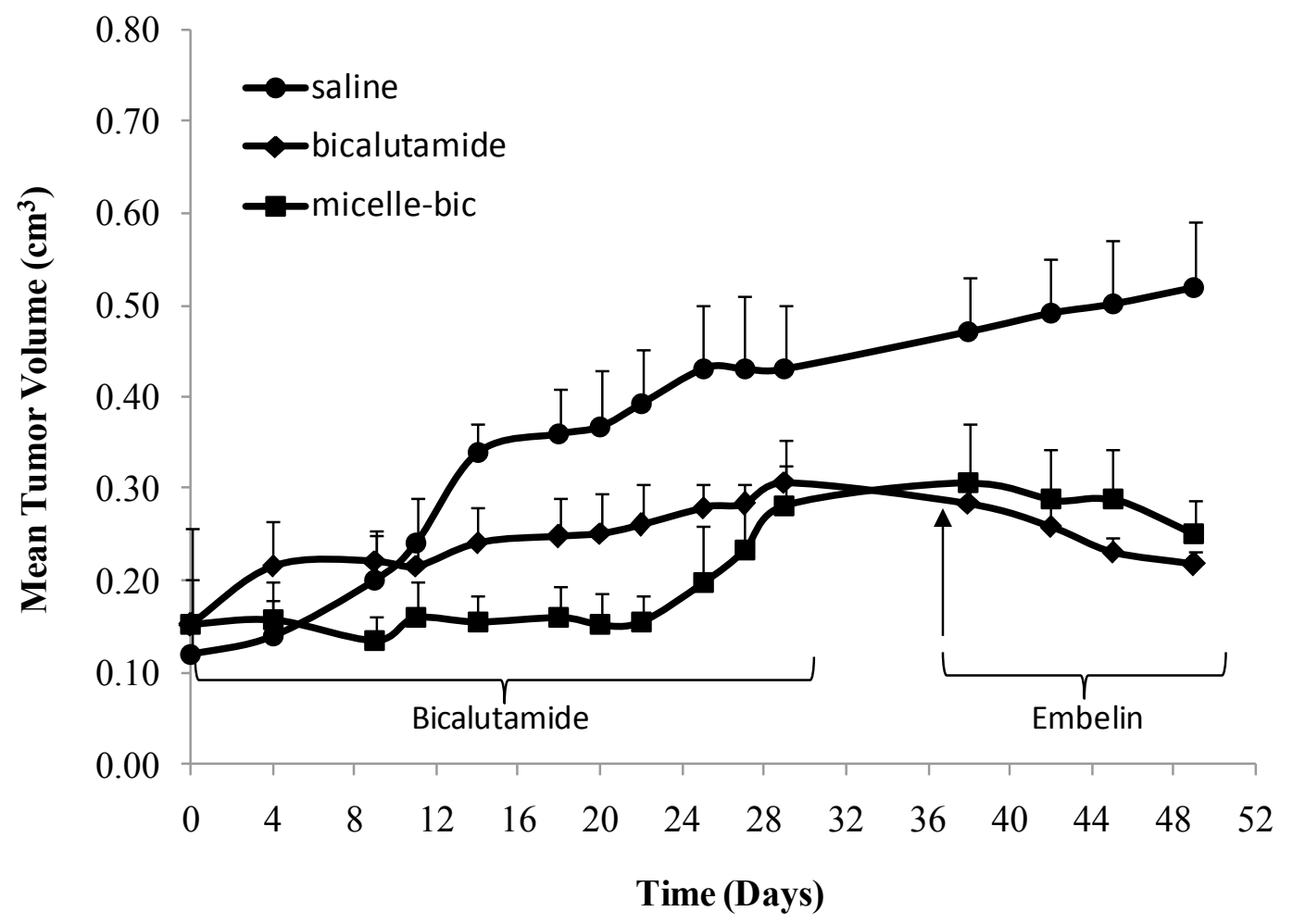

Figure 3-7. Effect of bicalutamide and embelin-loaded micelles on growth of tumors derived from LNCaP prostate cancer cells in nude mice.

Nude mice bearing $150-\mathrm{mm}^{3} \mathrm{LNCaP}$ tumors were given an intratumoral injection of 20 $\mathrm{mg} / \mathrm{kg}$ bicalutamide three times a week and tumor size was measured prior to each injection. Tumor growth regression was significantly higher for bicalutamide-loaded micelles compared to the free drug up to 20 days post-treatment. Since tumors became insensitive to bicalutamide at 20 days after treatment and began to grow, bicalutamide treatment was discontinued and embelin-loaded micelles were administered from day 28, which resulted in regression of hormone insensitive tumors. Points are mean tumor size $(\mathrm{n}=5)$; bars, SE. 
them, XIAP is the most potent in inhibiting apoptosis via inhibition of effector caspases. XIAP inhibitors are known to inhibit caspases, promote mitochondrial permeability, Bcl-2 cleavage and Bak conformational change [210]. Among various XIAP inhibitors, small molecule embelin is quite promising and known to inhibit caspase 9 and effector caspases [191]. Therefore, we determined whether embelin can activate caspases in LNCaP and C4-2 cells (Figure 3-3). We observed increased caspase 3 and 9 activity upon treatment with embelin in both cell lines. Our results are in good agreement with Nikolovska-Coleska et. al. [191] who first showed the ability of embelin to induce caspase 3 and 9 activation in prostate cancer cells by inhibiting the activity of XIAP. While these authors studied the effect of embelin in LNCaP and other prostate cancer cells, they did not examine C4-2 cells and to the best of our knowledge no study using embelin has been done in C4-2 cells. In our study, we found caspase 3 and 9 activation to be lower in C4-2 cells compared to LNCaP cells which we attributed to higher levels of XIAP expression in C4-2 cells. ELISA and real time RT-PCR analysis on LNCaP and C4-2 cells revealed higher baseline XIAP expression in C4-2 cells and treatment with $5 \mu \mathrm{M}$ embelin resulted in a decrease in XIAP expression in both cell lines. It is noteworthy that the decrease in XIAP expression upon treatment with embelin was more pronounced in LNCaP cells compared to C4-2 cells (Figure 3-3). This outcome appears to correlate with the relative caspase 3 and 9 activation levels observed in $\mathrm{LNCaP}$ and C4-2 cells.

We examined the ability of bicalutamide and embelin for treating androgen dependent prostate cancer as well as hormone refractory advanced prostate cancer by observing their effect on cell proliferation. Embelin was more potent than bicalutamide in killing prostate tumor cells irrespective of their androgen status since it was effective in inhibiting the proliferation of androgen dependent $\mathrm{LNCaP}$ and hormone refractory $\mathrm{C} 4-2$ cells (Figure 3-4). Isobologram analysis suggests that the simultaneous administration of bicalutamide and embelin and the sequential treatment of bicalutamide followed by embelin provide synergistic effect on the growth inhibition of hormone refractory C4-2 prostate cancer cells but additive and antagonistic effects in LNCaP cells (Figure 3-5 and Tables 3-1, 3-2 and 3-3). The efficacy of the above treatment schedules possibly stems from bicalutamide sensitizing the cells by androgen ablation to embelin mediated apoptosis. In contrast, the treatment schedule of embelin followed by bicalutamide was additive and antagonistic for both cell lines. However, since the definition of synergism is more stringent in the isobologram method of analysis compared to clinical synergism where drug combinations must simply produce a better tumor response than either drug alone, the observed antagonistic effects in our drug combination study may be equal or superior to that of bicalutamide or embelin alone [211].

Since bicalutamide and embelin are highly hydrophobic, their limited solubility in water can hamper their clinical use by systemic administration. Moreover, the historical use of DMSO to bring water insoluble drugs into true solution for clinical use is of great concern since DMSO is harmful to the liver and kidney and causes dose-dependent hemolysis. A potential approach to addressing these problems is to improve their aqueous solubility using polymeric micelles. Formulation of these drugs into PEG-PLA micelles significantly improved their aqueous solubility and drug loading density increased with 
increasing theoretical loading up to $20 \%$ for embelin and $10 \%$ for bicalutamide (Figure 3-6). Embelin had higher drug loading compared to bicalutamide, possibly due to better hydrogen bonding between the carbonyl group of the PLA hydrophobic coreforming block and the hydroxyl groups of embelin. Similar observations has also been reported for quercetin which has three hydroxyl groups [212]. Differences in their drug loading efficiency and micellar solubilization are also related to their different physicochemical properties as calculated using the Advanced Chemistry Development (ACD/Labs) Software V8.14 for Solaris (Ó 1994-2009 ACD/Labs): The log D (pH =7), $\log \mathrm{P}$ and $\mathrm{pKa}$ values for bicalutamide are 4.94, 9.44 and 11.49, respectively; and those of embelin are 1.20, 5.70 and 2.59, respectively. Micelle size was in the range of 30-50 nm, which is expected to allow enhanced capillary permeability and delivery to tumor and inflammatory sites. Furthermore, the small size of the micelles would facilitate the release of encapsulated drug due to the high surface area to volume ratio.

Following in vitro characterization, bicalutamide-loaded micelles were evaluated in mice bearing LNCaP xenografts. Tumor regression was more significant up to 20 days post-treatment with bicalutamide-loaded micelles compared to free bicalutamide. Unfortunately, tumors became insensitive to bicalutamide afterwards and began to grow. This finding is not surprising, since prostate cancer is known to become resistant to bicalutamide after prolonged treatment, leading to tumor cell proliferation. Subsequent treatment with emblin-loaded micelles resulted in regression of hormone insensitive tumors. This result clearly suggests that combination of bicalutamide with embelin has the potential to be used for treating advanced prostate tumor and metastasis. Sirotnak et al. have also shown synergistic effect of bicalultamide with ZD1839 (Iressa) which inhibits EGFR. However they used extremely high dose $(150 \mathrm{mg} / \mathrm{kg}$ for ZD1839 and 100 $\mathrm{mg} / \mathrm{kg}$ for bicalutamide) and did not measure tumor growth beyond 18 days. In our study there was significant tumor regression up to 20 days when we treated mice with bicalutamide-loaded micelles, but the tumors began to grow beyond 20 days and therefore we stopped treatment with bicalutamide on day 28 and treated mice with embelin a week later [213]. Synergistic effect has also been shown in prostate cancer cell lines between bicalutamide and genistein which is an isoflavone [214].

A major goal of this study was to demonstrate proof of principle that the combination of an androgen receptor antagonist (bicalutamide) and XIAP inhibitor (embelin) was capable of regressing prostate cancer tumors. Since both bicalutamide and embelin are highly hydrophobic and the conventional use of DMSO to solubilize drugs is harmful to the liver and kidney and causes dose-dependent hemolysis, we chose to use polymeric micelles. While the drug solubilization results are not dramatic, they were suitable for intratumoral injections for our xenograft models. For future studies involving systemic administration, we plan to optimize the selection of the hydrophobic block of our copolymer to increase the "cargo" space and enhance drug solubilization. Our laboratory is currently synthesizing polymers to address this issue and preliminary data suggests it may be able to enhance solubilization several fold compared to the poly lactic acid hydrophobic core used in this study. We are also conjugating targeting ligands to the polymeric micelles to enhance site-specific delivery after systemic administration. In 
conclusion, micellar delivery of antiandrogen and XIAP inhibitors has potential to treat advanced prostate cancer. 


\section{CHAPTER 4. SELF-ASSEMBLING METHOXYPOLY(ETHYLENE GLYCOL)- B-POLY(CARBONATE-CO-L-LACTIDE) BLOCK COPOLYMERS FOR DRUG DELIVERY*}

\section{Introduction}

Androgens are known to play a pivotal role in the development and maintenance of the prostate by interacting with the androgen receptor (AR) [215]. Consequently, androgen ablation, especially the use of antiandrogens, has been used as a standard treatment for men with prostate cancer. Among various antiandrogens, bicalutamide $\left(\right.$ Casode $\left.^{\mathrm{TM}}\right)$ is the most widely used non-steroidal antiandrogen for treating early stage prostate cancer due to its relatively long half life and tolerable side effects [216]. However, bicalutamide exhibits poor aqueous solubility $(5 \mu \mathrm{g} / \mathrm{mL})$, which results in poor and variable drug absorption across the gastro intestinal tract. Furthermore, bicalutamide cannot be administered systemically, as traditional approaches to increase its aqueous solubility using solubilizing agents such as dimethyl sulfoxide (DMSO) and Cremophor EL is not practical in humans due to hemolysis, acute hypertensive reactions and neuropathies [217].

One way of improving the solubility of hydrophobic drugs and in particular bicalutamide is by using polymeric micelles. Micelles are attractive drug delivery vehicles primarily because they can solubilize hydrophobic drugs in their core leading to improved bioavailability and drug stability. Furthermore, micelles are capable of preventing drug degradation, minimizing the adverse effects of the drug on visceral organs and have the possibility of being made site-specific [218]. A number of amphiphilic diblock copolymers composed of polyethylene glycol (PEG) and various biodegradable hydrophobic cores capable of forming micelles have been reported in the literature. Examples include: poly (ethylene glycol)-b-poly (aspartic acid) [PEG-b-PAA] [219], poly (ethylene glycol)-b-poly(lactide-co-glycolic acid) [PEG-b-PLGA] [220], poly (ethylene glycol)-b-poly(caprolactone) [PEG-b-PCL] [221] and poly (ethylene glycol)-bpoly(D,L-lactide) [PEG-b-PDLLA] [222]. Key properties of these micelle systems such as size, stability, drug release kinetics and drug loading have also been well studied.

We have recently demonstrated the feasibility of using PEG-b-PDLLA micelles to increase the aqueous solubility of bicalutamide. Although we were able to increase the aqueous solubility of bicalutamide, we observed only moderate drug loading levels which may not be high enough for systemic administration. To solve this problem, the focus of the present study was to specifically design and develop a new family of biodegradable amphiphilic copolymers to improve the aqueous solubility of bicalutamide by enhancing its loading levels. Our strategy involves modifying the polyester component

*Danquah M, Fujiwara T, Mahato RI. Self-assembling methoxypoly(ethylene glycol)-bpoly(carbonate-co-L-lactide) block copolymers for drug delivery. Biomaterials.

2010;31:2358-70. 
of the well established PEG-b-polyester copolymer into polyester/polycarbonate copolymer system. The semicrystalline poly (L-lactide) was chosen as the polyester block because it is FDA approved, possesses good mechanical properties which may provide adequate stability to the micelle system and is known for its application as a drug delivery material [223-225]. For the carbonate block we selected the cyclic 5-methyl-5benzyloxycarbonyl-1,3-dioxane-2-one carbonate monomer. 5-methyl-5benzyloxycarbonyl-1,3-dioxane-2-one is a modification of 5-benzyloxycarbonyl-1,3dioxane-2-one which is an intermediate in the synthesis of numerous antiviral compounds [226]. This carbonate monomer was chosen since polycarbonates are biodegradable, exhibit low toxicity and possess tunable mechanical properties [227-229]. Furthermore, polycarbonate degrades into carbon dioxide and benzyl alcohol, which unlike the degradation products of poly (L-lactide) [e.g. lactic acid] are less acidic, has less effect on microenvironment $\mathrm{pH}$ and as such will not result in local inflammation. We hypothesize that the introduction of the carbonate monomer would provide additional degrees of freedom to tailor a micelle delivery system that is relatively stable, exhibits improved sustained release and has a hydrophobic core that is more compatible with bicalutamide leading to enhanced drug loading.

Improvement in the extent of compatibility between a drug and the core forming block of the micelle may translate into superior encapsulation efficiency [163-166]. A number of groups have explored the possibility of predicting drug solubilization in micelles based on thermodynamics and found their predictions to closely approximate experimental results [164, 167-169]. Polymer/drug compatibility may be characterized by the Flory-Huggins interaction parameter $\left(\chi_{\mathrm{FH}}\right)$ which accounts for the forces of interaction between the polymer and the drug; and low $\chi_{\mathrm{FH}}$ values suggest that the polymer is thermodynamically a good solvent for the drug. To design a micelle system with improved drug loading, we first performed an in silico study using the Molecular Pro Software to assess the Flory-Huggins interaction parameter $\left(\chi_{\mathrm{FH}}\right)$ between bicalutamide and poly(L-lactide) (PLLA) and a series of poly(carbonate-co-lactide) copolymer with varying carbonate to lactide ratios based on group contribution method.

In this study, a series of poly (ethylene glycol)-b-poly(carbonate-co-lactide) copolymers were synthesized and characterized. Also, the influence of carbonate content on key micelle properties such as size, drug loading, stability and release kinetics was investigated. Furthermore, the microstructure of the micelle core block was analyzed to examine the influence of co-monomer sequence distribution on the physicochemical properties of the copolymer and thermal analysis was used to elucidate the impact of carbonate introduction on the crystalline or amorphous nature of the hydrophobic core. Finally, the efficacy of bicalutamide-loaded micelles was assessed in lymph node adenocarcinoma ( $\mathrm{LNCaP}$ ) human prostate cancer cell lines. 


\section{Materials and Methods}

Materials

2,2-bis(hydroxymethyl) propionic acid, methoxy poly(ethylene glycol) (mPEG, $M \mathrm{n}=5000$, PDI $=1.03)$, stannous 2-ethylhexanoate $\left(\mathrm{Sn}(\mathrm{Oct})_{2}\right)$, dicyclohexycarbodiimide (DCC), dimethylaminopyridine (DMAP) and benzyl bromide were purchased from Sigma Aldrich (St. Louis, MO) and used as received. L-lactide (LA) was purchased from PURAC Biochem bv (Gorinchem, The Netherlands) and recrystallized from ethyl acetate several times. All other reagents were obtained from Sigma Aldrich and used without further purification.

Computation of solubility and Flory-Huggins interaction parameters $\left(\chi_{\mathrm{FH}}\right)$ of bicalutamide with core of peg-b-plla/ peg-b-poly(carbonate-co-lactide) micelles

The Flory-Huggins interaction parameter $\left(\chi_{\mathrm{FH}}\right)$ which characterizes polymer-drug compatibility was calculated using Equations 4-1 and 4-2:

$$
\begin{gathered}
\Delta=\left[\left(\delta_{d}-\delta_{p}\right)_{\text {polarity }}^{2}+\left(\delta_{d}-\delta_{p}\right)_{\text {dispersion }}^{2}+\left(\delta_{d}-\delta_{p}\right)_{\text {hydrogen }}^{2}\right]^{\frac{1}{2}} \\
\chi_{F H}=\frac{\Delta^{2} V_{d}}{R T}
\end{gathered}
$$

where $\Delta^{2}$ is the solubility difference between the drug (d) and the core of the polymeric micelle (p). $V_{d}$ is the molar volume of the drug, $T$ is the temperature in Kelvin and $R$ is the gas constant.

The Hansen partial solubility parameters $\left.\left[\left(\delta_{\mathrm{x}}\right)_{\mathrm{d}},\left(\delta_{\mathrm{x}}\right)_{\mathrm{p}},\left(\delta_{\mathrm{x}}\right)_{\mathrm{h}}\right)\right]$ for the drug (bicalutamide) and the hydrophobic block of PEG-b-PLLA and PEG-b-poly(carbonateco-lactide) [PEG-b-P(CB-co-LA)] copolymers used in equation 1 were estimated using the Molecular Modeling Pro software from ChemSW (Fairfield, CA). This software approximates solubility parameters using the Hansen theory of solubility group contribution method. $\left(\delta_{x}\right)_{\mathrm{d}},\left(\delta_{x}\right)_{\mathrm{p}}$ and $\left(\delta_{x}\right)_{\mathrm{h}}$ refers to the partial solubility parameters accounting for Van der Waals forces of dispersion between atoms, permanent dipoledipole forces between molecules and the proclivity of molecules hydrogen bonding, respectively. Here, subscript $x$ refers to the drug or polymer core.

Synthesis of 5-Methyl-5-benzyloxycarbonyl-1,3-dioxane-2-one

A mixture of 2,2-bis(hydroxymethyl)propionic acid (22.5 g, $0.168 \mathrm{~mol})$, potassium hydroxide ( $88 \%$ assay; $10.75 \mathrm{~g}, 0.169 \mathrm{~mol}$ ), and dimethylformamide (DMF) $(125 \mathrm{~mL})$ was heated to $100^{\circ} \mathrm{C}$ for $1 \mathrm{~h}$ with stirring at which point homogenous potassium salt solution was formed. Benzyl bromide (34.5 g, $0.202 \mathrm{~mol})$ was added 
dropwise to the warm solution, and stirring was continued at $100^{\circ} \mathrm{C}$ for $16 \mathrm{~h}$. Upon completion of the reaction, the mixuture was cooled and the solvent was removed under vacuum. The residue was dissolved in ethyl acetate $(150 \mathrm{~mL})$, hexanes $(150 \mathrm{~mL})$, and water $(100 \mathrm{~mL})$. The organic layer was retained, washed with water $(100 \mathrm{~mL})$, dried $\left(\mathrm{Na}_{2} \mathrm{SO}_{4}\right)$, and evaporated. The resulting solid was recrystallized from toluene to give pure benzyl 2,2-bis(methylol)propionate, as white crystals (20 g, 58\%).

Benzyl 2,2-bis(methylol)propionate $(11.2 \mathrm{~g}, 0.05 \mathrm{~mol})$ was dissolved in pyridine ( $25 \mathrm{~mL}, 0.3 \mathrm{~mol}$ ) and $\mathrm{CH}_{2} \mathrm{Cl}_{2}(150 \mathrm{~mL})$, and the solution was chilled to $-78^{\circ} \mathrm{C}$ under $\mathrm{N}_{2}$. A solution of triphosgene $(7.5 \mathrm{~g}, 25.0 \mathrm{mmol})$ in $\mathrm{CH}_{2} \mathrm{Cl}_{2}$ was added dropwise over $1 \mathrm{~h}$, after which the reaction mixture was stirred at room temperature for $2 \mathrm{~h}$. The reaction was quenched by the addition of saturated aqueous $\mathrm{NH}_{4} \mathrm{Cl}(75 \mathrm{~mL})$. Subsequently, the organic layer was washed with $1 \mathrm{M}$ aqueous $\mathrm{HCl}(3 \times 100 \mathrm{~mL})$, saturated aqueous $\mathrm{NaHCO}_{3}(1 \times 100 \mathrm{~mL})$, dried $\left(\mathrm{Na}_{2} \mathrm{SO}_{4}\right)$, filtered and evaporated to give 5-methyl-5benzyloxycarbonyl-1,3-dioxane-2-one as a white solid (10.6 g, 95\%). The ensuing solid was recrystallized from ethyl acetate prior to polymerization.

Synthesis of peg-b-poly(carbonate-co-lactide)

Stannous 2-ethylhexanoate ( $10 \mathrm{~mol} \%$ relative to $\mathrm{mPEG}$ ) was added to the mixture of prescribed quantities of PEG, lactide and base monomer in a dried polymerization flask under the protection of nitrogen atmosphere. The reaction mixture was heated to $130^{\circ} \mathrm{C}$ for $24 \mathrm{~h}$ with stirring. Afterward, the product was cooled to room temperature to terminate the reaction. The copolymer was dissolved in chloroform and precipitated in a large amount of diethyl ether and hexane (1:2), filtered and dried under vacuum at room temperature.

Polymer characterization

\section{Nuclear magnetic resonance (NMR)}

${ }^{1} \mathrm{H}$ NMR spectra were recorded on a JOEL $\left(270 \mathrm{MHz}, \mathrm{T}=25^{\circ} \mathrm{C}\right)$ and ${ }^{13} \mathrm{C}$ spectra were recorded with a Varian $\left(500 \mathrm{MHz}, \mathrm{T}=25^{\circ} \mathrm{C}\right)$ using deuterated chloroform $\left(\mathrm{CDCl}_{3}\right)$ and deuterated dimethyl sulfoxide (DMSO- $\left.\mathrm{d}_{6}\right)$, respectively as solvents. The chemical shifts were calibrated using tetramethylsilane as an internal reference and given in parts per million.

\section{Gel permeation chromatography $(G P C)$}

A Shimadzu 20A GPC system equipped with two Jordi Gel DVB500 columns and a differential refractive index detector was used to determine the molecular weight and polydispersity index (PDI) of the copolymers. Tetrahydrofuran (THF) was used as eluent 
at a flow rate of $1 \mathrm{~mL} / \mathrm{min}$ at $35^{\circ} \mathrm{C}$. A series of narrow polystyrene standards $(900-$ $100,000 \mathrm{~g} / \mathrm{mol}$ ) were used for calibration and the data was processed using a LCSolution ver.1.21 GPC option software.

\section{Differential scanning calorimetry (DSC)}

A TA Instruments DSC Q 2000 module was used to determine the thermal properties of the synthesized copolymers. Samples were placed in aluminum pans under nitrogen heated from $25^{\circ} \mathrm{C}$ to $100^{\circ} \mathrm{C}$, cooled to $-90^{\circ} \mathrm{C}$ to remove thermal history and heated to $100^{\circ} \mathrm{C}$ at a rate of $10^{\circ} \mathrm{C} / \mathrm{min}$.

Critical micelle concentration (CMC)

Fluorescence spectroscopy was used to estimate the critical micelle concentration (CMC) of PEG-b-PLLA and PEG-b-P(CB-co-LA) copolymer using pyrene as a hydrophobic fluorescent probe. Twelve samples of PEG-b-PLLA or PEG-b-P(CB-coLA) dissolved in methanol with concentrations ranging from $1 \times 10^{-8}$ to $1 \mathrm{~g} / \mathrm{L}$ were prepared and allowed to equilibrate with a constant pyrene concentration of $6 \times 10^{-7} \mathrm{M}$ for $48 \mathrm{~h}$ at room temperature. The fluorescence spectra of pyrene were recorded with a Molecular Devices SpectraMax M2/M2e spectrofluorometer (Sunnyvale, CA). An excitation wavelength of $390 \mathrm{~nm}$ was used and the emission spectra recorded from 320 to $450 \mathrm{~nm}$ with both bandwidths set at $2 \mathrm{~nm}$. Peak height intensity ratio $\left(\mathrm{I}_{3} / \mathrm{I}_{1}\right)$ of the third peak $\left(\mathrm{I}_{3}\right.$ at $\left.338 \mathrm{~nm}\right)$ to the first peak $\left(\mathrm{I}_{1}\right.$ at $\left.333 \mathrm{~nm}\right)$ was plotted against the logarithm of polymer concentration. The value of the $\mathrm{CMC}$ was obtained as the point of intersection of two tangents drawn to the curve at high and low concentrations, respectively.

Preparation of bicalutamide-loaded micelles

Bicalutamide-loaded micelles were prepared using the film sonication method as previously described with slight modifications [230]. In brief, $5 \mathrm{mg}$ of bicalutamide and $95 \mathrm{mg}$ of PEG-b-PLLA or PEG-b-P(CB-co-LA) was dissolved in $5 \mathrm{ml}$ chloroform. The mixture was vortexed for 5 min to ensure homogeneity and the solvent evaporated under $\mathrm{N}_{2}$ flow. The resulting film was hydrated in $10 \mathrm{~mL}$ phosphate buffered saline (PBS) to yield a final concentration of $10 \mathrm{mg} / \mathrm{mL}$ and sonicated for $10 \mathrm{~min}$ using a Misonix ultrasonic liquid processor (Farmingdale, NY) with an amplitude of 50. The ensuing formulation was then centrifuged at 5,000 rpm for $10 \mathrm{~min}$ to separate micelles from residual free drug. Subsequently, the supernatant was filtered using a $0.22 \mu$ m nylon filter. The micelle preparation was lyophilized for $48 \mathrm{~h}$ and stored at $4{ }^{\circ} \mathrm{C}$ to prolong shelf-life and avoid untimely release of the drug. 
Drug loading and encapsulation efficiency

Drug loading was determined as follows: $100 \mathrm{mg}$ lyophilized bicalutamide-loaded micelles were dissolved in chloroform and the drug present in solution measured by ultraviolet spectroscopy. The weight of drug loaded in the micelles was calculated using a calibration curve and background absorbance interference from PEG-b-PLLA or PEG-b$\mathrm{P}(\mathrm{CB}-\mathrm{co}-\mathrm{LA})$ copolymer was accounted for by measuring the absorbance of blank PEGb-PLLA or PEG-b-P(CB-co-LA) under the same conditions. Drug loading content and encapsulation efficiency were then determined using Equations 4-3 and 4-4 as follows:

$$
\begin{gathered}
\text { drug loading density }=\frac{\text { weight of drug in micelle }}{\text { weight of micelle }} \times 100 \% \\
\text { drug encapsulation efficiency }=\frac{\text { weight of drug in micelle }}{\text { weight of drug originally fed }} \times 100 \%
\end{gathered}
$$

Particle size distribution

Mean particle size and size distribution of drug-loaded micelles were determined by dynamic light scattering (DLS) using a Zetasizer (Malvern Instruments, Worcestershire, UK) at a $1 \mathrm{mg} / \mathrm{mL}$ polymer concentration. Samples were analyzed at room temperature with a $90^{\circ}$ detection angle and the mean micelle size was obtained as a Z-average. All measurements were repeated seven times and reported as the mean diameter \pm SD for triplicate samples.

\section{Transmission electron microscopy}

Micelles prepared using PEG-b-P(CB-co-LA) copolymer were visualized using a JEM-100S (Japan) transmission electron microscope (TEM). Five microliters of micelle suspension was loaded on a copper grid, followed by blotting of excess liquid and airdried before negative staining with $1 \%$ uranyl acetate. The grid was visualized under the electron microscope at $60 \mathrm{kV}$ and magnifications ranging from 50,000x to 100,000x.

Bicalutamide release from micelles

The dialysis technique was employed to study the release of bicalutamide from the various copolymer micelles in PBS ( $\mathrm{pH}$ 7.2). Bicalutamide-loaded micelles with a final bialutamide concentration of $0.2 \mathrm{mg} / \mathrm{ml}$ were placed into a dialysis membrane with a molecular weight cut-off of $8000 \mathrm{Da}$ and dialyzed against $50 \mathrm{ml} \mathrm{PBS}(\mathrm{pH} 7.2)$ in a thermo-controlled shaker with a stirring speed of $100 \mathrm{rpm} .1 \mathrm{ml}$ samples were withdrawn at specified times for a period of seven days and assayed with a validated UV spectrophotometer by measuring the absorbance of the solution at $270 \mathrm{~nm}$. The samples taken for measurement were replaced with fresh media and the cumulative amount of 
drug released into the media at each time point was evaluated as the percentage of total drug release to the initial amount of the drug. All experiments were performed in triplicate and the data reported as the mean of the three individual experiments.

In vitro cytotoxicity of bicalutamide-loaded micelles

The ability of bicalutamide and bicalutamide-loaded micelles to inhibit cell proliferation was evaluated using $\mathrm{LNCaP}$ human prostate cell line. Cells were cultured in RPMI 1640 media supplemented with $2 \mathrm{mM}$ L-glutamine, $10 \%$ fetal bovine serum (FBS) and $1 \%$ antibiotic-antimycotic at $37^{\circ} \mathrm{C}$ in humidified environment of $5 \% \mathrm{CO}_{2}$ and subcultured every 3 days to ensure exponential growth. The cells were then seeded in 96well plates at a density of $5 \times 10^{3}$ viable cells/well and incubated for $48 \mathrm{~h}$ to permit cell attachment. The cells were exposed to bicalutamide dissolved in methanol or bicalutamide-loaded micelles at concentrations ranging from 1 to $100 \mu \mathrm{M}$ for $48 \mathrm{~h}$. At the end of treatment, $20 \mu \mathrm{l}$ of MTT soultion $(5 \mathrm{mg} / \mathrm{ml})$ was added to each well and incubated

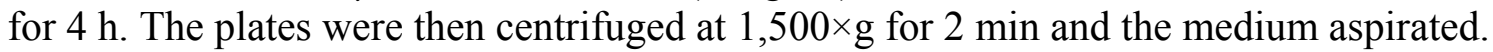
The residual formazan crystals were solubilized with $100 \mu \mathrm{DMSO}$ and the plate analyzed using a microplate reader. The absorbance values were recorded at a test wavelength of $560 \mathrm{~nm}$. Cell viability for a given concentration was expressed as a percentage of the intensity of controls. The data was reported as the mean of triplicate experiments.

\section{Results}

Design of diblock copolymer based on enhanced compatibility between bicalutamide and hydrophobic core

Using PEG-b-PLLA copolymer as a template, a series of diblock copolymers were designed by modifying the PLLA hydrophobic core with a carbonate monomer (i.e., 5-methyl-5-benzyloxycarbonyl-1,3-dioxane-2-one). The goal here was to enhance the compatibility between bicalutamide and the hydrophobic core of the micelle. Since the Flory-Huggins interaction parameter $\left(\chi_{\mathrm{FH}}\right)$ has been shown to be a good indicator of polymer-drug compatibility $[164,166,169]$, we determined $\chi_{\mathrm{FH}}$ for PLLA and P(CB-coLA) using equation 2. The Hansen partial solubility parameters for bicalutamide, PLLA and $\mathrm{P}(\mathrm{CB}-\mathrm{co}-\mathrm{LA})$ were obtained based on the group contribution method using the Molecular Modeling Pro software (Table 4-1). The partial solubility parameters which account for dispersive forces between atoms, permanent dipole interactions between molecules and the tendency of molecules to hydrogen bond were used to calculate the solubility parameter which was subsequently used to compute the interaction parameter. Generally, as $\chi_{\mathrm{FH}}$ approaches zero, compatibility between the polymer and the drug progressively increases since the polymer increasingly becomes a better thermodynamic solvent for the drug, resulting in improved drug solubilzation. The interaction parameter between bicalutamide and PLLA was calculated to be 11.06 while the interaction 
Table 4-1. Calculated Hansen Solubility Parameters of bicalutamide, PLLA and P(CB-co-LA).

\begin{tabular}{llllll}
\hline Drug/polymer & $\begin{array}{l}\boldsymbol{\delta} \text { polarity } \\
\left(\mathbf{J} / \mathbf{c m}^{\mathbf{3}}\right)^{\mathbf{1 / 2}}\end{array}$ & $\begin{array}{l}\boldsymbol{\delta} \text { dispersion } \\
\left(\mathbf{J} / \mathbf{c m}^{\mathbf{3}}\right)^{\mathbf{1 / 2}}\end{array}$ & $\begin{array}{l}\boldsymbol{\delta} \text { hydrogen } \\
\left(\mathbf{J} / \mathbf{c m}^{\mathbf{3}}\right)^{\mathbf{1 / 2}}\end{array}$ & $\begin{array}{l}\text { Total solubility } \\
\text { parameters }^{\mathbf{a}}\end{array}$ & $\chi_{\mathbf{s p}}^{\mathbf{b}}$ \\
\hline Bicalutamide & & & & & - \\
PLLA & 8.89 & 19.66 & 12.43 & 24.90 & 11.06 \\
P(CB-co-LA) & 2.48 & 19.73 & 20.92 & 28.68 & 7.34 \\
\hline
\end{tabular}

${ }^{a}$ Total solubility parameters computed using partial solubility parameters and Equation 4-3. ${ }^{\mathrm{b}}$ Flory-Huggins interaction parameters between bicalutamide and PLLA/ P(CB-co-LA) were calculated using Equation 4-4. 
parameter between bicalutamide and $\mathrm{P}(\mathrm{CB}-\mathrm{co}-\mathrm{LA})$ was computed to be 7.34 . Hence, by introducing a carbonate monomer (5-methyl-5-benzyloxycarbonyl-1,3-dioxane-2-one) into the PLLA hydrophobic core, our design suggested a potential increase in compatibility between bicalutamide and the micelle core and provided a logical justification for the synthesis of the poly (ethylene glycol)-b-poly(carbonate-co-lactide) [PEG-b-P(CB-co-LA)] copolymers.

Synthesis and characterization of 5-Methyl-5-benzyloxycarbonyl-1,3-dioxane-2-one

The cyclic carbonate monomer (5-methyl-5-benzyloxycarbonyl-1,3-dioxane-2one) was synthesized as described by Pratt et. al. with slight modifications (Figure 4-1, Stage 1) [231]. Briefly, 2,2-bis(hydroxymethyl)propionic acid was used to obtain benzyl 2,2-bis(methylol)propionate as an intermediate compound which was subsequently reacted with triphosgene to obtain the cyclic carbonate monomer. The chemical structure of the monomer was confirmed by mass and ${ }^{1} \mathrm{H}$ NMR spectroscopy. From the mass spectrum, the molecular ion peak agreed with the calculated molecular weight of the synthesized monomer (250 g/mol). Also, as depicted in Figure 4-2A, the signal $d$ at 7.5 ppm is characteristic of the phenyl ring protons in the monomer; while the signal $b$ at 4.2 and $4.7 \mathrm{ppm}$ was assigned to the methylene protons present in the carbonate ring. Other peak assignments include signals $a$ and $c$ at 1.3 and $5.2 \mathrm{ppm}$, representing methyl and benzyl $\mathrm{CH}_{2}$ protons, respectively in the monomer.

Synthesis and characterization of peg-b-poly(carbonate-co-lactide) copolymer

The synthesis route of PEG-b-P(CB-co-LA) is delineated in Figure 4-1, Stage 2. A series of PEG-b-P(CB-co-LA) copolymers with varying carbonate $(10,20$ and 40 $\mathrm{mol} \%$ ) content were synthesized by a one pot ring opening polymerization of L-lactide and 5-methyl-5-benzyloxycarbonyl-1,3-dioxane-2-one using methoxy-PEG as the macroinitiator and stannous 2-ethylhexanoate as a catalyst. Among the series, the percent conversion of PEG-b-P(CB-co-LA) copolymer from carbonate was the highest for the 10 mol\% series and was estimated to be $87 \%$ based on ${ }^{1} \mathrm{H}$ NMR spectroscopy. Further ${ }^{1} \mathrm{H}$ NMR analyses was used to verify the composition of PEG-b-P(CB-co-LA) copolymers. From Figure 4-2B, the following resonance peaks were observed for the copolymers at $\delta$ : $1.24\left(\mathrm{CH}_{3}\right.$ in $\mathrm{CB}$ unit, $\left.\mathrm{s}, 3 \mathrm{H}\right) ; \delta: 1.55-1.57\left(\mathrm{CH}_{3}\right.$ in LA unit, $\left.\mathrm{s}, 3 \mathrm{H}\right) ; \delta: 3.65\left(\mathrm{CH}_{2}\right.$ in $\mathrm{PEG}$, $\mathrm{m}, 4 \mathrm{H}) ; \delta: 4.25-4.35\left(\mathrm{CH}_{2}\right.$ in $\mathrm{CB}$ main chain $\left.\mathrm{m}, 4 \mathrm{H}\right) ; \delta: 5.12(\mathrm{CH}$ in LA unit $\mathrm{q}, 1 \mathrm{H}$, and $\mathrm{CH}_{2}$ in $\mathrm{CB}$ side group, $\left.\mathrm{s}, 2 \mathrm{H}\right) \delta: 7.3$ (phenyl, $\mathrm{m}, 5 \mathrm{H}$ ). All signals are assigned as methoxy poly(ethylene glycol) [mPEG], polymerized LA and CB units. The presence of the 7.3 ppm multiplet peak indicates the existence of the carbonate group in the copolymer structure. In addition, the disappearance of the signal $b$ at 4.2 and $4.7 \mathrm{ppm}$ in the carbonate monomer (Figure 4-1A) and the emergence of the multiplet peak at $\delta: 4.25$ $4.35 \mathrm{ppm}$ in the copolymer (Figure 4-1B) confirms the successful ring opening polymerization of the carbonate monomer and the formation of PEG-b-P(CB-co-LA) copolymers. 


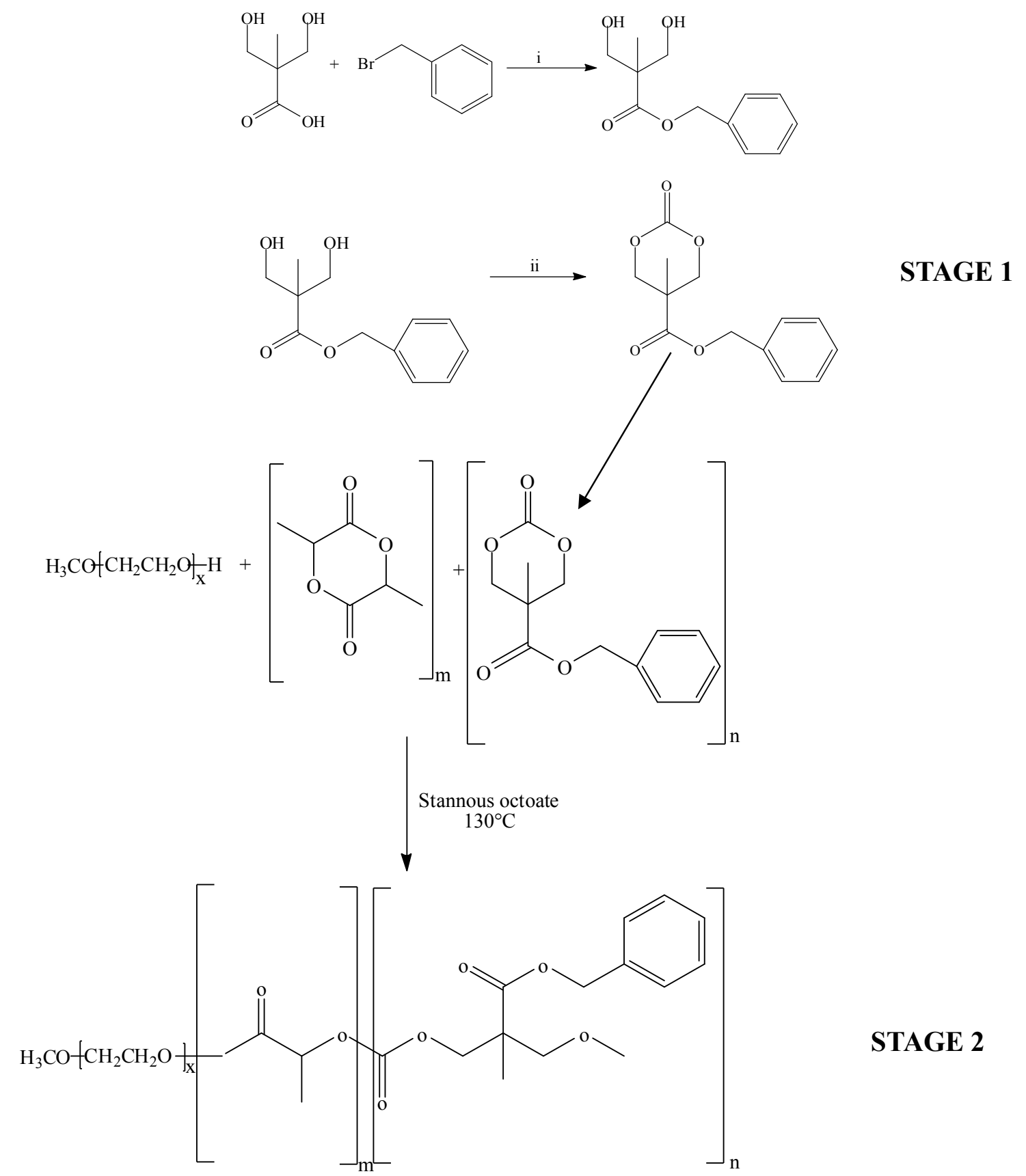

Figure 4-1. Synthesis method of PEG-b-P(CB-co-LA) copolymers.

Stage 1: 5-Methyl-5-benzyloxycarbonyl-1,3-dioxane-2-one (carbonate monomer).

Conditions: (i) $\mathrm{KOH}, \mathrm{DMF}, 100^{\circ} \mathrm{C}, 15 \mathrm{~h}$. (ii) Triphosgene, pyridine, $\mathrm{CH}_{2} \mathrm{Cl}_{2},-78$ to $0^{\circ} \mathrm{C}$. Stage 2: Ring opening polymerization of L-Lactide and 5-Methyl-5-benzyloxycarbonyl1,3-dioxane-2-one initiated by poly (ethylene glycol). 
A
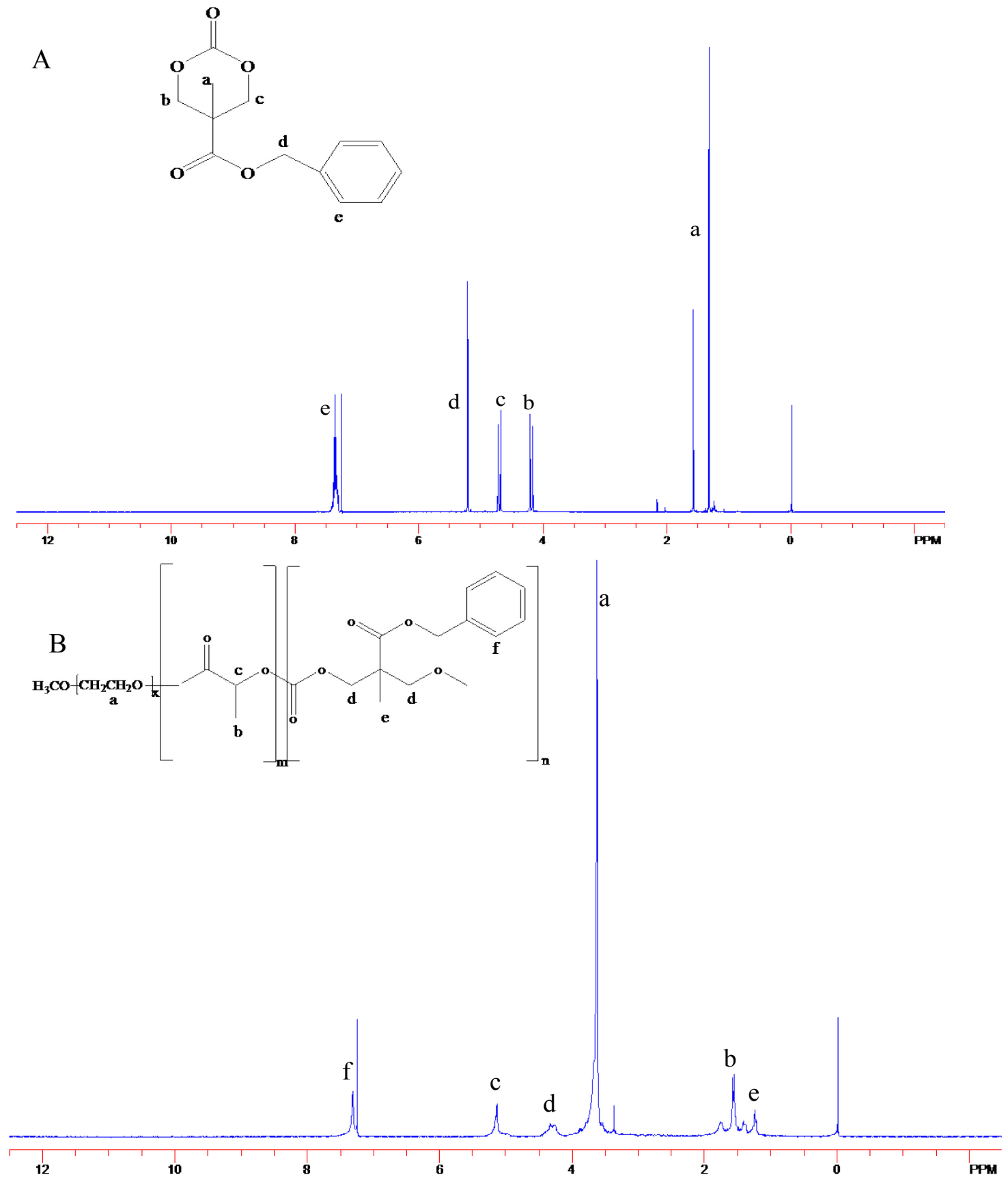

Figure 4-2. $\quad{ }^{1} \mathrm{H}$ NMR spectra in $\mathrm{CDCl}_{3}$ of (A) 5-Methyl-5-benzyloxycarbonyl-1,3dioxane-2-one (carbonate monomer) and (B) $\mathbf{P E G}_{114}-\mathrm{b}-\mathrm{P}\left(\mathrm{CB}_{9}\right.$-co-L $\left.\mathrm{A}_{33}\right)$ copolymer. 
Table 4-2 summarizes some characteristics of the synthesized PEG-b-PLLA and PEG-b-P(CB-co-LA) copolymers. The block copolymer molecular weight was calculated based on a comparative analysis of the four methylene protons of PEG $(\delta=3.65 \mathrm{ppm})$, one methylene proton of lactide $(\delta=5.12 \mathrm{ppm})$ and the five protons associated with the phenyl ring in the carbonate monomer observed in the ${ }^{1} \mathrm{H}$ NMR spectrum. It is worth mentioning that since the lactide proton peak at $5.12 \mathrm{ppm}$ is confounded with the signal from the two protons of the carbonate monomer, two- fifth of the carbonate signal at 7.3 ppm was subtracted from the $5.12 \mathrm{ppm}$ signal to obtain the actual lactide peak intensity used for molecular weight calculations. For the three copolymers studied: $\mathrm{PEG}_{114}-\mathrm{b}-$ $\mathrm{P}\left(\mathrm{CB}_{5}\right.$-co-LA $\left.\mathrm{L}_{32}\right), \mathrm{PEG}_{114}-\mathrm{b}-\mathrm{P}\left(\mathrm{CB}_{8}\right.$-co-LA $\left.\mathrm{LA}_{24}\right)$ and $\mathrm{PEG}_{114}-\mathrm{b}-\mathrm{P}\left(\mathrm{CB}_{9}\right.$-co-LA $)$; the molecular weight were $8604 \mathrm{~g} \mathrm{~mol}^{-1}, 8679 \mathrm{~g} \mathrm{~mol}^{-1}$ and $7510 \mathrm{~g} \mathrm{~mol}^{-1}$, respectively. Hence, the calculated molecular weight from ${ }^{1} \mathrm{H}$ NMR spectroscopy was less than the predicted value of $10,000 \mathrm{~g} / \mathrm{mol}$. The computed molecular weights suggests the degree of polymerization (DP) of the $\mathrm{P}(\mathrm{CB}-\mathrm{Co}-\mathrm{LA})$ core to be lower than the theoretical value. The calculated DP for 10, 20 and $40 \mathrm{~mol} \%$ carbonate is 37,32 and 14, respectively; while the theoretical DP for 10, 20 and $40 \mathrm{~mol} \%$ carbonate is 54, 44 and 34, respectively. Gel permeation chromatography (GPC) analysis revealed PEG-b-P(CB-co-LA) copolymers to be broader than PEG-b-PLLA as evinced by the polydispersity index $\left(\mathrm{M}_{\mathrm{w}} / \mathrm{M}_{\mathrm{n}}=1.37\right.$, 1.40 and 1.51) for PEG-b-P(CB-co-LA) copolymers compared to $\mathrm{M}_{\mathrm{w}} / \mathrm{Mn}=1.07$ for PEG-b-PLLA with the breadth of polydispersity increasing with carbonate. Furthermore, PEG-b-P(CB-co-LA) copolymers exhibited a unimodal peak and a representative plot comparing the GPC chromatograms for PEG-b-PLLA and $\mathrm{PEG}_{114}-\mathrm{b}-\mathrm{P}\left(\mathrm{CB}_{8}\right.$-co- $\left.\mathrm{LA}_{24}\right)$ is shown in Figure 4-3. The unimodal peaks observed for the copolymer series suggests that successful copolymerization took place and that the insertion of carbonate and lactide monomers in the $\mathrm{P}(\mathrm{CB}-\mathrm{co}-\mathrm{LA})$ core was random.

Preparation and characterization of peg-b-poly(carbonate-co-lactide) copolymer micelles

The film sonication method described in a previous publication [230] was used to prepare both unloaded and bicalutamide-loaded polymeric micelles from the synthesized PEG-b-PLLA and PEG-b-P(CB-co-LA) copolymers[230]. The size distribution for both unloaded and bicalutamide-loaded polymeric micellar formulations as determined by dynamic light scattering (DLS) was relatively broad with a PDI of approximately 0.2 , reflecting the possible presence of a population of aggregates. Figure 4-4A shows a typical size distribution plot obtained from DLS of PEG-b-P(CB-co-LA) micelles using a polymer concentration of $10 \mathrm{mg} / \mathrm{mL}$. The size distribution is bimodal with a smaller size population $\sim 53 \mathrm{~nm}(58 \%)$ and a larger population $\sim 162 \mathrm{~nm}(42 \%)$. Effective hydrodynamic diameter for both unloaded and bicalutamide-loaded PEG-b-PLLA and PEG-b-P(CB-co-LA) micelles reported as the z-average based on mean intensitiy was determined by DLS. In the case of the unloaded micelles, the average diameter was found to range from 107 to $135 \mathrm{~nm}$, while the average diameter of bicalutamide-loaded micelles ranged from 99 to $123 \mathrm{~nm}$ (Table 4-3). In both cases the $20 \mathrm{~mol} \%$ carbonate copolymer had the largest average diameter while the $40 \mathrm{~mol} \%$ carbonate copolymer had the smallest average diameter. 
Table 4-2. Effect of carbonate content on the molecular weight as determined by ${ }^{1} \mathrm{H}$ NMR and GPC and CMC of micelles at $25^{\circ} \mathrm{C}$.

\begin{tabular}{|c|c|c|c|c|c|c|c|c|}
\hline Block copolymer $^{a}$ & $\begin{array}{l}\text { Carbonate } \\
(\mathrm{mol} \%)^{b}\end{array}$ & $\begin{array}{l}\text { Theoretical } \\
\text { mol. wt. } \\
(\mathrm{g} / \mathrm{mol})\end{array}$ & $\begin{array}{l}\mathbf{M}_{n} \\
(\mathbf{N M R})\end{array}$ & $\begin{array}{l}M_{n} \\
(G P C)\end{array}$ & $\begin{array}{l}M_{w} \\
(G P C)\end{array}$ & $\begin{array}{l}M_{w} / M_{n} \\
(G P C)\end{array}$ & $\begin{array}{l}\text { CMC } \\
(\mathrm{g} / \mathrm{L})\end{array}$ & $\begin{array}{l}\mathrm{CMCx10} \\
\left(\mathrm{mol}^{-1}\right)^{\mathrm{c}}\end{array}$ \\
\hline $\mathrm{PEG}_{114}-\mathrm{b}-\mathrm{PLLA}_{62}$ & 0 & 10000 & 9460 & 9104 & 9741 & 1.07 & 0.03 & 32.95 \\
\hline $\mathrm{PEG}_{114}-\mathrm{b}-\mathrm{P}\left(\mathrm{CB}_{5}-\mathrm{co}-\mathrm{LA}_{32}\right)$ & 10 & 10000 & 8604 & 6228 & 8533 & 1.37 & 0.005 & 8.03 \\
\hline $\mathrm{PEG}_{114}-\mathrm{b}-\mathrm{P}\left(\mathrm{CB}_{8}-\mathrm{co}-\mathrm{LA}_{24}\right)$ & 20 & 10000 & 8679 & 6384 & 8925 & 1.40 & 0.002 & 3.13 \\
\hline $\mathrm{PEG}_{114}-\mathrm{b}-\mathrm{P}\left(\mathrm{CB}_{9}-\mathrm{co}-\mathrm{LA}_{5}\right)$ & 40 & 10000 & 7510 & 5694 & 8573 & 1.51 & 0.004 & 7.02 \\
\hline
\end{tabular}

${ }^{a}$ Subscripts reflect degree of polymerization of each monomer obtained from ${ }^{1} \mathrm{H}$ NMR spectroscopy. ${ }^{\mathrm{b}}$ Mol\% indicates carbonate content in $\mathrm{P}(\mathrm{CB}-\mathrm{co}-\mathrm{LA})$ block copolymer. Molecular weight calculated from ${ }^{1} \mathrm{H}$ NMR spectroscopy,

$\mathrm{M}_{\mathrm{n}, \mathrm{NMR}}=\mathrm{M}_{\mathrm{n}, \mathrm{PEG}}+\mathrm{M}_{\mathrm{n}, \text { Carbonate }}+\mathrm{M}_{\mathrm{n}, \mathrm{L} \text {-Lactide. }}{ }^{\mathrm{c}} \mathrm{CMC}(\mathrm{g} / \mathrm{L})$ normalized with $\mathrm{M}_{\mathrm{n}}$ from GPC. 


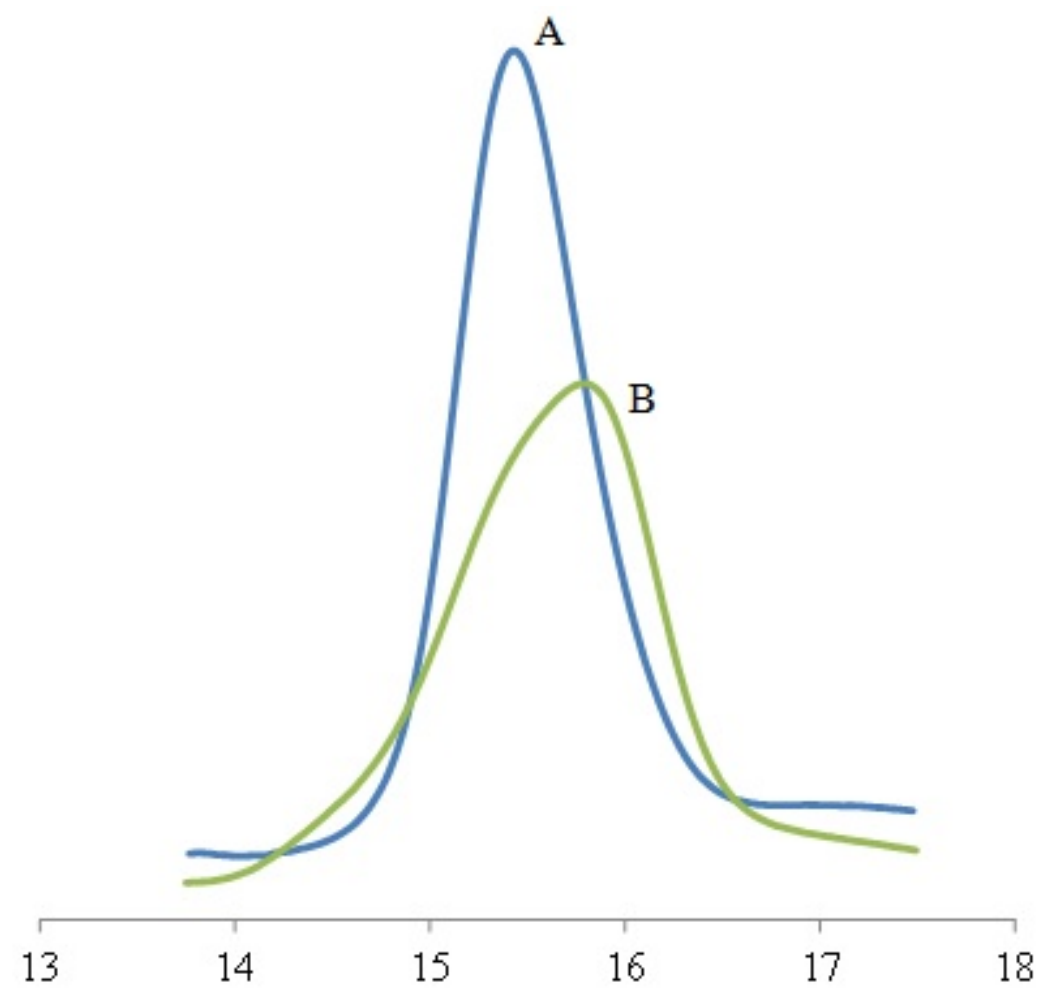

Retention Time (min)

Figure 4-3. Gel permeation chromatograms of PEG-b-PLLA and $P_{E G} 14-b-$ $\mathbf{P}\left(\mathrm{CB}_{9}\right.$-co-LA $\left.\mathbf{A}_{33}\right)$ copolymers.

(A) PEG-b-PLLA [ $\left.\mathrm{M}_{\mathrm{n}}=9460 \mathrm{~g} / \mathrm{mol}, \mathrm{M}_{\mathrm{w}} / \mathrm{M}_{\mathrm{n}}=1.07\right]$ and (B) $\mathrm{PEG}_{114}-\mathrm{b}-\mathrm{P}\left(\mathrm{CB}_{9}-\mathrm{co}_{-}-\mathrm{LA}_{33}\right)$ $\left[\mathrm{M}_{\mathrm{n}}=9720 \mathrm{~g} / \mathrm{mol}, \mathrm{M}_{\mathrm{w}} / \mathrm{M}_{\mathrm{n}}=1.40\right]$ copolymers, both acquired in THF. 

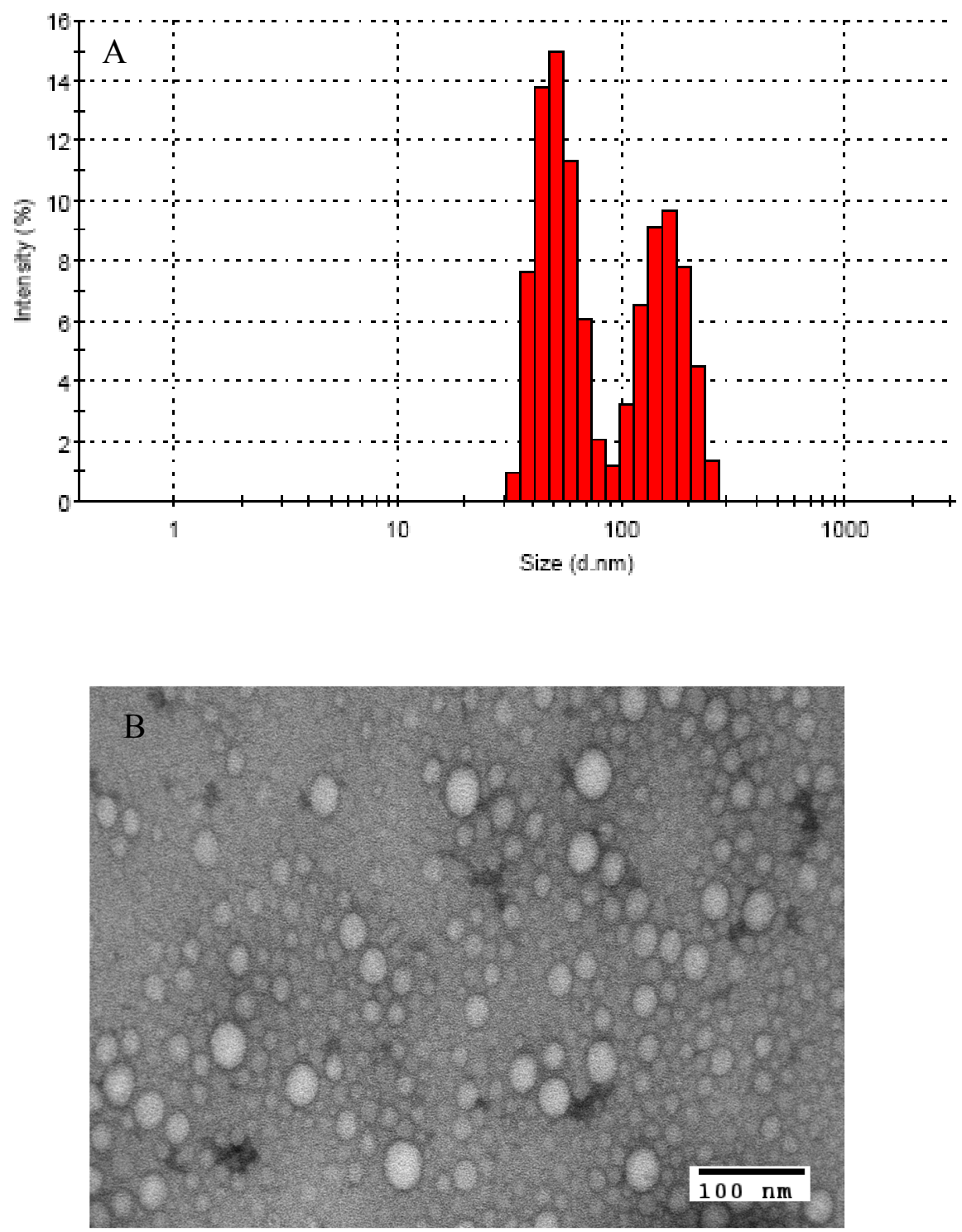

Figure 4-4. Micelle size distribution and surface morphology.

(A) Dynamic light scattering histogram; and (B) transmission electron micrograph of micelle formulated with PEG-b-P(CB-co-LA) copolymer obtained using uranyl acetate staining. 
Table 4-3. Effect of polymer composition on drug loading and particle size of bicalutamide-loaded micelles.

\begin{tabular}{|c|c|c|c|c|}
\hline Block copolymer $^{\text {a }}$ & $\begin{array}{l}\text { Drug loading } \\
(\%) \pm \text { SD }^{b}\end{array}$ & $\begin{array}{l}\text { Encap. efficiency } \\
(\%) \pm \text { SD }\end{array}$ & $\begin{array}{l}\text { Bicalutamide/polymer } \\
\text { core }(\mathrm{mol} \%)\end{array}$ & $\begin{array}{l}\text { Mean diameter } \\
\pm \text { SD (nm) }\end{array}$ \\
\hline $\mathrm{PEG}_{114}-\mathrm{b}-\mathrm{PLLA}_{62}$ & $1.0 \pm 0.31$ & $22.04 \pm 3.82$ & 22 & $110 \pm 0.9$ \\
\hline $\mathrm{PEG}_{114}-\mathrm{b}-\mathrm{P}\left(\mathrm{CB}_{5}-\mathrm{co}-\mathrm{LA}_{32}\right)$ & $3.36 \pm 0.18$ & $72.68 \pm 4.27$ & 876 & $101 \pm 1.0$ \\
\hline $\mathrm{PEG}_{114}-\mathrm{b}-\mathrm{P}\left(\mathrm{CB}_{8}-\mathrm{co}-\mathrm{LA}_{24}\right)$ & $4.10 \pm 0.23$ & $81.96 \pm 2.54$ & 1097 & $123 \pm 1.5$ \\
\hline $\mathrm{PEG}_{114}-\mathrm{b}-\mathrm{P}\left(\mathrm{CB}_{9}-\mathrm{co}-\mathrm{LA}_{5}\right)$ & $1.38 \pm 0.46$ & $27.56 \pm 2.91$ & 779 & $99 \pm 1.4$ \\
\hline
\end{tabular}

${ }^{a}$ Subscripts reflect degree of polymerization of each monomer obtained from ${ }^{1} \mathrm{H}$ NMR spectroscopy.

${ }^{b}$ Percentage of drug loaded into micelles based on 5\% theoretical loading. ${ }^{\mathrm{c}}$ Mean particle size was determined by dynamic light scattering. 
Particle size and surface morphology of these micelles were also determined by transmission electron microscopy (TEM). The polymer micelles have a broad size distribution with mean number average diameter below $50 \mathrm{~nm}$ (Figure 4-4B), which is lower than the z-average diameters obtained from DLS. TEM images also reveal a tendency for micellar aggregation and confirm that PEG-b-P(CB-co-LA) formed true spherical micelles in water with distinct boundaries as anticipated.

The polymer micelles were further characterized by fluorescence spectroscopy to determine the critical micelle concentration (CMC). The CMC of the synthesized block copolymers decreased upon introduction of the carbonate monomer from $\sim 3.3$ to $\sim 0.3$ $\mu \mathrm{M}$ for 0 and $20 \mathrm{~mol} \%$ carbonate, respectively (Figure 4-5 and Table 4-2). The lower $\mathrm{CMC}$ values suggest that the inclusion of the carbonate moiety results in block copolymers thermodynamically more favored to self-assemble. The amount of bicalutamide loaded into micelles was calculated using equation 3 based on a $5 \%$ theoretical loading (i.e., $5 \mathrm{mg}$ drug/ $100 \mathrm{mg}$ polymer). PEG-b-P(CB-co-LA) copolymers exhibited higher drug loading compared to PEG-b-PLLA copolymer (Table 4-3). Among PEG-b-P(CB-co-LA) copolymer series, the highest loading $(4.10 \%)$ was observed for $\mathrm{PEG}_{14}-\mathrm{b}-\mathrm{P}\left(\mathrm{CB}_{8}-\mathrm{co}-\mathrm{LA}_{24}\right)$ copolymers which was at least four-fold better than PEG-bPLLA copolymer with regards to drug loading and had an encapsulation efficiency of approximately $82 \%$. For $\mathrm{PEG}_{114}-\mathrm{b}-\mathrm{P}\left(\mathrm{CB}_{5}-\mathrm{co}-\mathrm{LA}_{32}\right)$ the drug loading was $3.36 \%$ which is about three times higher than that of PEG-b-PLLA and its encapsulation efficiency was roughly $73 \%$. $\mathrm{PEG}_{114}-\mathrm{b}-\mathrm{P}\left(\mathrm{CB}_{9}\right.$-co-LA $)$ copolymer had a drug loading of $1.38 \%$ and an encapsulation efficiency of $28 \%$ which is only slightly better than PEG-b-PLLA which had a drug loading of $1 \%$ and an encapsulation efficiency of $22 \%$. Since the degree of polymerization of $\mathrm{P}(\mathrm{CB}-\mathrm{co}-\mathrm{LA})$ hydrophobic core varied across the series, the drug loading was also assessed on a molar basis with respect to just the hydrophobic core (i.e., mol bicalutamide/mol hydrophobic core) to normalize the data. Here again, $\mathrm{PEG}_{114}$-b$\mathrm{P}\left(\mathrm{CB}_{8}\right.$-co- $\left.\mathrm{LA}_{24}\right)$ had the highest loading $(1097 \%)$, followed by $\mathrm{PEG}_{114}-\mathrm{b}-\mathrm{P}\left(\mathrm{CB}_{5}-\mathrm{co}-\mathrm{LA}_{32}\right)$ and then $\mathrm{PEG}_{114}-\mathrm{b}-\mathrm{P}\left(\mathrm{CB}_{9}-\mathrm{co}-\mathrm{LA}_{5}\right)$ which had $876 \%$ and $779 \%$ loading, respectively. In all cases, the drug loading on a molar basis in PEG-b-P(CB-co-LA) copolymer series was several fold higher than that of PEG-b-PLLA copolymer.

To establish that PEG-b-P(CB-co-LA) micelles are capable of forming core-shell structures, the ${ }^{1} \mathrm{H}$ NMR spectra of micelle samples in deuterated water $\left(\mathrm{D}_{2} \mathrm{O}\right)$ and DMSO-d6 was compared. Typical spectra for $\mathrm{PEG}_{114}-\mathrm{b}-\mathrm{P}\left(\mathrm{CB}_{8}-\mathrm{co}-\mathrm{LA}_{24}\right)$ micelles are shown in Figure 4-6. In $\mathrm{D}_{2} \mathrm{O}$, only the PEG proton peaks can be detected while the signals for PLLA and polycarbonate are not evident. On the contrary, distinct peaks for PLLA and polycarbonate were observed in DMSO-d6 in addition to the PEG signal, suggesting that PEG-b-P(CB-co-LA) micelle form core-shell structures capable of encapsulating bicalutamide. This result is consistent with what is reported in the literature since the PEG forms the corona of the micelle and enhances its solvation in $\mathrm{D}_{2} \mathrm{O}$ while PLLA, polycarbonate and the encapsulated bicalutamide are in the core of the micelle and have restricted mobility in $\mathrm{D}_{2} \mathrm{O}$. 


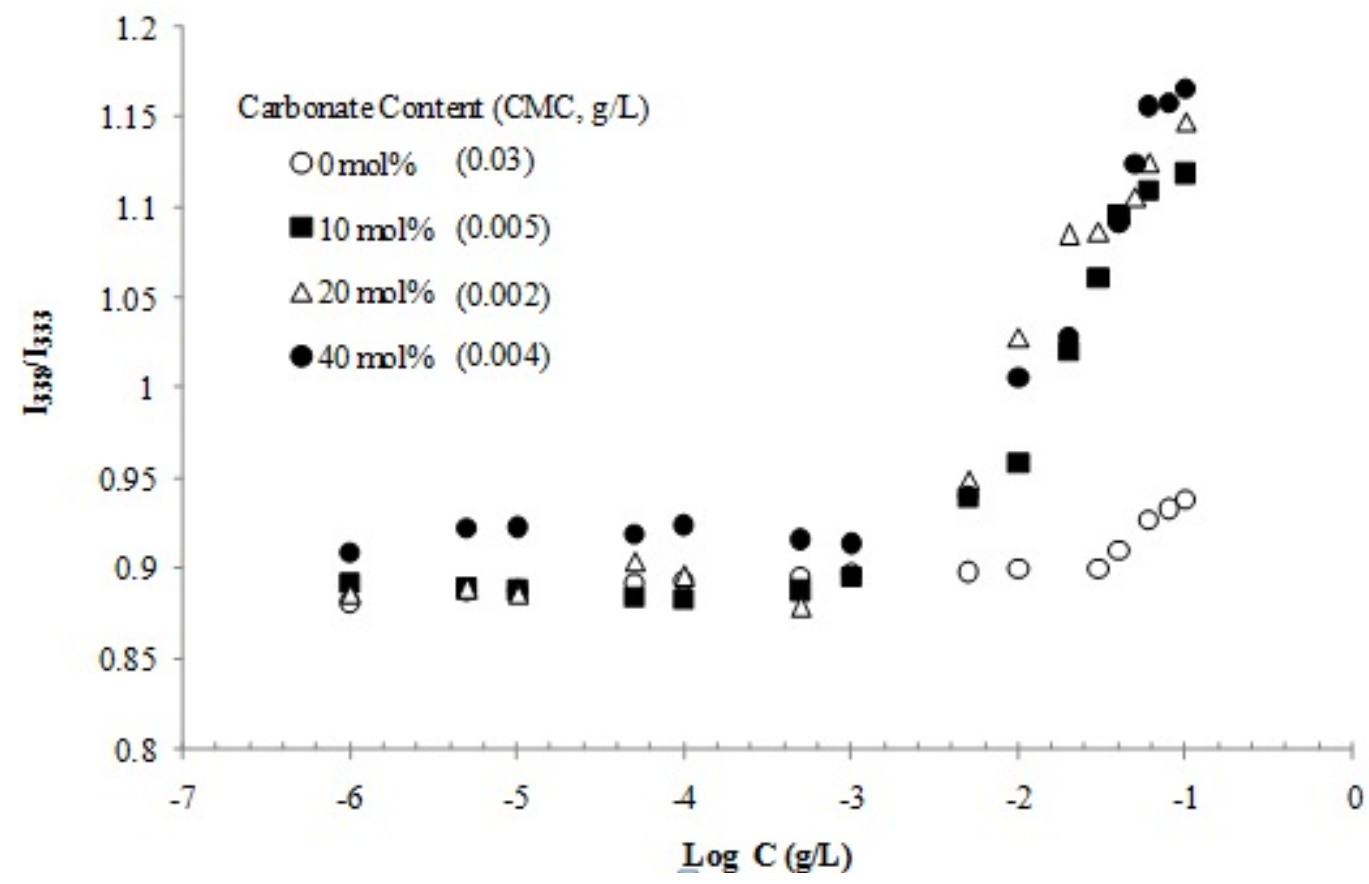

Figure 4-5. Plots of intensity ratio $I_{338} / I_{333}$ from pyrene fluorescence emission spectra versus log concentration (g/L) of PEG-b-PLLA and PEG-b-P(CB-co-LA) copolymers. 

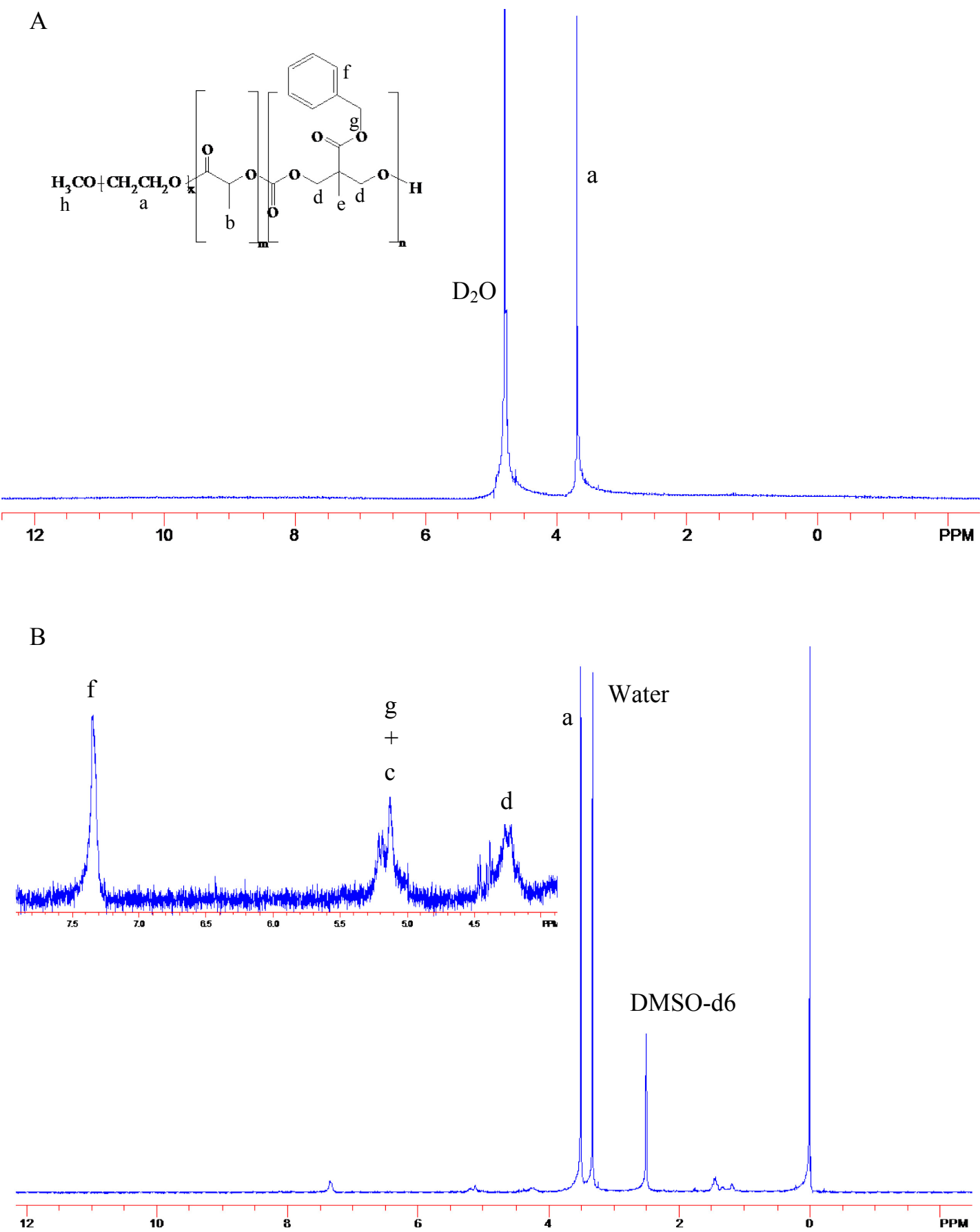

Figure 4-6. $\quad{ }^{1} \mathrm{H}$ NMR spectra of bicalutamide-loaded PEG $_{114-b}-\mathrm{P}\left(\mathrm{CB}_{8}-\mathrm{co}-\mathrm{LA} \mathrm{A}_{24}\right)$ micelles.

(A) In deuterated water $\left(\mathrm{D}_{2} \mathrm{O}\right)$ and $(\mathrm{B})$ in DMSO-d6. 
Sequence analysis

Sequence distribution in a copolymer is frequently analyzed in terms of monomer triads. Considering that the hydrophobic core of the synthesized PEG-b-P(CB-co-LA) copolymers is made up of two monomers: carbonate $(\mathrm{C})$ and lactide $(\mathrm{L})$, this system has eight possible theoretical triads. Prior to the analysis of triads in the copolymer, the complete assignment for the carbonyl peaks in PEG-b-P(CB-co-LA) copolymer based on ${ }^{13} \mathrm{C}$ NMR spectra of homopolymers (PLLA and polycarbonate) was first determined and the results are shown in Figure 4-7A. To elucidate the arrangement of triads in PEG-b$\mathrm{P}(\mathrm{CB}-\mathrm{co}-\mathrm{LA})$ copolymer, ${ }^{13} \mathrm{C}$ NMR spectrum was expanded for clarity (Figure 4-7B). The resonance peak at $169.6 \mathrm{ppm}$ is assigned to the central carbonyl of LLL and LLC triads. Further upfield the resonance peak occurring at $169.45 \mathrm{ppm}$, which is due to the central carbonyl in the CLC and CLL triads. The resonance peak at $154.35 \mathrm{ppm}$ is assigned to the central carbonyl of CCC and CCL triads, while the resonance peak at $154.15 \mathrm{ppm}$ is due to the central carbonyl of the LCL and LCC triads. The above data together with the observed splitting of the carbonyl peaks strongly suggests that the hydrophobic core PEG-b-P(CB-co-LA) is a random copolymer. Having confirmed the formation of random copolymers, the influence of carbonate monomer on the distribution of L-L and C-C sequence was assessed by comparing the ${ }^{13} \mathrm{C}$ NMR spectra of 10, 20 and $40 \mathrm{~mol} \%$ carbonate copolymers. From Figure 4-8, increasing CB contents from 10 to 40 $\mathrm{mol} \%$ decreases L-L sequence and increases $\mathrm{C}-\mathrm{C}$ sequence (particularly for $40 \%$ ).

\section{Thermal analysis}

The thermal properties of the synthesized block copolymers were examined using differential scanning calorimetry (DSC) and the thermograms of PEG-b-PLLA and PEGb-P(CB-co-LA) copolymers are shown in Figure 4-9. Within the temperature range examined $\left(-90\right.$ to $\left.175^{\circ} \mathrm{C}\right)$, two endotherm peaks at 51.5 and $155^{\circ} \mathrm{C}$ were observed for PEG-b-PLLA copolymer. An exotherm peak was also observed occurring at $88.5^{\circ} \mathrm{C}$. To the contrary, only one endotherm peak, the fusion of PEG block, was observed for all the PEG-b-P(CB-co-LA) copolymers which appeared to shift slightly to higher temperatures with increasing carbonate content: $52.1,52.9$ and $53.8^{\circ} \mathrm{C}$ for 10,20 and $40 \mathrm{~mol} \%$, respectively.

In vitro release studies of bicalutamide from micelles

The in vitro bicalutamide release study was performed in phosphate buffered saline (PBS, pH 7.4) at $37^{\circ} \mathrm{C}$ and $100 \mathrm{rpm}$. Figure 4-10 shows the cumulative percentage of bicalutamide released from PEG-b-PLLA ( 0 mol\% carbonate) and PEG-b-P(CB-coLA) [10, 20 and $40 \mathrm{~mol} \%$ carbonate] micelles. From the data, the release of bicalutamide is rapid from the micelles with $0 \mathrm{~mol} \%$ carbonate content compared to micelles formulated using copolymers containing carbonate monomer. About $60 \%$ of the total drug was released within $6 \mathrm{~h}$ for $0 \mathrm{~mol} \%$ carbonate content while the 10, 20 and $40 \mathrm{~mol} \%$ carbonate content had a burst release of $40 \%$. The $0 \mathrm{~mol} \%$ carbonate copolymer used in 
A
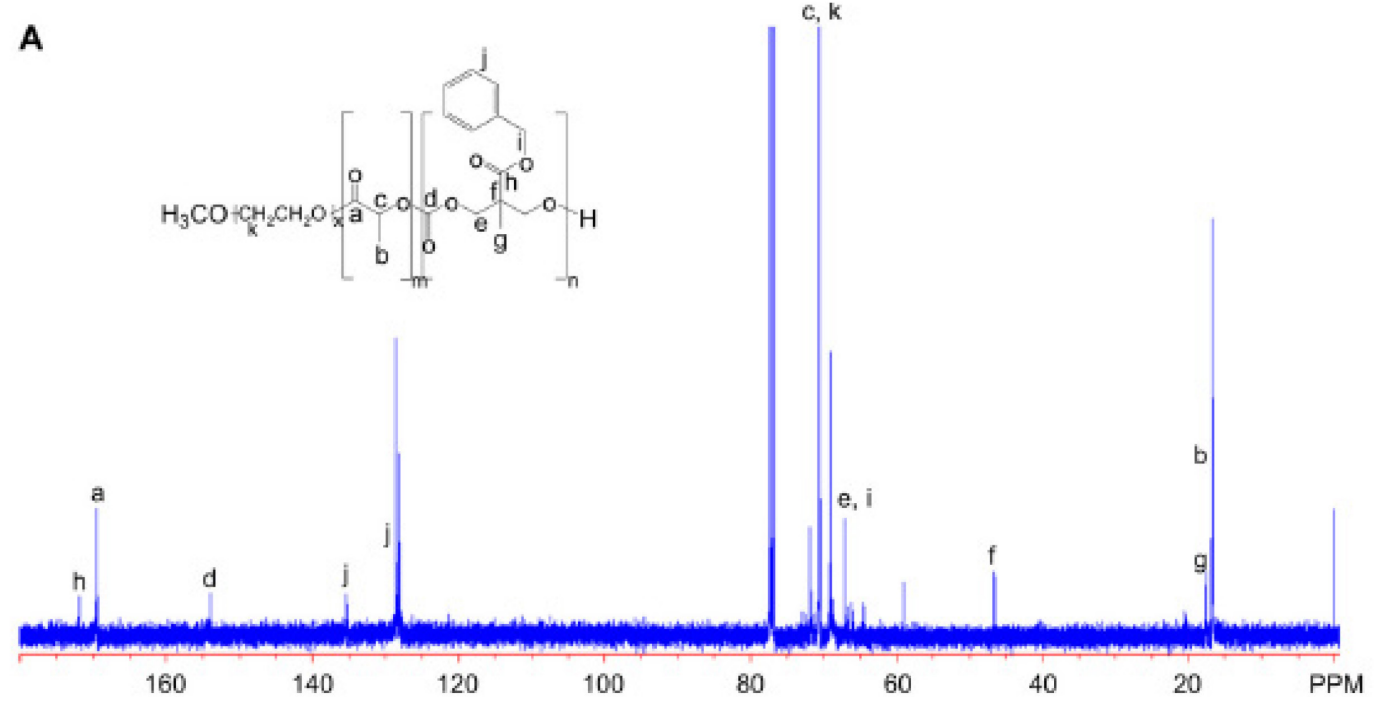

B

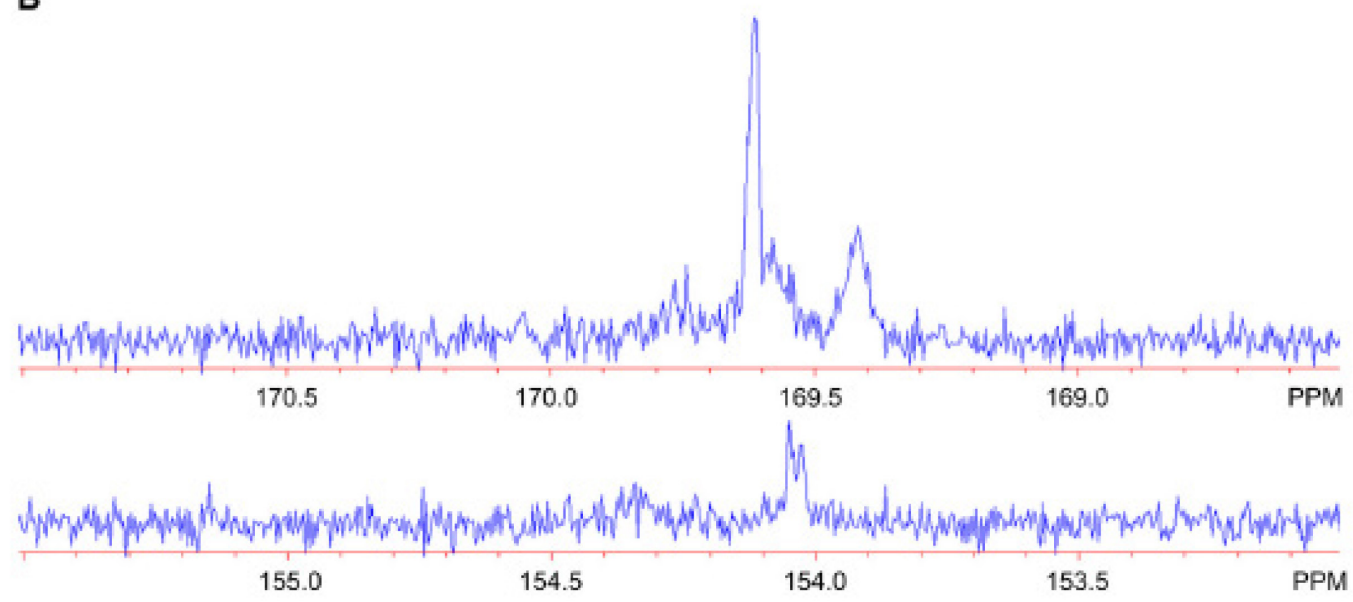

Figure 4-7. $\quad{ }^{13} \mathrm{C}$ NMR spectra of $\mathrm{PEG}_{114}-\mathrm{b}-\mathrm{P}\left(\mathrm{CB}_{8}-\mathrm{Co}-\mathrm{LA}_{24}\right)$ copolymer.

(A) Complete spectrum with peak assignments. (B) Expanded spectrum of specified regions (152 to $170 \mathrm{ppm}$ ) illustrating carbonyl regions of interest for sequence distribution analysis. 


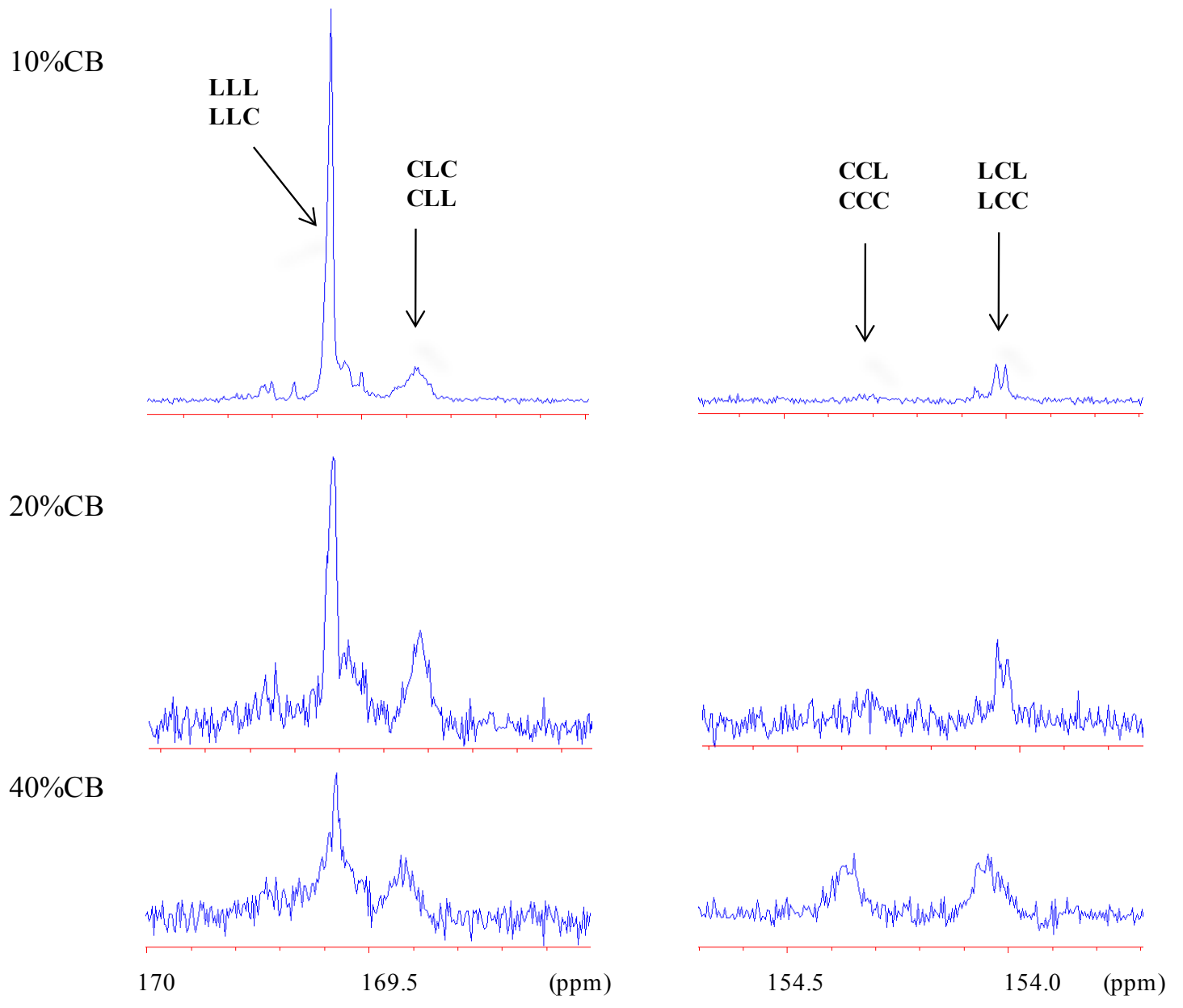

Figure 4-8. $\quad{ }^{13} \mathrm{C}$ NMR spectra comparative plot for 10,20 and 40 mol\% carbonate content demonstrating decrease in LLL sequence and increase in $\mathrm{CCC}$ sequence with increasing carbonate content. 


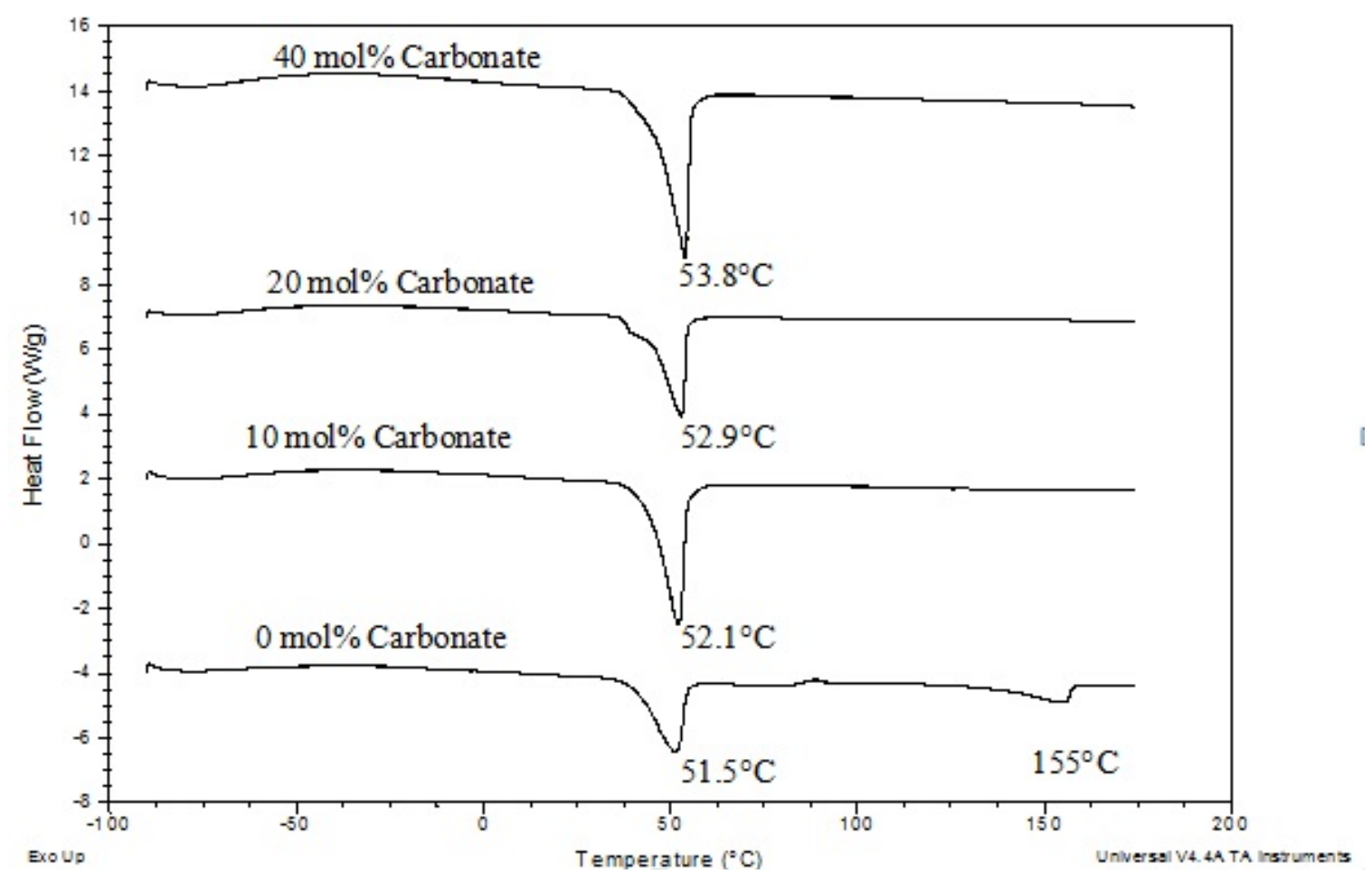

Figure 4-9. DSC thermograms of PEG-b-PLLA and PEG-b-P(CB-co-LA) copolymers. 


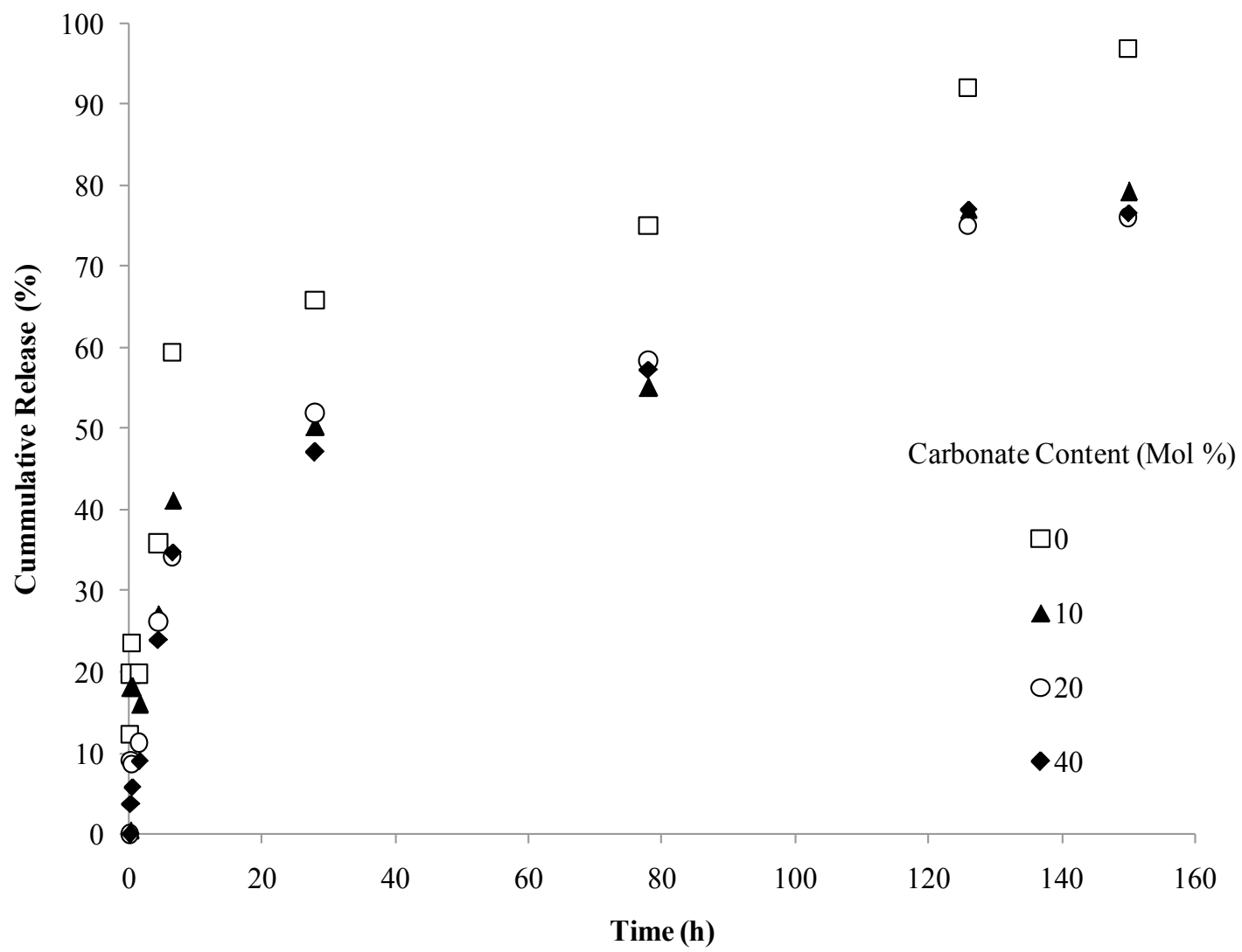

Figure 4-10. Effect of carbonate content on bicalutamide release from PEG-bPLLA and PEG-b-P(CB-co-LA) micelles.

Release experiments were performed in triplicate in $\mathrm{PBS}$ at $37^{\circ} \mathrm{C}$ and $100 \mathrm{rpm}$. 
this study had a GPC molecular weight of $5200 \mathrm{~g} \mathrm{~mol}^{-1}$ to ensure that all copolymers used in the release study had similar molecular weights.

Evaluation of in vitro cytotoxicity

To determine inhibitory effect of bicalutamide-loaded micelles formulated with PEG-b-P(CB-co-LA) copolymer on cell proliferation, the cytotoxicity of free bicalutamide dissolved in methanol and that of bicalutamide formulated in micelles was evaluated in LNCaP human prostate cancer cell line for $48 \mathrm{~h}$. From Figure 4-11, micelleformulated drug showed significant inhibition of $\mathrm{LNCaP}$ cell growth in a dose-dependent manner, which was similar to that obtained using free drug. The $\mathrm{IC}_{50}$ of bicalutamideloaded micelles was $74 \mu \mathrm{M}$ while that of free drug was $80 \mu \mathrm{M}$. However, no direct comparison can be made since we had to dissolve free drug in methanol, whereas we prepared micelles in PBS.

\section{Discussion}

In spite of the numerous advantages associated with polymeric micelles as drug delivery systems, their application as therapeutics has been limited by low loadings and stability. The equilibrium partitioning of a hydrophobic drug into the hydrophobic core of polymeric micelles at a specific temperature is governed by the molar free energy of the drug, which depends on the mixing entropy and the enthalpy interaction between the drug and the polymer, as well as the amount of pressure-volume work required in putting a drug molecule into the lipophilic core [232]. It has been shown for polymeric micelles that lowering the enthalpy interaction contribution of the free energy improves drug loading. This may be achieved by carefully selecting the core-forming block such that its solubility parameters and that of the drug are essentially of the same order, indicating superior drug-polymer compatibility. The Flory-Huggins interaction parameter $\left(\chi_{\mathrm{FH}}\right)$ is a good measure of the compatibility between the core-forming block of the copolymer and the drug, with lower values pointing to enhanced compatibility and superior loading.

The purpose of this study was to increase bicalutamide loading into micelles by designing polymers based on improved drug-polymer solubility determined using the Flory-Huggins interaction parameter. Inclusion of carbonate monomer which contains a bulky phenyl ring resulted in decreased $\chi_{\mathrm{FH}}$ values. In the strictest sense, $\chi_{\mathrm{FH}}$ values above the 0.5 critical value allude to immiscibility between drug and polymer since phase separation of the polymer-drug mixture commences, while $\chi_{\mathrm{FH}}$ values below 0.5 suggest that the drug and polymer are soluble in each other. The Flory-Huggins theory is based on the assumption that the orientation of molecules within the polymer-drug system is completely random and that upon mixing the polymer and drug no specific interactions are created or destroyed. However, since these assumptions are not entirely true for our system, the $\chi_{\mathrm{FH}}$ values obtained was merely used as a guide in predicting enhanced compatibility in relative terms based on the observed trend of lower $\chi_{\mathrm{FH}}$ values. It also provided a reasonable estimate of the closeness of the solubility parameters of 


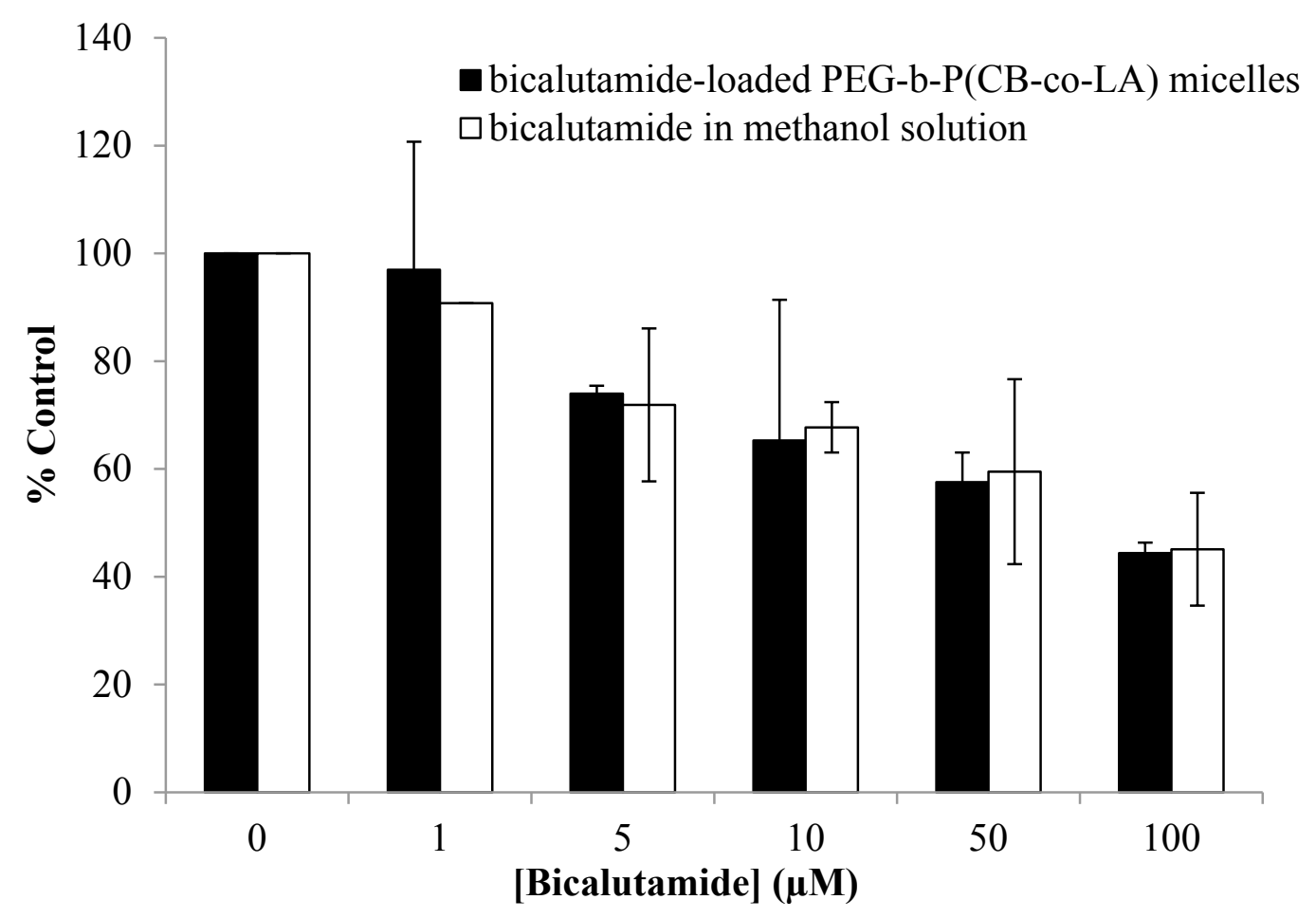

Figure 4-11. Effect of free and PEG-b-P(CB-co-LA) micellar formulation of bicalutamide on inhibition of LNCaP cell proliferation $48 \mathrm{~h}$ post treatment.

Cell viability was estimated by MTT assay and expressed as percent of $1 \%$ methanol or blank micelle controls. All data represent the mean \pm S.D. of triplicates. 
$\mathrm{P}(\mathrm{CB}-\mathrm{co}-\mathrm{LA})$ hydrophobic core and that of bicalutamide in the Hansen partial solubility three dimensional space compared to PLLA and bicalutamide.

A two-step reaction from 2,2-bis(hydroxymethyl)propionic acid was used to obtain the cyclic carbonate monomer (5-methyl-5-benzyloxycarbonyl-1,3-dioxane-2-one) which was subsequently employed in the synthesis of PEG-b-P(CB-co-LA) copolymer (Figure 4-1). All copolymers were obtained through ring opening polymerization of PEG, lactide and carbonate. Since our objective was to vary hydrophobic group to increase drug loading into micelles, we did not vary the molecular of PEG in our copolymers to determine the effect of PEG molecular weight on micelle number and diameter.

Various synthetic schemes have been reported for synthesizing 5-methyl-5benzyloxycarbonyl-1,3-dioxane-2-one [231, 233-235]. However, we employed the method described by Pratt et. al. since the yield of the cyclic carbonate monomer is higher for this method. The yield of the carbonate monomer $(95 \%)$ we obtained was similar to that obtained by Pratt et. al. [231] and our characterization data matched the literature [234, 236]. The ring opening polymerization of Lactide (LA) and the carbonate monomer was performed in bulk at $130^{\circ} \mathrm{C}$ with $\mathrm{Sn}(\mathrm{Oct})_{2}$ as catalyst $(10 \mathrm{~mol} \%$ relative to $\mathrm{mPEG}$ ) (Figure 4-1). We chose $\mathrm{Sn}(\mathrm{Oct})_{2}$ as a catalyst since it is FDA approved and $\mathrm{Sn}(\mathrm{Oct})_{2}$-catalyzed polymerization does not result in racemization of lactide at temperatures below $200^{\circ} \mathrm{C}$ [237]. Also, $\mathrm{Sn}(\mathrm{Oct})_{2}$ is a strong transesterification agent yielding copolymers with a randomized microstructure. Guan and coworkers reported synthesizing and characterizing a similar copolymer, however, they used $\mathrm{Et}_{2} \mathrm{Zn}$ as the catalyst and a reaction temperature of $100^{\circ} \mathrm{C}$ [235]. While a $7 \mathrm{~h}$ reaction time was sufficient for complete polymerization of PEG-b-PLLA, it resulted in incomplete polymerization of PEG-b-P(CB-co-LA) copolymers. A reaction time of $24 \mathrm{~h}$ was used for PEG-b-P(CB-co-LA) copolymers since complete polymerization was observed after $24 \mathrm{~h}$. The observed lower DP of P(CB-co-LA) core compared to the predicted DP suggests a lowering of the carbonate and lactide monomer reactivity with increasing carbonate content. From ${ }^{1} \mathrm{H}$ NMR spectroscopy, the carbonate monomer was observed to have higher reactivity compared to lactide. Also, increasing carbonate content from 10 to 40 mol $\%$ resulted in lower reactivity for both carbonate and lactide monomers: carbonate reactivity decreased from $87 \%$ to $61 \%$ while lactide reactivity decreased from $66 \%$ to $24 \%$. Overall, the molecular weight decreases while the molecular weight distribution increases with increasing carbonate content. This may be due to progressively higher levels of intra-(backbiting) and intermolecular (redistribution) transesterification side reactions resulting in the formation of cyclic oligomers as the carbonate content increases.

PEG-b-P(CB-co-LA) copolymers self assembled into micelles with a core-shell structure (Figure 4-6). The average diameter of micelles formulated from PEG-b-P(CBco-LA) copolymers was in the range of 99 to $123 \mathrm{~nm}$ and appeared to be independent of carbonate content but correlated well with molecular weight. The introduction of carbonate monomer enhanced bicalutamide loading (Table 4-3), which confirmed our prediction based on the Flory-Huggins interaction parameter of PEG-b-P(CB-co-LA) 
having superior bicalutamide loading to PEG-b-PLLA copolymer. However, this increase was not linear with carbonate content and seemed to peak at the $20 \mathrm{~mol} \%$ content where a four-fold increase in bicalutamide loading was observed compared to PEG-b-PLLA copolymer. We also observed that while the drug loading (as defined by equation 3 ) in $\mathrm{PEG}_{114}-\mathrm{b}-\mathrm{P}\left(\mathrm{CB}_{5}-\mathrm{co}-\mathrm{LA}_{32}\right)$ copolymer was approximately three fold higher than that of $\mathrm{PEG}_{114}-\mathrm{b}-\mathrm{P}\left(\mathrm{CB}_{9}-\mathrm{co}-\mathrm{LA}_{5}\right)$, the drug loading on a molar basis in the hydrophobic core was about the same. Hence, the rather low drug loading observed for the $40 \mathrm{~mol} \%$ carbonate series may be due to a couple of factors: (1) its relatively smaller molecular weight compared to the 10 and $20 \mathrm{~mol} \%$ carbonate series and (2) the decreased PEG-b-P(CB-coLA) copolymer solubility with increasing carbonate content. As the feed carbonate content increases, the reactivity of both the lactide and carbonate decreases. Lactide reactivity decreases from $86 \%$ to $24 \%$ while carbonate reactivity decreases from $82 \%$ to $64 \%$ for $0 \mathrm{~mol} \%$ to $40 \mathrm{~mol} \%$ carbonate content. This coupled with the polymer degradation effect associated with intramolecular transesterifaction leads to the decreased molecular weight observed with increasing carbonate content. It is well known, that drug loading is related to the molecular weight of the hydrophobic core. Large hydrophobic blocks form large micelle cores and require less amount of work to put a molecule of drug into the hydrophobic core due to relatively lower internal pressure. Drug loading is also influenced by the interaction between the drug and the copolymer as well as the solubility of the copolymer. As the carbonate content increases the interaction between bicalutamide and the copolymer increases, however, the solubility of the copolymer decreases. Hence, the trade-off between drug-polymer interaction and polymer solubility influences the extent of drug loading. At carbonate content up to $20 \mathrm{~mol} \%$ the effect of drug-polymer interaction predominates resulting in the observed improved drug loading, while at $40 \mathrm{~mol} \%$ carbonate content the effect of decreased copolymer solubility dominates contributing to the observed reduction in drug loading. As such, the PEG-b$\mathrm{P}(\mathrm{CB}-\mathrm{co}-\mathrm{LA}) 20 \mathrm{~mol} \%$ carbonate series exhibit optimum drug loading. It is worth mentioning that we also synthesized PEG-b-Polycarbonate (PEG-b-PCB) using the same polymerization conditions for PEG-b-P (CB-co-LA) copolymer. However, the solubility of this polymer in tetrahydrofuran (THF) and commonly used organic solvents was poor; making characterization using GPC and NMR difficult. From Table 4-2, drug solubility appears to correlate with the CMC. Among the series of copolymers, $0 \mathrm{~mol} \%$ carbonate has the highest CMC value of $32.95 \times 10^{-7} \mathrm{~mol}^{-1}$ and the lowest drug loading of $1.0 \% \pm$ 0.31 , while the $20 \mathrm{~mol} \%$ carbonate series has the lowest $\mathrm{CMC}$ value $3.13 \times 10^{-7} \mathrm{~mol}^{-1}$ and the highest drug loading $4.1 \% \pm 0.23$. The decrease in the $\mathrm{CMC}$ upon inclusion of the carbonate monomer clearly demonstrates that they have higher thermodynamic stability compared to PEG-b-PLLA. A high thermodynamic stability is desirable for in vivo stability of polymeric micellar systems.

Sequence distribution of random copolymers can influence fundamental polymer properties such as solubility, mechanical and thermal properties. The observed decrease in $\mathrm{L}-\mathrm{L}$ sequence and increase in $\mathrm{C}-\mathrm{C}$ sequence as carbonate content increases can be used to elucidate the behavior of the PEG-b-P(CB-co-LA) copolymers. When the ratio of lactide to carbonate monomer is small, the lactide segments in the random copolymer are sparsely distributed and have a lower probability of interacting with the ethylene oxide segments since the lactide is buried in the carbonate segments hence preventing 
intermolecular interactions. This may explain the observed sharp decrease in lactide reactivity (from 66 to 24\%). Conversely, at high lactide to carbonate ratios, lactide segments tend to undergo intramolecular transesterification and lead to smaller molecular weight polymers due degradation. Hence, these two competing phenomena affect polymer molecular weight depending on which factor is more dominant and the optimum molecular weight is obtained when they are balanced. In the present study, the $20 \mathrm{~mol} \%$ carbonate series exhibited optimum molecular weight which translated into superior drug loading and thermodynamic stability.

Thermal analysis of the copolymers by differential scanning calorimetry confirmed the semicrystalline nature of PLLA. The two endotherm peaks observed for PLLA reflect the melting temperature of PEG $\left(\mathrm{T}_{\mathrm{m}}=51.5^{\circ} \mathrm{C}\right)$ and melting temperature of PLLA $\left(\mathrm{T}_{\mathrm{m}}=155^{\circ} \mathrm{C}\right)$, respectively. Also the exotherm peak occurring at $88.5^{\circ} \mathrm{C}$ was also observed which may signify the onset of cold crystallization. More importantly, the data revealed that the introduction of the carbonate monomer into the poly (L-lactide) chain resulted in a reduction in crystallinity as demonstrated by the absence of the cold crystallization and melting peaks in the copolymers. These results suggest that the carbonate monomer randomly incorporated into the PLLA chain and resulted in a shortening of lactide sequences thereby hindering the crystallization process. Our data is consistent with the literature and other groups have reported similar trends $[235,238]$.

In vitro release studies clearly showed the introduction of the carbonate monomer to result in better sustained release of bicalutamide from the micelle core compared to PEG-b-PLLA. The improved sustained release of the carbonate modified copolymers can be attributed to better compatibility between bicalutamide and the hydrophobic block of the copolymer as well as they being more amorphous compared to PEG-b-PLLA. Both PEG-b-PLLA and PEG-b-P(CB-co-LA) copolymers exhibited fast release during the first $6 \mathrm{~h}$. This burst release is assumed to be due to drug trapped with the PEG corona and at the corona-core interface. Increasing carbonate content from 10 to $40 \mathrm{~mol} \%$ does not appear to alter the release profile of bicalutamide over the $150 \mathrm{~h}$ period studied. The release behavior of hydrophobic drugs from spherical micelles is known to depend on the properties of the hydrophobic core. The inhibitory effect of bicalutamide-loaded micelles formulated with PEG-b-P(CB-co-LA) copolymer on cell proliferation was comparable to free drug solubilized in methanol in LNCaP cells. This result suggests that bicalutamideloaded micelles formulated with PEG-b-P(CB-co-LA) copolymer did not diminish the inhibitory effect of bicalutamide on cell proliferation since the PEG-b-P(CB-co-LA) copolymers themselves were not toxic in LNCaP cells. Although methanol is a toxic reagent, its intrinsic toxicity does not significantly alter the results since an equivalent of $1 \% \mathrm{v} / \mathrm{v}$ methanol was used with appropriate controls. Moreover, we have previously used dimethyl sulfoxide (DMSO), which was found to kill tumor cells.

\section{Conclusions}

We established that chemical tailoring of the PEG-b-PLLA micelle core through the introduction of carbonate moieties enhanced the solubilization of the highly lipophilic 
drug bicalutamide. This result was consistent with predictions made based on the FloryHuggins interaction parameters computed for polymer/bicalutamide pairs using the group contribution method. Bicalutamide loading in PEG-b-P(CB-co-LA) micelles was four times better than in PEG-b-PLLA. PEG-b-P(CB-co-LA) copolymers were also shown to significantly solubilize bicalutamide as aqueous micellar solutions had drug concentrations approximately 800 -fold higher than the saturated solubility of bicalutamide in water. In water, PEG-b-P(CB-co-LA) copolymers formed spherical micelles with $\mathrm{CMC}$ values up to 10 times lower than PEG-b-PLLA copolymers (i.e., 0.31 $\mu \mathrm{M}$ for $20 \mathrm{~mol} \%$ carbonate and $3.3 \mu \mathrm{M}$ for $0 \mathrm{~mol} \%$ carbonate), indicating that introduction of carbonate monomer results in copolymers which are more thermodynamically favored for self-assembly and exhibit better thermodynamic stability. Finally, PEG-b-P(CB-co-LA) copolymers was found to result in slower bicalutamide release compared to PEG-b-PLLA copolymers. Even though the inhibitory effect of bicalutamide-loaded PEG-b-P(CB-co-LA) micelles on LNCaP cell proliferation was similar to free drug these results are quite significant since drug dissolved in methanol would not be suitable for clinical application. In conclusion, these studies highlight the effect of chemically modifying the PEG-b-PLLA micelle core with a carbonate monomer on drug loading, release and stability and demonstrate their potential use as drug delivery vehicles. In vivo testing of bicalutamide loaded PEG-b-P(CB-co-LA) micelles for tumor treatment is ongoing and will be reported in a separate paper. 


\section{CHAPTER 5. COMBINATION THERAPY OF ANTIANDROGEN AND XIAP INHIBITOR FOR TREATING ADVANCED PROSTATE CANCER*}

\section{Introduction}

Prostate cancer is the most frequently diagnosed neoplasm and the second leading cause of cancer mortality affecting men in the United States [239]. Nearly all prostate carcinomas are initially androgen dependent [240]. Hence, the disease is classified as hormone-dependent or hormone-refractory depending on the sensitivity of the androgen receptor (AR) to androgens. To date, androgen ablation therapy using antiandrogens remains the gold standard for treating hormone-dependent prostate cancer and is effective in approximately $70 \%$ of patients [241]. In spite of its initial efficacy, androgen ablation results in remission lasting two to three years after which the disease recurs in its more aggressive hormone refractory form for which there is no cure [242, 243]. Consequently, there is an urgent need for novel therapeutic strategies for treating hormone refractory prostate cancer.

We have previously shown that combination therapy targeting AR and XIAP using bicalutamide and embelin is a promising approach to treat prostate cancer [11]. However, first generation antiandrogens such as bicalutamide exhibit agonist characteristics upon prolonged treatment due to increased AR gene amplification [244, 245]. Therefore, we hypothesized new antiandrogens more effective than bicalutamide will be beneficial in treating advanced prostate cancer when combined with XIAP modulation. Hence, our group synthesized a novel antiandrogen ((S)-N-(4-cyano-3(trifluoromethyl) phenyl)-3-((4-cyanophenyl)(methyl)amino)-2-hydroxy-2-

methylpropanamide) (CBDIV17)) using bicalutamide as a starting chemical scaffold due to its relatively high AR binding affinity and selectivity (Figure 5-1A).

Systemic delivery of anticancer agents to solid tumors is still a challenge. Aside being toxic, current solubilizing agents such as Cremophor ${ }^{\circledR}$ EL and DMSO contribute to erratic extravasation to tumors leading to sub-optimal therapeutic effects [246]. Polymeric micelles are an elegant way to improve the solubility, stability and extravasation of drugs to tumors thereby enhancing therapeutic potential $[11,170,246-$ 248]. They self-assemble from amphiphilic diblocks and are typically nanosized, spherical structures with a hydrophobic core which serves as a cargo space for hydrophobic drugs. We recently engineered and synthesized polyethylene glycol-b-poly (carbonate-co-lactide) (PEG-b-p(CB-co-LA)) copolymers with excellent drug loading and stability for optimal delivery of bicalutamide [170], and we will explore the use of this copolymer to formulate CBDIV17 and embelin in this work.

*Danquah M, Duke CB, 3rd, Patil R, Miller DD, Mahato RI. Combination Therapy of Antiandrogen and XIAP Inhibitor for Treating Advanced Prostate Cancer. Pharm Res. 2012; 29: 2079-91. 
A

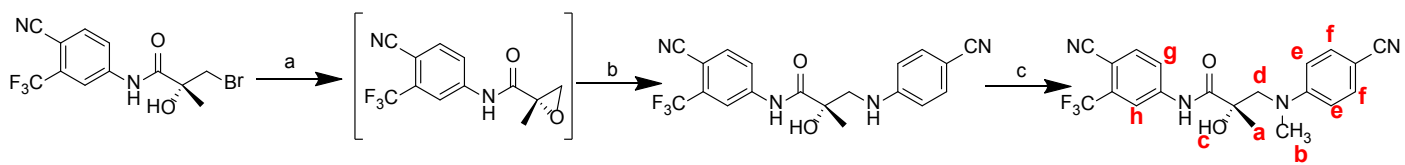

a. $\mathrm{K}_{2} \mathrm{CO}_{3}, 2$-propanol, reflux $2 \mathrm{~h}$

b. 1,1,3,3,3-hexafluoro-2-propanol, 4-amino-benzonitrile, $24 \mathrm{~h}$

c. Iodomethane, Hünig's base, $110^{\circ} \mathrm{C}, 3 \mathrm{~d}$

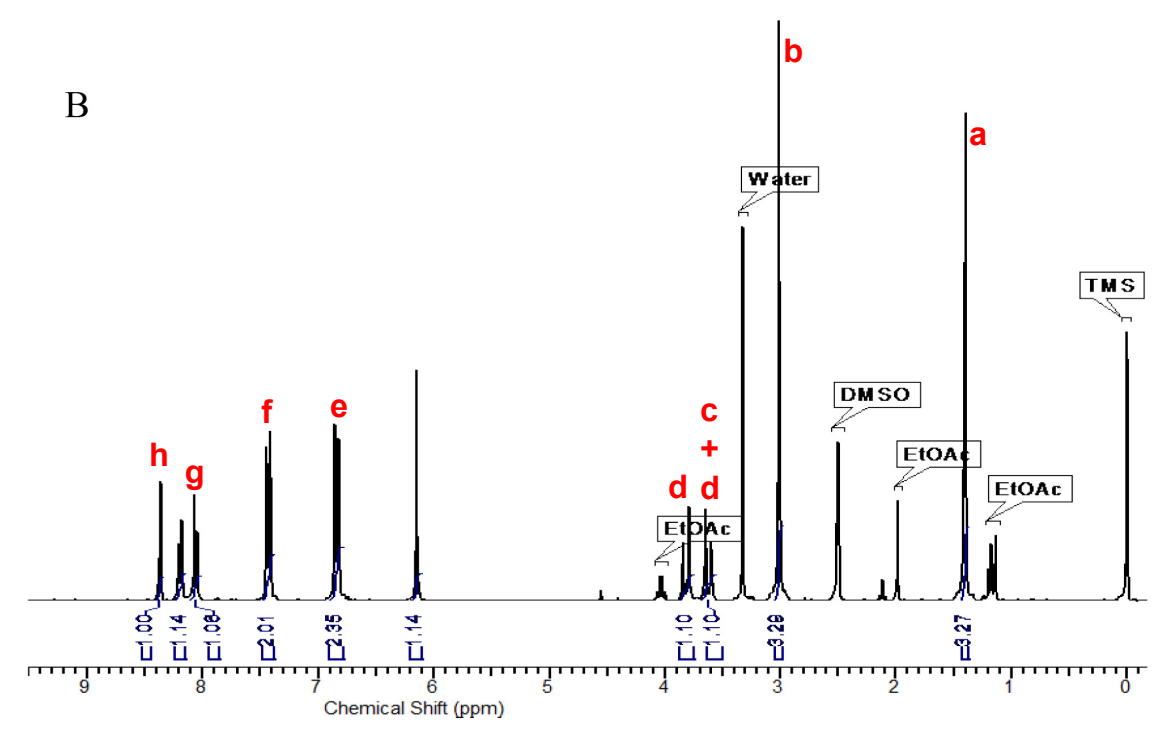

Figure 5-1. Synthesis and characterization of (S)-N-(4-cyano-3(trifluoromethyl)phenyl)-3-((4-cyanophenyl)(methyl)amino)-2-hydroxy-2methylpropanamide) (CBDIV17).

(A) Synthesis scheme of CBDIV17 and (B) ${ }^{1} \mathrm{H}$ NMR spectrum of CBDIV17. 
The focus of the present study is three fold: First to demonstrate the superiority of CBDIV17 in treating prostate cancer compared to bicalutamide. Second, to evaluate two XIAP inhibitors: embelin and 2,5-dihydroxy-3-(2-[4-(2-m-tolyl-ethyl)-phenyl]-ethyl)$[1,4]$ benzoquinone (embelin- $6 \mathrm{~g}$ ) to determine the more appropriate compound to combine with CBDIV17. Third, to show that combination of CBDIV17 and chosen XIAP inhibitor is more potent than control or monotherapy in inhibiting cell growth and inducing apoptosis in vitro as well as in regressing prostate cancer tumors in xenograft mouse model.

\section{Materials and Methods}

Materials

SYBR Green real-time RT-PCR master mix and reverse transcription reagents were purchased from Applied Biosystems (Foster City, CA). Human total XIAP ELISA kit was purchased from R\&D Systems (Minneapolis, MN). Primary and secondary antibodies were purchased from Abcam (Cambridge, MA). All other reagents were obtained from Sigma-Aldrich (St. Louis, MO) unless otherwise stated and were used as received.

\section{Synthesis of CBDIV17}

CBDIV17 was synthesized by treating 3-Bromo-2-hydroxy-N-(4-isocyano-3trifluoromethyl-phenyl)-2-methyl-propionamide (bromide $\mathrm{N}$ ) with $\mathrm{K}_{2} \mathrm{CO}_{3}$ in 2-propanol at reflux for $2 \mathrm{~h}$ before filtering the solution. The reaction mixture was concentrated to a paste, dissolved in EtOAc, and washed with water before drying using $\mathrm{Na}_{2} \mathrm{SO}_{4}$ and concentrating to a resin. The epoxide intermediate was then treated with 4aminobenzonitrile in 1, 1, 1, 3, 3, 3-hexafluoro-2-propanol at $100{ }^{\circ} \mathrm{C}$ overnight. Flash chromatography on silica gel (EtOAc/Hexanes 20-100\%) afforded (S)-N-(4-cyano-3(trifluoromethyl)phenyl)-3-((4-cyanophenylamino)-2-hydroxy-2-methylpropanamide), which was treated with MeI and Hünig's base in a pressure vessel for 3 days at $110^{\circ} \mathrm{C}$. Flash chromatography on silica gel (EtOAc/Hexanes 20-100\%) afforded CBDIV17.

In vitro cell viability assays

LNCaP and C4-2 cells (American Type Culture Collection) were incubated in RPMI 1640 media, $10 \%$ fetal bovine serum and $1 \%$ antibiotic-antimycotic at $37^{\circ} \mathrm{C}$ in humidified environment of 5\% $\mathrm{CO}_{2}$ and subcultured every 3-4 days to maintain exponential growth. Cells were seeded in 96-well plates at a density of $1 \times 10^{4}$ viable cells/well and incubated for $48 \mathrm{~h}$. The cells were exposed to antiandrogen or XIAP inhibitor at various concentrations for specified time periods. At the end of treatment, 20 $\mu 1$ of MTT $(5 \mathrm{mg} / \mathrm{ml})$ was added to each well, incubated for 3-4 h and analyzed at a test wavelength of $560 \mathrm{~nm}$. Cell viability was expressed as a percentage of the intensity of 
controls. LNCaP cells are androgen dependent and non-bone metastatic prostate cancer cells with low tumorigenicity. In contrast, C4-2 cells (a subline of LNCaP) are androgen independent and more tumorigenic compared to LNCaP cells. These cells lines were selected as in vitro models since they both express the androgen receptor and possess a threonine to alanine mutation of amino acid 877 (T877A) in the androgen receptor [249, 250]. Furthermore, $\mathrm{LNCaP}$ represents hormone dependent prostate cancer whereas C4-2 is a model for advanced prostate cancer.

\section{Real-time RT-PCR}

RNA extraction and real-time RT-PCR was performed as previously described [11]. Primers are as follows: AR: forward, 5'-AGC CAT TGA GCC AGG TGT AG-3'; reverse, 5'-CGT GTA AGT TGC GGA AGC C-3'. PSA: forward, 5'-GTG GGT CCC GGT TGT CT-3'; reverse, 5'-AGCCCAGCTCCCTGTCT-3'. XIAP: forward, 5'-TGT TTC AGC ATC AAC ACT GGC ACG-3'; reverse, 5'- GCA TGA CAA CTA AAG CAC CGG AC-3'. Cyclin D1: forward, 5'- GAT GCC AAC CTC CTC AAC GAC -3'; reverse, 5'- CTC CTC GCA CTT CTG TTC CTC -3'.

Western blot analysis

Cells were treated with embelin and embelin-6g for $96 \mathrm{~h}$. Subsequently, cells were lysed using RIPA buffer (Sigma-Aldrich, St. Louis, MO) and protein concentration measured with bicinchoninic acid (BCA) protein assay kit (Pierce, Rockford, IL). The lysate was boiled for 5 mins, subjected to a 15\% SDS-PAGE and transferred to a PVDF membrane using iBlot ${ }^{\mathrm{TM}}$ system (Invitrogen, Carlsbad, CA). Membranes were blocked with $3 \%$ BSA in $1 \mathrm{x}$ tris buffered saline (TBS) at room temperature for $1 \mathrm{~h}$ and then incubated with primary antibodies at $4^{\circ} \mathrm{C}$ overnight, followed by incubation with secondary antibody conjugated with horseradish peroxidase (HRP) at room temperature for $1 \mathrm{~h}$. The signal of target proteins was detected using Immun-Star HRP chemiluminescent kit (BioRad, Hercules, CA) or Li-COR Odyssey ${ }^{\circledR}$ infrared imaging system (Li-COR, Lincoln, NE).

XIAP detection using ELISA

C4-2 cells were treated with embelin or embelin-6g for $48 \mathrm{~h}$. Cells were lysed and XIAP concentration detected using Human XIAP ELISA kit as described in the manufacturer's protocol.

Apoptosis and cell cycle analysis

Following treatment with $25 \mu \mathrm{M}$ antiandrogen for $72 \mathrm{~h}$, cells were trypsinized and fixed in $70 \%$ ice-cold ethanol and washed with PBS. Samples were resuspended in $500 \mu 1$ 
of propidium iodide solution containing RNAse A (BD Pharmingen, San Diego, CA) for 15 min at room temperature. Relative DNA content per cell was acquired by measuring DNA fluorescence using flow cytometry and subsequent analysis performed with Modfit program.

Confocal microscopy

Confocal microscopy was used to elucidate the effect of XIAP inhibitor on DHT stimulated nuclear translocation of AR. Briefly, LNCaP and C4-2 cells were cultured in chamber slides in RPMI media and treated with XIAP inhibitor $(5 \mu \mathrm{M})$ or DMSO for $3 \mathrm{~h}$ and then incubated with DHT $(1 \mathrm{nmol} / \mathrm{L})$ for an additional $3 \mathrm{~h}$. Cells were fixed in methanol at room temperature for $10 \mathrm{~min}$, washed with PBS, and incubated with PBS containing $10 \%(\mathrm{w} / \mathrm{v})$ rabbit serum for $2 \mathrm{~h}$ at room temperature. Afterwards, the cells were treated with anti-AR antibody (1:100 in PBS buffer) $4{ }^{\circ} \mathrm{C}$ overnight. Cells were then washed with PBS and incubated with FITC-conjugated secondary antibody for $1 \mathrm{~h}$ at room temperature followed by counterstaining with DAPI. Slides were examined under a Zeiss LSM-710 confocal microscope at $63 \times$ objective lens magnification and data processed using a ZEN 2009 LE software.

Preparation and characterization of micelles

The film sonication method was used for loading drug into the core of PEG-b$\mathrm{p}$ (CB-co-LA) micelles at a theoretical drug loading of 5\%. Briefly, $5 \mathrm{mg}$ of drug and 95 $\mathrm{mg}$ of PEG-b-p(CB-co-LA) was dissolved in $5 \mathrm{ml}$ methanol. The mixture was allowed to stir for $5 \mathrm{~min}$ and the solvent evaporated. The resulting film was hydrated and sonicated for 7 min using a Misonix ultrasonic liquid processor (Farmingdale, NY) with an output power of $25 \mathrm{~W}$. The resultant formulation was then centrifuged at 5,000 rpm for $10 \mathrm{~min}$ to separate micelles from residual free drug. Subsequently, the supernatant was filtered using a $0.22 \mu \mathrm{m}$ nylon filter.

In vivo efficacy of CBDIV17 and embelin-loaded micelles in mouse xenografts

All animal experiments were performed in a humane manner in accordance with NIH animal use guidelines and the protocol approved by the Animal Care and Use Committee (ACUC) at the University of Tennessee Health Science Center. Xenograft flank tumors were induced in 6 week old male athymic nu/nu mice (Jackson Laboratory, Bar Harbor, ME) by subcutaneous injection of 3 million C4-2 cells suspended in 1:1 media and matrigel. When tumors reached approximately $50 \mathrm{~mm}^{3}$, mice were randomized into four groups of 7 mice, minimizing weight and tumor size differences. Each group was treated with intratumoral injection of blank micelles or drug-loaded micelles. Tumors were measured with a caliper prior to each injection, and their volumes calculated using the formula of (width ${ }^{2} \mathrm{x}$ length) $/ 2$. 


\section{Results}

Synthesis and characterization of CBDIV17

CBDIV17 was synthesized as shown in Figure 5-1A and characterized by ESI and high-resolution mass spectrometries, ${ }^{1} \mathrm{H}$ NMR (Figure 5-1B) and ${ }^{13} \mathrm{C}$ NMR. MS (ESI): calculated for $\mathrm{C}_{20} \mathrm{H}_{17} \mathrm{~F}_{3} \mathrm{~N}_{4} \mathrm{O}_{2} 402.1$, found 400.9 [M - H]. HRMS calculated for $\mathrm{C}_{20} \mathrm{H}_{17} \mathrm{~F}_{3} \mathrm{~N}_{4} \mathrm{O}_{2} 403.13818$ found 403.13696. ${ }^{1} \mathrm{H}$ NMR (300 MHz, chloroform-d) 10.42 $10.51(\mathrm{~m}, 1 \mathrm{H}), 8.33-8.41(\mathrm{~m}, 1 \mathrm{H}), 8.14-8.24(\mathrm{~m}, 1 \mathrm{H}), 8.06(\mathrm{~d}, J=8.54 \mathrm{~Hz}, 1 \mathrm{H}), 7.43$ $(\mathrm{d}, J=9.16 \mathrm{~Hz}, 2 \mathrm{H}), 6.84(\mathrm{~d}, J=9.16 \mathrm{~Hz}, 2 \mathrm{H}), 6.15(\mathrm{~s}, 1 \mathrm{H}), 3.81(\mathrm{~d}, J=15.26 \mathrm{~Hz}, 1 \mathrm{H})$, $3.62(\mathrm{~d}, J=14.95 \mathrm{~Hz}, 1 \mathrm{H}), 3.01(\mathrm{~s}, 3 \mathrm{H}), 1.37-1.43(\mathrm{~m}, 3 \mathrm{H}) \mathrm{ppm}$ (Figure 5-1) ${ }^{13} \mathrm{C}$ NMR $(101 \mathrm{MHz}$, DMSO-d 6 ) $\delta 175.4,152.3,142.9,136.1,132.7,131.3(\mathrm{q}, J=31.5 \mathrm{~Hz}), 122.8$, $122.4(\mathrm{q}, J=276.6 \mathrm{~Hz}), 120.2,117.5(\mathrm{q}, J=5.1 \mathrm{~Hz}), 115.7,112.0,101.9,95.9,76.7$, 59.3, 39.7, 39.4 (spt, DMSO), 23.7, 0.0 (s, TMS) ppm.

CBDIV17 and bicalutamide inhibit LNCaP and C4-2 cell growth

We first assessed anticancer activity of CBDIV17 and bicalutamide in LNCaP and C4-2 cells. Both cells are AR positive and possess the T877A androgen receptor mutant. As shown in Figure 5-2A and $\mathbf{B}$, bicalutamide and CBDIV17 exhibited dosedependent anticancer activities in both cells when treated with $0,10,25$ and $50 \mu \mathrm{M}$ for 72 h. From our results, CBDIV17 was more potent than bicalutamide at each dose in both cell lines. $\mathrm{IC}_{50}$ for CBDIV17 was 12 and $21 \mu \mathrm{M}$ in LNCaP and C4-2 cells, respectively. In contrast, bicalutamide had an $\mathrm{IC}_{50}$ of $46 \mu \mathrm{M}$ in $\mathrm{LNCaP}$ with only a modest anticancer effect being observed at $50 \mu \mathrm{M}$ in C4-2 cells. Furthermore, a time course study using a drug concentration of $25 \mu \mathrm{M}$ for 24 and $72 \mathrm{~h}$ revealed the anticancer effect of bicalutamide and CBDIV17 to be time-dependent (Figure 5-2C). Our data suggests CBDIV17 displays superior anticancer activity in LNCaP cells even after a short exposure time. Additionally, there is a dramatic increase in inhibition of prostate cancer cell growth for CBDIV17 compared to bicalutamide when cell exposure to drug is increased from 24 to $72 \mathrm{~h}$.

We next examined the influence of androgens on the anticancer activity of CBDIV17 since it is expected to function as a putative anti-androgen. LNCaP cells were cultured in androgen-depleted media with 5\% charcoal-stripped serum (CSS). Cells were treated for 24 or 72 hours with or without $1 \mathrm{nM}$ of the synthetic androgen $5 \alpha$ Dihydrotestosterone (DHT) combined with DMSO (vehicle), CBDIV17 and bicalutamide (positive control). Our data suggests that treatment of cells with DHT stimulated cell growth (Figure 5-3). However, even in the presence of DHT, cell growth was inhibited when cells were treated with bicalutamide or CBDIV17, with CBDIV17 being more potent than bicalutamide. 

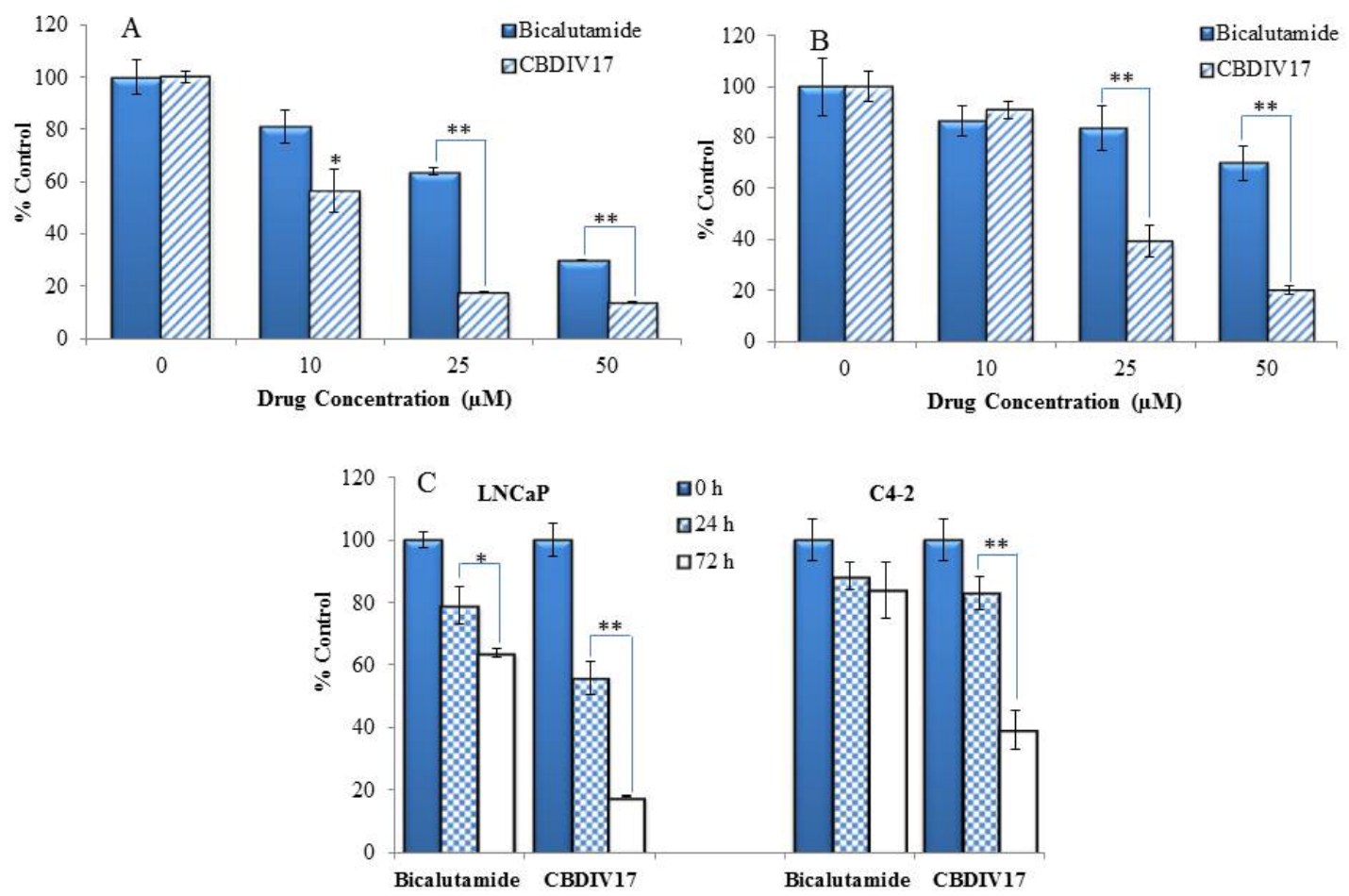

Figure 5-2. Anticancer effect of antiandrogen on prostate cancer cells.

(A and $\mathrm{B}$ ) $\mathrm{IC}_{50}$ of bicalutamide and CBDIV17 in $\mathrm{LNCaP}$ and $\mathrm{C} 4-2$ cells were calculated following treatment with $0,10,25$ and $50 \mu \mathrm{M}$ of drug for $72 \mathrm{~h}$ and cell viability determined by MTT assay. (C) Effect of antiandrogen with time was determined by exposing LNCaP and C4-2 cells to $25 \mu \mathrm{M}$ of drug for 24 and $72 \mathrm{~h}$. Cell viability was then determined by MTTassay. Results are represented as the mean \pm SD of triplicates. ${ }^{*} \mathrm{p}<$ $0.05 ; * * \mathrm{p}<0.01$ using student's unpaired t-test. 


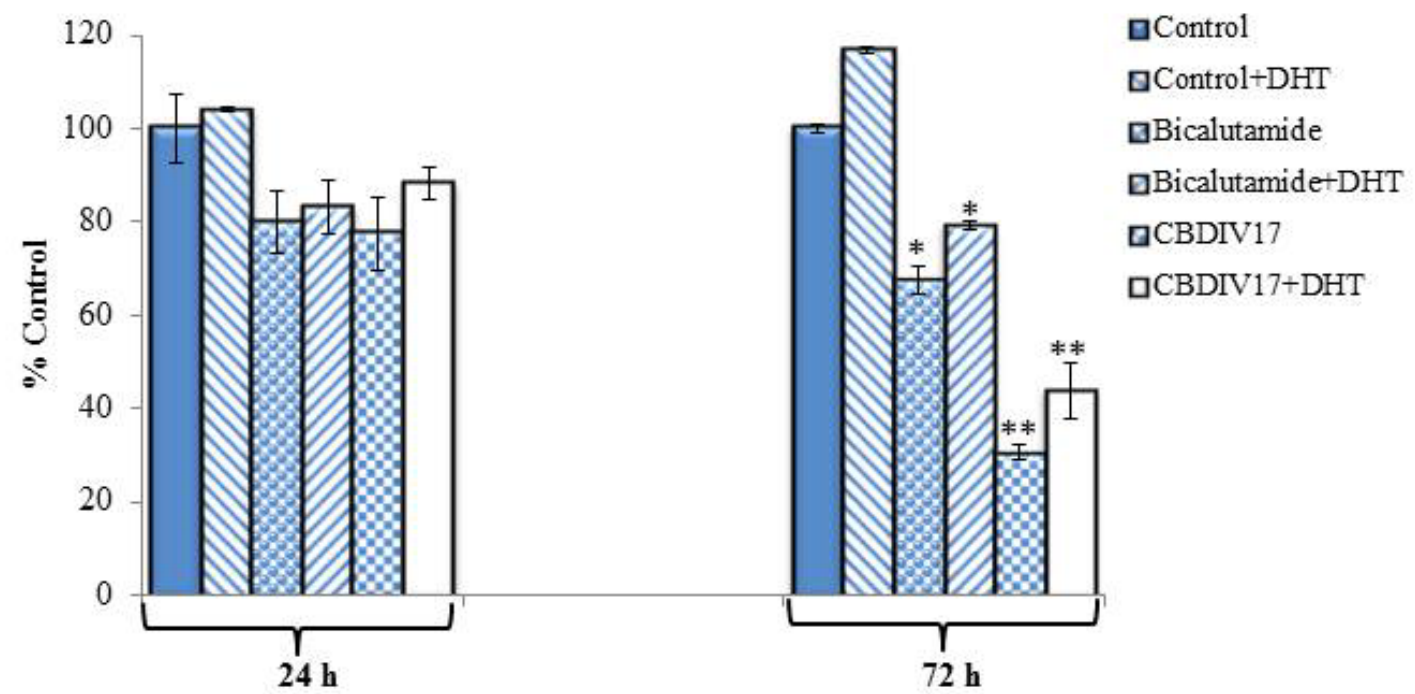

Figure 5-3. Effect of bicalutamide and CBDIV17 on cell proliferation in the presence of androgen.

LNCaP cells were cultured in androgen-depleted media with 5\% charcoal-stripped serum (CSS). Cells were treated for 24 or 72 hours with or without $1 \mathrm{nM}$ of the synthetic androgen $5 \alpha$-Dihydrotestosterone (DHT) combined with DMSO, bicalutamide and CBDIV17. Results are represented as the mean \pm SD of triplicates. ${ }^{*} \mathrm{p}<0.05$;

$* * \mathrm{p}<0.01$ using student's unpaired $\mathrm{t}$ - test. 
CBDIV 17 and bicalutamide induce apoptosis and alter cell cycle of LNCaP and C4-2 cells

The effect of bicalutamide and CBDIV17 on the cell cycle of LNCaP and C4-2 cells was determined following treatment with $25 \mu \mathrm{M}$ of these drugs for $72 \mathrm{~h}$. We observed a significant reduction in the percent of cells in $\mathrm{S}$ phase in both cell lines (Figure 5-4). In LNCaP cells, $27 \%$ of control cells were in the S phase, whereas $16 \%$ of bicalutamide treated cells and $6.5 \%$ of CBDIV17 treated cells were in the S phase. For C4-2 cells, $32.6 \%$ of control cells were in the S phase whilst $27.7 \%$ of bicalutamide treated cells and $10.7 \%$ of CBDIV17 treated cells were in the S phase. Furthermore, drug treatment in LNCaP cells did not result in significant decrease in percent of cells in the $\mathrm{G} 2 / \mathrm{M}$ phase. In contrast, percent of C4-2 cells in the $\mathrm{G} 2 / \mathrm{M}$ phase decreased from $16 \%$ in control cells to $8.9 \%$ in bicalutamide treated cells and to $4.8 \%$ in CBDIV17 treated cells.

Treatment with bicalutamide and CBDIV17 resulted in a significant increase in the percentage of cells in the $\mathrm{G} 0 / \mathrm{G} 1$ phase in both cell lines. LNCaP cell population in the $\mathrm{G} 0 / \mathrm{G} 1$ phase increased from $67 \%$ in control cells to $76 \%$ and $87 \%$ for bicalutamide and CBDIV17 treated cells, respectively. For C4-2 cells, percentage of cells in G0/G1 phase increased from $51.4 \%$ in control cells to $63.3 \%$ and $84.5 \%$ for bicalutamide and CBDIV17 treated cells, respectively. Finally, a significant increase in the sub-G1 phase (indicative of apoptosis) was observed upon administration of bicalutamide (13-fold) and CBDIV17 (18-fold) to LNCaP cells (Figure 5-4). In C4-2 cells, treatment with bicalutamide resulted in a 7-fold increase in sub-G1 whereas CBDIV17 led to a 10-fold increase in percentage of cells in subG1 phase. These data demonstrate that CBDIV17 was more potent than bicalutamide in altering cell cycle leading to G1 arrest, reducing DNA synthesis and inducing cell death.

Embelin-6g is more potent than embelin in repressing XIAP expression

After demonstrating that CBDIV17 is more potent than bicalutamide, we next evaluated the mechanism and therapeutic potential of embelin and embelin-6g (Figure 5-5A) to determine which XIAP inhibitor should be used in conjunction with CBDIV17 for combination therapy. Since embelin-6g is reported to be a more potent XIAP inhibitor than embelin [251], we examined its effect on XIAP expression. Our observations indicated that embelin-6g repressed XIAP mRNA expression by 2 -fold compared to embelin (Figure 5-5B). We also assessed the effect of these drugs on XIAP protein using ELISA. Embelin-6g more potently inhibited XIAP expression compared to embelin with the effect being almost twice that of embelin (Figure 5-5C).

Embelin and its derivative downregulate AR expression and repress AR mediated activity

In addition to elucidating the effect of embelin and embelin-6g on XIAP expression, we investigated the effect of these drugs on the androgen receptor signaling axis which is a key regulatory gene involved in prostate cancer. First, we examined the 

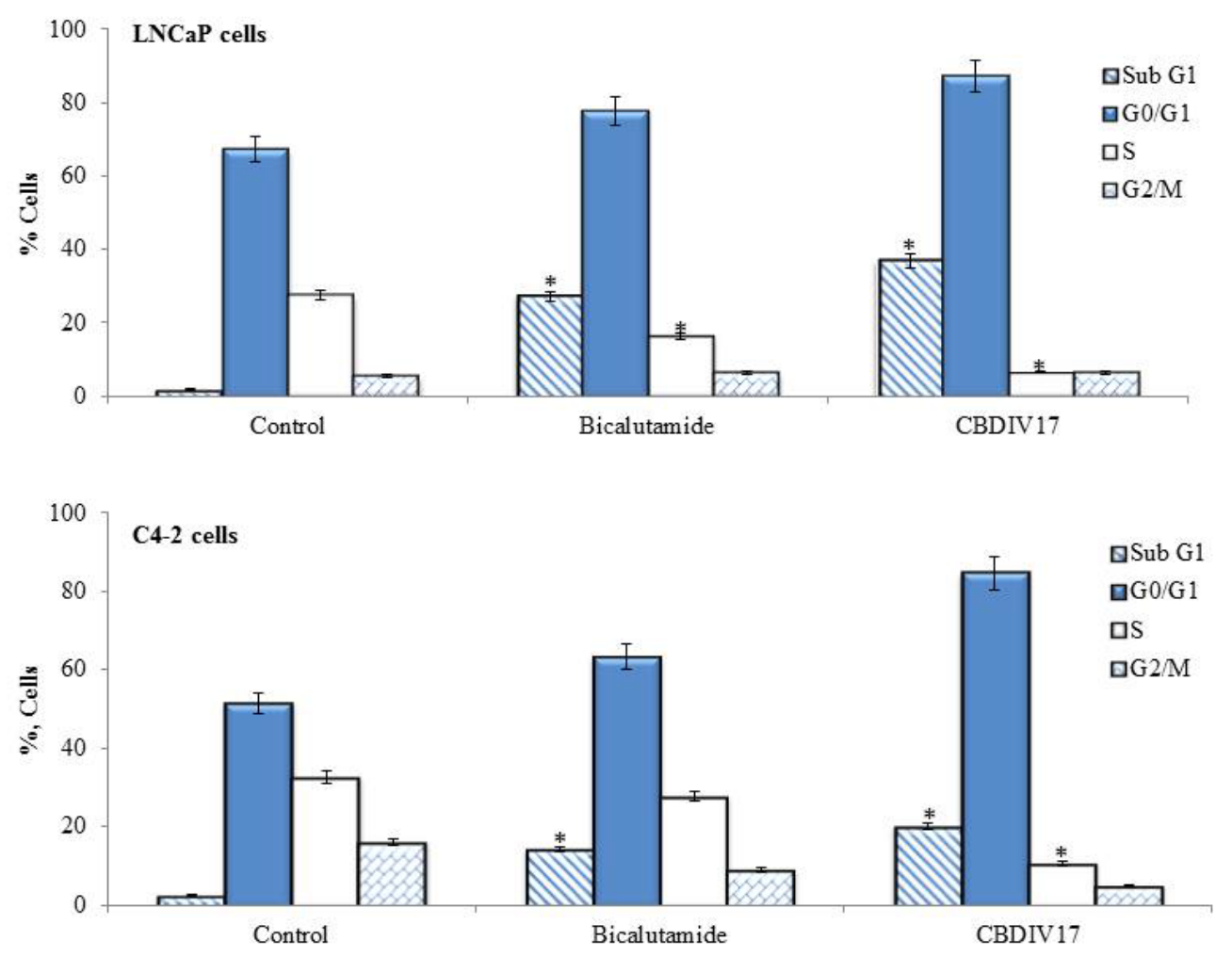

Figure 5-4. Effect of bicalutamide and CBDIV17 on apoptosis and cell cycle of LNCaP and C4-2 cells.

Cells were treated with antiandrogen $(25 \mu \mathrm{M})$ for $72 \mathrm{~h}$, stained with propidium iodide and analyzed on a flow cytometer. ${ }^{*} \mathrm{p}<0.05$ using student's unpaired t-test. 


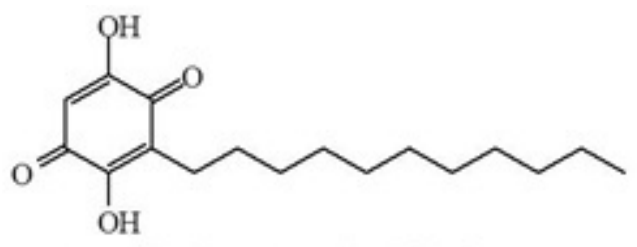

2,5-Dihydroxy-3-undecyl-[1,4] benzoquinone(Embelin)

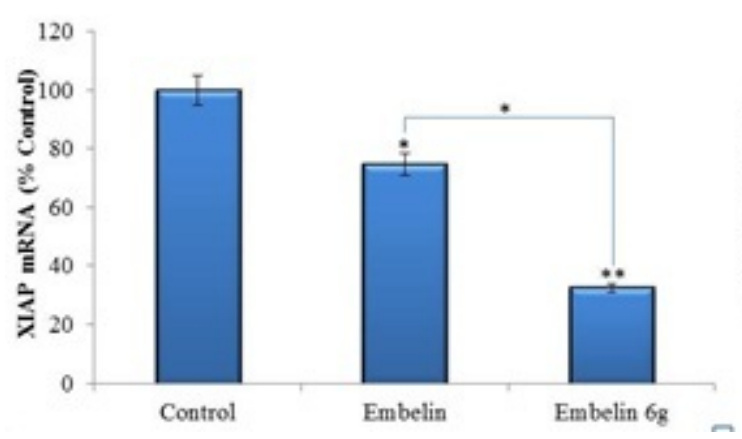

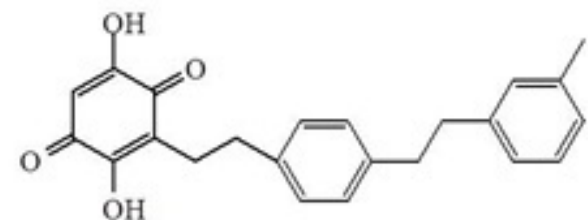

2,5-Dihydroxy-3-\{2-[4-(2-m-tolyl-ethyl)phenyl]-ethyl $\}-[1,4]$ benzoquinone (Embelin-6g)

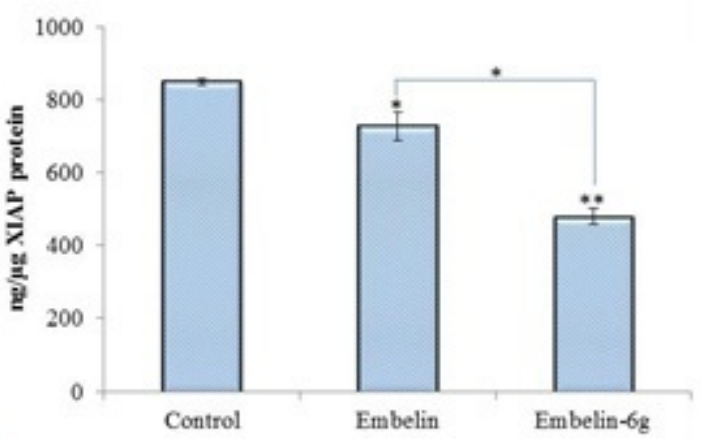

Figure 5-5. Embelin and embelin-6g suppress XIAP expression.

(A) Chemical structures of embelin and embelin-6g. (B) $1 \times 10^{5} \mathrm{C} 4-2$ cells were treated with embelin or embelin-6g for $48 \mathrm{~h}$, RNA extracted and XIAP expression at mRNA level determined using real-time RT-PCR. (C) $1 \times 10^{6} \mathrm{C} 4-2$ cells were treated with embelin or embelin-6g for $96 \mathrm{~h}$, protein isolated and XIAP expression at protein level determined using ELISA. ${ }^{*} \mathrm{p}<0.05,{ }^{* *} \mathrm{p}<0.01$ using student's unpaired t-test. 
effect of embelin and embelin-6g on AR expression. 1 x 105 C4-2 cells were treated with drug $(5 \mu \mathrm{M})$ for $48 \mathrm{~h}$ and the amount of AR message was quantitatively determined by real-time RT-PCR. There was a significant decrease in AR mRNA levels (Figure 5-6A) with both embelin and embelin-6g reducing AR levels by more than 50\% compared to control. Additionally, Western blot analysis revealed a decrease in AR protein amounts when C4-2 cells were treated with $5 \mu \mathrm{M}$ embelin or embelin-6g for $96 \mathrm{~h}$ (Figure 5-6B).

Furthermore, we evaluated AR transcriptional activity by examining mRNA expression of prostate specific antigen (PSA). We observed 50\% decrease in PSA mRNA levels for both drugs (Figure 5-6A). Additionally, we determined the effect of embelin and embelin-6g on intracellular PSA levels. Drug treatment resulted in a decrease in PSA protein expression compared to the control (Figure 5-6B). These results demonstrate the ability of embelin to modulate AR levels and show direct correlation between AR protein amount and its subsequent ability to transactivate PSA.

Embelin blocks DHT-stimulated AR nuclear translocation and phosphorylation

To determine whether embelin inhibits AR translocation in C4-2 cells we used confocal microscopy to determine the localization of AR in the nucleus. AR was diffusively distributed in the nucleus (Figure 5-6C) but cytoplasmic AR translocation to the nucleus was significantly decreased upon incubation with embelin. In contrast, treatment with $1 \mathrm{nM}$ DHT resulted in a majority of cytoplasmic AR translocating into the nucleus. Furthermore, since androgens stimulate phosphorylation of AR Ser81 especially in cells possessing T877A mutation, we examined the effect of embelin on AR phosphorylation [251]. C4-2 cells were treated with various concentrations of embelin in the presence or absence of DHT. A concentration-dependent effect of AR Ser81 phosphorylation post embelin treatment was observed strongly suggesting embelin suppressed DHT-induced AR phosphorylation (Figure 5-6D).

Embelin and its derivative inhibit prostate cancer cell growth

We next investigated the anticancer activity of embelin and embelin- $6 \mathrm{~g}$ by determining their $\mathrm{IC}_{50}$ values in $\mathrm{LNCaP}$ and $\mathrm{C} 4-2$ cells. Both drugs were found to be equally potent in inhibiting the proliferation of prostate cancer cells regardless of androgen status (Figure 5-7A). $\mathrm{IC}_{50}$ was approximately $6.5 \mu \mathrm{M}$.

To provide mechanistic insight into the effect of embelin and embelin-6g on cell proliferation, we examined whether these drugs modulate the expression of cyclin D1 which is a known marker of cell proliferation. Treatment of C4-2 cells with $5 \mu \mathrm{M}$ of embelin and embelin-6g for $48 \mathrm{~h}$ resulted in suppression of cyclin D1 expression by $\sim 22 \%$ (Figure 5-7B). Western blot analysis also revealed inhibition of cyclin D1 when cells were treated with drug for $96 \mathrm{~h}$ (Figure 5-7C). 


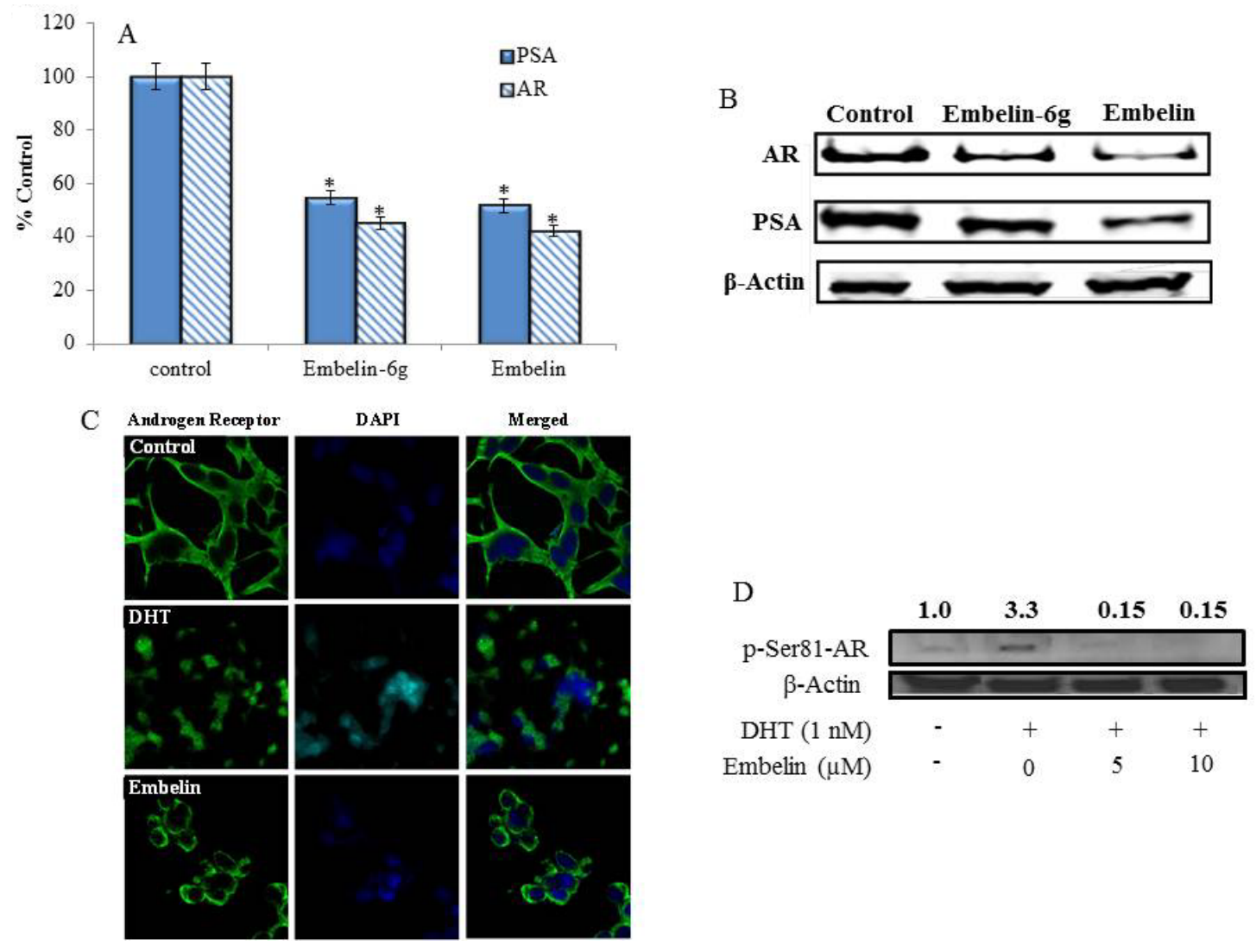

Figure 5-6. Effect of embelin and embelin-6g on AR and PSA expression.

(A) C4-2 cells were treated with embelin and embelin-6g (5 $\mu \mathrm{M})$ for $48 \mathrm{~h}$, RNA extracted and AR and PSA expression at mRNA level determined using real-time RT-PCR. (B) Cells were treated with embelin and embelin-6g $(5 \mu \mathrm{M})$ for $96 \mathrm{~h}$, protein isolated and AR and PSA expression at protein level determined using Western blot. (C) C4-2 cells were grown on chamber slides treated in the presence or absence of $5 \mu \mathrm{M}$ embelin for $12 \mathrm{~h}$ prior to addition of $1 \mathrm{nM}$ DHT. Cells were fixed, permeabilized and AR immunostained with rabbit anti-AR followed by FITC-conjugated secondary antibody and nucleus stained with DAPI. (D) Phosphorylated Ser81 AR protein levels in C4-2 cells were determined by Western blot analysis. Cells were grown in CSS and treated with Embelin ( 5 or $10 \mu \mathrm{M}$ ), in the presence or absence of $1 \mathrm{nM} \mathrm{DHT}$ for $48 \mathrm{~h}$. 

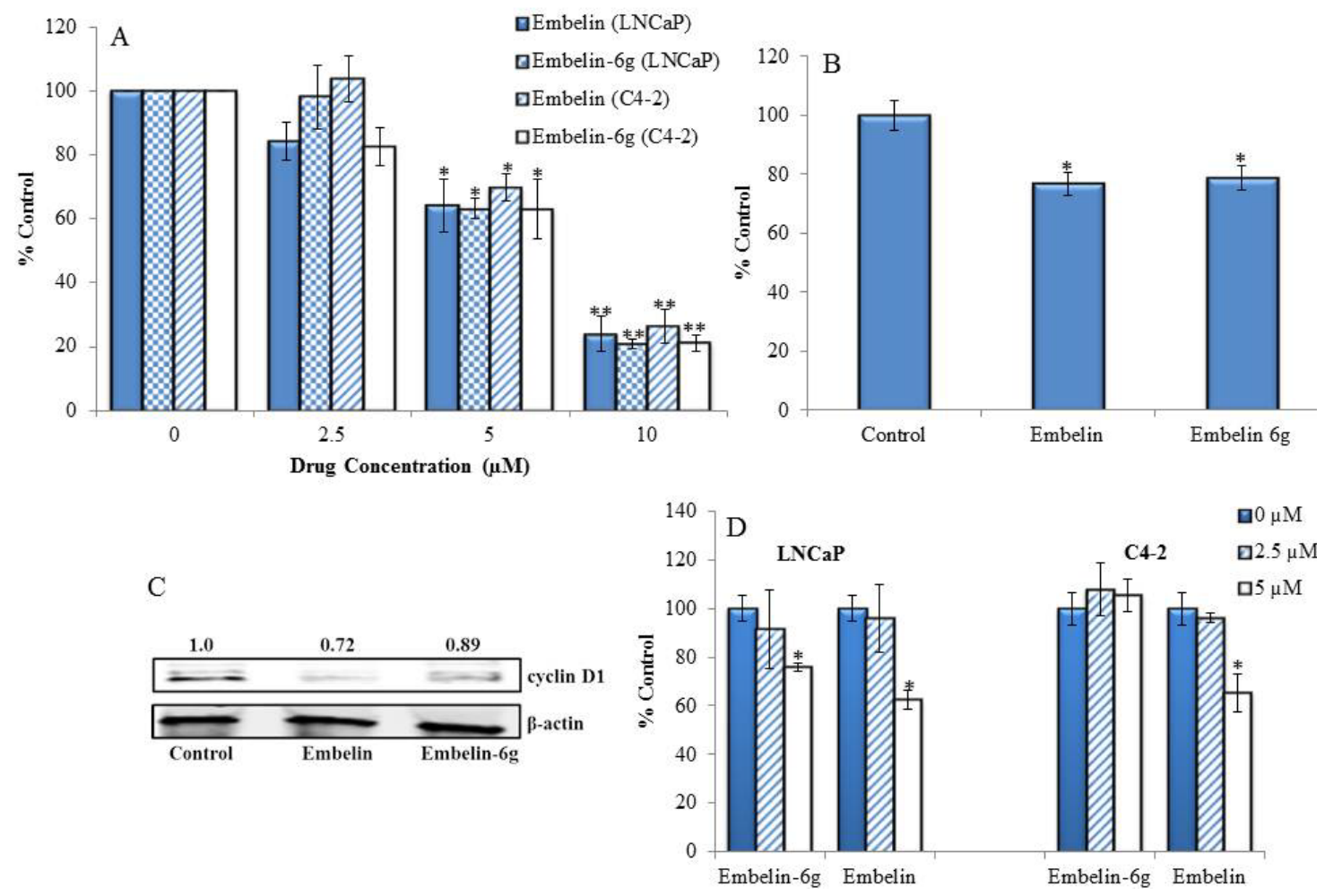

Figure 5-7. Effect of embelin and embelin-6g on prostate cancer cell proliferation and cyclin D1, expression.

(A) $\mathrm{LNCaP}$ and $\mathrm{C} 4-2$ cells were treated with XIAP inhibitor $(0-10 \mu \mathrm{M})$ for $96 \mathrm{~h}$ and cell viability measured using MTT assay. (B) Cells were treated with XIAP inhibitors $(5 \mu \mathrm{M})$ for $48 \mathrm{~h}$, total RNA extracted and cyclin D1 expression at mRNA level determined using real-time RT-PCR. (C) $1 \times 10^{6}$ cells were treated with XIAP inhibitors for $96 \mathrm{~h}$, protein isolated and cyclin D1 expression at protein level determined using Western blot. (D) $\mathrm{LNCaP}$ and C4-2 cells were treated with XIAP inhibitors $(0,2.5$ and $5 \mu \mathrm{M})$ for 24 and cell viability measured using MTT assay. ${ }^{*} \mathrm{p}<0.05,{ }^{* *} \mathrm{p}<0.01$ using student's unpaired t-test. 
Since we were interested in using a XIAP inhibitor which has fast acting activity for our combination study, we examined the anticancer activity of embelin and embelin$6 \mathrm{~g}$ in $\mathrm{LNCaP}$ and $\mathrm{C} 4-2$ cells at $18 \mathrm{~h}$ post treatment. Our results (Figure 5-7D) suggest embelin to be faster acting and more potent than embelin-6g in inhibiting cell growth especially in C4-2 cells. Therefore, we selected embelin as the XIAP inhibitor for further studies in our combination therapy.

Formulation of CBDIV17 and embelin-loaded PEG-b-p(CB-co-LA) micelles and their effect on prostate cancer cell growth and migration

Since CBDIV17 and embelin are extremely hydrophobic and we plan to test our combination therapy in vivo, we first formulated these drugs into Poly (ethylene glycol)b- poly(carbonate-co-lactide) (PEG-b-p(CB-co-LA)) micelles. Micelle size ranged from 83 to $90 \mathrm{~nm}$ and the presence of drug did not significantly affect micelles size.

Furthermore, (PEG-b-p(CB-co-LA)) micelles were monodisperse and had a PDI of approximately 0.11 . Encapsulation efficiency for CBDIV17 was $91.2 \pm 2.3 \%$ at $5 \%$ theoretical loading but dropped to $55.7 \pm 1.4 \%$ when theoretical loading increased to $10 \%$. In contrast, encapsulation efficiency for embelin was $38.4 \pm 1.0 \%$ at $5 \%$ theoretical loading and $35.0 \pm 0.9 \%$ at $10 \%$ theoretical loading. Therefore, a $5 \%$ theoretical loading was used for formulating micelles for further studies.

Following micelle formulation and characterization, we investigated whether the combination of CBDIV17 and embelin was more potent than their monotherapy in treating prostate cancer. C4-2 cells were treated with embelin $(5 \mu \mathrm{M})$ or CBDIV17 (25 $\mu \mathrm{M}$ ) alone or in combination for $24 \mathrm{~h}$. Combination therapy was found to be more effective than control or monotherapy (Figure 5-8A). Both embelin and CBDIV17 resulted in approximately $20 \%$ decrease in cell growth while combination therapy inhibited cell growth by about $70 \%$.

We also determined the effect of combining CBDIV17 and embelin on the migration potential of C4-2 cells using the scratch wound assay. We observed embelin to have no obvious effect on cell migration under our conditions whereas CBDIV17 had some modest effect. The group treated with the combination of CBDIV17 and embelin resulted in superior inhibition of cell migration compared to untreated control and each drug alone (Figure 5-8B).

Effect of CBDIV17 and embelin combination on apoptosis

Since CBDIV17 and embelin combination effectively inhibited cell proliferation in vitro, we further tested their effect on cell cycle and apoptosis in C4-2 cells. From Figure 5-9A, there was a decrease in percentage of cells in the $\mathrm{G} 2 / \mathrm{M}$ and $\mathrm{S}$ phases following treatment with CBDIV17. Treatment with 25 and $50 \mu \mathrm{M}$ decreased percent of cells in the $\mathrm{G} 2 / \mathrm{M}$ phase by $50 \%$ while percentage of cells in the $\mathrm{S}$ phase decreased by $30 \%$. Also, we found CBDIV17 showed dose-dependent increase in apoptosis (sub-G1). 

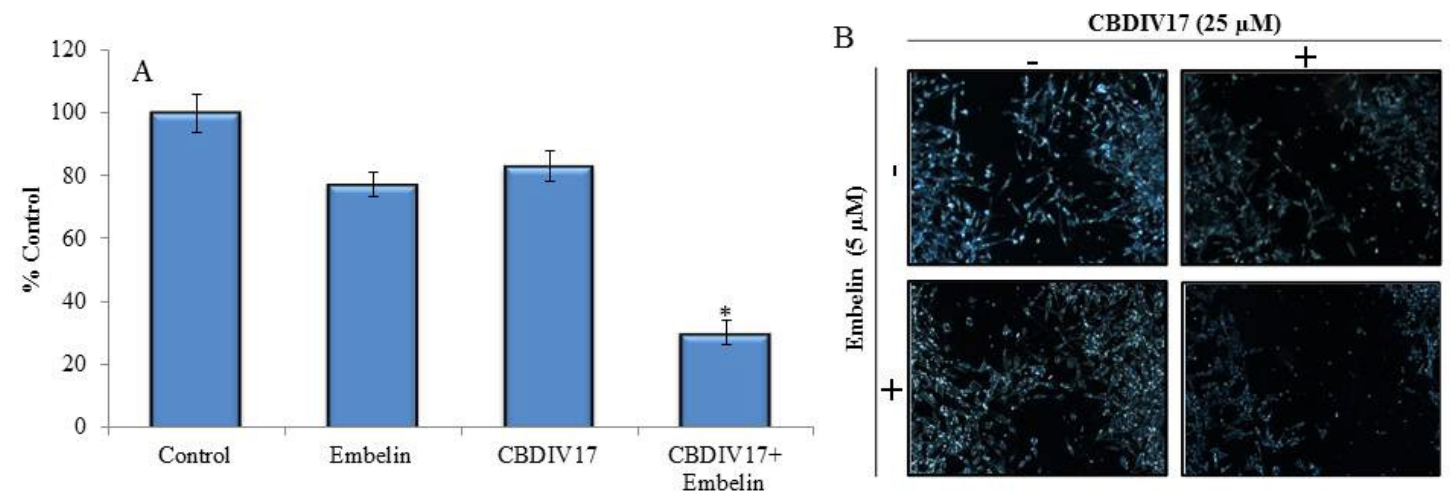

Figure 5-8. Effect of CBDIV17 and embelin combination on prostate cancer cell growth and migration.

(A) Anticancer effect of CBDIV17 and embelin, alone or in combination on C4-2 cells was determined following treatment of cells with $25 \mu \mathrm{M}$ of CBDIV17 and $5 \mu \mathrm{M}$ embelin for $24 \mathrm{~h}$. and cell viability determined by MTT assay. $* \mathrm{p}<0.05$ using student's unpaired t-test. (B) C4-2 cells were grown to 70\% confluence in six well plates and three parallel wounds made using pipette tip. Cells were treated with CBDIV17 $(25 \mu \mathrm{M})$ or embelin $(5$ $\mu \mathrm{M}$ ) alone or in combination for $48 \mathrm{~h}$ after which cells were washed with ice cold 1x PBS and imaged under microscope. 


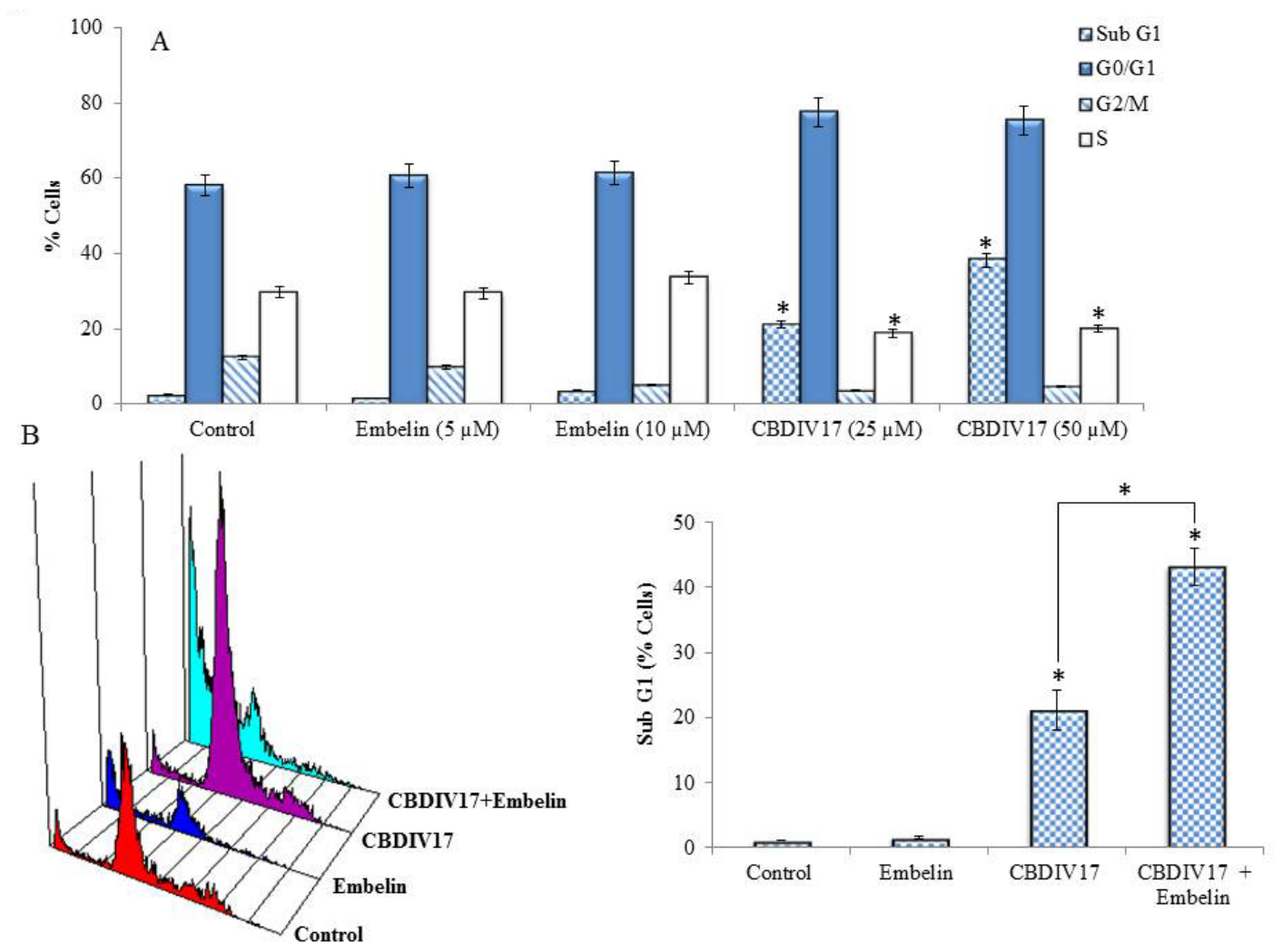

Figure 5-9. Effect of embelin and CBDIV17 combination on cell cycle and apoptosis.

(A) C4-2 cells were treated with vehicle, embelin (5 and $10 \mu \mathrm{M})$ and CBDIV17 (25 and $50 \mu \mathrm{M}$ ) for $72 \mathrm{~h}$, stained with propidium iodide and analyzed on a flow cytometer. (B) Cells were treated with vehicle, embelin $(5 \mu \mathrm{M})$ and CBDIV17 $(25 \mu \mathrm{M})$ alone or in combination for $72 \mathrm{~h}$, stained with propidium iodide and analyzed on a flow cytometer. Left panel, cell cycle distribution; Right panel, quantitative analysis of sub G1phase. * p $<0.05$ using student's unpaired t-test. 
The percentage of cells in the sub-G1 phase was around $21 \%$ at $25 \mu \mathrm{M}$ and $38 \%$ for 50 $\mu \mathrm{M}$. In contrast, percentage of cells in the sub-G1 phase did not differ significantly fromthat of control for the concentrations of embelin tested. Notably, we observed combination of CBDIV17 $(25 \mu \mathrm{M})$ and embelin $(5 \mu \mathrm{M})$ induced apoptosis more potently compared to control or either treatment alone. Specifically, percentage of cells in the subG1 phase for combination therapy was 40 -fold, 28 -fold and 2-fold more, compared to control, embelin and CBDIV17, respectively. Additionally, combination of CBDIV17 $(25 \mu \mathrm{M})$ and embelin $(5 \mu \mathrm{M})$ resulted in percentage of cells in the sub-G1 phase comparable to that of $50 \mu \mathrm{M}$ CBDIV17.

In vivo efficacy of CBDIV17 and embelin-loaded micelles in mouse xenografts

Since CBDIV17 and embelin combination therapy effectively inhibited prostate cancer growth in vitro, we determined the effect of this combination approach in C4-2 xenograft tumor model. CBDIV17 and embelin were formulated using PEG-b-p(CB-coLA) micelles and intratumoral injection of $10 \mathrm{mg} / \mathrm{kg}$ CBDIV17 and $10 \mathrm{mg} / \mathrm{kg}$ embelinloaded micelles was administered on days 0,3 and 7. CBDIV17 and embelin combination inhibited tumor growth more potently compared to the mice treated with empty micelles or monotherapy (Figure 5-10A). On day 7 (last day of treatment), the tumor size of the combination group was $50 \%$ of that in CBDIV17 group, $25 \%$ of that in embelin group and $18 \%$ of that in the control group (Figure 5-10B). Furthermore, the body weight loss of mice was less than $5 \%$ suggesting minimal toxicity of the treatment (Figure 5-10C). Additionally, combination therapy increased tumor-doubling time compared to monotherapy and control. For instance, the time it took for tumor to double from $50 \mathrm{~mm}^{3}$ to $100 \mathrm{~mm}^{3}$ are as follows: the combination group took 14 days; CBDIV17 group took 7 days and less than 3 days for embelin and control group. Taken together, these results demonstrate that CBDIV17 and embelin combination therapy effectively inhibited tumor growth and can prolong survival.

\section{Discussion}

Since prostate cancer is an androgen-dependent malignancy, current therapies including non-steroidal antiandrogens target the AR. However, first generation nonsteroidal anti-androgens do not completely hinder AR activity and eventually become agonists in tumor cells partly due to elevated levels of AR. Furthermore, progression to hormone refractory prostate cancer is also characterized by resistance to apoptosis. Particularly, XIAP is overexpressed and inhibits caspases 9 and 3 thus contributing to a defect in the apoptotic machinery [185-188]. Together, AR and XIAP overexpression may be a molecular reason leading to drug resistance and indicate that more potent antiandrogens, AR downregulating agents and XIAP inhibitors or their combination can effectively treat advanced prostate cancer. We have previously demonstrated that combination of bicalutamide and embelin is more effective in treating prostate cancer compared to monotherapy [11]. However, bicalutamide has limited efficacy in advanced prostate cancer (e.g., C4-2 cells) and assumes agonistic properties following prolonged 

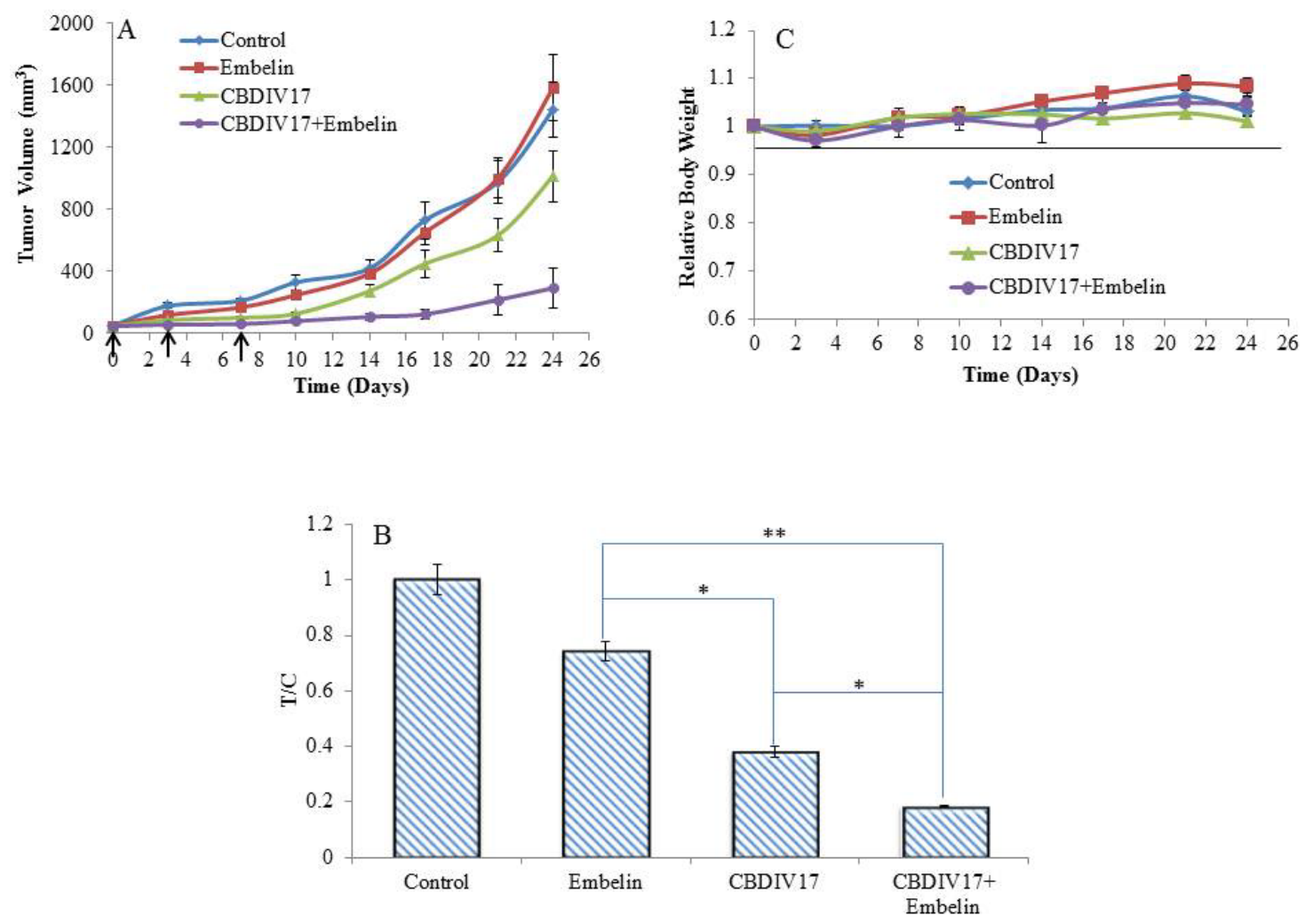

Figure 5-10. Effect of CBDIV17 and embelin-loaded micelles on growth of tumors derived from C4-2 prostate cancer cells in nude mice.

(A) Nude mice bearing $50 \mathrm{~mm}^{3} \mathrm{C} 4-2$ tumors were given an intratumoral injection of 10 $\mathrm{mg} / \mathrm{kg}$ CBDIV17 and $10 \mathrm{mg} / \mathrm{kg}$ embelin, alone or in combination (arrows indicate injection) and tumor size measured. Tumor growth regression was significantly higher for combination therapy group compared to control or monotherapy. Points are mean tumor size $(\mathrm{n}=7)$; bars, SE. (B) Tumor suppression $(\mathrm{T} / \mathrm{C})$ was computed as the ratio of mean tumor volume in treated groups compared with control when last injection was administered. (C) Relative body weight was expressed as the body weights at various times during treatment normalized with body weights at day 0 . ${ }^{*} \mathrm{p}<0.05,{ }^{* *} \mathrm{p}<0.01$ using student's unpaired t-test. 
treatment. Therefore, the primary objective of this study was to investigate whether a novel antiandrogen (CBDIV17) was more potent than bicalutamide and can effectively inhibit advanced prostate tumor growth when combined with XIAP inhibitor. We also further explored the molecular mechanism for our selected XIAP inhibitors.

To determine the comparative therapeutic benefits of CBDIV17 over bicalutamide, we evaluated the biological effects of both drugs in LNCaP and C4-2 cells by observing their effect on cell proliferation in the presence or absence of synthetic androgen and in inducing apoptosis. Our results show, CBDIV17 to be more potent than bicalutamide in inhibiting cell proliferation in a dose and time dependent manner (Figure 5-2). Furthermore, inhibition of cell growth was only modestly reversed in the presence of DHT. This may possibly be due to better cytotoxic effects or its superior ability at the molecular level to retain antagonism and hence bypass antiandrogen resistance. Our observation suggests CBDIV17 analog to be more potent than bicalutamide regardless of AR sensitivity or presence of synthetic androgen and therefore has the potential for treating advanced prostate cancer.

Chen et al. synthesized new XIAP inhibitors with better binding affinities than embelin by modifying its hydrophobic tail. It was shown that embelin- $6 \mathrm{~g}$ has a two-fold better binding affinity to XIAP BIR3 compared to embelin and was potent in inhibiting proliferation of human breast and prostate cancer cell lines [251]. Therefore, we were interested in how better XIAP binding affinity would translate in terms of potency in inhibiting cell growth and inducing apoptosis in prostate cancer cells. Although Chen et al. showed better binding affinity for embelin-6g they did not show its effect on XIAP gene expression. In our studies, we found embelin-6g to be more potent than embelin in repressing XIAP gene expression. At both the mRNA and protein levels, embelin-6g appeared to be twice as effective as embelin (Figure 5-5B and $\mathbf{C}$ ). This result may logically extend from embelin-6g's two-fold better binding affinity compared to embelin. However, we observed embelin and embelin-6g to exhibit similar ability in inhibiting prostate cancer cell growth despite the difference in their effect on XIAP expression. In fact, our findings suggest that embelin elicits a quicker therapeutic response compared to embelin-6g and was consequently more potent at short time intervals. Therefore, embelin was selected for combination studies with CBDIV17.

We also made an interesting finding regarding the ability of embelin and embelin$6 \mathrm{~g}$ to disrupt AR signaling in prostate cancer cells. These XIAP inhibitors also appear to decrease AR levels in LNCaP and C4-2 cells. Similar results have also been reported in the literature where other drugs exhibit the ability to decrease AR expression [252-254]. Examples of these AR down-regulating agents (ARDA) include quercetin, epicatechin, and curcumin. Purushottamachar et al. provided clear evidence concerning structural requirements common to ARDAs [255]. Typical features include one hydrophobic group, one aromatic group, and two hydrogen bond acceptors [255]. Both embelin and embelin$6 \mathrm{~g}$ satisfy these structural criteria and hence it may not be surprising that they are capable of depleting AR expression. 
Although the precise mode of action of embelin and embelin-6g in downregulating AR inhibition is not fully understood, it is likely that both drugs inhibit AR expression through a number of different mechanisms. Since both drugs decrease AR expression by more than $50 \%$ on the mRNA level, it is possible that mRNA stability or inhibition of AR mRNA synthesis may contribute to the effect of these drugs on AR expression levels. It is also possible that embelin and embelin-6g hinder androgen-AR binding or may disrupt the Hsp90-AR complex. The likelihood of these mechanisms which have also been reported by Liu et al. [252] would be consistent with our results where we showed the ability of embelin to prevent AR nuclear translocation from the cytoplasm to the nucleus in C4-2 cells (Figure 5-6C).

Combination therapy involving XIAP modulation is a promising approach for treating solid tumors including prostate cancer [11, 256, 257]. We observed CBDIV17 and embelin combination to be more potent in inhibiting prostate cancer cell growth and in inducing apoptosis in advanced prostate cancer compared to control or monotherapy. This superior effect was found to be supra-additive and is consistent with our previous findings which showed synergism even for simultaneous administration of bicalutamide and embelin in C4-2 cells [11]. The greater potency of CBDIV17 and embelin combination therapy may be attributed to the ability of embelin to lower the threshold required by XIAP to inhibit apoptosis thereby enhancing CBDIV17 activity several fold. From our findings, it is also possible that the ability of embelin to downregulate AR may play some role in the observed therapeutic effect of our combination therapy.

Since in vivo substantiation of our cellular observations is imperative for clinical translation, CBDIV17 and embelin were formulated into PEG-b-p(CB-co-LA) micelles to test our combination approach in vivo due to their extreme hydrophobicity and to avoid the detrimental side effects of solubilizing agents such as DMSO and Cremophor ${ }^{\circledR}$ EL [7]. CBDIV17 had higher drug loading compared to embelin. This may be due to the close structural similarity between CBDIV17 and bicalutamide for which PEG-b-p(CBco-LA) was specifally designed to enhance drug loading [170]. Consistent with our in vitro data, we found co-administration of embelin and CBDIV17 to be more potent in regressing prostate tumor growth compared to control or monotherapy (Figure 5-10A and B). Tumor volume at the last injection and at the end of experiment for the combination group was approximately $20 \%$ of control. A result demonstrating the ability of our combination therapy to suppress growth of advanced prostate cancer tumors. Also, CBDIV17 was effective in regressing tumor growth at the doses studied compared to embelin or control. However, tumor volume increased to $70 \%$ of control at the end of the experiment compared to $40 \%$ of control at the last injection. We found the embelin treated group to have tumor volume $75 \%$ of the control group when the last injection was administered. Nonetheless, there was no difference between embelin treated group and control at the end of the study. Our results are in agreement with the findings of Dai et al. [258]. However, they orally administered a 6-fold higher dose of embelin $(60 \mathrm{mg} / \mathrm{kg})$ twice a week for three weeks and used PC-3 xenograft tumors which are AR negative.

In conclusion, we have demonstrated that CBDIV17 is more potent than bicalutamide in regressing prostate tumor growth and inducing apoptosis regardless of 
AR status. Additionally, we have shown that the combination of CBDIV17 and embelin is more potent in inhibiting cell growth and inducing apoptosis compared to control or monotherapy in vitro and in vivo. Our findings also reveal a novel mechanism regarding the ability of embelin and embelin- $6 \mathrm{~g}$ to alter AR signaling pathway. We are currently investigating the mode of action by which embelin down-regulates AR gene expression. We believe such mechanistic insight will lead to the design of highly potent analogs. The current study provides evidence that CBDIV17 and embelin combination is a promising therapeutic approach for treating hormone refractory prostate cancer. 


\section{CHAPTER 6. LACTIC ACID AND CARBONATE BASED CROSSLINKED MICELLES FOR DRUG DELIVERY*}

\section{Introduction}

Polymeric micelles are presently under intense investigation as nano-vehicles for delivery of low molecular weight chemotherapeutic drugs. Key advantages of polymeric micelles include efficient solubilization of hydrophobic agents in their core [259-261] and the ability to be made site-specific. Furthermore, they are nanosized supramolecules which self-assemble into core-corona structures from amphiphilic block copolymers once above the critical micelle concentration (CMC) [261]. These intrinsic physicochemical properties of micelles often leads to prolonged blood circulation kinetics[262] and preferential tumor accumulation via the enhanced permeation and retention (EPR) effect $[13,46,195]$. Nonetheless, poor in vivo stability resulting in premature dissociation and subsequent untimely drug release has limited their clinical application [263-266]. Polymeric micelles become unstable either due to destabilization by plasma proteins (kinetic instability) or dilutions below their critical micelle concentration (CMC) (thermodynamic instability) upon systemic administration.

To facilitate clinical translation of polymeric micelle based therapeutics, recent research effort has focused on increasing micelle thermodynamic and kinetic stability using physical or chemical strategies. Since micelles with low CMC values have better thermodynamic stability, typical physical approaches involve strategically altering properties of amphiphilic copolymers which can affect the CMC (e.g., chain length and chemical composition) [170, 267]. For example, tailoring the hydrophobic core to contain aromatic moieties has been shown to improve micelle stability through $\pi-\pi$ interactions as demonstrated by decreased CMC values [170]. In practice, physically stabilized micelles are limited by their inability to prevent micelle destabilization caused by blood components.

In contrast, introduction of chemical covalent crosslinks into the shell, core or core-corona interface of micelles is a potential approach to stabilize micelles against both plasma proteins and sub-critical micelle concentrations [267-270]. Over the past decade, shell-crosslinked micelles have been extensively studied [271]. It has been shown that shell-crosslinked micelles remain stable upon infinite dilution compared to precursor micelles. High core mobility, greater versatility in core composition and properties and the membrane-like characteristics of the cross-linked shell layer which can improve encapsulation are advantages associated with shell-crosslinked micelles. However, one longstanding issue with shell-crosslinked micelles is their low crosslinking efficiency due to their need to be prepared at high dilution to prevent intermicellar crosslinking.

\footnotetext{
* Danquah M., Fujiwara T., and Mahato R.I. (2012) Lactic acid and carbonate-based crosslinked polymeric micelles for drug delivery. J. Polymer Sci Part A: Polymer Chemistry (Accepted for Publication).
} 
Furthermore, shell crosslinking tends to suppress the mobility of the hydrophilic corona chains (e.g., PEG).

Core-crosslinked micelles exhibit better crosslinking efficiencies since they do not need to be prepared at high dilution to prevent intermicellar crosslinking. Several groups have introduced crosslinkable groups into the hydrophobic portion of the block copolymer which may be polymerized after micellation to lock the micelle structure. For instance, Kataoka's group reported core-crosslinked micelles prepared using poly (ethylene glycol)- $b$-polylactide (PEG- $b$-PLA) possessing methacryloyl groups at the end of the PLA chain [272, 273]. These methacryloyl groups could be photopolymerized using ultraviolet (UV) light or polymerized thermally via the addition of a radical initiator. In another paper, $\mathrm{Hu}$ et. al. covalently stabilized micelle architecture using a hydrophobic polymer block containing crosslinkable double bonds [274]. Recently, Garg and coworkers used click chemistry to introduce hydrolyzable cross-links in the core of poly-(ethylene oxide)-block-poly( $\varepsilon$-caprolactone) (PEO-b-PCL) micelles [275]. These crosslinked micelles also demonstrated enhanced stability under diluted conditions without any detrimental effect on the drug encapsulation and in vitro drug release profiles.

Armes and coworkers have elegantly stabilized micelles by crosslinking the corecorona interface using triblock copolymers in which the reactive groups capable of undergoing cross-linking are located in the central block [276]. Interfacial crosslinking is potentially more beneficial than shell or core-crosslinked micelles since it combines the advantages of both methods and may be prepared at high concentrations without intermicellar cross-linking and aggregation. In another study, $\mathrm{Xu}$ and coworkers showed reduction-sensitive reversibly interfacially crosslinked micelles to be stable against extensive dilution and physiological salt conditions and retained most drugs even at concentrations below the CMC [277]. Yang et al. recently reported preparing interface crosslinked micelles using PEG- $b$-poly(acryloyl carbonate)- $b$-polycaprolactone (PEG- $b$ PAC- $b$-PCL) triblock copolymer [278]. Once micelles were prepared, the double bonds in the acryloyl carbonate were photo-crosslinked using UV light and a photo initiator.

Our group has developed a series of thermodynamically stable (low CMCs) lactic acid- and carbonate-based copolymers for micellar drug delivery [170, 247]. To further enhance micelle stability and controlled drug release to make our delivery systems clinically relevant, we herein report on the synthesis, characterization and in vitro evaluation of methoxy poly (ethylene glycol)- $b$-poly(carbonate- $c o$-lactide-co-5-methyl-5allyloxycarbonyl-1,3-dioxan-2-one) [mPEG- $b$-P(CB-co-LA-co-MAC)] and methoxy poly (ethylene glycol)- $b$-poly (5-methyl-5-allyloxycarbonyl-1,3-dioxan-2-one)- $b$ poly(carbonate-co-lactide) [mPEG- $b$-PMAC- $b$-P(CB-co-LA)] biodegradable copolymers for core and core-corona interface crosslinked micelles. Effect of polymer composition and crosslinking moiety location on key micelle properties was also investigated. Selfaggregation behavior, morphology and stability of non-crosslinked and crosslinked micelles were determined by dynamic light scattering (DLS), transmission electron microscopy (TEM), ${ }^{1}$ H NMR spectroscopy and fluorescence spectroscopy. Furthermore, 
influence of crosslink location on micelle stability against physiological simulating dilution and serum conditions as well as drug release was investigated.

\section{Materials and Methods}

Materials

2,2-bis(hydroxymethyl) propionic acid, methoxy poly(ethylene glycol) (mPEG, $M \mathrm{n}=5000, \mathrm{PDI}=1.03)$, tin(II) 2-ethylhexanoate $\left(\mathrm{Sn}(\mathrm{Oct})_{2}\right)$, dimethylaminopyridine (DMAP), allyl bromide and benzyl bromide were purchased from Sigma Aldrich (St. Louis, MO) and used as received. L-lactide (LA) was purchased from PURAC Biochem bv (Gorinchem, The Netherlands) and recrystallized from toluene several times. 1-Ethyl3-(3-dimethylaminopropyl) carbodiimide hydrochloride (EDC) and hydroxybenzotriazole (HOBt) were obtained from AK Scientific (Palo Alto, CA). 1,8-Diazabicyclo[5.4.0] undec-7-ene (DBU) was purchased from Sigma Aldrich, dried over calcium hydride and distilled. All other reagents were obtained from Sigma Aldrich and used without further purification.

Synthesis of 5-Methyl-5-allyl-1,3-dioxane-2-one

\section{Method 1}

5-methyl-5-benzyloxycarbonyl-1,3-dioxane-2-one (CB) monomer was first synthesized as described by Danquah et al. [170]. Briefly, 2,2bis(hydroxymethyl)propionic acid $(0.168 \mathrm{~mol})$ and potassium hydroxide $(0.169 \mathrm{~mol})$ were dissolved in $125 \mathrm{~mL}$ of dimethylformamide (DMF) by heating at $100^{\circ} \mathrm{C}$ for $1 \mathrm{~h}$. Subsequently, benzyl bromide $(0.202 \mathrm{~mol})$ was added dropwise to the reaction mixture and allowed to stir at $100{ }^{\circ} \mathrm{C}$ for $15 \mathrm{~h}$. The solvent was then removed under reduced pressure, the residue dissolved in ethyl acetate $(150 \mathrm{~mL})$, hexanes $(150 \mathrm{~mL})$ and water $(100 \mathrm{~mL})$, and dried over $\mathrm{Na}_{2} \mathrm{SO}_{4}$. The organic layer solvent was removed under vacuum and recrystallized from toluene to yield pure benzyl 2,2-bis(methylol)propionate. Next, benzyl 2,2-bis(methylol)- propionate $(0.05 \mathrm{~mol})$ was dissolved in a mixture of $\mathrm{CH}_{2} \mathrm{Cl}_{2}$ $(150 \mathrm{~mL})$ and pyridine $(25 \mathrm{~mL})$ and the solution chilled to $-78^{\circ} \mathrm{C}$. A solution of triphosgene ( $25 \mathrm{mmol}$ ) in $\mathrm{CH}_{2} \mathrm{Cl}_{2}$ was added dropwise to the reaction mixture over $1 \mathrm{~h}$ and stirred for an additional $2 \mathrm{~h}$ at the room temperature. The reaction was then quenched with saturated aqueous $\mathrm{NH}_{4} \mathrm{Cl}(75 \mathrm{~mL})$ and the organic layer sequentially washed with 1 $\mathrm{M}$ aqueous $\mathrm{HCl}(3 \times 100 \mathrm{~mL})$ and saturated aqueous $\mathrm{NaHCO}_{3}(1 \times 100 \mathrm{~mL})$, dried with $\mathrm{Na}_{2} \mathrm{SO}_{4}$, and evaporated under vacuum to give 5-methyl-5-benzyloxycarbonyl-1,3dioxane-2-one (CB). The crude product was purified by recrystallization from ethyl acetate to obtain white crystals. 
5-Methyl-5-allyl-1,3-dioxane-2-one (MAC) monomer was then synthesized by first subjecting $\mathrm{CB}$ to catalytic hydrogenation and reacting the subsequent product with allyl alcohol. In brief, CB (3 g) was dissolved in ethyl acetate $(30 \mathrm{~mL})$ containing $600 \mathrm{mg}$ $\mathrm{Pd} / \mathrm{C}$. The Parr bottle was purged thrice with $\mathrm{H}_{2}$, charged to 50 psi and the reaction allowed to proceed for $3 \mathrm{~h}$. Subsequently, $\mathrm{Pd} / \mathrm{C}$ catalyst was separated by centrifugation, the solvent filtered and evaporated under vacuum to give 5-methyl-2-oxo-1,3-dioxane-5carboxylic acid (MTC-OH) as a white crystal. Next, MTC-OH (3 mmol), allyl alcohol (2.5 mmol), HOBT (3.6 mmol), and EDC (4.5 mmol) were dissolved in dimethylformamide (DMF) $(20 \mathrm{~mL})$ to which triethylamine (TEA) $(3.5 \mathrm{mmol})$ was later added and the reaction allowed to proceed for $18 \mathrm{~h}$. Afterward, $20 \mathrm{~mL}$ ethyl acetate was added to the mixture and then washed with water. The organic layer was dried over $\mathrm{Na}_{2} \mathrm{SO}_{4}$, evaporated under vacuum and the crude product purified by column chromatography to give MAC as white crystals.

\section{Method 2}

MAC was synthesized as described by $\mathrm{Hu}$ et al.[274]. Briefly, a mixture of 2,2bis(hydroxymethyl)propionic acid $(9.0 \mathrm{~g}, 67.11 \mathrm{mmol})$, potassium hydroxide ( $88 \%$ assay; $4.30 \mathrm{~g}, 76.79 \mathrm{mmol})$, and DMF $(50 \mathrm{~mL})$ was heated to $100^{\circ} \mathrm{C}$ for $1 \mathrm{~h}$ with stirring at which point a homogenous potassium salt solution was formed. Allyl bromide $(5.8 \mathrm{~mL}$, $67.11 \mathrm{mmol}$ ) was added dropwise to the warm solution, and stirring was continued at $45^{\circ} \mathrm{C}$ for $48 \mathrm{~h}$. Upon completion of the reaction, the mixture was cooled, solvent removed under vacuum and the residue dissolved in methylene chloride $(200 \mathrm{~mL})$ and water $(100$ $\mathrm{mL})$. The organic layer was retained, washed with water $(100 \mathrm{~mL})$, dried $\left(\mathrm{Na}_{2} \mathrm{SO}_{4}\right)$, and evaporated to yield a viscous yellowish liquid (70\%).

Allyl 2,2-bis(methylol)propionate (10 g, $58 \mathrm{mmol})$ and ethyl chloroformate (16.5 $\mathrm{mL}, 0.173 \mathrm{~mol})$ were dissolved in tetrahydrofuran (THF) $(200 \mathrm{~mL})$, and the solution was stirred at $0^{\circ} \mathrm{C}$ for 30 min under $\mathrm{N}_{2}$. Subsequently, TEA $(24.2 \mathrm{~mL}, 0.173 \mathrm{~mol})$ was added dropwise over $30 \mathrm{~min}$, after which the reaction mixture was removed from the ice bath and stirred at room temperature overnight. TEA- $\mathrm{HCl}$ precipitate was filtered and the filtrate concentrated under reduced pressure. The ensuing solid was recrystallized from THF/ether to obtain white crystals $(8 \mathrm{~g})$.

\section{Synthesis of mPEG-b-P(lactide-co-carbonate-co-MAC)}

The following reaction mixture was prepared in a fume hood under ambient atmosphere. mPEG (150 mg), lactide (100 mg), CB (100 mg) and MAC (20 mg) were dissolved in $\mathrm{CH}_{2} \mathrm{Cl}_{2}(6 \mathrm{~mL})$ in a $25 \mathrm{~mL}$ reaction vessel. DBU $(30 \mu \mathrm{L})$ was added and the reaction carried out for $3 \mathrm{~h}$ after which benzoic acid $(40 \mathrm{mg})$ was added. The resulting solution was concentrated to approximately $50 \%$ of the initial volume and added dropwise into excess of cold isopropanol with stirring. The polymer was then dried under vacuum. 
Synthesis of mPEG-b-PMAC-b-P(lactide-co-carbonate)

The following reaction mixture was prepared in a fume hood under ambient atmosphere. mPEG (150 mg) and MAC (40 mg) were dissolved in $\mathrm{CH}_{2} \mathrm{Cl}_{2}(3 \mathrm{~mL})$ in a 25 $\mathrm{mL}$ reaction vessel. DBU $(15 \mu \mathrm{L})$ was added and the reaction carried out for $2 \mathrm{~h}$ to yield mPEG- $b$-PMAC. To this solution a mixture $(3 \mathrm{~mL})$ of CB $(100 \mathrm{mg})$ and LA $(100 \mathrm{mg})$ was infused into the reaction vessel at a rate of $1 \mathrm{~mL} / \mathrm{min}$. DBU $(15 \mu \mathrm{L})$ was then added and the reaction allowed to proceed for $3 \mathrm{~h}$, after which benzoic acid (40 mg) was added. PEG- $b$-PMAC- $b$-P(CB-co-LA) copolymer was purified by concentrating the reaction mixture to approximately $50 \%$ of the initial volume, added dropwise into excess of cold isopropanol and dried under vacuum.

Polymer characterization

Nuclear magnetic resonance (NMR)

${ }^{1} \mathrm{H}$ NMR, ${ }^{13} \mathrm{C}$ NMR, 2D-COSY and ${ }^{1} \mathrm{H}_{-}{ }^{13} \mathrm{C}$ HSQC spectra were recorded on a Bruker $\left(400 \mathrm{MHz}, \mathrm{T}=25^{\circ} \mathrm{C}\right)$ using deuterated chloroform $\left(\mathrm{CDCl}_{3}\right)$ and deuterated dimethyl sulfoxide (DMSO- $\mathrm{d}_{6}$ ) as solvents. The chemical shifts were calibrated using tetramethylsilane as an internal reference and given in parts per million.

\section{Gel permeation chromatography (GPC)}

A Waters GPC system equipped with a GPC column (AM Gel 103/5) and a differential refractive index detector was used to determine weight $\left(\mathrm{M}_{\mathrm{w}}\right)$ and number $\left(\mathrm{M}_{\mathrm{n}}\right)$ average molecular weight and polydispersity index (PDI) of synthesized copolymers. THF was used as an eluent at a flow rate of $1 \mathrm{~mL} / \mathrm{min}$. A series of narrow polystyrene standards $(700-40000 \mathrm{~g} / \mathrm{mol})$ were used for calibration and the data was processed using a Breeze 2 software.

Infrared (IR) spectra

Copolymer composition and degree of crosslinking was confirmed with Fourier Transform infrared (FT-IR) spectra using a Perkin-Elmer FT-IR spectrometer.

Critical micelle concentration (CMC)

Fluorescence spectroscopy was used to estimate the critical micelle concentration (CMC) of mPEG- $b$-P(LA- $c o-\mathrm{CB}-c o-\mathrm{MAC})$ and mPEG- $b$-PMAC- $b$-P(CB-co-LA) copolymers using pyrene as a hydrophobic fluorescent probe as described previously 
[11]. Fluorescence spectra of pyrene were recorded with a Molecular Devices SpectraMax M2/M2e spectrofluorometer (Sunnyvale, CA) with excitation wavelength $338 \mathrm{~nm}\left(\mathrm{I}_{3}\right)$ and $333 \mathrm{~nm}\left(\mathrm{I}_{1}\right)$ and emission wavelength of $390 \mathrm{~nm}$. The intensity ratio $\left(\mathrm{I}_{3} / \mathrm{I}_{1}\right)$ was plotted against the logarithm of polymer concentration and CMC obtained as the point of intersection of two tangents drawn to the curve at high and low concentrations, respectively.

Preparation of micelles and crosslinking

mPEG- $b$-P(LA-co-CB-co-MAC) and mPEG- $b$-PMAC- $b$-P(CB-co-LA) micelles were prepared using the film sonication method as previously described with slight modifications [11]. Briefly, $20 \mathrm{mg}$ of the copolymer was dissolved in $\mathrm{CH}_{2} \mathrm{Cl}_{2}(2 \mathrm{~mL})$. The mixture was sonicated for 5 min to ensure homogeneity and the solvent evaporated under a flow of $\mathrm{N}_{2}$. The resulting film was hydrated $(10 \mathrm{~mL})$ and sonicated for $10 \mathrm{~min}$ using a Misonix ultrasonic liquid processor (Farmingdale, NY) with an amplitude of 70. The ensuing formulation was then centrifuged at 5,000 rpm for $10 \mathrm{~min}$ and the supernatant filtered using a $0.22 \mu \mathrm{m}$ nylon filter.

Micelles were stabilized by polymerization of the allyl moieties. Briefly, the micelle solution was first deoxygenated by bubbling with argon for $1 \mathrm{~h}$. A solution of 2,2-azo-bis(isobutyronitrile) (AIBN) (1.0 wt. \% of polymer) in THF was then added and stirred for $2 \mathrm{~h}$ followed by heating to $70^{\circ} \mathrm{C}$ for $24 \mathrm{~h}$.

Drug loading and encapsulation efficiency

Bicalutamide loaded micelles were prepared as described above. $1 \mathrm{mg}$ drug and $19 \mathrm{mg}$ copolymer were dissolved in $\mathrm{CH}_{2} \mathrm{Cl}_{2}(2 \mathrm{~mL})$ and evaporated under a stream of $\mathrm{N}_{2}$. The film was then hydrated with $10 \mathrm{~mL}$ of PBS, sonicated and filtered through a $0.22 \mu \mathrm{m}$ filter. Acetonitrile was used to extract the loaded drug and the amount of bicalutamide determined using UV spectroscopy at $270 \mathrm{~nm}$. Drug loading content and encapsulation efficiency were then determined using Equations 6-1 and 6-2 as follows:

$$
\begin{gathered}
\text { drug loading density }=\frac{\text { weight of drug in micelle }}{\text { weight of micelle }} \times 100 \% \\
\text { drug encapsulation efficiency }=\frac{\text { weight of drug in micelle }}{\text { weight of drug originally fed }} \times 100 \%
\end{gathered}
$$

Particle size distribution and morphology

Mean particle size (intensity mean) and size distribution of non-crosslinked and crosslinked micelles were determined by dynamic light scattering (DLS) using a Zetasizer (Malvern Instruments, Worcestershire, UK) at a $1 \mathrm{mg} / \mathrm{mL}$ polymer 
concentration. Samples were analyzed at room temperature with a $90^{\circ}$ detection angle and the mean micelle size was obtained as a Z-average. Five repeat measurements were performed and data reported as the mean diameter $\pm \mathrm{SD}$. Non-crosslinked and crosslinked micelles prepared using mPEG- $b$-P(LA-co-CB-co-MAC) and mPEG- $b$ PMAC- $b$-P(CB-co-LA) copolymers were visualized using a JEM-100S (Japan) transmission electron microscope (TEM). Micelles were loaded on a copper grid, followed by blotting of excess liquid prior to negative staining with $1 \%$ uranyl acetate. The grid was visualized under the electron microscope at $60 \mathrm{kV}$ and magnifications ranging from $50,000 x$ to $100,000 x$.

In vitro drug release from micelles

The dialysis technique was used to study the release profile of bicalutamide from non-crosslinked and crosslinked micelles in phosphate buffered saline (PBS) (pH 7.2) with $0.1 \%$ Tween-80. Bicalutamide-loaded micelles with a final bialutamide concentration of $0.2 \mathrm{mg} / \mathrm{ml}$ were placed into a dialysis membrane with a molecular weight cut-off of $2000 \mathrm{Da}$ and dialyzed against $50 \mathrm{ml} \mathrm{PBS}(\mathrm{pH} \mathrm{7.2)}$ in a thermocontrolled shaker with a stirring speed of $150 \mathrm{rpm} .1 \mathrm{ml}$ samples were taken at specified times and assayed with a validated UV spectrophotometer by measuring the absorbance of the solution at $270 \mathrm{~nm}$. The cumulative amount of drug released into the media at each time point was evaluated as the percentage of total drug release to the initial amount of the drug. All experiments were performed in triplicate and the data reported as the mean of the three individual experiments.

\section{Assessment of in vitro micelle stability}

The stability of non-crosslinked and crosslinked micelles in PBS (pH 7.4) and under physiologically simulating conditions $(45 \mathrm{mg} / \mathrm{mL}$ BSA) was assessed by DLS. For stability in serum, micelle solution at a final polymer concentration of $20 \mu \mathrm{g} / \mathrm{mL}$ was incubated in BSA $(45 \mathrm{mg} / \mathrm{mL})$ at $37^{\circ} \mathrm{C}$ for 24 and $48 \mathrm{~h}$ with gentle shaking at $100 \mathrm{rpm}$. Particle size distribution of $1 \mathrm{~mL}$ aliquots was determined by DLS $(n=3)$.

In vitro cytotoxicity of bicalutamide-loaded micelles

The ability of bicalutamide-loaded PEG- $b$-PMAC- $b$-P(CB-co-LA) and PEG- $b$ $\mathrm{P}(\mathrm{CB}-\mathrm{co}$-LA-co-MAC) non-crosslinked $(\mathrm{NCM})$ and crosslinked micelles $(\mathrm{CM})$ to inhibit cell proliferation was evaluated using LNCaP human prostate cancer cell line. $3 \times 10^{3}$ cells were incubated with bicalutamide-loaded micelle formulations $(0,25$ and $50 \mu \mathrm{M}$ of drug) for 24 and $48 \mathrm{~h}$. At the end of treatment, MTT solution $(10 \% \mathrm{v} / \mathrm{v})$ was added to each well, incubated for $4 \mathrm{~h}$, residual formazan crystals solubilized with DMSO and the plate analyzed using a microplate reader $(560 \mathrm{~nm})$. Cell viability was expressed as a percentage of control and data reported as the mean of triplicate experiments. 


\section{Results}

Synthesis and characterization of 5-Methyl-5-allyl-1,3-dioxane-2-one

To facilitate micelle stabilization via crosslinking, we synthesized MAC which is a cyclic monomer containing a double bond functional moiety. Figure 6-1A illustrates the reaction pathway for synthesizing the MAC monomer. Drawing from our experience in synthesizing six-membered cyclic carbonate monomers, we first synthesized MAC using method 1 according to the procedure previously reported by Pratt et al.[231] with slight modification. This method allowed the synthesis of 5-methyl-5benzyloxycarbonyl-1,3-dioxane-2-one (CB) intermediate which is an integral component of our lactic-acid and carbonate-based copolymers. To this end, 2,2-

bis(hydroxymethyl)propionic acid was reacted with benzyl bromide to give benzyl 2,2bis(methylol)propionate which was then reacted with triphosgene to obtain CB (MW:250 $\mathrm{g} / \mathrm{mol}$ ). The chemical structure of $\mathrm{CB}$ was confirmed using mass and 1H NMR spectroscopy and our results matched the literature [170, 247]. CB was then dehydrogenated using $\mathrm{Pd} / \mathrm{C}$ catalyst to give 5-methyl-2-oxo-1,3-dioxane-5-carboxylic acid (MTC-OH; 30\% of CB) (MW:160 g/mol). Next, MTC-OH was coupled to allyl alcohol using EDC HOBt chemistry and purified using column chromatography to obtain MAC.

However, since we had large quantities of CB from previous studies, coupled with significant yield drops in step 1C (Figure 6-1A) and the time consuming column purification step associated with this method, we explored an alternative synthesis route (method 2). In this method, 2,2-bis(hydroxymethyl)propionic acid was reacted with allyl bromide to yield allyl 2,2-bis(methylol)propionate (MW: $174 \mathrm{~g} / \mathrm{mol}$ ) which was subsequently reacted with ethyl chloroformate and TEA to give MAC (70\%) and purified by recrystallization. Figure 6-1B shows the $1 \mathrm{H}$ NMR spectrum $(\mathrm{CDCl} 3)$ of MAC with peak assignments. The signal $\mathrm{d}$ at $5.8 \mathrm{ppm}$ and e at 5.23-5.42 ppm are characteristic of the acryloyl protons in the monomer; while the signal $\mathrm{b}$ at 4.2 and $4.7 \mathrm{ppm}$ denote methylene protons present in the carbonate ring. Other peak assignments include signals $\mathrm{a}$ and $\mathrm{c}$ at 1.38 and $4.72 \mathrm{ppm}$, assigned to methyl protons in the carbonate ring and methylene protons next to the ester, respectively. The structure of MAC was further confirmed using FT-IR spectrometry (Figure 6-2). Prominent absorbance peaks were observed at $1651 \mathrm{~cm}-1$ and $1731 \mathrm{~cm}-1$ for $\mathrm{C}=\mathrm{C}$ and $\mathrm{C}=\mathrm{O}$ stretches, respectively.

Synthesis and characterization of mPEG-b-PMAC-b-P(CB-co-LA) copolymer

mPEG- $b$-PMAC- $b$-P(CB-co-LA) copolymer was obtained by first reacting $\mathrm{mPEG}$ and MAC to give mPEG- $b$-PMAC copolymer followed by the addition of CB and LA (Figure 6-3). A kinetic study was performed to determine the optimal time required before the addition of $\mathrm{CB}$ and LA monomer. From Figure 6-4A and B, a maximum MAC conversion of $80 \%$ was achieved at a time of 105 min and maintained at 120 mins 


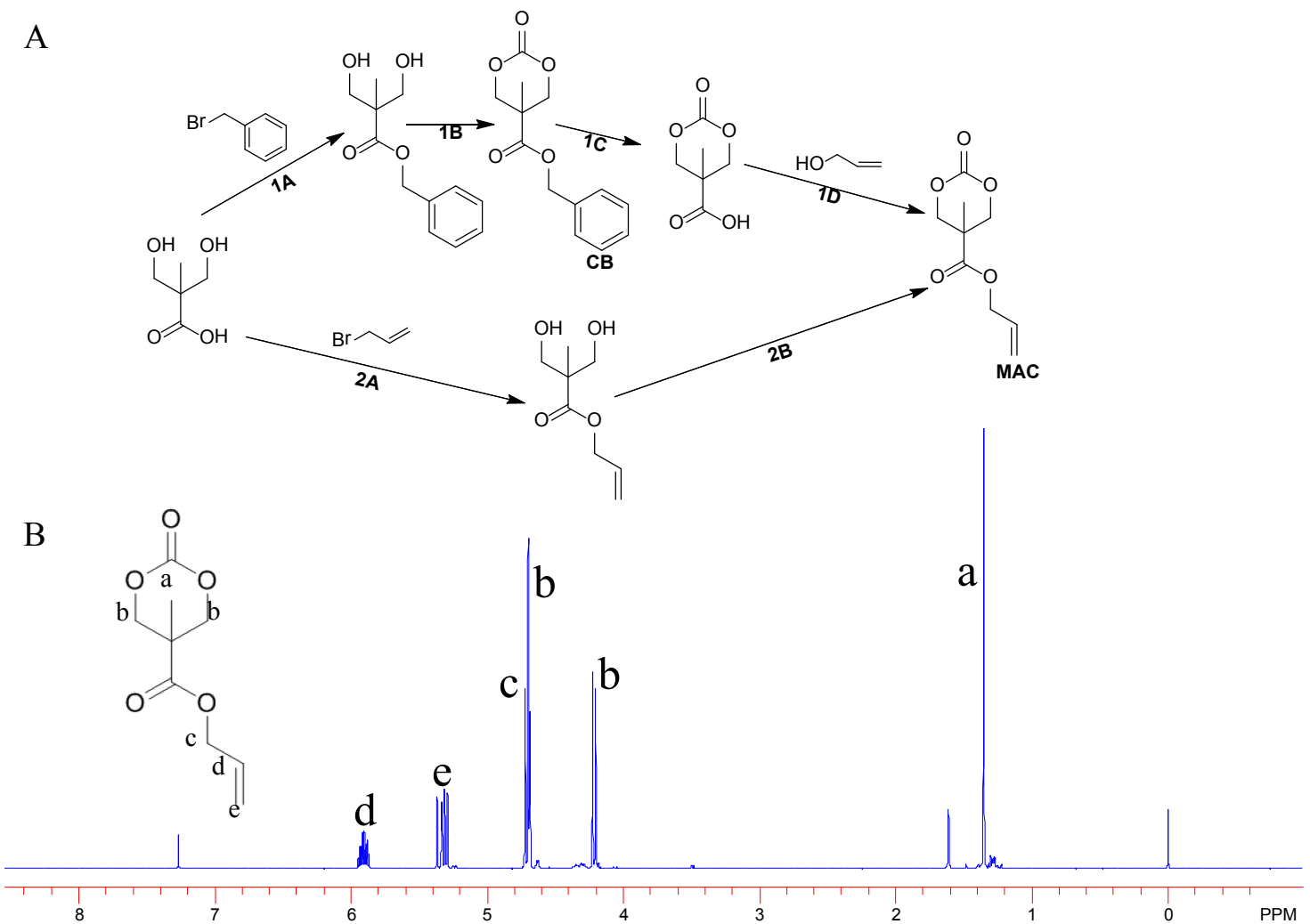

Figure 6-1. Synthesis and characterization of 5-methyl-5-allyl-1,3-dioxane-2-one (MAC).

(A) Synthesis scheme for 5-methyl-5-allyl-1,3-dioxane-2-one (MAC). Conditions: (1A) $\mathrm{KOH}, \mathrm{DMF}, 100^{\circ} \mathrm{C}$, $15 \mathrm{~h}$. (1B) Triphosgene, pyridine, $\mathrm{CH}_{2} \mathrm{Cl}_{2},-78-0^{\circ} \mathrm{C}$. (1C) $\mathrm{Pd} / \mathrm{C}$ (10\%), $\mathrm{H}_{2}$, ethyl acetate, RT, 40 psi, 3 h. (1D) EDC, HOBT, TEA, DMF, RT, 18 h. (2A) $\mathrm{KOH}, \mathrm{DMF}$, preheat to $100^{\circ} \mathrm{C}, 1 \mathrm{~h}, 45^{\circ} \mathrm{C}, 48 \mathrm{~h}$. (2B) $\mathrm{ClCO}_{2} \mathrm{Et}, \mathrm{THF}, \mathrm{TEA}, 0^{\circ} \mathrm{C}, 30 \mathrm{~min}$, RT, 12h. (B) ${ }^{1} \mathrm{H}$ NMR spectrum of $\mathrm{MAC}_{\text {in }} \mathrm{CDCl}_{3}$. (C) FT-IR spectrum of MAC. 


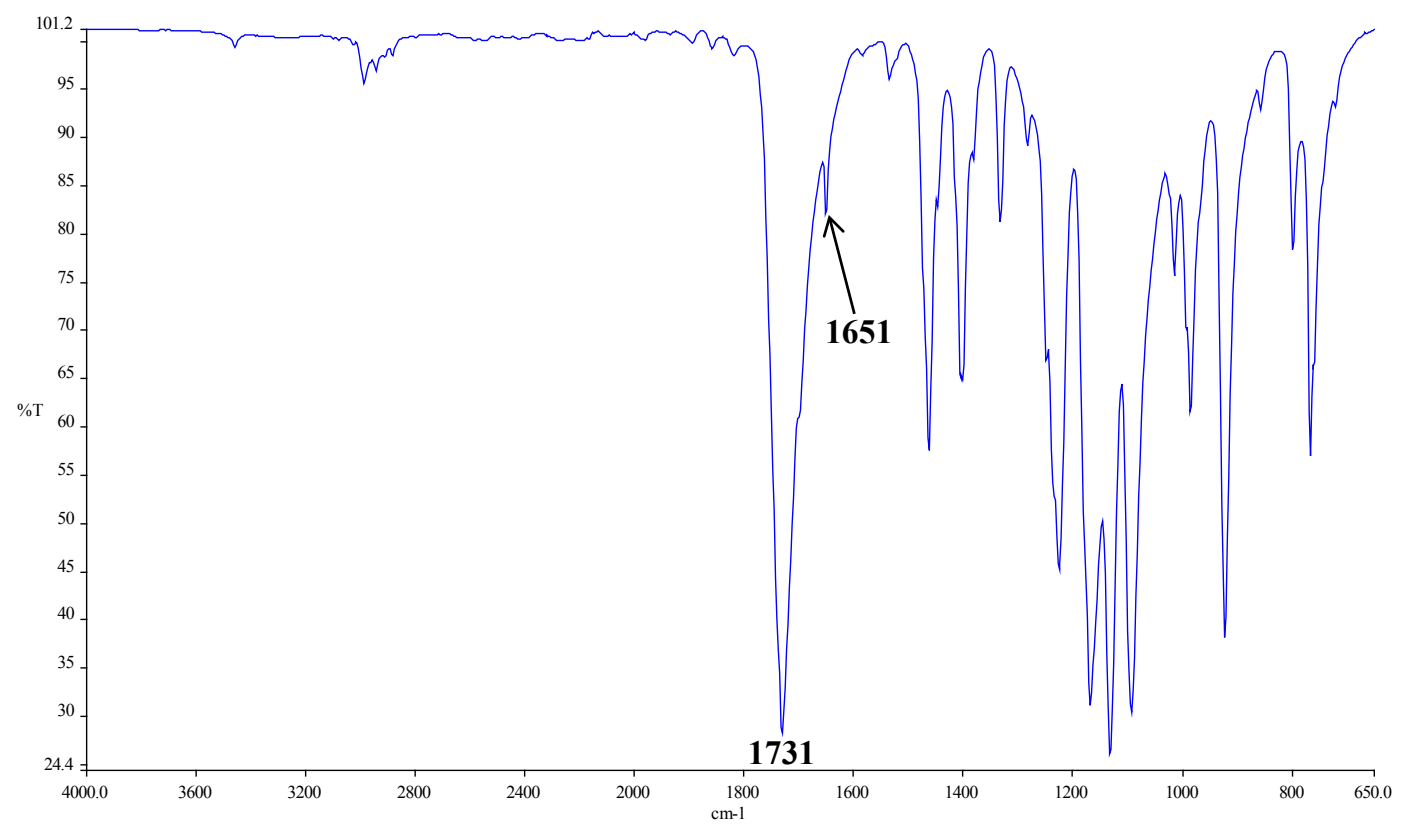

Figure 6-2. FT-IR spectrum of 5-methyl-5-allyl-1,3-dioxane-2-one (MAC). 


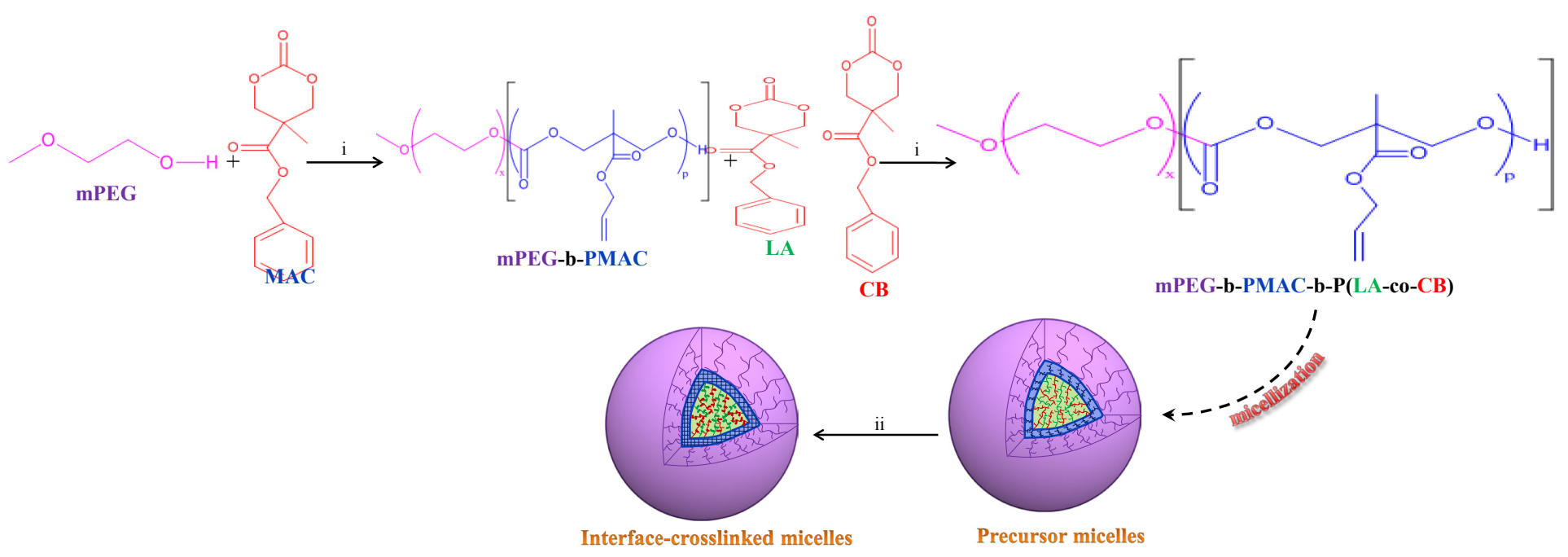

Figure 6-3. Synthesis method for preparation of interface-crosslinked micelles.

Conditions: (i) DBU, $\mathrm{CH} 2 \mathrm{Cl} 2, \mathrm{RT}, 3$ h. (ii) $\mathrm{AIBN}, 60^{\circ} \mathrm{C}$. 

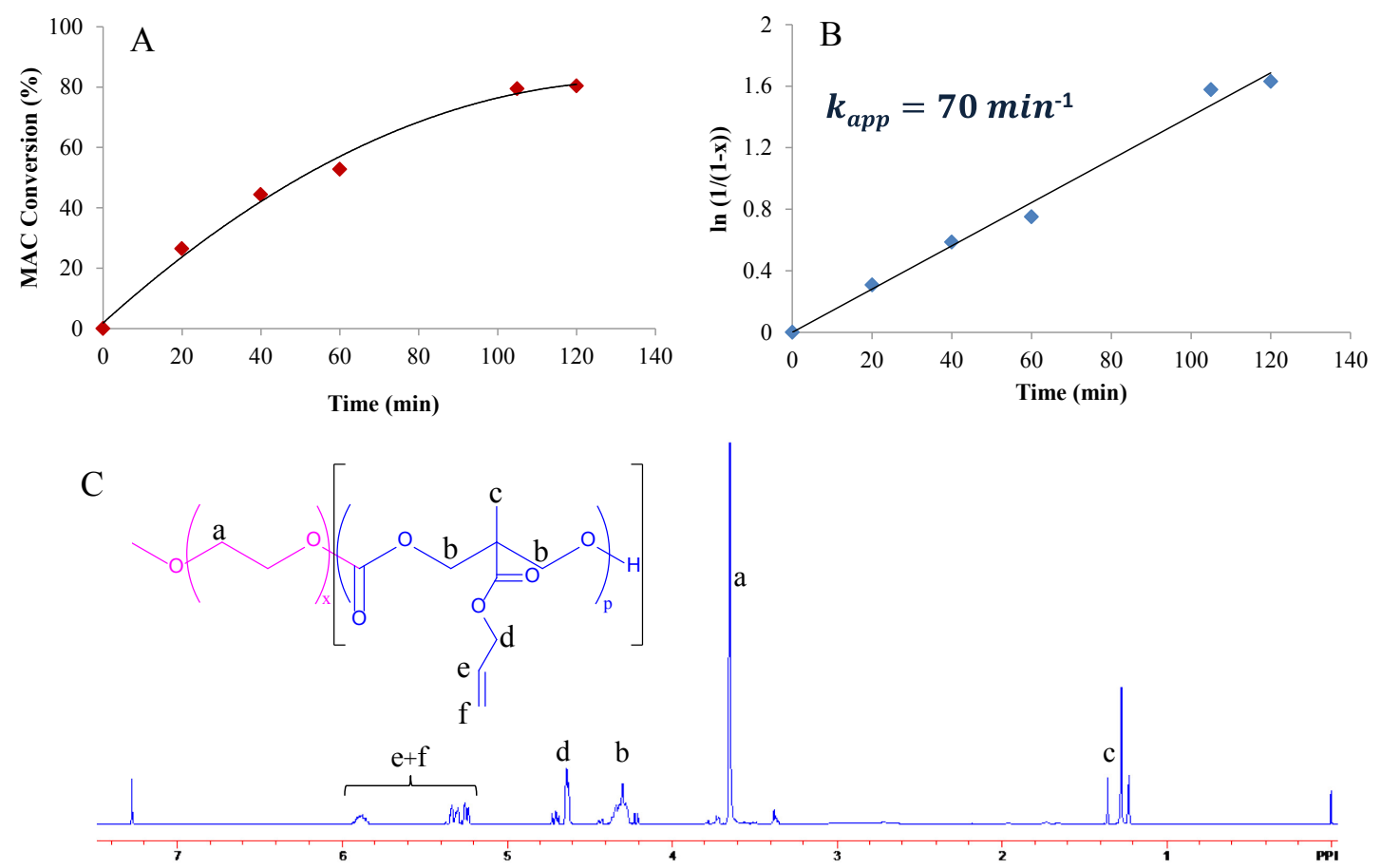

Figure 6-4. Methoxy poly (ethylene glycol) and MAC copolymerization in $\mathrm{CH2Cl2}$ at room temperature.

Reaction progress was monitored by $1 \mathrm{H}$ NMR spectroscopy. (A) Plot of $\ln (1 /(1-\mathrm{x}))$ vs. time ( $\mathrm{x}=$ monomer conversion); (B) Observed MAC conversion during synthesis of mPEG-b-PMAC copolymer. (C) 1H NMR spectrum of methoxy poly (ethylene glycol)b-poly (5-methyl-5-allyl-1,3-dioxane-2-one) (mPEG-b-PMAC) copolymer in CDCl3. 
when DBU amount was $5 \mu \mathrm{L} / \mathrm{mL}$. The observed conversion saturation could possibly be due to lower MAC concentrations. A plot of $\ln (1 /(1-\mathrm{x}))$ vs time resulted in a straight line with a 70 min-1 rate constant. Since MAC conversion remained constant post 105 min, PEG-b-PMAC polymerization was carried for $2 \mathrm{~h}$ and characterized using $1 \mathrm{H}$ NMR spectroscopy before the addition of CB and LA. A typical 1H NMR spectrum for mPEG$b$-PMAC copolymer is displayed in Figure 6-4C.

The successful synthesis of mPEG- $b$-PMAC-b-P(CB-co-LA) copolymer was confirmed using $1 \mathrm{H}$ NMR spectroscopy and GPC. The presence of characteristic resonance peaks for mPEG (3.65 ppm), LA (1.5-1.57, $5.12 \mathrm{ppm})$, CB (1.24, 4.25-4.35, $5.2,7.3 \mathrm{ppm})$ and MAC (4.2-4.4, 4.6, 5.3-5.8 ppm) clearly confirmed the co-occurrence of all the monomers (Figure 6-5A). Some characteristics of the synthesized mPEG- $b$ PMAC- $b-\mathrm{P}(\mathrm{CB}-c o-\mathrm{LA})$ copolymer are summarized in Table 6-1. Copolymer molecular weight was determined by comparative analysis of the $\mathrm{mPEG}$ (i.e., $\mathrm{CH} 2-\mathrm{CH} 2-$ at 3.65 ppm), lactide (i.e., $\mathrm{CH}$ at 5.12), $\mathrm{CB}$ (i.e., $\mathrm{C} 6 \mathrm{H} 5$ at $7.3 \mathrm{ppm})$ and $\mathrm{MAC}(-\mathrm{CH} 2-\mathrm{CH}=\mathrm{CH} 2$ at $5.8 \mathrm{ppm}$ ) characteristic proton peaks from $1 \mathrm{H}$ NMR spectrum. mPEG- $b$-PMAC- $b-$ $\mathrm{P}(\mathrm{CB} c o-\mathrm{LA})$ molecular weight was determined to be $10558 \mathrm{~g}$ mol-1 from 1H NMR spectrum. GPC measurements also confirmed successful copolymer synthesis and showed the resulting copolymer to have a narrow polydispersity of 1.08 and Mn of 9752 $\mathrm{g} \mathrm{mol}^{-1}$ co-LA) molecular weight was determined to be $10558 \mathrm{~g}$ mol-1 from $1 \mathrm{H}$ NMR spectrum. GPC measurements also confirmed successful copolymer synthesis and showed the resulting copolymer to have a narrow polydispersity of 1.08 and Mn of 9752 $\mathrm{g} \mathrm{mol}^{-1}$.

Synthesis and characterization of mPEG-b-P(CB-co-LA-co-MAC) copolymer

The synthetic procedure for mPEG- $b$-P(CB-co-LA-co-MAC) copolymer is shown in Figure 6-6. mPEG- $b-\mathrm{P}(\mathrm{CB}-c o-\mathrm{LA}-c o-\mathrm{MAC})$ copolymer was obtained by ring opening polymerization of $\mathrm{CB}, \mathrm{MAC}$ and $\mathrm{LA}$ using $\mathrm{PEO}$ as a macroinitiator and $\mathrm{DBU}$ as a catalyst. The monomer to initiator mole ratio was set at 20:1 with MAC monomer constituting $8 \mathrm{~mol} \%$. Addition of $5 \mathrm{~mol} \%$ DBU resulted in greater than $80 \%$ monomer conversion in $3 \mathrm{~h}$ affording copolymer with controlled molecular weights and low. polydispersity. 1H NMR spectrum (Figure 6-5B) of mPEG- $b$-P(CB-co-LA-co-MAC) copolymer showed the following resonance peaks at $\delta 3.65,5.12,5.28-5.8$ and 7.3 attributable to the methylene protons of $\mathrm{mPEG}$, the methylene protons of lactide, the acryloyl protons of MAC and the phenyl protons of carbonate, respectively. Furthermore, the signals at 4.25-4.35 reflect the methylene protons in the carbonate main chain and confirm successful ring opening polymerization of the carbonate monomers. The molecular weight of mPEG-b-P(CB-co-LA-co-MAC) copolymer was estimated to be $10020 \mathrm{~g} / \mathrm{mol}$ using the $\mathrm{mPEG}(\delta=3.65), \mathrm{LA}(\delta=5.12), \mathrm{CB}(\delta=7.3)$ and $\mathrm{MAC}(\delta=5.8)$ characteristic peaks. GPC revealed mPEG-b-P(CB-co-LA-co-MAC) copolymer to have a polydispersity of 1.08 and $\mathrm{Mn}$ of $10279 \mathrm{~g} / \mathrm{mol}$ which was close to the theoretical molecular weight and that determined by $1 \mathrm{H}$ NMR spectroscopy. 

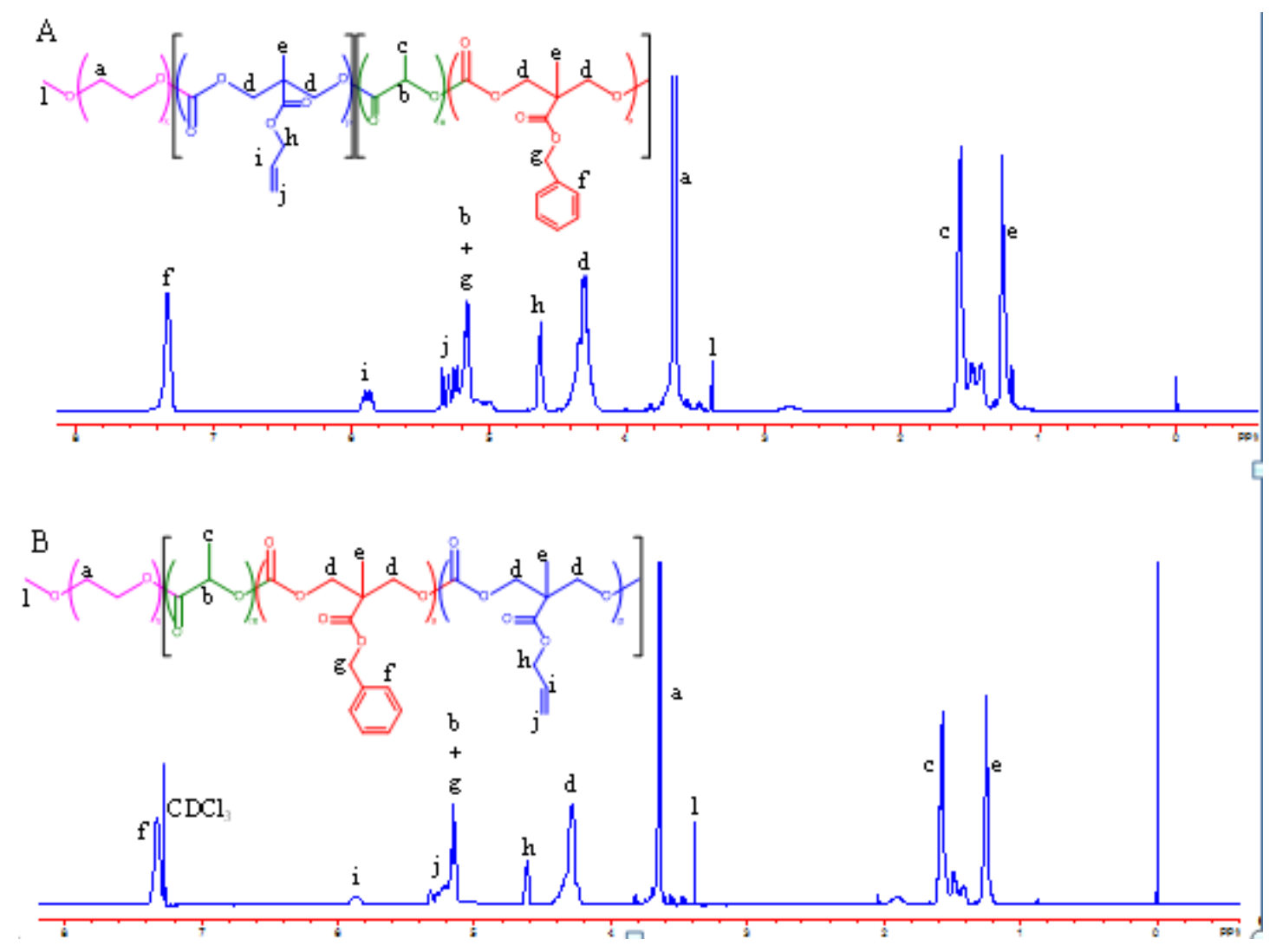

Figure 6-5. $\quad{ }^{1} \mathrm{H}$ NMR spectra for lactic acid and carbonate-based copolymer for crosslinked micelles.

(A) Methoxy poly (ethylene glycol)- $b$-poly (5-methyl-5-allyl-1,3-dioxane-2-one)- $b$ poly (carbonate-co-lactide) [mPEG- $b$-PMAC- $b-\mathrm{P}(\mathrm{CB}-c o-\mathrm{LA})$ ] copolymer in $\mathrm{CDCl}_{3}$. (B) Methoxy poly (ethylene glycol)- $b$-poly (5-methyl-5-allyl-1,3-dioxane-2-one-cocarbonate-co-lactide) [mPEG- $b$-P(CB-co-LA-co-MAC)] copolymer in $\mathrm{CDCl}_{3}$. 
Table 6-1. Characteristics of lactic acid and carbonate-based copolymers.

\begin{tabular}{llllll}
\hline Block copolymer $^{\mathbf{a}}$ & $\begin{array}{l}\mathbf{M}_{\mathbf{n}} \\
\left.\mathbf{(}_{\mathbf{1}} \mathbf{H} \mathbf{N M R}^{\mathbf{b}}\right)\end{array}$ & $\begin{array}{l}\mathbf{M}_{\mathbf{n}} \\
(\mathbf{G P C})\end{array}$ & $\begin{array}{l}\mathbf{M}_{\mathbf{w}} \\
\left.\mathbf{G P C}^{\mathbf{c}}\right)\end{array}$ & $\begin{array}{l}\mathbf{M}_{\mathbf{w}} / \mathbf{M}_{\mathbf{n}} \\
\left.\mathbf{( G P C}^{\mathbf{c}}\right)\end{array}$ & $\mathbf{C M C}(\mathbf{g} / \mathbf{L})$ \\
\hline $\mathrm{mPEG}_{114}-b-\mathrm{P}\left(\mathrm{CB}_{8}-c o-\mathrm{LA}_{35}-c o-\mathrm{MAC}_{2.5}\right)$ & 10020 & 10279 & 11103 & 1.08 & 0.001 \\
$\mathrm{mPEG}_{114}-b-\mathrm{PMAC}_{2.5}-b-\mathrm{P}\left(\mathrm{CB}_{9}-c o-\mathrm{LA}_{39}\right)$ & 10558 & 9752 & 10491 & 1.08 & 0.0008 \\
\hline
\end{tabular}

${ }^{a}$ Subscripts reflect degree of polymerization of each monomer obtained from ${ }^{1} \mathrm{H}$ NMR spectroscopy. ${ }^{\mathrm{b}}$ Molecular weight calculated from ${ }^{1} \mathrm{H}$ NMR spectroscopy in $\mathrm{g} / \mathrm{mol} .{ }^{\mathrm{c}}$ Molecular weight obtained from GPC in $\mathrm{g} / \mathrm{mol}$. 


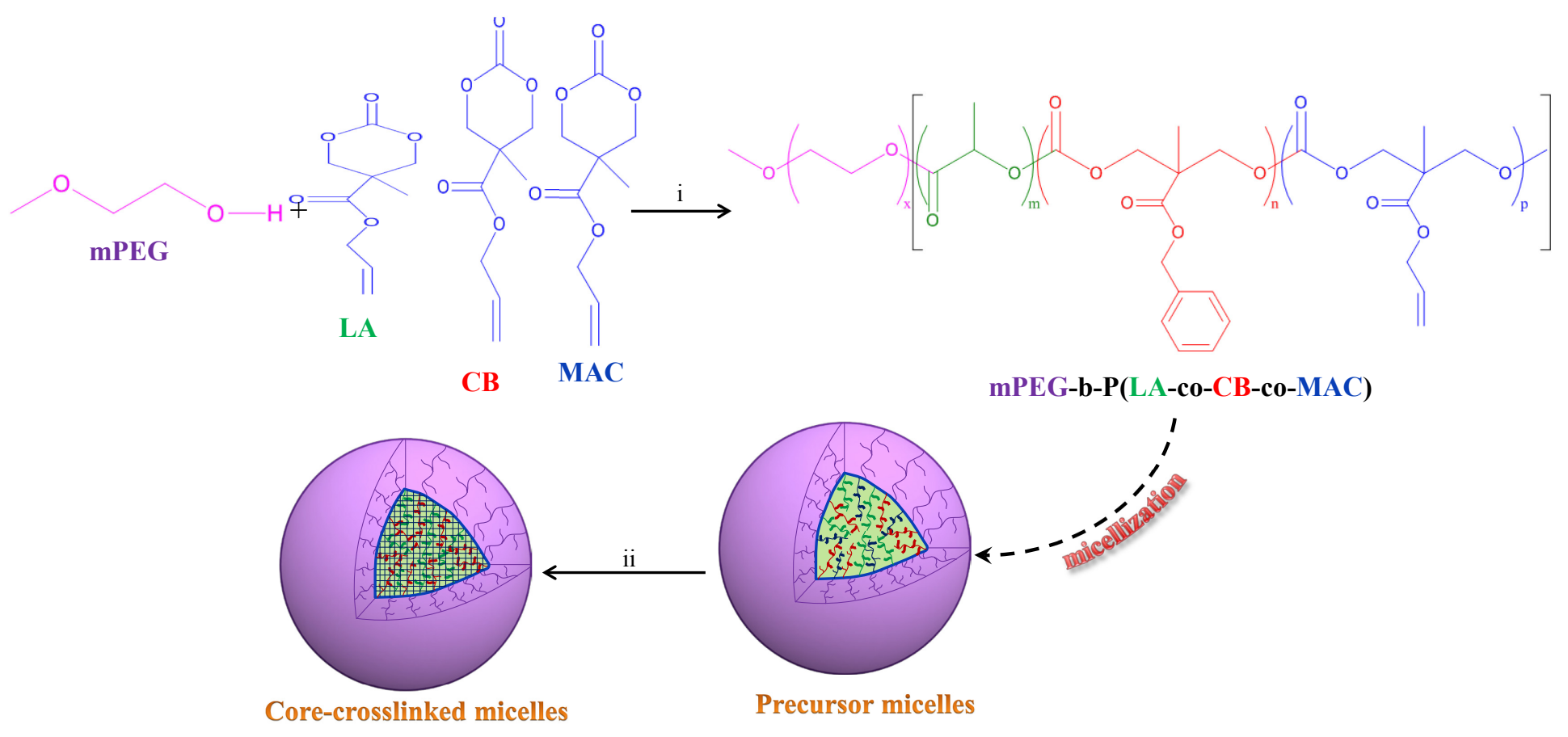

Figure 6-6. Synthesis method for preparation of lactic acid and carbonate-based crosslinked micelles.

Conditions: (i) DBU, $\mathrm{CH}_{2} \mathrm{Cl}_{2}$, RT, 3 h. (ii) $\mathrm{AIBN}, 60^{\circ} \mathrm{C}$. 
Microstructure analysis and polymer toxicity

MAC arrangement is expected to be blocky in mPEG- $b$-PMAC- $b-\mathrm{P}(\mathrm{CB}-c o-\mathrm{LA})$ and random in mPEG- $b$-P(CB-co-LA-co-MAC) copolymers. Since 13C NMR spectroscopy is sensitive to chemical shifts of the quaternary carbon in the polymer backbone and secondary carbon in the side group, it was used to confirm MAC arrangement in both copolymers. The $13 \mathrm{C}$ NMR spectra of mPEG-b-PMAC- $b-\mathrm{P}(\mathrm{CB}-\mathrm{co}-$ $\mathrm{LA})$ and mPEG- $b$-P(CB-co-LA-co-MAC) copolymers are shown in Figure 6-7A and $\mathbf{B}$, respectively, and further confirm successful copolymer synthesis. However, to determine MAC monomer arrangement, the 13C NMR spectra of mPEG- $b$-PMAC, mPEG- $b$ PMAC- $b$-P(CB- $c o$-LA), mPEG- $b$-P(CB- $c o$-LA- $c o$-MAC) and mPEG- $b$-P(CB-co-LA) copolymers were analyzed by observing peaks at $65.82 \mathrm{ppm}$ (secondary carbon) and 46.48 ppm (quaternary carbon), respectively.

From Figure 6-8, a distinctive peak can be observed at $65.82 \mathrm{ppm}$ for mPEG-bPMAC copolymer synonymous with the secondary carbon on the allyl group. This characteristic peak at $65.82 \mathrm{ppm}$ is also evident for mPEG- $b$-PMAC- $b$-P(CB-co-LA) copolymer suggesting presence of mPEG- $b$-PMAC block in mPEG- $b$-PMAC- $b$-P(CB-coLA) copolymer. The additional peak shifted downfield is assigned as the same carbon of MAC unit adjacent to $\mathrm{P}(\mathrm{CB}-\mathrm{co}-\mathrm{LA})$ which is reasonable since PMAC is short $(\mathrm{n}=2.5)$. In contrast, the characteristic secondary carbon peak of MAC shifts downfield for $\mathrm{mPEG}$ $b$ - $\mathrm{P}(\mathrm{CB}-\mathrm{co}$-LA-co-MAC) copolymer, indicating lack of MAC block sequence. This MAC peak is clearly absent in mPEG- $b$-P(CB-co-LA) copolymer. Furthermore, the 46.48 ppm peak representing the quaternary carbon in $\mathrm{mPEG}-b$-PMAC main chain is seen in the spectra of mPEG- $b$-PMAC-b-P(CB-co-LA) copolymer. The same peak is seen for mPEG- $b$-PMAC-b-P(CB-co-LA) overlapped with the broad peak which is corresponding to the quaternary carbon from the $\mathrm{CB}$ unit. Unlike mPEG- $b$-PMAC and mPEG- $b$-PMAC$b$-P(CB-co-LA), the 46.48 ppm peak is shifted downfield for mPEG- $b$-P(CB-co-LA-co$\mathrm{MAC}$ ) demonstrating random distribution of MAC monomer. A similar broad peak can be observed for mPEG- $b$ - $\mathrm{P}(\mathrm{CB}-\mathrm{co}-\mathrm{LA})$ reflecting contribution from the $\mathrm{CB}$ quaternary carbon. Since allyl compounds can be toxic, we evaluated the toxicity of mPEG- $b$ PMAC- $b$-P(CB-co-LA) and mPEG- $b$-P(CB-co-LA-co-MAC) copolymers in LNCaP prostate cancer cell lines. Our data reveal both copolymers are not toxic up to 2000 $\mu \mathrm{g} / \mathrm{mL}$ (Figure 6-9).

In contrast, the characteristic secondary carbon peak of MAC shifts downfield for mPEG- $b$-P(CB-co-LA-co-MAC) copolymer, indicating lack of MAC block sequence. This MAC peak is clearly absent in $\mathrm{mPEG}-b$-P(CB-co-LA) copolymer. Furthermore, the 46.48 ppm peak representing the quaternary carbon in $\mathrm{mPEG}-b$-PMAC main chain is seen in the spectra of mPEG- $b$-PMAC- $b-\mathrm{P}(\mathrm{CB}-c o-\mathrm{LA})$ copolymer. The same peak is seen for $\mathrm{mPEG}-b$-PMAC-b-P(CB-co-LA) overlapped with the broad peak which is corresponding to the quaternary carbon from the CB unit. Unlike mPEG- $b$-PMAC and mPEG- $b$-PMAC- $b$-P(CB-co-LA), the 46.48 ppm peak is shifted downfield for mPEG- $b$ $\mathrm{P}(\mathrm{CB}-\mathrm{co}-\mathrm{LA}-\mathrm{co}-\mathrm{MAC})$ demonstrating random distribution of MAC monomer. A similar broad peak can be observed for $\mathrm{mPEG}-b-\mathrm{P}(\mathrm{CB}-c o-\mathrm{LA})$ reflecting contribution from the $\mathrm{CB}$ quaternary carbon. Since allyl compounds can be toxic, we evaluated the toxicity of 


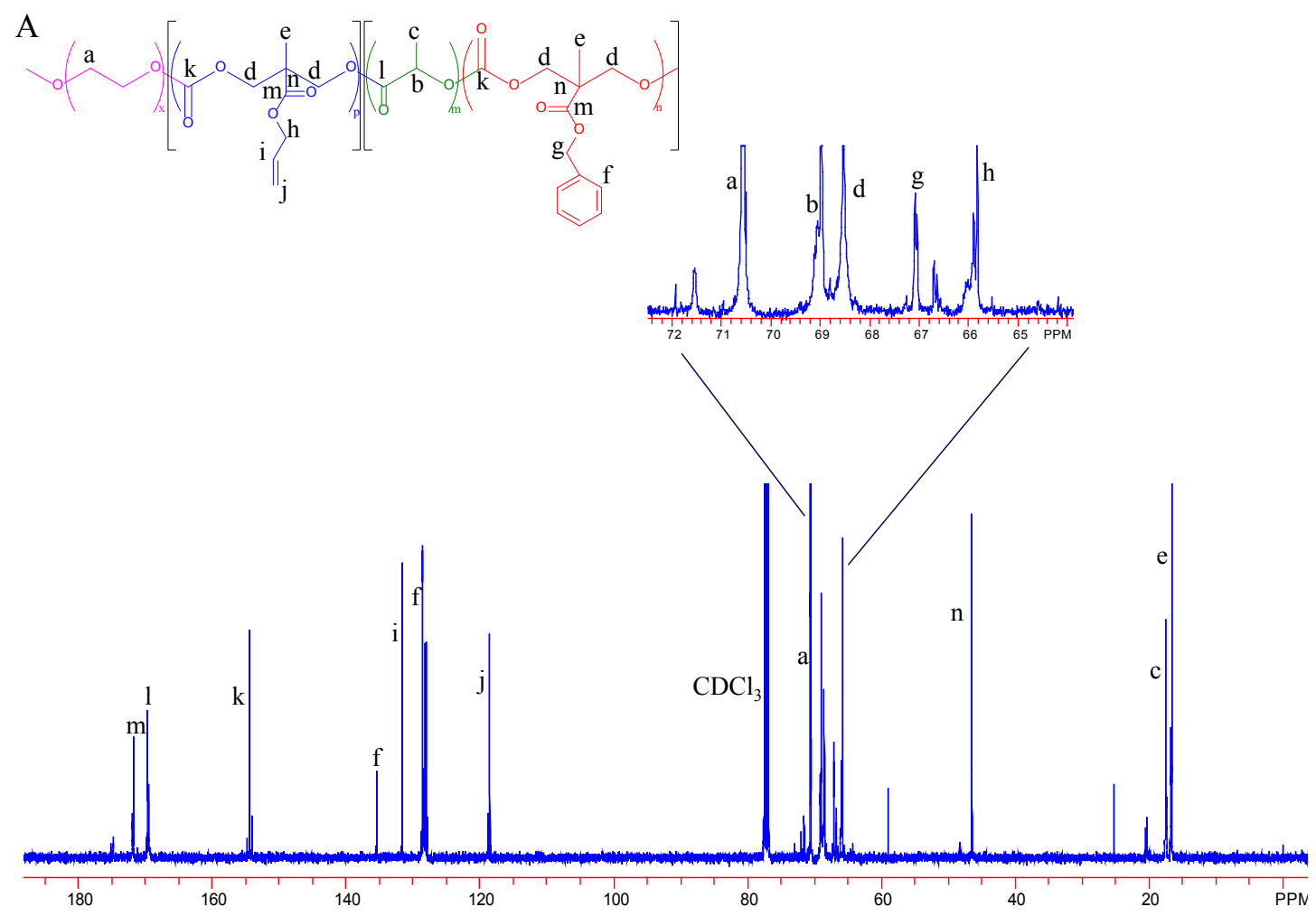

B

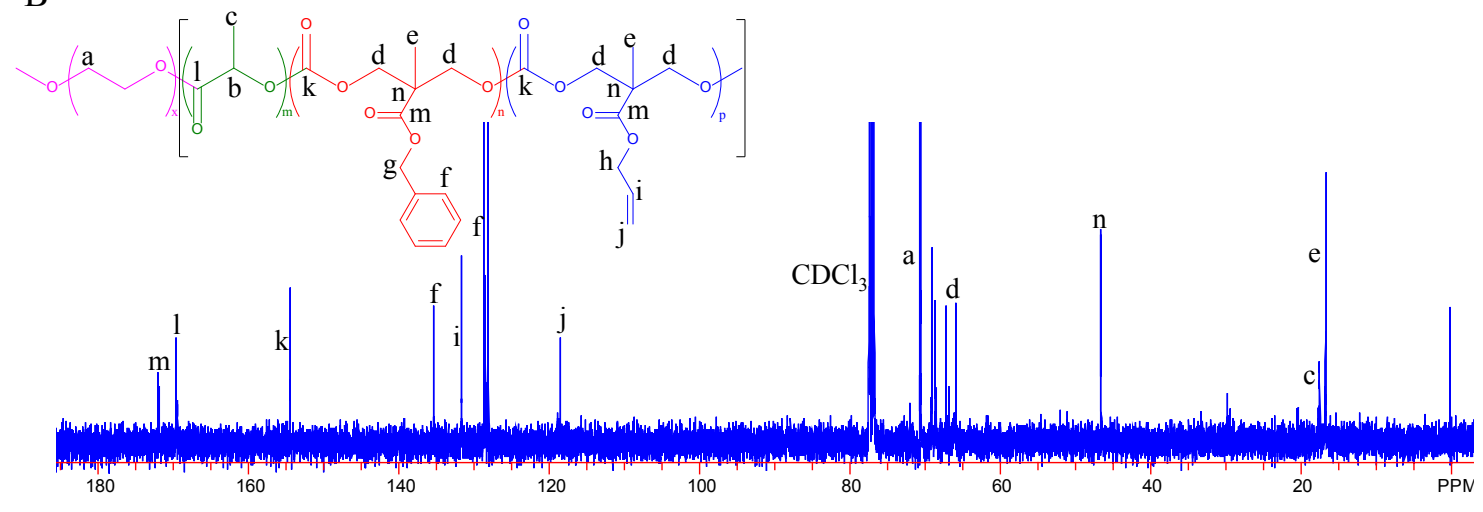

Figure 6-7. $\quad{ }^{13} \mathrm{C}$ NMR spectra for lactic acid and carbonate-based copolymer for crosslinked micelles.

(A) Methoxy poly (ethylene glycol)- $b$-poly (5-methyl-5-allyl-1,3-dioxane-2-one)- $b$ poly(carbonate-co-lactide) [mPEG- $b$-PMAC- $b$-P(CB-co-LA)] copolymer in $\mathrm{CDCl}_{3}$. (B) Methoxy poly (ethylene glycol)- $b$-poly (5-methyl-5-allyl-1,3-dioxane-2-one-cocarbonate-co-lactide) [mPEG- $b-\mathrm{P}(\mathrm{CB}-c o-\mathrm{LA}-c o-\mathrm{MAC})]$ copolymer in $\mathrm{CDCl}_{3}$. 

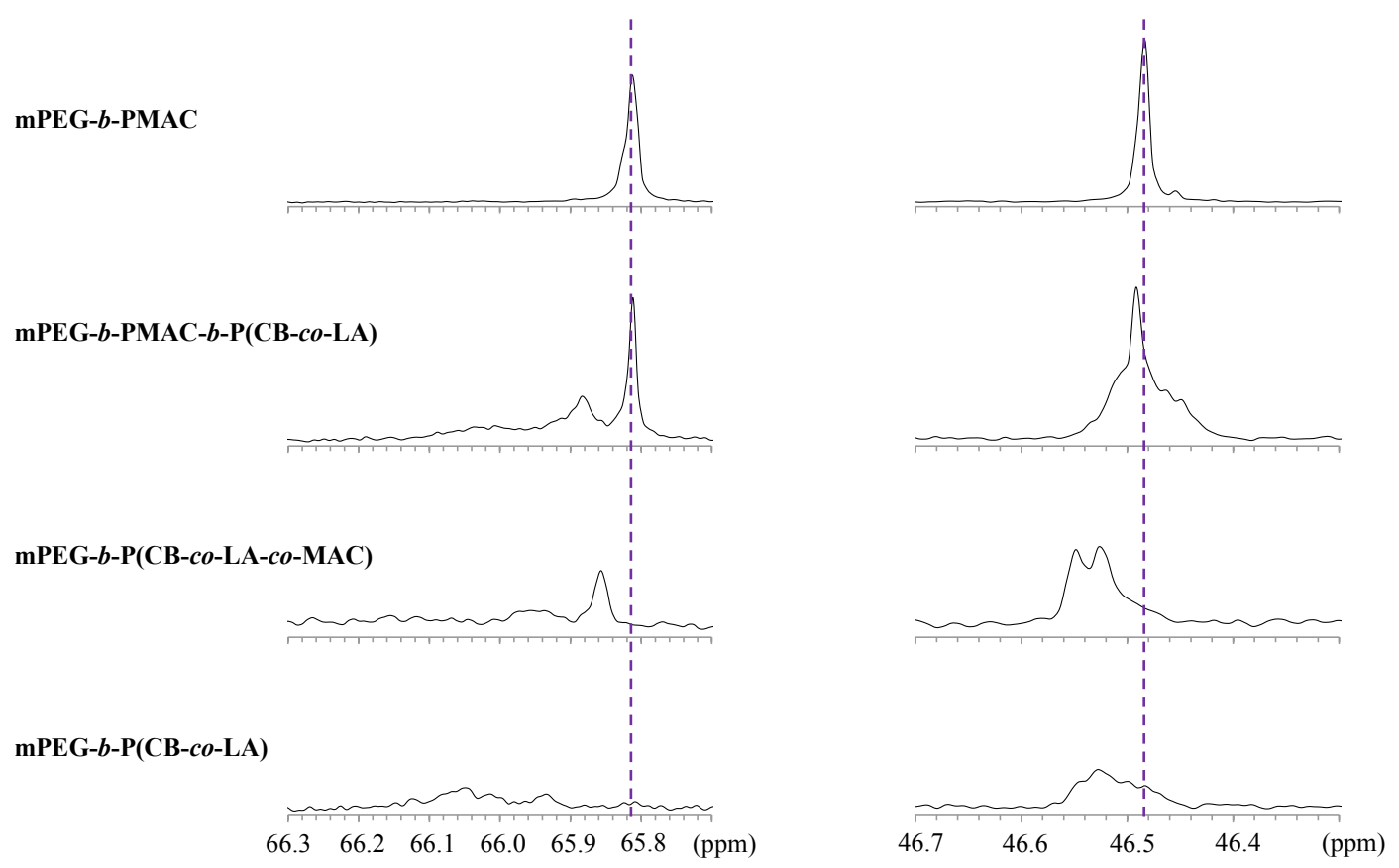

Figure 6-8. $\quad{ }^{13} \mathrm{C}$ NMR spectra comparative plot for mPEG- $b$-PMAC, mPEG- $b$ PMAC- $b$-P(CB-co-LA), mPEG- $b$-P(CB-co-LA-co-MAC) and mPEG- $b$-P(CB-co-LA) demonstrating differences in monomer arrangement. 


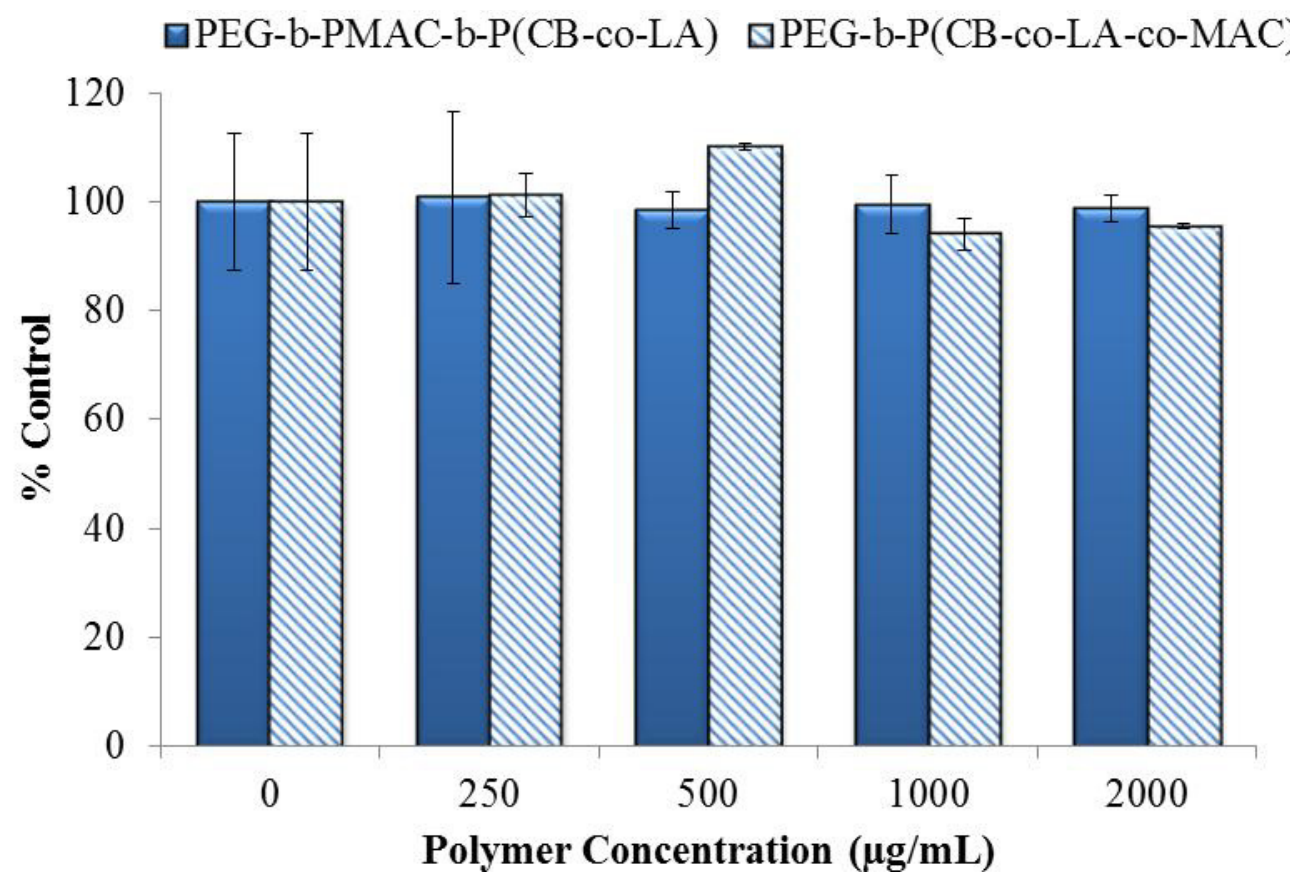

Figure 6-9. Cytotoxicity of mPEG-b-PMAC-b-P(CB-co-LA) and mPEG- $b$-P(CBco-LA-co-MAC) copolymers in prostate cancer cells.

LNCaP cells were incubated with various concentrations of copolymers $(0-2000$ $\mu \mathrm{g} / \mathrm{mL}$ ) for $48 \mathrm{~h}$ and cell viability determined by MTT assay. Data are presented as mean \pm standard deviation $(\mathrm{n}=3)$. 
mPEG- $b$-PMAC- $b$-P(CB-co-LA) and mPEG- $b$-P(CB-co-LA-co-MAC) copolymers in LNCaP prostate cancer cell lines. Our data reveal both copolymers are not toxic up to $2000 \mu \mathrm{g} / \mathrm{mL}$ (Figure 6-9).

Effect of polymer composition and MAC location on CMC, particle size and drug loading

Slight changes in polymer composition (e.g., monomer content and arrangement) can affect micelle properties. Therefore, we examined the effect of polymer composition, MAC location and crosslinking on CMC, size and drug loading. The CMC of mPEG- $b$ $\mathrm{P}(\mathrm{CB}-c o-\mathrm{LA}-c o-\mathrm{MAC})$ was found to be $0.001 \mathrm{~g} / \mathrm{L}$. In contrast mPEG- $b$-PMAC- $b$-P(CB$c o-\mathrm{LA}$ ) had a CMC of $0.0008 \mathrm{~g} / \mathrm{L}$. These results when juxtaposed with our previous findings [170] suggests the presence of the intermediate MAC block results in copolymers that form more thermodynamically stable micelles compared to random distribution of MAC in the hydrophobic core.

Subsequently, micelle size and drug-loading content were determined following preparation by the film sonication method. We observed the introduction of MAC monomer did not affect the micelle forming ability or morphology of the block copolymers regardless of its location. Micelle size and morphology were examined by dynamic light scattering (DLS) and transmission electron microscopy, respectively. Average hydrodynamic size was $123 \pm 3.02 \mathrm{~nm}$ for mPEG- $b$-P(CB-co-LA-co-MAC) copolymer while that of mPEG- $b$-PMAC- $b$-P(CB-co-LA) was $128 \pm 1.10 \mathrm{~nm}$ (Tables 6-2 and 6-3) with both copolymers resulting in micelles possessing low PDI values $(0.12-$ 0.15; reference Tables 6-2 and 6-3) implying a narrow micelle size distribution. Furthermore, our findings suggest inclusion of drug resulted in a modest increase in micelle size. Specifically, micelle size was $139 \pm 1.05 \mathrm{~nm}$ for mPEG- $b$-P(CB-co-LA-coMAC) copolymer while that of mPEG- $b$-PMAC- $b$-P(CB-co-LA) was $133 \pm 1.49 \mathrm{~nm}$. However, drug-loaded micelles still exhibited low PDI values.

We next quantified the amount of drug loaded into the micelles in terms of drug loading density (Equation 6-1) and encapsulation efficiency (Equation 6-2). From Table 6-2, mPEG- $b$-P(CB-co-LA-co-MAC) copolymer had superior drug loading characteristics compared to mPEG- $b$-PMAC- $b$-P(CB-co-LA). Based on a $5 \%$ theoretical loading, mPEG- $b$-P(CB-co-LA-co-MAC) had a drug loading density of $3.79 \% \pm 0.56$ indicating an encapsulation efficiency of $78.72 \% \pm 0.15$. In contrast, drug loading density for $\mathrm{mPEG}-b$-PMAC- $b$-P(CB-co-LA) was computed to be $1.01 \% \pm 0.73$ reflecting a $20.29 \% \pm 0.11$ encapsulation efficiency. Furthermore, surface charge of the micelles prepared from both copolymers were observed to be slightly negative: $-0.96 \pm 0.05$ and $5.52 \pm 0.13 \mathrm{mV}$ for mPEG-b-P(CB-co-LA- $c o$-MAC) and mPEG- $b$-PMAC- $b$-P(CB-coLA), respectively. 
Table 6-2. Effect of polymer composition and MAC location on particle size and drug loading in noncrosslinked micelles.

\begin{tabular}{|c|c|c|c|c|}
\hline Block copolymer $^{\text {a }}$ & Size $(\mathbf{n m})^{\text {b }}$ & PDI & $\begin{array}{l}\text { Drug loading } \\
(\%) \pm \\
\text { SD }^{\mathbf{c}}\end{array}$ & $\begin{array}{l}\text { Encapsulation } \\
\text { efficiency }(\%) \pm \\
\text { SD }\end{array}$ \\
\hline \multicolumn{5}{|l|}{ Before Drug Loading } \\
\hline $\mathrm{mPEG}_{114}-\mathrm{b}-\mathrm{P}\left(\mathrm{CB}_{8}-\mathrm{co}-\mathrm{LA}_{35}-\mathrm{co}-\mathrm{MAC}_{2.5}\right)$ & $123.0 \pm 3.02$ & $0.15 \pm 0.01$ & - & - \\
\hline $\mathrm{mPEG}_{114}-\mathrm{b}-\mathrm{PMAC}_{2.5}-\mathrm{b}-\mathrm{P}\left(\mathrm{CB}_{9}-\mathrm{co}-\mathrm{LA}_{39}\right)$ & $128.0 \pm 1.10$ & $0.12 \pm 0.01$ & - & - \\
\hline \multicolumn{5}{|l|}{ After Drug Loading } \\
\hline $\mathrm{mPEG}_{114}-\mathrm{b}-\mathrm{P}\left(\mathrm{CB}_{8}-\mathrm{co}-\mathrm{LA}_{35}\right.$-co-MAC 2.5$)$ & $139.0 \pm 1.05$ & $0.10 \pm 0.02$ & $3.79 \pm 0.56$ & $75.72 \pm 0.15$ \\
\hline $\mathrm{mPEG}_{114}-\mathrm{b}-\mathrm{PMAC}_{2.5}-\mathrm{b}-\mathrm{P}\left(\mathrm{CB}_{9}-\mathrm{co}-\mathrm{LA}_{39}\right)$ & $133.0 \pm 1.49$ & $0.04 \pm 0.01$ & $1.01 \pm 0.73$ & $20.29 \pm 0.11$ \\
\hline
\end{tabular}

${ }^{a}$ Subscripts reflect degree of polymerization of each monomer obtained from $1 \mathrm{H}$ NMR spectroscopy. ${ }^{\mathrm{b}}$ Mean particle size was determined by dynamic light scattering. ${ }^{\mathrm{c}}$ Percentage of drug loaded into micelles based on $5 \%$ theoretical loading. 
Table 6-3. Effect of polymer composition and MAC location on particle size and drug loading in crosslinked micelles.

\begin{tabular}{|c|c|c|c|c|}
\hline Block copolymer $^{\text {a }}$ & Size $(n m)^{b}$ & PDI & $\begin{array}{l}\text { Drug loading } \\
(\%) \pm \text { SD }^{c}\end{array}$ & $\begin{array}{l}\text { Encapsulation } \\
\text { efficiency }(\%) \pm \\
\text { SD }\end{array}$ \\
\hline \multicolumn{5}{|l|}{ Before Drug Loading } \\
\hline $\mathrm{mPEG}_{114}-\mathrm{b}-\mathrm{P}\left(\mathrm{CB}_{8}-\mathrm{co}-\mathrm{LA}_{35}-\mathrm{co}-\mathrm{MAC}_{2.5}\right)$ & $151.0 \pm 1.69$ & $0.12 \pm 0.04$ & - & - \\
\hline $\mathrm{mPEG}_{114}$-b-PMAC ${ }_{2.5}-\mathrm{b}-\mathrm{P}\left(\mathrm{CB}_{9}-\mathrm{co}-\mathrm{LA}_{39}\right)$ & $135.0 \pm 1.89$ & $0.20 \pm 0.03$ & - & - \\
\hline \multicolumn{5}{|l|}{ After Drug Loading } \\
\hline $\mathrm{mPEG}_{114}-\mathrm{b}-\mathrm{P}\left(\mathrm{CB}_{8}-\mathrm{co}-\mathrm{LA}_{35}-\mathrm{co}-\mathrm{MAC}_{2.5}\right)$ & $118.0 \pm 1.70$ & $0.10 \pm 0.01$ & $3.21 \pm 2.11$ & $64.08 \pm 0.48$ \\
\hline $\mathrm{mPEG}_{114}-\mathrm{b}-\mathrm{PMAC} \mathrm{C}_{2.5}-\mathrm{b}-\mathrm{P}\left(\mathrm{CB}_{9}-\mathrm{co}-\mathrm{LA}_{39}\right)$ & $122.0 \pm 0.81$ & $0.12 \pm 0.01$ & $0.89 \pm 0.10$ & $17.77 \pm 0.02$ \\
\hline
\end{tabular}

${ }^{a}$ Subscripts reflect degree of polymerization of each monomer obtained from 1H NMR spectroscopy. ${ }^{\mathrm{b}}$ Mean particle size was determined by dynamic light scattering. ${ }^{\mathrm{c}}$ Percentage of drug loaded into micelles based on $5 \%$ theoretical loading. 
Effect of crosslinking on particle size, morphology and drug loading

Next, mPEG- $b$-P(CB-co-LA-co-MAC) and mPEG- $b$-PMAC- $b$-P(CB-co-LA) copolymers were suitably crosslinked in a mixture of THF and water at $70^{\circ} \mathrm{C}$ using AIBN as the initiator. Pendant allyl moieties in the core and interface of copolymers allowed convenient crosslinking in the presence of AIBN initiator. ${ }^{1} \mathrm{H}$ NMR and IR spectroscopy was used to confirm the success of crosslinking. As shown in Figure 6-10A, a prominent peak at $\delta 5.8 \mathrm{ppm}$ (acryloyl proton) can clearly be seen in the non-crosslinked micelles. In contrast, the signal significantly weakened for the crosslinked sample (Figure 6-10B) together with the methylene protons of allyl group at $4.6 \mathrm{ppm}$. Quantitative analysis from the ${ }^{1} \mathrm{H}$ NMR spectra revealed $69 \%$ crosslinking efficiency. IR spectroscopy also confirmed successful crosslinking as indicated by the weakening of the $1650 \mathrm{~cm}^{-1}$ peak corresponding to the alkene group (Figure 6-11).

From our studies, crosslinking of non-drug loaded micelles generally led to an increase in micelle size compared to their non-crosslinked counterparts (Table 6-2). This size change was greater for mPEG- $b$-P(CB-co-LA-co-MAC) which increased from $123 \pm$ $3.02 \mathrm{~nm}$ to $151 \pm 1.69 \mathrm{~nm}$ compared to mPEG- $b$-PMAC- $b$-P(CB-co-LA) where size was $128 \pm 1.10 \mathrm{~nm}$ and $135 \pm 1.89 \mathrm{~nm}$ before and after crosslinking, respectively. Interestingly, we observed crosslinking of drug loaded micelles resulted in a decrease in size compared to their non-crosslinked counterparts (Tables 6-2 and 6-3). Here again, the size change was greater for mPEG- $b$-P(CB-co-LA-co-MAC) compared to mPEG- $b$ PMAC- $b$-P(CB-co-LA) $(21 \mathrm{~nm}$ vs. $11 \mathrm{~nm})$. Our findings also suggest crosslinking resulted in a modest decrease in drug loading regardless of copolymer type. Furthermore, TEM analysis demonstrates that crosslinking does not significantly alter micelle morphology (Figure 6-12).

\section{Effect of crosslinking on stability}

After confirming successful crosslinking of micelles, we next examined the effect of crosslinking on micelle stability in physiologically relevant and extreme micelle destabilization conditions. Since micelles encounter sink like conditions upon systemic administration, we first examined the stability of non-crosslinked and crosslinked micelles in PBS at micelle concentrations of 200, 20, 2 and $0.20 \mu \mathrm{g} / \mathrm{mL}$. Both noncrosslinked micelles (NCM) and crosslinked micelles (CM) remained intact up to 2 $\mu \mathrm{g} / \mathrm{mL}$ micelle concentration further demonstrating the thermodynamic stability of both micelle systems. Under these conditions, non-crosslinked micelles had an average size of $123 \pm 3.02 \mathrm{~nm}$ with $0.15 \pm 0.01$ PDI while crosslinked micelles exhibited an average size of $127 \pm 3.69 \mathrm{~nm}$ with $0.16 \pm 0.02 \mathrm{PDI}$. However, the non-crosslinked micelles dissociated when micelle concentration was $0.20 \mu \mathrm{g} / \mathrm{mL}$, whereas the crosslinked micelles maintained their nanostructure (Figure 6-13A).

We next investigated the stability of micelles with or without drug in the presence of serum for 24 and 48 h. From Figure 6-13B, non-crosslinked micelles (NCM) appeared to aggregate with time as indicated by increase in particle size. For example, the $\mathrm{d}_{\mathrm{i}} / \mathrm{d}_{0}$ 

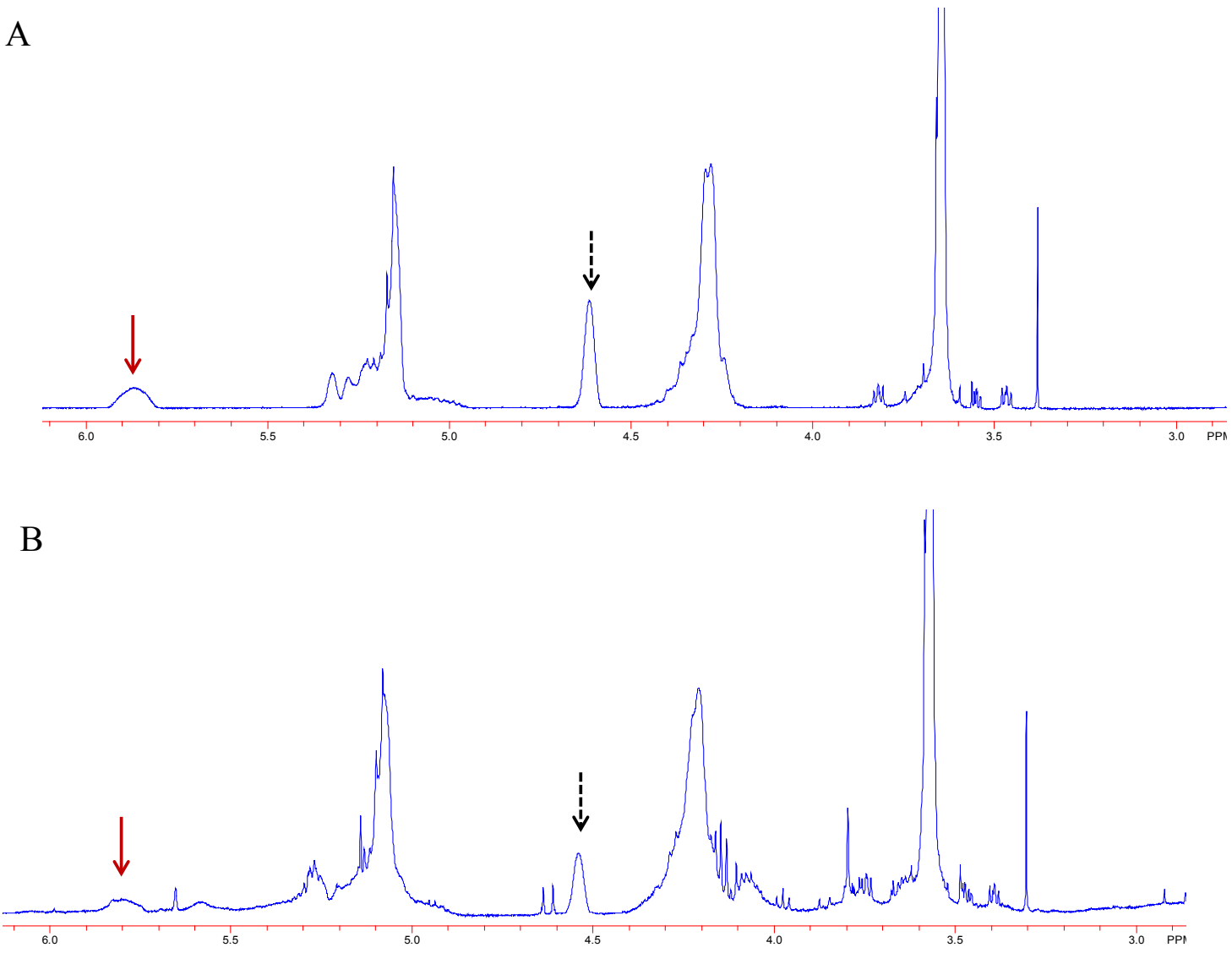

Figure 6-10. ${ }^{1}$ H NMR spectra for methoxy poly (ethylene glycol)-b-poly (5-methyl5-allyl-1,3-dioxane-2-one-co-carbonate-co-lactide) [mPEG-b-P(MAC-co-CB-co-LA)] copolymer in $\mathrm{CDCl}_{3}$.

(A) Before crosslinking and (B) after crosslinking. 

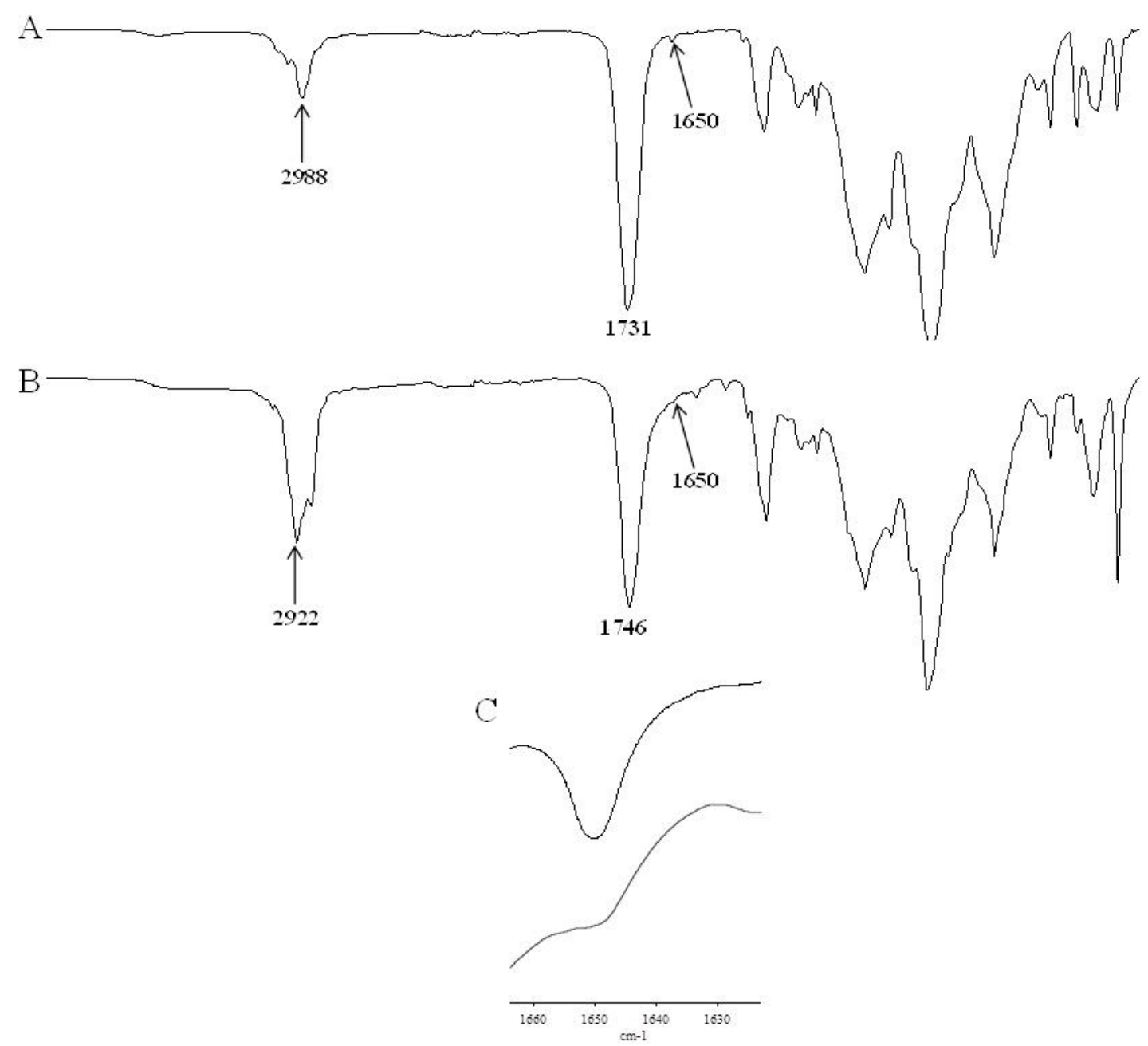

Figure 6-11. FTIR spectra for lactic acid and carbonate-based copolymer.

(A) Before crosslinking and (B) after crosslinking. Arrow indicates weakening of 1650 $\mathrm{cm}^{-1}$ peak reflecting crosslinking of double bonds. (C) Peaks at $1650 \mathrm{~cm}^{-1}$ zoomed in and offset for clarity; Top (before crosslinking) and Bottom (after crosslinking). 

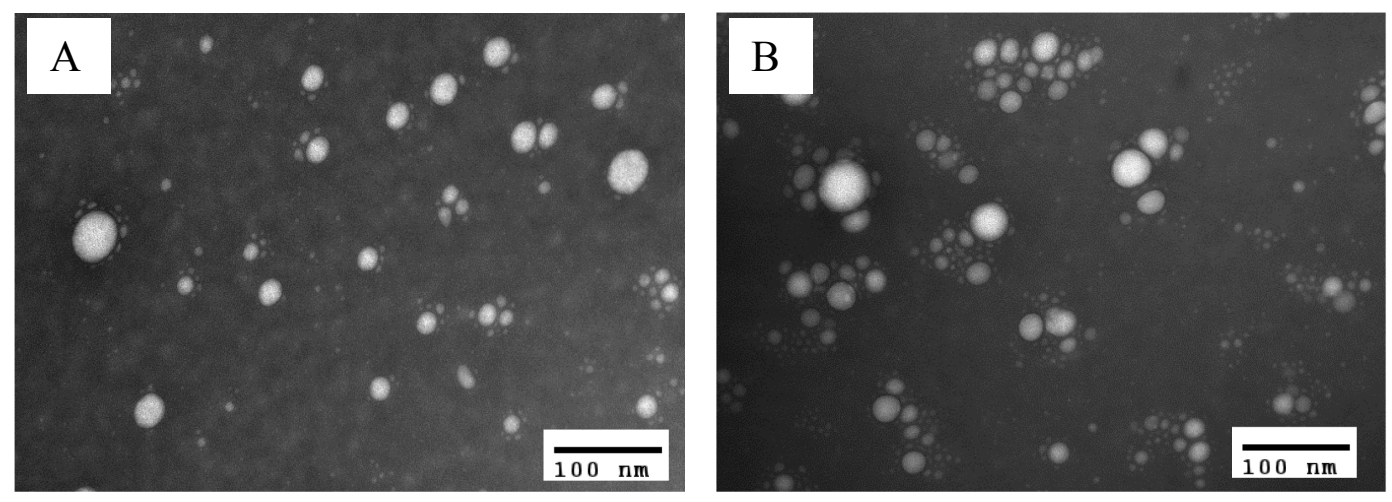

Figure 6-12. TEM images of (A) non-crosslinked and (B) crosslinked micelles. 

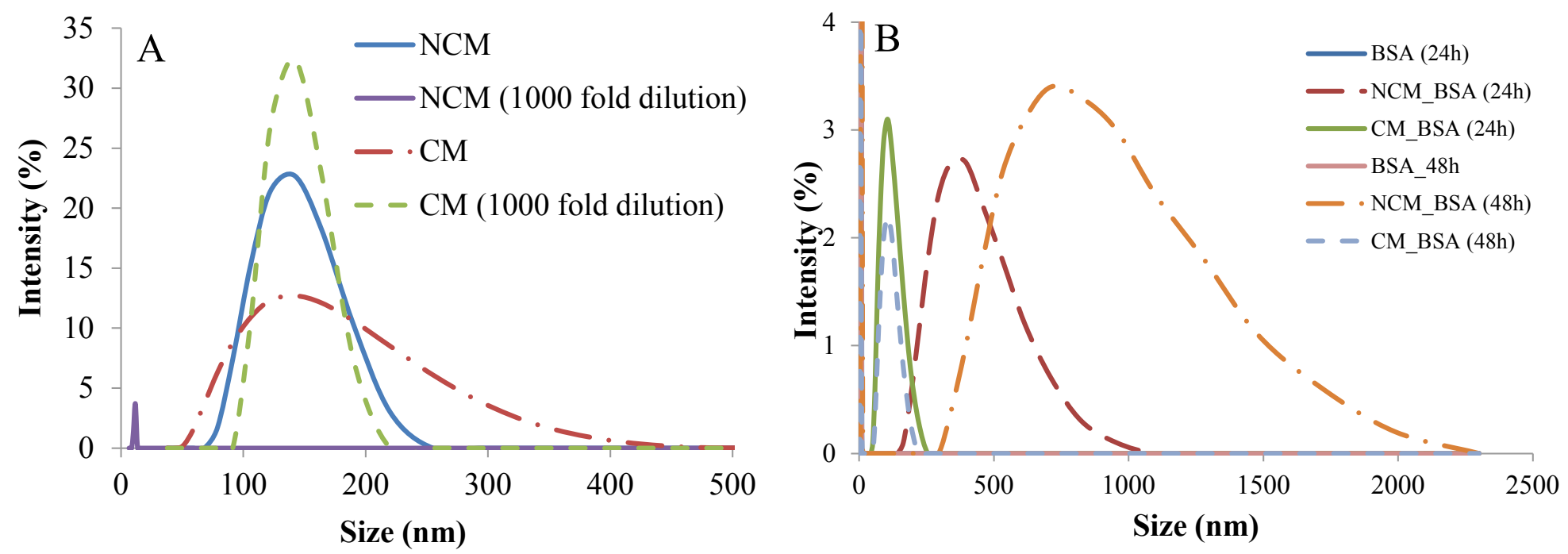

Figure 6-13. Stability of mPEG-b-PMAC-b-P(CB-co-LA) non-crosslinked (NCM) and crosslinked micelles (CM).

(A) Micelle stability against 1000 fold dilution. (B) Micelle stability in bovine serum albumin (BSA) after 24 and 48 h. 
(diameter at specified time to diameter at $\mathrm{t}=0$ ) ratio increased from approximately 1 to 4 and 8 at 24 and $48 \mathrm{~h}$, respectively. We also determined the CMC of $\mathrm{mPEG}-b-\mathrm{P}(\mathrm{CB}-\mathrm{co}-$ LA-co-MAC) non-crosslinked micelle and crosslinked micelles as a secondary measure of stability. Our results reveal uncrosslinked micelles had a CMC value of $1 \mathrm{mg} / \mathrm{L}$ based on where the two tangent lines intersect. In contrast, CMC of crosslinked micelles was determined to be $0.06 \mathrm{mg} / \mathrm{L}$. These results demonstrate crosslinked micelles enhanced stability compared to uncrosslinked micelles.

In vitro cytotoxicity of mPEG-b-PMAC-b-P(CB-co-LA) and mPEG-b-P(CB-co-LA-co$\mathrm{MAC}$ ) non-crosslinked (NCM) and crosslinked micelles (CM)

The inhibitory effect of bicalutamide-loaded mPEG- $b$-PMAC- $b$-P(CB-co-LA) and mPEG- $b$-P(CB-co-LA-co-MAC) non-crosslinked (NCM) and crosslinked micelles (CM) micelles was determined in LNCaP human prostate cancer cell line for $24 \mathrm{~h}$. From Figure 6-14, bicalutamide-loaded crosslinked micelles more potently inhibited LNCaP cell growth compared to bicalutamide-loaded non-crosslinked micelles regardless of polymer type. For bicalutamide-loaded mPEG- $b$-PMAC- $b$-P(CB-co-LA) micelles, $\mathrm{IC}_{50}$ for non-crosslinked micelles was $\sim 47.2 \mu \mathrm{M}$ while that of crosslinked micelles was $\sim 15.0$ $\mu \mathrm{M}$. Similarly, bicalutamide-loaded mPEG- $b$-P(CB-co-LA-co-MAC) non-crosslinked micelles had an $\mathrm{IC}_{50}$ of $\sim 36.9 \mu \mathrm{M}$ whilst crosslinked micelles had $\mathrm{IC}_{50}$ of $\sim 15.4 \mu \mathrm{M}$. Bicalutamide is known to affect expression of prostate specific antigen (PSA) which is downstream of and regulated by the androgen-androgen receptor signaling axis. Therefore, we examined the effect of bicalutamide-loaded crosslinked and noncrosslinked micelles on secreted PSA following incubation in LNCaP cells for $24 \mathrm{~h}$. Our results suggest that bicalutamide micelles reduce secreted PSA expression by approximately $50 \%$ compared to control, irrespective of polymer type and presence of crosslinking (Figure 6-15).

\section{Discussion}

Polymeric micelles are promising drug delivery platforms [11, 170, 247, 279281]. Insight accruing from micelle research particularly in oncology has led to the strategic design and synthesis of novel polymers to address the key issues of adequate drug loading, targetability, sustained drug release and degradation. Despite significant advances made in these areas, one reason the pharmaceutical industry has not fully adopted micelle formulations is their perceived instability especially in biological environments. The purpose of our study is to address this pertinent issue through the synthesis, characterization and in vitro evaluation of innovative lactic acid and carbonate based crosslinked micelles for enhanced drug delivery.

We have successfully synthesized $\mathrm{mPEG}_{114}-b-\mathrm{PMAC}_{2.5}-\mathrm{b}-\mathrm{P}\left(\mathrm{CB}_{9}-c o-\mathrm{L}_{\mathrm{A} 39}\right)$ and $\mathrm{mPEG}_{114}-b-\mathrm{P}\left(\mathrm{CB} 8-c o-\mathrm{LA}_{35}-c o-\mathrm{MAC}_{2.5}\right)$ copolymers for core-corona interface crosslinked and core crosslinked micelles, respectively (Figure 6-3). The design of these copolymers was guided by our desire to improve upon the existing micelle properties 


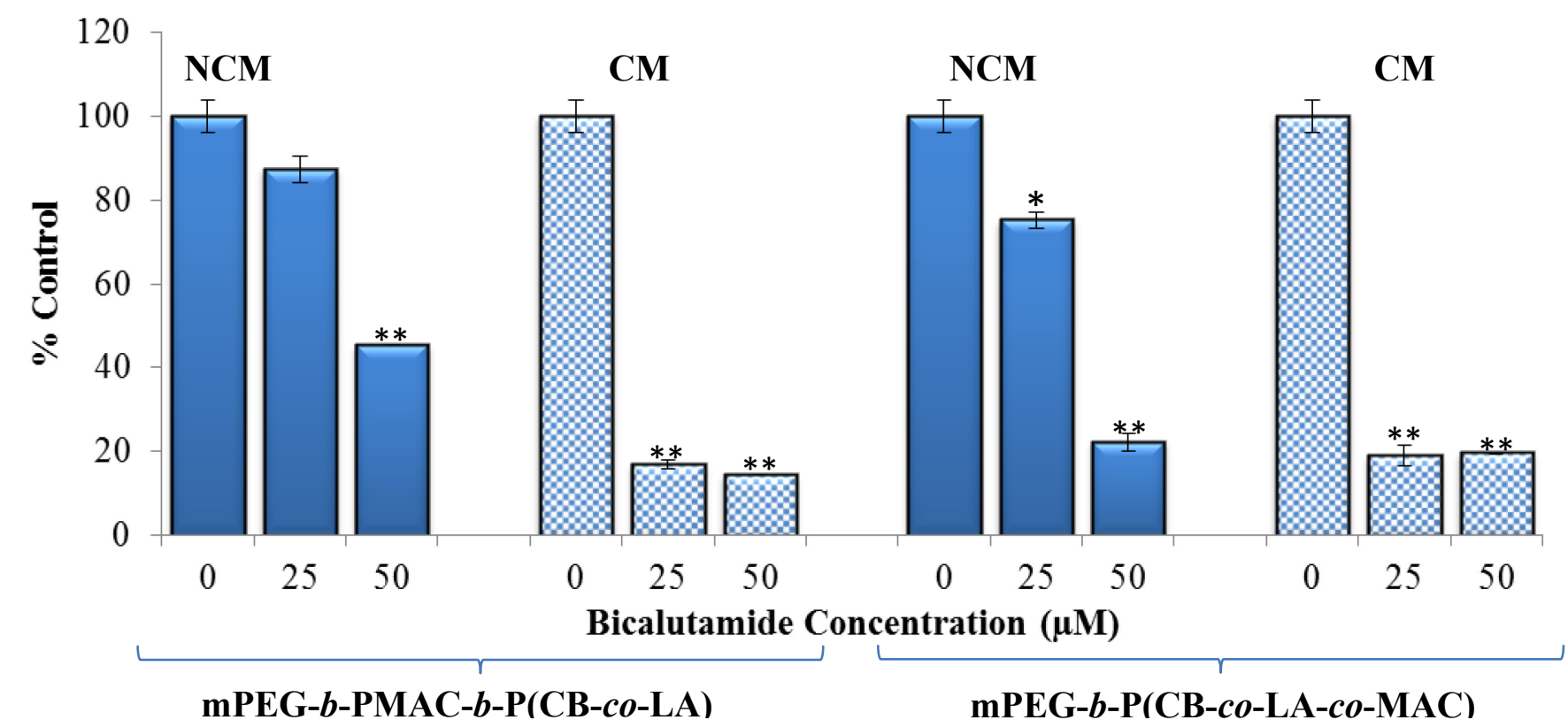

Figure 6-14. Anticancer effect of mPEG-b-PMAC-b-P(CB-co-LA) and mPeg- $b$-P(CB-so-LA-co-MAC) non-crosslinked (NCM) and crosslinked micelles (CM).

$3 \times 10^{3}$ LNCaP cells were incubated with bicalutamide-loaded micelle formulations $(0,25$ and $50 \mu \mathrm{M}$ of drug) for $24 \mathrm{~h}$. Cell viability was determined using MTT assay and results represented as the mean $\pm \mathrm{SD}$ of triplicates. ${ }^{*} \mathrm{p}<0.05 ; * * \mathrm{p}<0.01$ using student's unpaired t-test. 


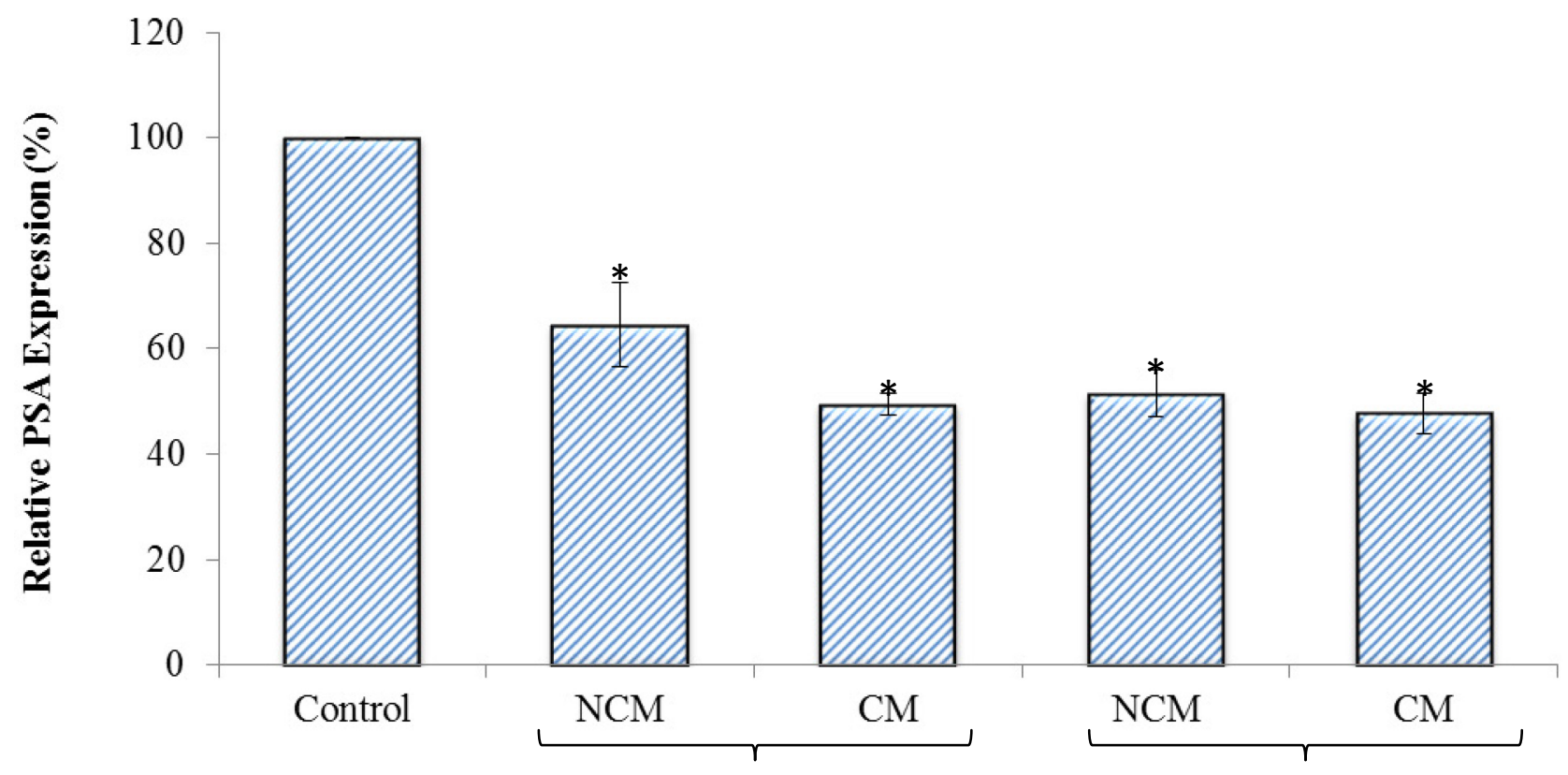

PEG-b-PMAC-b-P(CB-co-LA) PEG-b-P(CB-co-LA-co-MAC)

Figure 6-15. Effect of bicalutamide-loaded mPEG- $b$-PMAC- $b-P(C B-c o-L A)$ and mPEG- $b$-P(CB-co-LA-co-MAC) non-crosslinked (NCM) and crosslinked micelles (CM) (50 $\mu \mathrm{M}$ of drug) on secreted prostate specific antigen (PSA) following $24 \mathrm{~h}$ incubation.

$* \mathrm{p}<.05$ using student's unpaired t-test. 
obtained using our previously synthesized mPEG- $b$-P(CB-co-LA) copolymers, especially with regards to sub $\mathrm{CMC}$ and serum stability. Therefore, we decided to strategically introduce chemical crosslinks at the core-corona interface with the goal of creating a molecular fence to improve micelle integrity and sustained release. We also introduced crosslinks in the core as a complimentary approach and to demonstrate the flexibility of our crosslinking approach. Although a variety of chemistries (e.g., click chemistry, disulfide bond) can be used for crosslinking, we judiciously selected the MAC monomer as our crosslinking moiety since we did not want to dramatically alter the superior attributes of mPEG- $b$-P(CB-co-LA) copolymer (e.g., drug loading, thermodynamic stability, micelle architecture and biodegradability). MAC has close structural similarity to our well-studied cyclic carbonate (CB) monomer except it has an allyl pendant group in contrast to the phenyl pendant of $\mathrm{CB}$. Therefore, we were confident that the introduction of MAC will not significantly change the backbone or hydrophilic lipophilic balance (HLB) of the polymer. We synthesized MAC monomer using two approaches (Figure 6-1) and used ${ }^{1} \mathrm{H}$ NMR and FT-IR spectrometry to confirm its structure. No FTIR data was found in the literature, however, our ${ }^{1} \mathrm{H}$ NMR spectrum matched the literature [274]. While the first approach had a shorter overall reaction time, the second approach was preferred since it resulted in higher MAC yields (70\% vs. 30\%) and had fewer steps (2 vs. 4). Our MAC yield of $70 \%$ was comparable to that reported by Hu and coworkers [274]. Furthermore, unlike the first approach, which required column chromatography, pure compounds could be obtained with recrystallization in the second approach, making it less labor intensive. Recently, Chen et al. also synthesized a couple of cyclic carbonate monomers similar to MAC [282]. However, their synthetic scheme required four steps, with overall yields of $40 \%$, which is lower than our preferred second approach.

mPEG- $b$-P(CB-co-LA) copolymers previously synthesized using $\mathrm{Sn}(\mathrm{Oct})_{2}$ catalyzed ring-opening polymerization (ROP) exhibited relatively high PDIs (around 1.4) possibly due to undesired transesterification [170]. Furthermore, increasing CB content affected lactide reactivity. Therefore, we used the organic base catalyst DBU ( $\mathrm{pKa}=$ 24.3)[283] for synthesizing $\mathrm{mPEG}_{114}-b-\mathrm{PMAC}_{2.5}-b-\mathrm{P}\left(\mathrm{CB}_{9}-c o-\mathrm{LA}_{39}\right)$ and $\mathrm{mPEG}_{114}-b-$ $\mathrm{P}\left(\mathrm{CB}_{8}-\mathrm{co}-\mathrm{LA}_{35}-\mathrm{co}-\mathrm{MAC}_{2.5}\right)$ copolymers. $\mathrm{DBU}$ is known to mitigate unwanted transesterification resulting in polymers with well controlled molecular weights and low PDIs. In synthesizing $\mathrm{mPEG}_{114}-b$ - $\mathrm{PMAC}_{2.5}-b-\mathrm{P}\left(\mathrm{CB}_{9}-c o-\mathrm{LA}_{39}\right)$ we first sought to understand how the reactivity of MAC compares with $\mathrm{CB}$ using $\mathrm{mPEG}$ as a macro initiator. Our findings suggest the reactivity of MAC ( $80 \%$ conversion in $2 \mathrm{~h}$ (Figure 6-4) to be similar to that of $\mathrm{CB}$ (data not shown) indicating the pendant group does not significantly impact the reactivity of the cyclic carbonate monomers. The DBU catalyzed conversion of our cyclic carbonates is higher than that of trimethyl carbonate (TMC) ( $80 \%$ vs $70 \%)$ reported by Watanabe and coworkers; however, they used 4(Chloromethyl)benzyl alcohol as an initiator [284]. Our kinetic study suggests MAC polymerization with $\mathrm{mPEG}$ to be first-order as demonstrated by the linear nature of the $\ln (1 /(1-\mathrm{x}))$ vs. time plot (Figure 6-4B). This result is not surprising since DBU and $\mathrm{mPEG}$ concentrations do not change during polymerization. Qian et al. have reported similar first-order dependency with respect to monomer, DBU and macro initiator concentrations for ring opening polymerization (ROP) of lactide and glycolide [285]. 
Furthermore, ROP of valerolactone using similar amidine catalysts have been shown to follow first-order kinetics with respect to catalyst, alcohol initiator and monomer concentration [286]. Although we did not systematically study the polymerization kinetics upon addition of $\mathrm{CB}$ and LA, a time of $3 \mathrm{~h}$ was sufficient to obtain polymers with targeted mass ratios of $\mathrm{MAC}, \mathrm{CB}$ and $\mathrm{LA}$ for both $\mathrm{mPEG}_{114}-b-\mathrm{PMAC}_{2.5}-b-\mathrm{P}\left(\mathrm{CB}_{9}-\mathrm{co}-\right.$ $\left.\mathrm{LA}_{39}\right)$ and $\mathrm{mPEG}_{114}-b-\mathrm{P}\left(\mathrm{CB}_{8}-c o-\mathrm{LA}_{35}-c o-\mathrm{MAC}_{2.5}\right)$ copolymers (inferred from ${ }^{1} \mathrm{H} \mathrm{NMR}$ spectra). Furthermore, DBU catalyzed polymerization allowed us to obtain polymers in a relatively short time and avoided MAC crosslinking observed during initial polymerization studies attempted using $\mathrm{Sn}(\mathrm{Oct})_{2}$ at $120^{\circ} \mathrm{C}$.

Monomer arrangement in a copolymer significantly impacts its physico-chemical properties and consequently key micelle properties. Consequently, we performed microstructure analysis on $\mathrm{mPEG}_{114}-b$ - $\mathrm{PMAC}_{2.5}-b-\mathrm{P}\left(\mathrm{CB}_{9}-c o-\mathrm{LA}_{39}\right)$ and $\mathrm{mPEG}_{114}-b$ $\mathrm{P}\left(\mathrm{CB}_{8}-\mathrm{co}-\mathrm{LA}_{35}-\mathrm{co}-\mathrm{MAC}_{2.5}\right)$ copolymers to confirm MAC arrangement in the copolymers. By systematically observing the shift in $65.82 \mathrm{ppm}$ (secondary carbon) and $46.48 \mathrm{ppm}$ (quaternary carbon) peaks in the ${ }^{13} \mathrm{C}$ NMR spectra of mPEG- $b$-PMAC, mPEG- $b$-PMAC- $b$-P(CB- $c o-\mathrm{LA})$, mPEG- $b$-P(CB- $c o-\mathrm{LA}-c o-\mathrm{MAC})$ and mPEG- $b$-P(CB$c o$-LA) copolymers, we were able to show MAC arrangement to be block in mPEG- $b$ PMAC- $b$-P(CB-co-LA) and random in mPEG- $b$-P(CB-co-LA-co-MAC). Typically, reported microstructure analyses of ester based copolymers utilize the carbonyl group in the polymer main chain. However, the close similarity between the contribution of MAC and $\mathrm{CB}$ to copolymer backbone complicates such analysis and it is not feasible to use carbonyl groups in the polymer backbone for microstructure analysis in the current study.

Although inclusion of MAC monomer added a layer of complexity in terms of microstructure analysis, our results suggest that it does not detrimentally affect micelle forming ability or key properties. Both $\mathrm{mPEG}_{114}-b-\mathrm{PMAC}_{2.5}-b-\mathrm{P}\left(\mathrm{CB}_{9}-c o-\mathrm{LA}_{39}\right)$ and $\mathrm{mPEG}_{114}-b-\mathrm{P}\left(\mathrm{CB}_{8}-c o-\mathrm{LA}_{35}-c o-\mathrm{MAC}_{2.5}\right)$ micelles exhibited similar size (with or without drug) which was slightly larger than $\mathrm{mPEG}-b-\mathrm{P}(\mathrm{CB}-c o-\mathrm{LA})$ micelles prepared in our previous study [170]. Since micelle size is dependent on core composition and length (molecular weight), it appears this increase may be due to the contribution of the MAC monomer and relatively larger quantities of lactide present in $\mathrm{mPEG}_{114}-b-\mathrm{PMAC}_{2.5}-b$ $\mathrm{P}\left(\mathrm{CB}_{9}-c o-\mathrm{LA}_{39}\right)$ and $\mathrm{mPEG}_{114}-b-\mathrm{P}\left(\mathrm{CB}_{8}-c o-\mathrm{LA}_{35}-c o-\mathrm{MAC}_{2.5}\right)$ copolymers compared to $\mathrm{mPEG}_{114}-b-\mathrm{P}\left(\mathrm{CB}_{8}-c o-\mathrm{LA}_{24}\right)$ and $\mathrm{mPEG}_{114}-b-\mathrm{P}\left(\mathrm{CB}_{9}-c o-\mathrm{LA}_{5}\right)$ copolymers. Our results are consistent with the fact that hydrophobic blocks with higher molecular weight have generally been shown to have larger sizes. Notwithstanding, drug loading and encapsulation efficiency in crosslinked micelles were comparable to $\mathrm{mPEG}_{114}-b$ - $\mathrm{P}\left(\mathrm{CB}_{8}-\right.$ $\left.c o-\mathrm{LA}_{24}\right)$ and $\mathrm{mPEG}_{114}-b-\mathrm{P}\left(\mathrm{CB}_{9}-c o-\mathrm{LA}_{5}\right)$ micelles possessing similar $\mathrm{CB}$ content. The difference in bicalutamide loading between $\mathrm{mPEG}_{114}-b-\mathrm{PMAC}_{2.5}-b-\mathrm{P}\left(\mathrm{CB}_{9}-c o-\mathrm{LA}_{39}\right)$ and $\mathrm{mPEG}_{114}-b-\mathrm{P}\left(\mathrm{CB}_{8}-c o-\mathrm{LA}_{35}-c o-\mathrm{MAC}_{2.5}\right)$ micelles may be attributed to $\mathrm{CB}$ content. Our previous studies clearly showed that $\mathrm{CB}$ degree of polymerization (DP) of nine resulted in a precipitous decrease in bicalutamide loading compared to DP of 8 [170]. Furthermore, our results provide indirect evidence supporting our hypothesis that the structure and quantities of MAC present in the copolymer do not significantly alter drug loading characteristics. 
Interestingly, $\mathrm{mPEG}_{114}-b-\mathrm{PMAC}_{2.5}-b-\mathrm{P}\left(\mathrm{CB}_{9}-c o-\mathrm{LA}_{39}\right)$ and $\mathrm{mPEG}_{114}-b-\mathrm{P}\left(\mathrm{CB}_{8}-c o-\right.$ $\left.\mathrm{LA}_{35}-c o-\mathrm{MAC}_{2.5}\right)$ micelles exhibited CMCs better than or comparable to mPEG- $b-\mathrm{P}(\mathrm{CB}-$ co-LA) micelles with similar $\mathrm{CB}$ content suggesting equivalent if not better thermodynamic stability. Specifically, $\mathrm{mPEG}_{114}-b-\mathrm{PMAC}_{2.5}-b-\mathrm{P}\left(\mathrm{CB}_{9}-c o-\mathrm{LA}_{39}\right)$ micelles had a $\mathrm{CMC}$ of $0.0008 \mathrm{~g} / \mathrm{L}$ while previously reported $\mathrm{CMC}$ of $\mathrm{mPEG}_{114}-b-\mathrm{P}\left(\mathrm{CB}_{9}-c o-\mathrm{LA}_{5}\right)$ micelles was $0.004 \mathrm{~g} / \mathrm{L}$. Since CB content is the same in both copolymers, this five-fold improvement in CMC value is due to the presence of MAC and LA. However, CMC depends on both composition and molecular mass of the hydrophobic core [287, 288]. Therefore, it is difficult to clearly ascertain the individual contribution of MAC due to considerable difference in overall core length between $\mathrm{mPEG}_{114}-b-\mathrm{PMAC}_{2.5}-b-\mathrm{P}\left(\mathrm{CB}_{9}-c o-\right.$ $\left.\mathrm{LA}_{39}\right)\left(\mathrm{M}_{\mathrm{n}}=9992 \mathrm{~g} / \mathrm{mol}\right)$ and $\mathrm{mPEG}_{114}-b-\mathrm{P}\left(\mathrm{CB}_{9}-c o-\mathrm{LA}_{5}\right)\left(\mathrm{M}_{\mathrm{n}}=7510 \mathrm{~g} / \mathrm{mol}\right)$. Additionally, $\mathrm{mPEG}_{114}-b-\mathrm{P}\left(\mathrm{CB}_{8}-c o-\mathrm{LA}_{35}-c o-\mathrm{MAC}_{2.5}\right)$ micelles had a $\mathrm{CMC}$ of $0.001 \mathrm{~g} / \mathrm{L}$ compared to $0.002 \mathrm{~g} / \mathrm{L}$ for $\mathrm{mPEG}_{114}-b-\mathrm{P}\left(\mathrm{CB}_{8}-c o-\mathrm{LA}_{24}\right)$ micelles reported previously. Here, the modest improvement is most likely due to the presence of MAC since $\mathrm{CB}$ content is the same and the LA amounts are relatively close. Our results demonstrate that the $\mathrm{CMC}$ of $\mathrm{mPEG}_{114}-b-\mathrm{PMAC}_{2.5}-b-\mathrm{P}\left(\mathrm{CB}_{9}-c o-\mathrm{LA}_{39}\right)$ and $\mathrm{mPEG}_{114}-b-\mathrm{P}_{\left(\mathrm{CB}_{8}-c o-\mathrm{LA}_{35}-c o-\right.}$ $\mathrm{MAC}_{2.5}$ ) micelles is sensitive to the presence of MAC but more importantly to its location and arrangement: block versus random distribution among $\mathrm{CB}$ and LA.

Crosslinking based on double bonds requires a radical initiator and exposure to heat or ultraviolet (UV) light. Our copolymers were successfully crosslinked using AIBN as radical initiator at a temperature of $70^{\circ} \mathrm{C}$ for $24 \mathrm{~h}$. We observed crosslinking efficiency of approximately $70 \%$ which was slightly lower than the $80 \%$ value reported in the literature [277]. Complete crosslinking may not be possible since all the allyl moieties present will have to be in close proximity for this to occur. However, it is unlikely that micelle architecture would permit all the double bonds to be within the effective distance required for crosslinking. Theoretically, a few rightly positioned crosslinks would be sufficient to improve micelle mechanical integrity and $100 \%$ crosslinking efficiency is not required. We found crosslinking of blank micelles caused a modest increase in size. This we believe may be an artifact of the crosslinking process since THF used in the process is known to cause micelles to swell. Unexpectedly, crosslinking of drug loaded micelles resulted in a decrease in micelle size regardless of MAC location. This was accompanied by a small loss in drug loading and encapsulation efficiency post crosslinking. The reduction in drug loading and encapsulation efficiency may be due to leakage during the crosslinking process and may account for the modest size decrease observed after crosslinking.

Polymeric micelles are dynamic in nature and gradually disintegrate under sub CMC conditions. Therefore, monitoring changes in micelle size (using DLS) at different dilutions is an elegant way to determine micelle stability. Our results reveal crosslinked micelles remain intact even below the CMC (Figure 6-13A) while non-crosslinked micelles disintegrated. CMC for crosslinked micelles was found to be 20 -fold lower compared to non-crosslinked micelles confirming the fact that crosslinked micelles were several times more stable than their non-crosslinked counterparts. It is worth mentioning that, although $\mathrm{CMC}$ of crosslinked micelles has little practical meaning it still provides a reasonable measure of the extent in improvement in micelle mechanical integrity. 
Micelles have also been shown to become unstable once they encounter blood components [266, 287, 289]. Instability may result from protein adsorption, protein penetration or drug extraction [287]. Since the most abundant protein in blood plasma is serum albumin, we investigated the effect of physiological simulating concentrations of BSA $(45 \mathrm{mg} / \mathrm{mL})$ on crosslinked and non-crosslinked micelle stability using DLS to observe time dependent changes in micelle size. Our results showed significant increase in size of non-crosslinked micelles with time and reflect aggregation of micelles in BSA. This phenomenon may be due to protein adsorption which is undesirable since it can result in fast clearance by the mononuclear phagocyte system (MPS) [290]. It has been shown that BSA-micelle interaction is typically driven by hydrophobic aggregation. For instance, micelles with high density hydrophilic corona experience greater steric stabilization and encounter less BSA interaction [291]. Other instances of polymeric micelle aggregation and interaction with BSA have been reported in the literature [291, 292]. In contrast, there was no appreciable increase in the size of crosslinked micelles following incubation with BSA. One reason may be the less dynamic nature of crosslinked micelles which limits the continuous interchange of unimers thereby reducing the possibility of BSA interacting with the hydrophobic core. It is also likely that the presence of covalent crosslinks make it difficult for BSA to disrupt micelle architecture thus preventing BSA-micelle aggregates.

\section{Conclusions}

To address the current need for biodegradable, biocompatible and stable polymeric micelle delivery systems, we report on the synthesis, characterization and evaluation of lactic-acid and carbonate-based copolymers containing an allyl moiety for preparing interface and core crosslinked micelles. A series of complimentary spectroscopic techniques was used to confirm successful synthesis by ring opening polymerization, elucidate polymer microstructure and confirm crosslinking. $\mathrm{mPEG}_{114}-b$ $\mathrm{PMAC}_{2.5}-b-\mathrm{P}\left(\mathrm{CB}_{9}-c o-\mathrm{LA}_{39}\right)$ and $\mathrm{mPEG}_{114}-b-\mathrm{P}\left(\mathrm{CB}_{8}-c o-\mathrm{LA}_{35}-c o-\mathrm{MAC}_{2.5}\right)$ copolymers for interface and core crosslinking, respectively, displayed low polydispersity (1.08) and $\mathrm{CMC}$ values $(0.8-1 \mathrm{mg} / \mathrm{L})$. Inclusion of MAC monomer did not significantly alter micelle size or morphology irrespective of polymer composition or MAC location. Importantly, $\mathrm{mPEG}_{114}-b-\mathrm{P}\left(\mathrm{CB}_{8}-c o-\mathrm{LA}_{35}-c o-\mathrm{MAC}_{2.5}\right)$ had a higher drug encapsulation efficiency $(78.72 \% \pm 0.15)$ compared to $\mathrm{mPEG}_{114}-b-\mathrm{PMAC}_{2.5}-b-\mathrm{P}\left(\mathrm{CB}_{9}-c o-\mathrm{LA}_{39}\right)(20.29 \% \pm 0.11)$. Subsequent micelle crosslinking did not dramatically change drug loading or micelle morphology. However, there was strong link between micelle crosslinking and improved stability when incubated in aqueous media under extensive dilution and physiological simulating serum (BSA $\sim 45 \mathrm{mg} / \mathrm{mL}$ ). Crosslinked micelle size remained unchanged while non-crosslinked micelles disintegrated at a thousand fold dilution. Besides, crosslinked micelle size remained unchanged in BSA whereas there was a timedependent increase in the size of non-crosslinked micelles. These results showed crosslinked micelles to be more stable compared to their non-crosslinked counterparts. Additionally, bicalutamide-loaded crosslinked micelles were found to be more potent in inhibiting proliferation of $\mathrm{LNCaP}$ prostate cancer cells compared to non-crosslinked micelles regardless of polymer type. In all, we have demonstrated that these new 
biodegradable copolymer systems are potentially useful for cancer therapy. Future studies on influence of MAC block length, extent of crosslinking efficiency and ratio of MAC to $\mathrm{CB}$ and LA on key micelle properties are required to generate material design rules which can be utilized for customized fabrication of improved micelle delivery platforms. 


\section{CHAPTER 7. ANDROGEN RECEPTOR AND XIAP GENE SILENCING INHIBITS CELL PROLIFERATION AND INDUCES APOPTOSIS IN HORMONE REFRACTORY PROSTATE CANCER}

\section{Introduction}

To date, therapeutic options for advanced prostate cancer are typically palliative and largely ineffective in the long term. Profound gaps in our understanding regarding mechanisms leading to progression of prostate cancer coupled with multidrug resistance (MDR) associated with small molecule based therapies and lack of enabling delivery technologies has hindered the emergence of potent therapies against advanced prostate cancer. Presently, the key role of the androgen receptor (AR) in prostate cancer progression has widely been accepted. For instance, it has been reported that all prostate cancers express the AR regardless of stage [293]. However, AR undergoes some characteristic mechanistic changes during progression to its advanced stage which render present anti-androgens (e.g., bicalutamide) ineffective. First, there is an altering of ligand specificity due to mutations in the AR leading to aberrant activation by non-androgenic molecules including anti-androgens [294]. Secondly, approximately a third of prostate cancers that recur due to failing endocrine therapy contain an AR gene amplification $[295,296]$. In such cases, AR is overexpressed and causes prostate cancer cells to be hypersensitive to low levels of androgens resulting in continual androgen-dependent growth [296, 297]. These evidences suggest that AR signaling indeed plays a pivotal role in development and progression of prostate cancer. Therefore, emerging therapeutic strategies should include effectual approaches of down-regulating AR gene expression in cancerous cells rather than mere inactivation.

Resistance to apoptosis is a hallmark of progression to advanced prostate cancer. This acquired defect in the apoptotic machinery arises from incapacitating mutations which provides cancer cells with an undesirable proliferation edge [298, 299].

Consequently, regulators of apoptosis such as Bcl-2 and inhibitors of apoptosis protein (IAPs) have attracted attention as potential therapeutic targets. IAPs prevent cell death in response to external stimuli by inhibiting downstream portions of the apoptotic pathway through endogenous suppression of caspase activity [182-184]. Among the various IAPs, $\mathrm{X}$ chromosome-linked IAP (XIAP) is the most potent and thoroughly characterized [184]. XIAP protein binds and inhibits the initiator caspase- 9 and effector caspase- 3 and caspase-7 through the binding of its BIR3 domain and the linker region between BIR1 and BIR2, respectively; consequently inhibiting both intrinsic and extrinsic apoptotic pathways [185-188, 300]. The importance of XIAP as a therapeutic target has been supported by several lines of evidence. For example, expression levels of XIAP in human prostate cancer cells were found to correlate with apoptotic resistance [189, 190]. Also, in vitro studies showed XIAP overexpression to confer multiagent chemoresistance. Consistent with this hypothesis, down-regulation of XIAP using RNA interference (RNAi) technology and antisense oligonucleotides was found to sensitize malignant cell lines to chemotherapy [301-304]. 
We have previously shown that combination therapy targeting AR and XIAP using small molecules, such as bicalutamide and embelin is a promising approach to treat prostate cancer [11]. However, prolonged treatment results in bicalutamide functioning as agonists rather than antagonists due to AR mutations and amplification. Furthermore, most chemotherapeutics including embelin eventually succumb to multidrug resistance (MDR) due to the presence of ATP-Binding Cassette (ABC) transporters in cancer cells. Since RNA interference (RNAi) technology can overcome these major obstacles, we hypothesize that AR and XIAP gene silencing using RNAi technology will be more potent in treating advanced prostate cancer (Figure 7-1).

RNAi is a double-stranded RNA-dependent endogenous gene silencing mechanism resulting in sequence-specific mRNA degradation [305]. Application of RNAi is gaining popularity in oncology as a therapeutic approach since theoretically any oncogene can be silenced. RNAi may be achieved using chemically synthesized small interefering RNA (siRNA) or vector based short hairpin RNA (shRNA). Synthesized siRNAs typically elicit quick gene silencing, however they are costly and the duration of silencing is short. In contrast, vector based shRNA results in longer gene silencing and is relatively cheaper [306]. Despite their potential benefits, clinical translation of siRNA and shRNAs has been limited by their low cellular uptake and susceptibility to enzymatic degradation in vivo due to their large molecular weight and negative charge. Hence, there is an urgent need to develop carriers for siRNA delivery. Polymeric carriers for RNAi have garnered interest since they can be engineered to possess moieties that can effectively bind and condense siRNA, enhance cellular uptake and reduce enzymatic degradation.

In this work, we designed and screened six chemically synthesized siRNAs: three siRNAs targeting distinct regions of AR and XIAP mRNA, respectively. The most potent siRNA sequences for both genes were then selected and their effect on inducing apoptosis and inhibiting cell proliferation in prostate cancer cells as monotherapy or combination therapy examined. Synthetic siRNAs are prone to in vivo instability and tend to be exhorbitant. Therefore, we converted potent siRNA sequences against AR and XIAP into shRNAs and constructed a bipartite plasmid using psiRNA-DUO vector. We demonstrate that our bipartite plasmid effectively silences AR and XIAP gene expression and can lead to longer silencing effect compared to synthetic siRNA. Finally, we also synthesized and characterized a novel biodegradable and non-toxic N-(2-hydroxypropyl) methacrylamide (HPMA) based copolymer composed of polycationic, lipid and pka modulator moieties and report on its potential for efficient delivery of siRNA and shRNA plasmid vectors. 


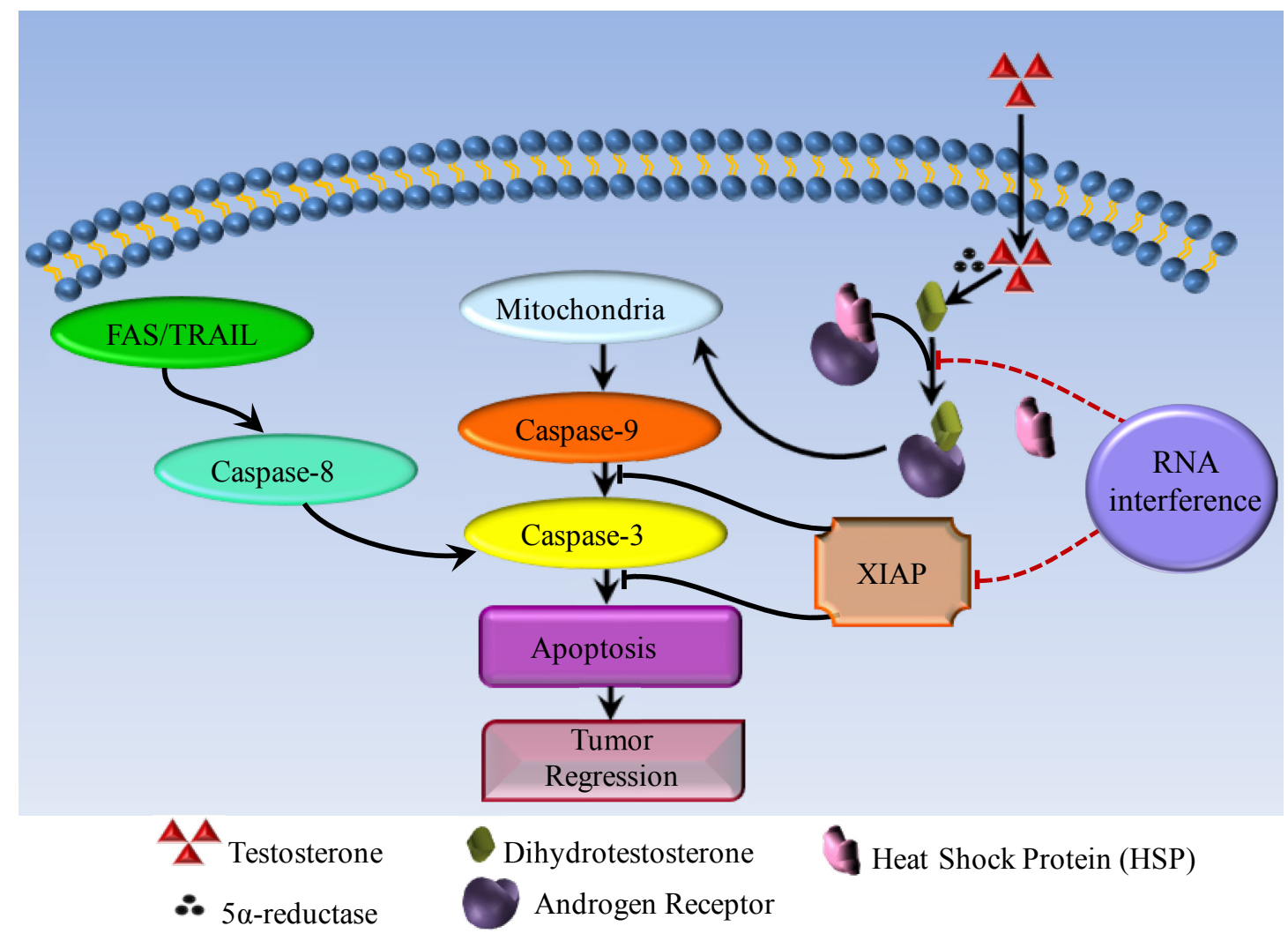

Figure 7-1. Schematic diagram showing the combined effect of androgen receptor and XIAP gene silencing on apoptosis and tumor regression.

Down-regulation of AR results in the inhibition of proliferation and invasion while downregulation of XIAP facilitates apoptotic mechanism. Hence, down-regulation of both AR and XIAP pathways results in potential synergistic effect in regressing tumors. 


\section{Materials and Methods}

Materials

Human prostate cancer cell lines (LNCaP and C4-2) were cultured in Roswell Park Memorial Institute (RPMI) 1640 medium (Invitrogen, Carlsbad, CA), with 10\% fetal bovine serum (FBS) and 1\% Antibiotic-Antimycotic (100 x) (Invitrogen, Carlsbad, $\mathrm{CA}$ ) in a $5 \% \mathrm{CO}_{2}$ atmosphere at $37^{\circ} \mathrm{C}$. TrypLE ${ }^{\mathrm{TM}}$ Express and Lipofectamine 2000 were procured from Invitrogen (Carlsbad, CA). Bovine serum albumin (BSA) (purity $>96 \%$ ) was obtained from Sigma-Aldrich (St. Louis, MO). SYBR Green real time PCR master mix and reverse transcription reagents were purchased from Applied Biosystems (Foster city, CA). Human Total XIAP ELISA kit was purchased from R\&D Systems (Minneapolis, MN). Primary and secondary antibodies were purchased from Abcam (Cambridge, MA). All other reagents were obtained from Sigma-Aldrich (St. Louis, MO) unless otherwise stated and were used as received.

Design and synthesis of siRNA

Three synthetic siRNAs targeting androgen receptor (AR) mRNA (Accension no.: NM_000044) and XIAP mRNA (Accension no.: NM_001167) were designed using BLOCK-iT ${ }^{\mathrm{TM}}$ RNAi Designer and obtained from Invitrogen (Carlsbad, CA). siRNA sequences and their start sites are listed in Table 7-1. The designed siRNA sequences were blasted against the human genome database to eliminate cross-silencing of nontarget genes.

Synthesis of HPMA-g-APMA-g-MM-g-HM copolymers

Copolymers were synthesized by free radical copolymerization of N-(2hydroxypropyl)methacrylamide (HPMA), N-(3-aminopropyl)methacrylamide hydrochloride (APMA), morpholinoethyl methacrylate (MM), and n-hexyl methacrylate (HM) in DMSO at $60{ }^{\circ} \mathrm{C}$ for $24 \mathrm{~h}$ using 2,2'-azobisisobutyronitrile (AIBN) as an initiator and mercaptopropanol as a chain transfer agent. Typically, HPMA (200 mg, $1.4 \mathrm{mmol}$ ), APMA (21 mg, $0.12 \mathrm{mmol}), \mathrm{MM}$ (46.3 mg, $0.23 \mathrm{mmol}$ ), and HM (138 mg, $0.70 \mathrm{mmol})$, AIBN (15 mg, $3 \mathrm{~mol} \%$ ) and Mercaptopropanol (12 $\mu \mathrm{L}, 6 \mathrm{~mol} \%)$ were dissolved in DMSO (2.45 ml, $15 \mathrm{wt} \%)$, placed in an ampoule, purged with nitrogen for $5 \mathrm{~min}$, and sealed. After the reaction the polymer was dissolved in water and dialyzed for $48 \mathrm{~h}$ using a 5000 MWCO membrane to remove unreacted monomer. The polymers were isolated by freeze drying with a yield of $70 \%$ and characterized using ${ }^{1} \mathrm{H}-\mathrm{NMR}$ using d-Methanol as solvent (Table 7-2). 
Table 7-1. Small interfering RNA (siRNA) against human androgen receptor and X-linked inhibitor of apoptosis.

\begin{tabular}{llc}
\hline Gene & Sequence & Start site \\
\hline AR & (NM_000044) & 1172 \\
& CCGAGGAGCUUUCCAGAAU & 1648 \\
& CCGCUGACCUUAAAGACAU & 1768 \\
& CCACUUCCUCCAAGGACAA & \\
XIAP & (NM_001167) & 264 \\
& ACACUGGCAGAGCAGGGUUUCUUU & 297 \\
& GAAGGAGAUACCGUGCGGUGCUUA & 458 \\
& CCAGAAUGGUCAGUACAAAGUUGAA & \\
\hline
\end{tabular}


Table 7-2. Characterization of HPMA-g-APMA-g-MM-g-HM copolymers.

\begin{tabular}{|c|c|c|c|c|c|c|c|c|c|c|}
\hline Polymer & HPMA $^{\mathrm{a}}$ & HPMA $^{b}$ & $\mathbf{H M}^{\mathbf{a}}$ & $\mathbf{H M}^{\mathbf{b}}$ & $\mathbf{M M}^{\mathbf{a}}$ & $\mathbf{M M}^{\mathbf{b}}$ & APMA $^{a}$ & $\mathbf{A P M A}^{\mathbf{b}}$ & $\begin{array}{l}\mathrm{MW}^{\mathbf{c}} \\
(\mathbf{k D a})\end{array}$ & PDI $^{\mathrm{d}}$ \\
\hline $\begin{array}{l}\mathrm{HPMA}_{16}-\mathrm{g}-\mathrm{APMA}_{1}-\mathrm{g}- \\
\mathrm{MM}_{33}-\mathrm{g}-\mathrm{HM}_{50}\end{array}$ & 18.2 & 15.7 & 41.8 & 49.5 & 30 & 33.4 & 10 & 1.4 & 8.7 & 1.3 \\
\hline $\begin{array}{l}\mathrm{HPMA}_{32}-\mathrm{g}-\mathrm{APMA}_{3}-\mathrm{g}- \\
\mathrm{MM}_{22}-\mathrm{g}-\mathrm{HM}_{43}\end{array}$ & 26.6 & 32.1 & 33.4 & 42.9 & 20 & 22.3 & 20 & 2.7 & 8.3 & 1.2 \\
\hline
\end{tabular}


Construction of shRNA expression plasmids

Two potent siRNAs targeting AR (start site 3008) and XIAP (start site 297) were converted into shRNA (Table 7-3) and utilized for plasmid construction. pshRNA-3008, pshRNA-297 and pshRNA-3008-297 vectors were constructed using psiRNA-DUO vector, which contains two shRNA expression cassettes controlled by a human 7SK RNA polymerase III promoter and contains a green fluorescence protein (GFP) reported gene (InvivoGen, San Diego, CA). shRNAs were obtained by annealing two complementary oligonucleotides and ligated into psiRNA-DUO vector at $27^{\circ} \mathrm{C}$ for $2 \mathrm{~h}$ using T4 DNA ligase. Subsequently, the ligation mixture was transformed into XL2-blue ultracompetent cells (Agilent Technologies Inc, Santa Clara, CA), positive clones identified using white/blue selection, amplified in LB media and plasmids purified using Promega PureYield ${ }^{\mathrm{TM}}$ Plasmid Miniprep Kit (Promega, Madison, WI). Four positive clones were sequenced to verify the shRNA insert sequence.

\section{Transfection of siRNA and shRNA}

LNCaP and C4-2 cells were seeded in 24-well plates at a density of $4 \times 10^{4}$ cells/well and maintained in $0.5 \mathrm{~mL}$ of RPMI 1640 medium containing 10\% fetal bovine serum (FBS) at $37^{\circ} \mathrm{C}$ and $5 \%$ overnight for attachment. siRNA duplexes (40 and $100 \mathrm{nM}$ ) and plasmid $(1 \mu \mathrm{g})$ were complexed with $\mathrm{HPMA}_{16}-\mathrm{g}-\mathrm{APMA}_{1}-\mathrm{g}-\mathrm{MM}_{33}-\mathrm{g}-\mathrm{HM}_{50}$ and HPMA $_{32}$-g-APMA - -g- $\mathrm{MM}_{22}-\mathrm{g}-\mathrm{HM}_{43}$ at a charge ratio of 4:1 and 8:1 (polymer:siRNA). The formulated polyplexes were added and incubated with cells for 48 or $72 \mathrm{~h}$ at $37^{\circ} \mathrm{C}$. siRNA and shRNA effectiveness was assayed using real time RT-PCR, ELISA and Western blot.

\section{Real-time reverse transcription-PCR}

Total RNA was isolated using RNeasy Mini kit (Qiagen, Valencia, CA) and $1 \mu \mathrm{g}$ from each sample was converted to cDNA using MultiScribe Reverse Transcriptase Reagent and random hexamers (Applied Biosystems Inc., Branchburg, NJ). One hundred nanograms of the cDNA was amplified by real time PCR using SYBR Green-1 dye universal master mix on ABI Prism 7700 Sequence Detection System (Applied Biosystems, Inc., Foster City, CA). The primers used were as follows: AR: forward, 5'AGC CAT TGA GCC AGG TGT AG-3'; reverse, 5'-CGT GTA AGT TGC GGA AGC C-3'. PSA: forward, 5'-GTG GGT CCC GGT TGT CT-3'; reverse, 5'-

AGCCCAGCTCCCTGTCT-3'.XIAP: forward, 5'-TGT TTC AGC ATC AAC ACT GGC ACG-3'; reverse, 5'- GCA TGA CAA CTA AAG CAC CGG AC-3'. PCR products were subjected to melting-curve analysis to confirm PCR specificity. The comparative threshold $(\mathrm{Ct})$ method was used for data analysis and normalized with $\beta$-actin. 
Table 7-3. shRNA sequences targeting specific regions of androgen receptor (AR) and XIAP genes.

\begin{tabular}{|c|c|}
\hline Vector & shRNA sequence \\
\hline pshRNA-1172 & $\begin{array}{l}\text { Sense: 5' } \\
\text { ACCTCCCGAGGAGCTTTCCAGAATTCAAGAGATTCTGGAAAGCTCCTCGGTT 3“ } \\
\text { Antisense: 5' } \\
\text { CAAAAACCGAGGAGCTTTCCAGAATCTCTTGAATTCTGGAAAGCTCCTCGGG 3' }\end{array}$ \\
\hline pshRNA-1648 & $\begin{array}{l}\text { Sense: 5' } \\
\text { ACCTCCCGCTGACCTTAAAGACATTCAAGAGATGTCTTTAAGGTCAGCGGTT 3“ } \\
\text { Antisense: 5' } \\
\text { CAAAAACCGCTGACCTTAAAGACATCTCTTGAATGTCTTTAAGGTCAGCGGG 3' }\end{array}$ \\
\hline pshRNA-3008 & $\begin{array}{l}\text { Sense: 5' } \\
\text { ACCTCGCTGAAGAAACTTGGTAATTCAAGAGATTACCAAGTTTCTTCAGCTT 3“} \\
\text { Antisense: 5' } \\
\text { CAAAAAGCTGAAGAAACTTGGTAATCTCTTGAATTACCAAGTTTCTTCAGCG 3' }\end{array}$ \\
\hline pshRNA-297 & $\begin{array}{l}\text { Sense: 5' } \\
\text { GTACCTCGAAGGAGATACCGTGCGGTGCTTATCAAGAGTAAGCACCGCACGGT } \\
\text { ATCTCCTTCTTTTTGGAAA 3“' } \\
\text { Antisense: 5' } \\
\text { AGCTTTTCCAAAAAGAAGGAGATACCGTGCGGTGCTTACTCTTGATAAGCACC } \\
\text { GCACGGTATCTCCTTCGAG 3' }\end{array}$ \\
\hline
\end{tabular}

Blue color signifies restriction site. Red color indicates loop sequence. 
Western blot analysis

Cells were transfected with $40 \mathrm{nM}$ of 1172, 1648 and 1768 (Table 7-1) for $72 \mathrm{~h}$. Post treatement, cells were lysed using RIPA buffer (Sigma-Aldrich, St. Louis, MO) and protein concentration measured with bicinchoninic acid (BCA) protein assay kit (Pierece, Rockford, IL). The lysate was boiled for 5 mins and subjected to a 15\% SDS-PAGE and transferred to a PVDF membrane using BBlot $^{\mathrm{TM}}$ system (Invitrogen, Carlsbad, CA). The membranes were then blocked with $3 \%$ bovine serum albumin in $1 \mathrm{x}$ tris buffered saline tween (TBST) at room temperature for an hour. Membranes were blocked with 3\% BSA in $1 \mathrm{x}$ tris buffered saline Tween (TBST) at room temperature for $1 \mathrm{~h}$ and then incubated with primary antibodies at $4{ }^{\circ} \mathrm{C}$ overnight, followed by incubation with secondary antibody conjugated with horseradish peroxidase (HRP) at room temperature for $1 \mathrm{~h}$. The signal of target proteins was detected using Immun-Star HRP chemiluminescent kit (BioRad, Hercules, CA).

XIAP concentration detection using ELISA

Cells were transfected with XIAP siRNA at a dose of $40 \mathrm{nM}$ using Lipofectamine 2000 at 3:1 N/P ratio. Seventy-two hours post transfection, cells were lysed and XIAP concentration detected using Human XIAP ELISA kit as described in the manufacturer's protocol.

siRNA uptake and cellular distribution study by flow cytometry and confocal microscopy

The ability of copolymers to transfect siRNA into C4-2 cells was assessed using FAM-labeled siRNA (160 nM) formulated into polyplexes at an N/P ratio of 8 (copolymers). C4-2 cells were plated in 6 well plates $\left(5 \times 10^{4}\right.$ cells/well) and incubated with polyplex for $3 \mathrm{~h}$. Cells were then washed with 1x cold PBS, trypsinized and FAMlabeled siRNA uptake detected by a Becton-Dickinson FACSort ${ }^{\mathrm{TM}}$ flow cytometer (Franklin Lakes, NJ). The data was analyzed with ModFit ${ }^{\mathrm{TM}}$ software (BectonDickinson).

Intracellular trafficking of FAM-labeled siRNA was also evaluated using confocal fluorescent microscopy. Polyplexes were formulated using copolymer at N/P ratio of 8 . Cells were cultured in 8 well chamber plates and incubated with polyplexes for $3 \mathrm{~h}$. Afterward, cells were washed thrice with 1x PBS, fixed in paraformaldehyde in PBS for 10 min. For nucleus labeling, fixed cells were washed with PBS and then incubated with 4',6-diamidino-2-phenylindole (DAPI) (Molecular Probes, Invitrogen Co., OR, USA) for 15 min. Localization of complexes in cells was visualized by a Zeiss 510 LSMNLO confocal microscope (Carl Zeiss Microscope systems, Jena, Germany) with identical settings for each confocal study. 
In vitro cell viability assays

LNCaP and C4-2 cells (American Type Culture Collection) were incubated in RPMI 1640 media, $10 \%$ fetal bovine serum and $1 \%$ antibiotic-antimycotic at $37^{\circ} \mathrm{C}$ in humidified environment of $5 \% \mathrm{CO}_{2}$ and subcultured every 3-4 days to maintain exponential growth. Cells were seeded in 96-well plates at a density of $1 \times 10^{4}$ viable cells/well and incubated for $48 \mathrm{~h}$. The cells were exposed to siRNA targeting AR and XIAP for $96 \mathrm{~h}$. At the end of treatment, $20 \mu \mathrm{l}$ of MTT $(5 \mathrm{mg} / \mathrm{ml})$ was added to each well and incubated for 3-4 h and analyzed at a test wavelength of $560 \mathrm{~nm}$. Cell viability was expressed as a percentage of the intensity of controls.

Apoptosis and cell cycle analysis

Following treatment with siRNA targeting AR and XIAP for $96 \mathrm{~h}$, cells were trypsinized and fixed in 70\% ice-cold ethanol and washed with PBS. Samples were resuspended in $500 \mu$ of propidium iodide solution containing RNAse A (BD Pharmingen, San Diego, CA) for 15 min at room temperature. Relative DNA content per cell was acquired by measuring DNA fluorescence using flow cytometry and subsequent analysis performed with Modfit program.

In vivo efficacy assessment of siRNA/shRNA polyplex in xenografts

All animal experiments were performed in accordance with $\mathrm{NIH}$ animal use guidelines and the protocol approved by the Animal Care and Use Committee at the University of Tennessee Health Science Center. Xenograft flank tumors were induced in 8 week old male athymic nu/nu mice purchased from The Jackson Laboratory (Bar Harbor, ME) by subcutaneous injection of 3 million C4-2 cells suspended in 1:1 media and matrigel. When tumors reached approximately $50 \mathrm{~mm}^{3}$, mice were randomized into five groups of 5 mice, minimizing weight and tumor size differences. Each group was treated with three consecutive intratumoral injection of blank polyplex, polyplex formulated with AR, XIAP and pshRNA-3008-297 (2 mg/kg). Tumors were then isolated, RNA extracted and gene silencing determined using real-time RT-PCR.

\section{Results}

AR and XIAP gene silencing

To determine the ability of siRNA to specifically silence AR and XIAP gene expression, we designed and selected three siRNAs targeting different regions of each gene (Table 7-1). These siRNAs were screened for potency by transient transfection into LNCaP and C4-2 cells upon complex formation with Lipofectamine ${ }^{\mathrm{TM}} 2000$ (transfection efficiency was $90 \pm 7 \%$ as determined by BLOCK-iT ${ }^{\mathrm{TM}}$ Alexa Fluor ${ }^{\circledR}$ red fluorescent 
oligo). Real time RT-PCR was used to quantitatively determine AR and XIAP silencing at mRNA levels. All siRNAs targeting AR $(1172,1648$ and 1768) used in this study silenced AR mRNA expression in both cells. Furthermore, 1172 was identified as the most potent sequence resulting in approximately $75 \%$ knockdown regardless of cell type. In contrast, 1648 and 1768 decreased $A R$ mRNA levels to $26 \%$ and $44 \%$, respectively, in LNCaP cells compared to 53\% and 67\% in C4-2 cells (Figure 7-2A). Also, the three XIAP siRNAs $(264,297$ and 458) tested down-regulated XIAP mRNA levels in both cells and were generally more potent in C4-2 cells compared to LNCaP cells (Figure 7-2B). XIAP mRNA expression in C4-2 cells was reduced to approximately $20 \%$ irrespective of siRNA sequence used. In contrast, 264 and 297 led to approximately 50\% knockdown while 458 resulted in $32 \%$ knockdown in LNCaP cells.

Western blot analysis revealed 1172,1648 and 1768 to significantly decrease expression levels of AR protein in C4-2 cells (Figure 7-2C). We also determined the effect of AR silencing on expression of prostate specific antigen (PSA) gene which is downstream of AR. We found 1172, 1648 and 1768 to decrease PSA protein expression compared to control. ELISA was used to determine the potency of XIAP siRNAs in decreasing C4-2 XIAP protein expression. From Figure 7-2D, all XIAP siRNA sequences significantly decreased XIAP protein expression ( $\sim$ five fold). Consequently, 1172 and 264 were used for further studies on effects associated with dual knockdown of $\mathrm{AR}$ and XIAP genes.

Synthesis and characterization of HPMA-g-APMA-g-MM-g-HM copolymers

HPMA-g-APMA-g-MM-g-HM copolymers (HPMA 16 -g-APMA 1 -g-MM ${ }_{33}$-g$\mathrm{HM}_{50}$ and $\left.\mathrm{HPMA}_{32}-\mathrm{g}-\mathrm{APMA}_{3}-\mathrm{g}-\mathrm{MM}_{22}-\mathrm{g}-\mathrm{HM}_{43}\right)$ were synthesized from N-(2hydroxypropyl)methacrylamide, $\mathrm{N}$-(3-aminopropyl)methacrylamide hydrochloride, morpholinoethyl methacrylate, and n-hexyl methacrylate by free radical copolymerization. The reaction was performed in DMSO at $60^{\circ} \mathrm{C}$ for $24 \mathrm{~h}$ using AIBN as the initiator and mercaptopropanol as a chain transfer agent (Figure 7-3). Following polymerization and purification, the structure of copolymers was confirmed by ${ }^{1} \mathrm{H}$ NMR and size exclusion chromatography (SEC) using multi angle light scattering (MALS) system on Superose 6 column (Table 7-2). The molecular weights (MW) of both copolymers were calculated from ${ }^{1} \mathrm{H}$ NMR spectra and compared to the theoretical MW based on feed ratio and degree of polymerization (dp). We found the estimated MW to be in close agreement with the theoretical molecular weight. Specifically, the actual MW of $\mathrm{HPMA}_{16}$-g-APMA $-\mathrm{g}-\mathrm{MM}_{33}$-g-HM${ }_{50}$ was computed to be $17,580.75 \mathrm{~g} / \mathrm{mol}$ for a theoretical MW of $17,487.19 \mathrm{~g} / \mathrm{mol}$. Also, the calculated MW of HPMA $32-\mathrm{g}-\mathrm{APMA}_{3}-\mathrm{g}-$ $\mathrm{MM}_{22}-\mathrm{g}-\mathrm{HM}_{43}$ was $16,826.22 \mathrm{~g} / \mathrm{mol}$ while the theoretical MW was $17,054.47 \mathrm{~g} / \mathrm{mol}$. For both copolymers HPMA, HM and MM monomers were essentially $100 \%$ converted in 24 $\mathrm{h}$ using our reaction conditions (ref. Materials and Methods). Interestingly, we found APMA monomer to be only 14\% converted for both copolymers. Further characterization using SEC revealed $\mathrm{HPMA}_{32}$-g-APMA $-\mathrm{g}-\mathrm{MM}_{22}$-g-HM $\mathrm{H}_{43}$ had a molecular weight of $8,300 \mathrm{~g} / \mathrm{mol}$ and polydispersity (PDI) of 1.2 while $\mathrm{HPMA}_{16}-\mathrm{g}-\mathrm{APMA}_{1}-\mathrm{g}-\mathrm{MM}_{33}-\mathrm{g}-\mathrm{HM}_{50}$ had molecular weight of $8,700 \mathrm{~g} / \mathrm{mol}$ and PDI of 1.3 . 

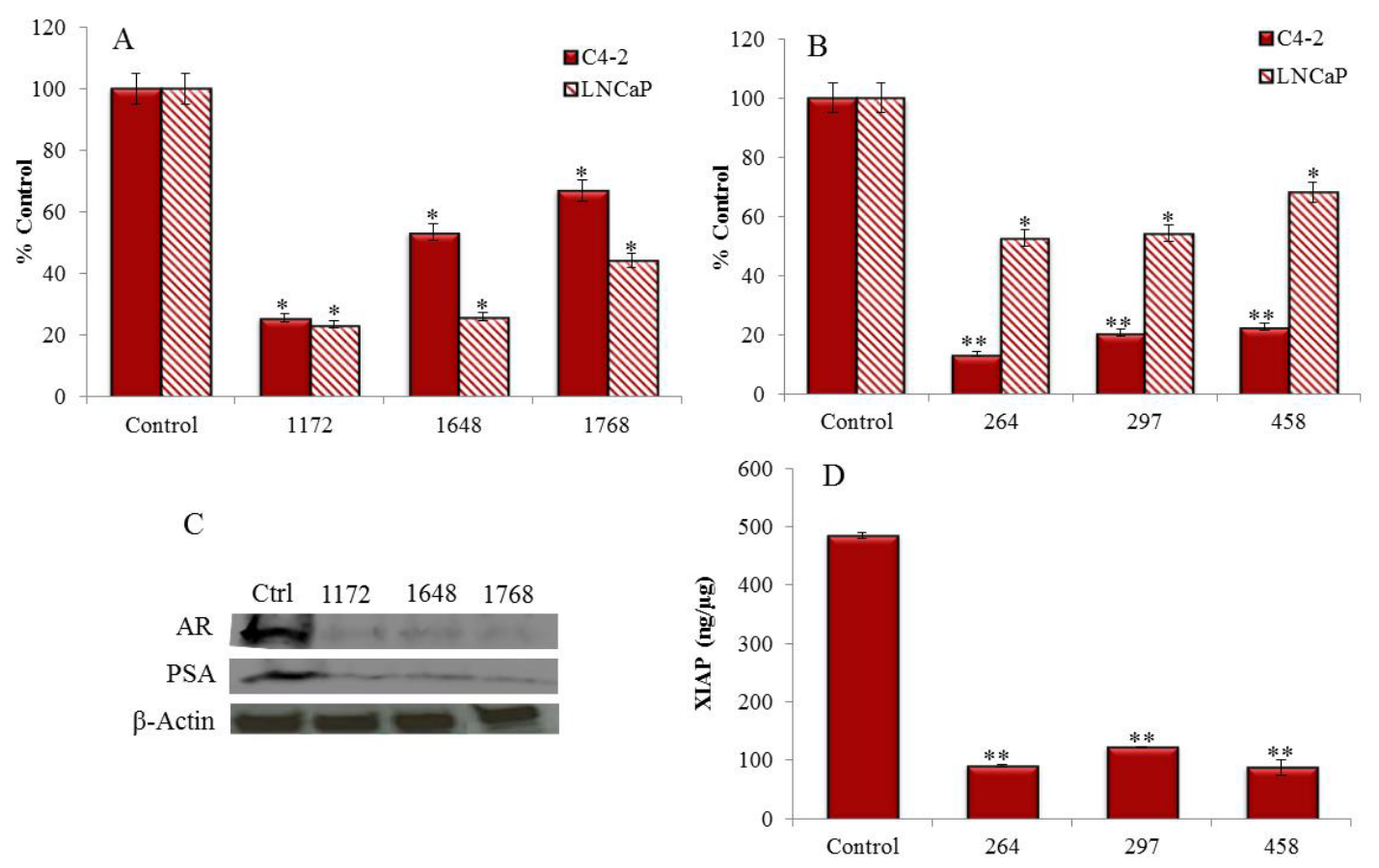

Figure 7-2. Effect of siRNA sequence on AR and XIAP gene silencing after complex formation with Lipofectamine 2000.

Three different siRNAs targeting different regions of AR and XIAP mRNA were transfected into LNCaP and C4-2 cells at a concentration of $40 \mathrm{nM}$ for $48 \mathrm{~h}$. (A) AR gene silencing determined by real time RT-PCR. (B) XIAP gene silencing determined by real time RT-PCR. (c) Western blot analysis of AR and PSA protein expression in C4-2 cells $96 \mathrm{~h}$ post transfection with $40 \mathrm{nM}$ and (D) ELISA analysis of XIAP protein expression in C4-2 cells $96 \mathrm{~h}$ post transfection with $40 \mathrm{nM}$. Results were represented as the mean $\pm \mathrm{SD}$ $(n=3) .{ }^{*} p<0.05,{ }^{* *} p<0.01$ compared to control. 


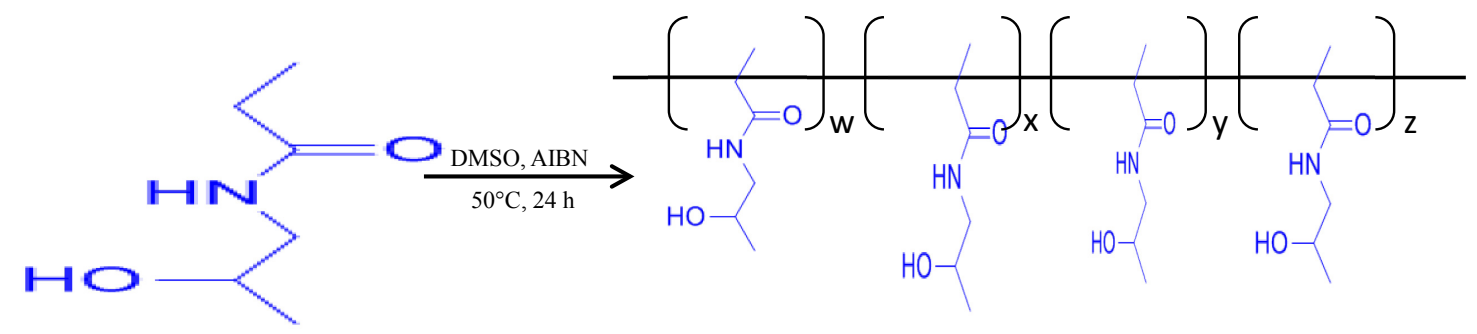

Figure 7-3. Synthesis of HPMA-g-APMA-g-MM-g-HM copolymers. 
Effect of HPMA-g-APMA-g-MM-g-HM copolymers on nucleic acid condensation and polyplex size

Since charge (N/P) ratio affects the electrostatic interaction between polymer and siRNA, we determined the siRNA condensing ability of $\mathrm{HPMA}_{32}-\mathrm{g}-\mathrm{APMA}_{3}-\mathrm{g}-\mathrm{MM}_{22}$-g$\mathrm{HM}_{43}$ and $\mathrm{HPMA}_{16}$-g-APMA - -g-MM $\mathrm{MM}_{33}-\mathrm{g}-\mathrm{HM}_{50}$ copolymers at specified charge (N/P) ratios using the ethidium bromide (EB) fluorescence quenching assay. From Figure 7-4A, both copolymers were able to effectively bind siRNA. However, HPMA 32 -g$\mathrm{APMA}_{3}-\mathrm{g}-\mathrm{MM}_{22}-\mathrm{g}-\mathrm{HM}_{43}$ showed superior fluorescence quenching compared to $\mathrm{HPMA}_{16}-\mathrm{g}-\mathrm{APMA}_{1}-\mathrm{g}-\mathrm{MM}_{33}-\mathrm{g}-\mathrm{HM}_{50}$ at N/P ratio above 4.

We next examined the effect of N/P ratio on the size of $\mathrm{HPMA}_{32}$-g-APMA - -g$\mathrm{MM}_{22}-\mathrm{g}-\mathrm{HM}_{43}$ and $\mathrm{HPMA}_{16}-\mathrm{g}-\mathrm{APMA}_{1}-\mathrm{g}-\mathrm{MM}_{33}-\mathrm{g}-\mathrm{HM}_{50}$ polyplexes. In general, increase in N/P ratio led to a decrease in polyplex size (Figure 7-4B). HPMA 16 -g-APMA - $^{-g}$ $\mathrm{MM}_{33}-\mathrm{g}-\mathrm{HM}_{50}$ polyplex size decreased from $368 \pm 3.24 \mathrm{~nm}(\mathrm{~N} / \mathrm{P}=1)$ to $\sim 270 \mathrm{~nm}(\mathrm{~N} / \mathrm{P}=$ 4 and 8). In contrast, polyplexes formulated using $\mathrm{HPMA}_{32}-\mathrm{g}-\mathrm{APMA}_{3}-\mathrm{g}-\mathrm{MM}_{22}$-g$\mathrm{HM}_{43}$ copolymer decreased from $251 \pm 9.3 \mathrm{~nm}(\mathrm{~N} / \mathrm{P}=1)$ to $\sim 131 \mathrm{~nm}(\mathrm{~N} / \mathrm{P}=4$ and 8$)$ and were in general smaller than $\mathrm{HPMA}_{16}-\mathrm{g}-\mathrm{APMA}_{1}-\mathrm{g}-\mathrm{MM}_{33}-\mathrm{g}-\mathrm{HM}_{50}$ polyplexes at each $\mathrm{N} / \mathrm{P}$ ratio.

Effect of charge ratio on cellular uptake of polymer/siRNA complexes

The effect of charge ratio on cellular uptake of $\mathrm{HPMA}_{32}-\mathrm{g}-\mathrm{APMA}_{3}-\mathrm{g}-\mathrm{MM}_{22}$-g$\mathrm{HM}_{43} /$ siRNA complexes in C4-2 prostate cancer cells was determined using FAMlabeled siRNA and flow cytometry. As shown in Figure 7-4C, increasing N/P ratio led to increase in the uptake of polyplexes in C4-2 cells. Specifically, cellular uptake of $\mathrm{HPMA}_{32}$-g-APMA - -g-MM $\mathrm{M}_{22}$-g-HM $\mathrm{H}_{43}$ /siRNA polyplexes was five-fold higher compared to the control (free siRNA) at N/P ratio of 4 , while at N/P ratio of 8 cell uptake of polyplex was 11-fold greater compared to control. Confocal microscopy was used to confirm cellular uptake (Figure 7-4D). No detectable siRNA fluorescence was observed in control (free siRNA). In contrast, siRNA fluorescence was clearly seen in the cytoplasm when siRNA was formulated in polymer at N/P ratio of 4 and 8 . The amount of fluorescence was slightly greater at $\mathrm{N} / \mathrm{P}=8$ compared to $\mathrm{N} / \mathrm{P}=4$.

Effect of HPMA 32 -g-APMA 3 -g-MM $\mathrm{MM}_{22}-\mathrm{g}-\mathrm{HM}_{43}$ and Lipofectamine 2000 on AR and XIAP gene silencing

We next examined the effect of charge ratio on AR and XIAP gene silencing in C4-2 cells using $\mathrm{HPMA}_{32}-\mathrm{g}-\mathrm{APMA}_{3}-\mathrm{g}-\mathrm{MM}_{22}-\mathrm{g}-\mathrm{HM}_{43}$ copolymer. We found AR gene silencing to be dependent on charge ratio. Compared to control, AR gene expression decreased to $84.6 \pm 6.7$ and $49.7 \pm 1.9$ at $\mathrm{N} / \mathrm{P}=4$ and N/P $=8$, respectively. Contrarily, XIAP expression decreased by more than $95 \%$ compared to the control regardless of N/P ratio (Figure 7-5). We also compared AR and XIAP gene silencing using $\mathrm{HPMA}_{32}$-g- 

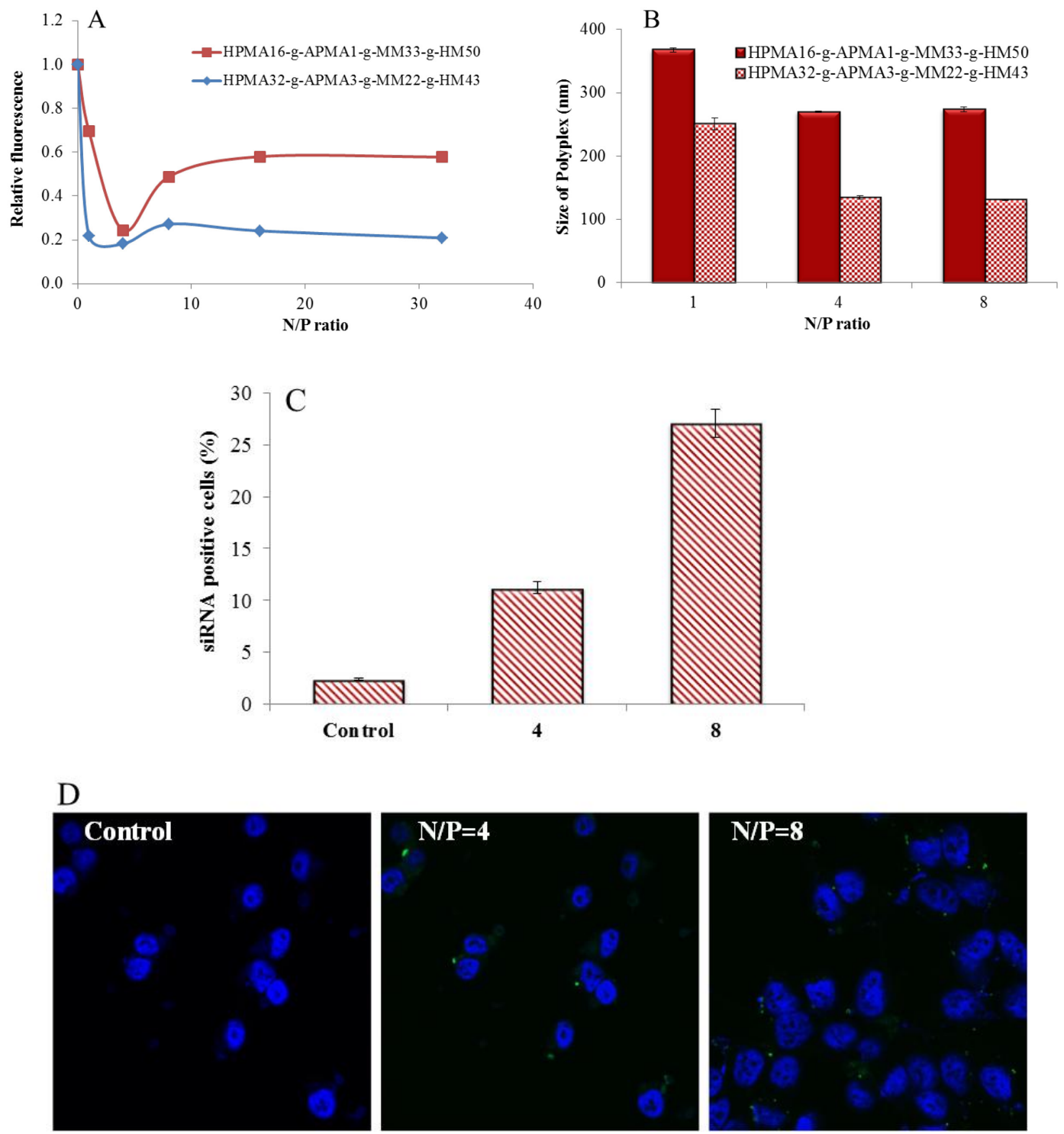

Figure 7-4. Effect of charge (N/P) ratio on siRNA condensation, polyplex size and cellular uptake.

(A) siRNA condensation by HPMA-g-APMA-g-MM-g-HM copolymers in HEPES buffer monitored by ethidium bromide fluorescence quenching assay; (B) size of polyplex and (C and D) cellular uptake of FAM-siRNA HPMA-g-APMA-g-MM-g-HM complexes by $\mathrm{C} 4-2$ cells. siRNA positive cells determined using flow cytometry. Cells treated with naked siRNA served as control. $* \mathrm{P}<0.01$. Confocal microscopy images of cells post treatment with FAM-siRNA formulated with $\mathrm{HPMA}_{32}-\mathrm{g}-\mathrm{APMA}_{3}-\mathrm{g}^{-} \mathrm{MM}_{22}$-g$\mathrm{HM}_{43}$ at N/P ratio of 4 and 8 . Nuclei (blue) are stained with DAPI and siRNA polyplex fluoresce green. Results were represented as the mean $\pm \operatorname{SD}(n=3)$. 


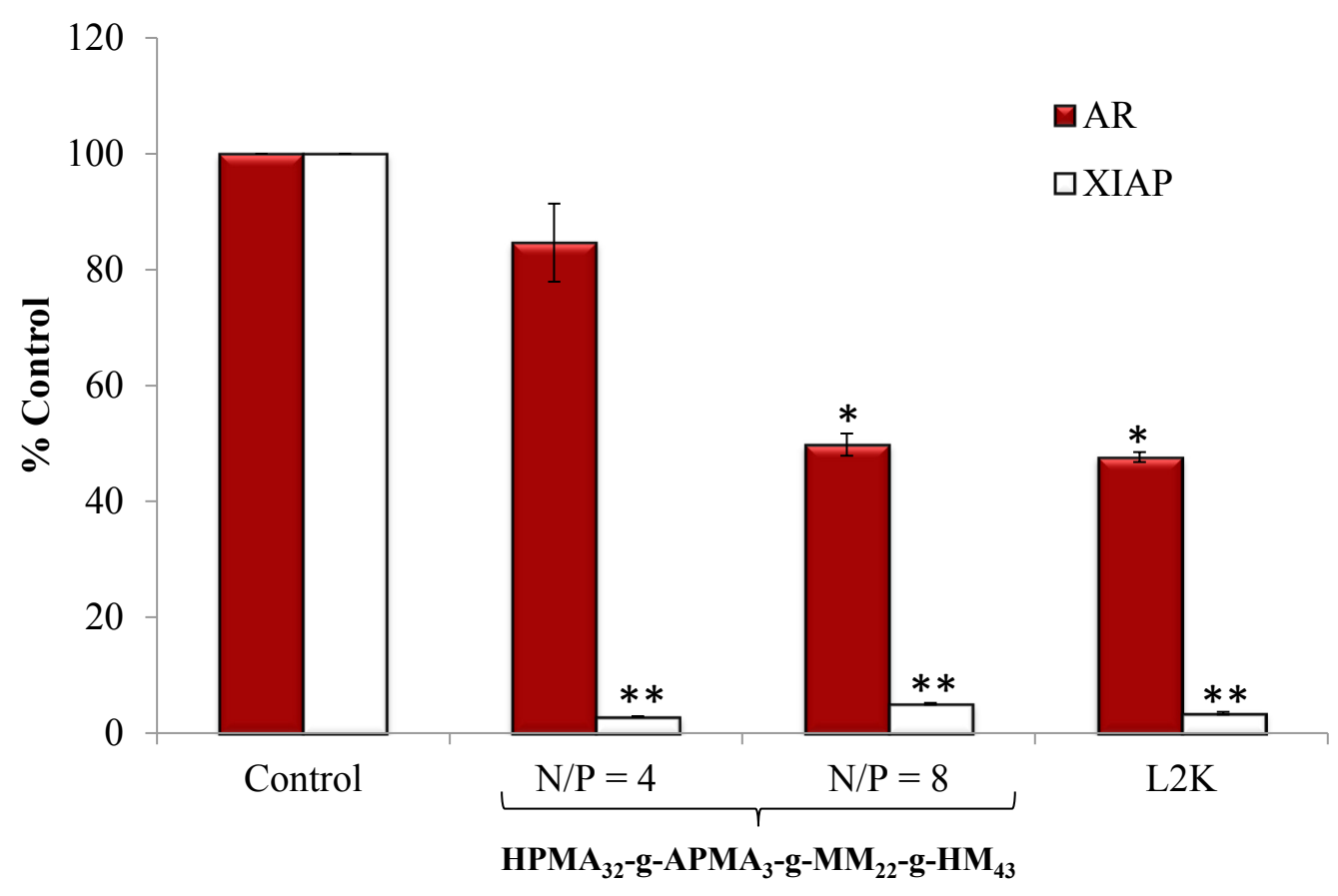

Figure 7-5. Comparison of HPMA $\mathrm{H}_{32}-\mathrm{g}-\mathrm{APMA}_{3}-\mathrm{g}-\mathrm{MM}_{22}-\mathrm{g}-\mathrm{HM} \mathrm{M}_{43}$ and lipofectamine 2000 on AR and XIAP gene silencing.

$\mathrm{HPMA}_{32}$-g-APMA $-\mathrm{g}-\mathrm{MM}_{22}-\mathrm{g}-\mathrm{HM}_{43}(\mathrm{~N} / \mathrm{P}$ ratio $=4$ and 8) and lipofectamine 2000 were used to complex siRNA $(100 \mathrm{nM}) .3 \times 10^{3} \mathrm{C} 4-2$ cells were treated with complexes for 72 $\mathrm{h}$ and extent of gene silencing determined using real-time RT PCR. Results were represented as the mean $\pm \mathrm{SD}(n=3) . * p<0.01, * * p<0.001$. 
observed when siRNA was formulated with $\mathrm{HPMA}_{32}-\mathrm{g}-\mathrm{APMA}_{3}-\mathrm{g}-\mathrm{MM}_{22}-\mathrm{g}-\mathrm{HM}_{43}$ copolymer at $\mathrm{N} / \mathrm{P}=8$ was comparable to that using Lipofectamine 2000.

$\mathrm{AR}$ and XIAP gene silencing induces apoptosis and inhibits $\mathrm{C} 4-2$ cell proliferation

We hypothesized that simultaneous silencing of AR and XIAP should translate into increased apoptosis. Therefore, we examined the effect of AR (1172) and XIAP (264) siRNA, alone or in combination, on apoptotic induction in C4-2 cells after $96 \mathrm{~h}$.

From Figure 7-6A, 1172 was more potent than 264 in inducing apoptosis (43\% vs. 23\%). Also, treatment of C4-2 cells with 1172 and 264 combination resulted in significant increase in percent of cells undergoing apoptosis (85\%) compared to control or monotherapy.

We postulated that the increased apoptosis observed from simultaneously silencing AR and XIAP should result from increase in caspase 3 expression. Therefore, we examined the effect of 1172 and 264, alone or in combination, on caspase 3 mRNA and protein levels. Treatment with 1172 was more potent than 264 in increasing caspase 3 mRNA expression ( 1.3 fold vs. 1.9 fold). However, dual silencing of AR and XIAP led to approximately 7-fold increase in caspase 3 mRNA expression (Figure 7-6C). Western blotting was used to determine effect of 1172 and 264 on caspase 3 protein levels. Consistent with our real time RT-PCR results, 1172 and 264 combination was most effective in inducing apoptosis based on increase in procaspase 3 expression levels compared to control or monotherapy. Treatment with 264 and 1172 silencing resulted in 1.1 and 1.6 fold increase in procaspase 3 levels compared to control. In contrast, simultaneous silencing of AR and XIAP was at least 3.7-fold more potent in increasing caspase 3 protein levels compared to monotherapy (Figure 7-6D).

To examine the effects of AR and XIAP inhibition on cell proliferation, C4-2 cells were transfected with 1172 and 264 alone or in combination and assayed after 96 hours. XIAP and AR down-regulation resulted in approximately $20 \%$ and $50 \%$ growth inhibition in C4-2 cells, respectively, compared to control (Figure 7-6E). However, combined silencing of XIAP and AR genes decreased cell growth to approximately $38 \%$ and was more potent in inhibiting cell proliferation compared to monotherapy.

\section{Construction of shRNA plasmids}

Following the encouraging results obtained using AR and XIAP siRNA, we selected potent siRNA sequences targeting AR and XIAP genes, converted them into shRNA and constructed three shRNA expressing vectors so as to obtain longer silencing and hence better therapeutic effect. pshRNA-297, pshRNA-3008 and pshRNA-3008+297 vectors were obtained by inserting the respective sequences (Table 7-3) into psiRNADUO vector (InvivoGen, San Diego, CA) and plasmids confirmed by DNA sequencing. A schematic representation of the constructed shRNA expression vectors is shown in Figure 7-7. 

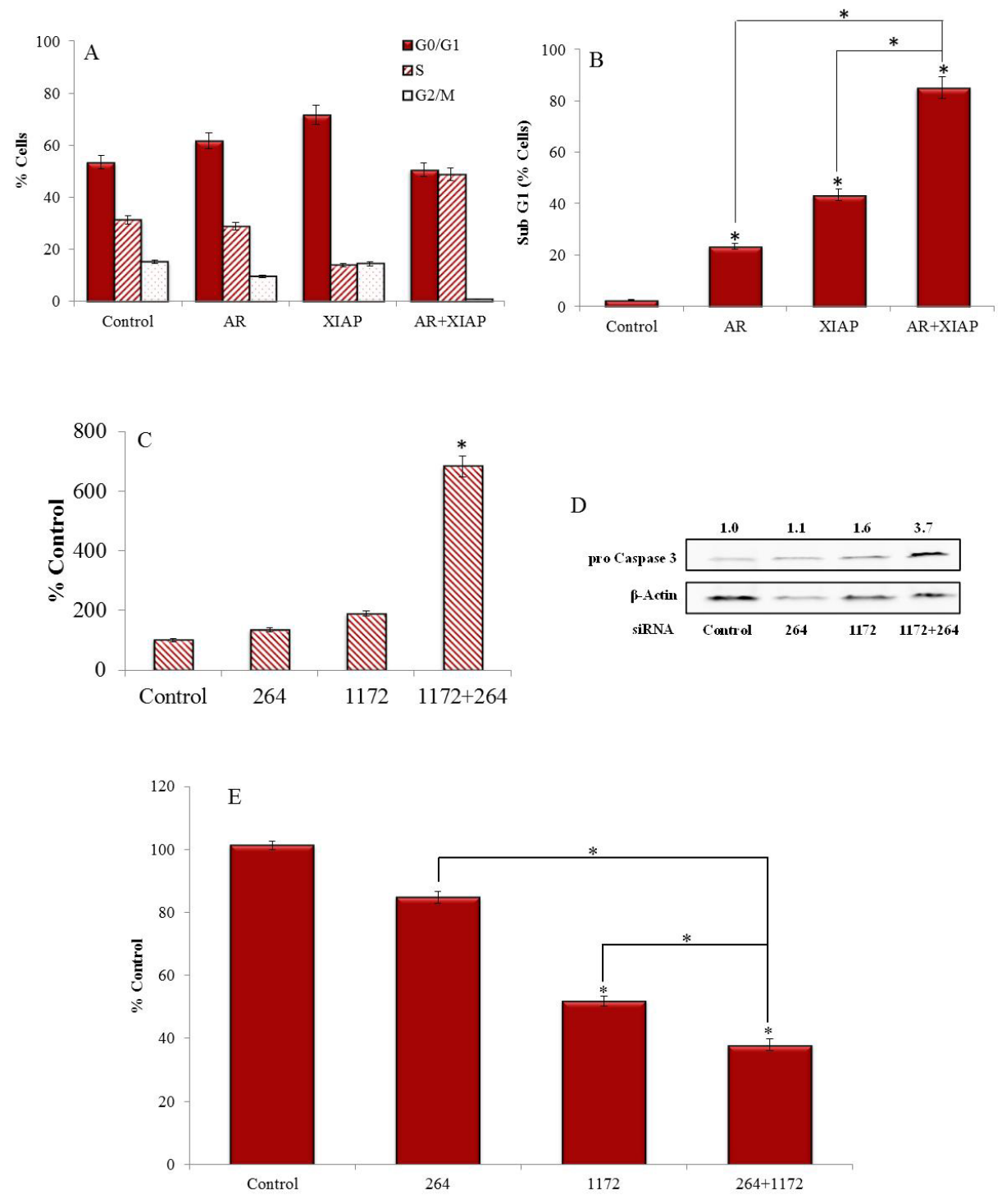

Figure 7-6. Effect of AR and XIAP gene silencing on apoptosis and proliferation in C4-2 cells.

Cells were transfected with siRNA targeting AR(1172) and XIAP (264) alone or in combination for $96 \mathrm{~h}$. Cells were then stained with propidium iodide and analyzed on a flow cytometer. (A) Quantitative analysis of cell cycle distribution. (B) Graphical depiction of apoptotic index. (C) $1 \times 10^{5}$ cells were transfected with siRNA targeting AR(1172) and XIAP (264) alone or in combination for $48 \mathrm{~h}$, total RNA extracted and caspase 3 expression at mRNA level determined using real-time RT-PCR. (D) Western blot analysis of pro-caspase 3 protein expression in C4-2 cells post transfection with siRNA targeting AR(1172) and XIAP (264) alone or in combination for $96 \mathrm{~h}$. (E) Cell viability was determined by MTT assay and expressed as \% of control. Results were represented as the mean $\pm \mathrm{SD}(n=3) .{ }^{*} p<0.01$ compared to control. 

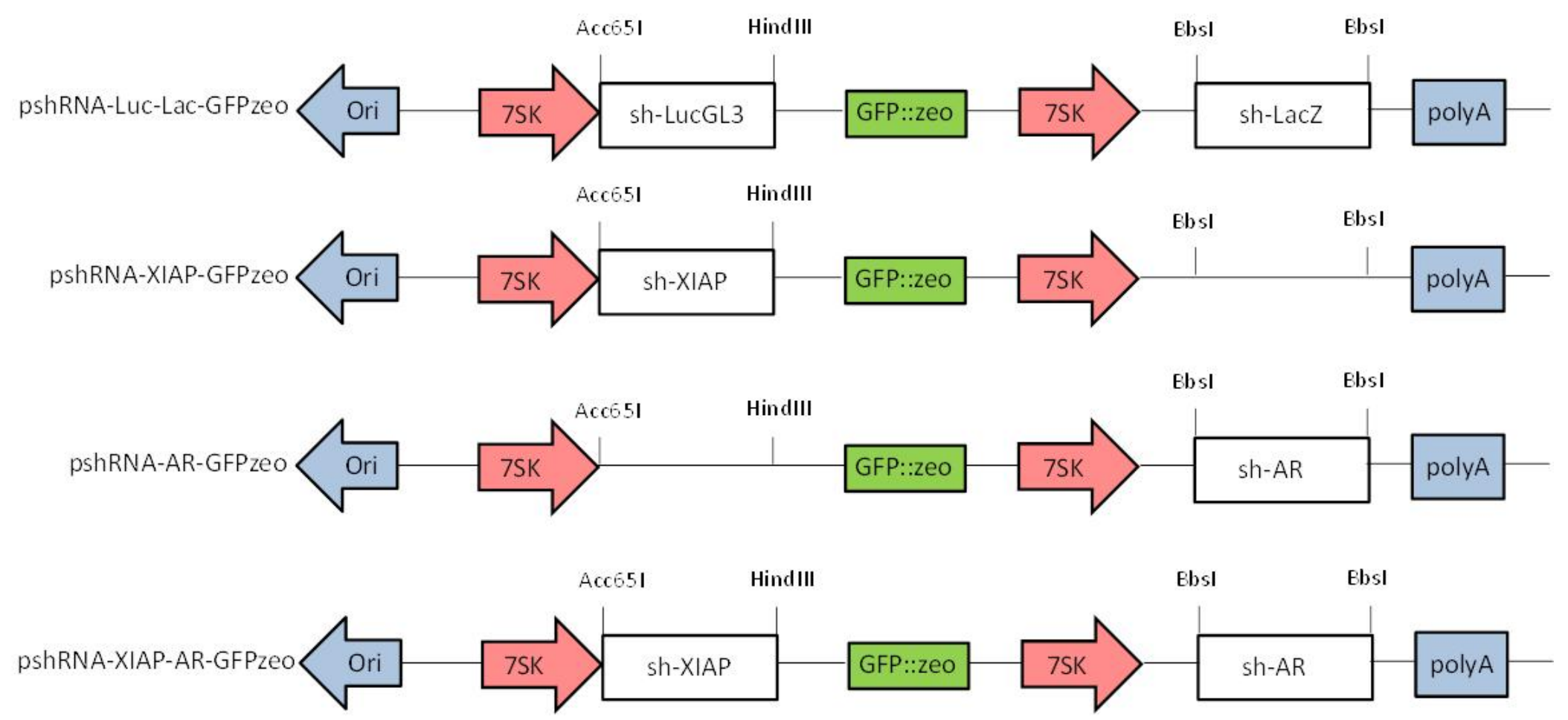

Figure 7-7. Schematic representation of shRNA expression vectors encoding single shRNAs targeting AR or XIAP or bipartite vector encoding shRNA simultaneously targeting AR and XIAP driven by two 7SK promoters. 
Effect of $\mathrm{HPMA}_{32}$-g-APMA $-\mathrm{g}-\mathrm{MM}_{22}$-g-HM $\mathrm{H}_{43}$ copolymer on plasmid transfection efficiency and condensation

Following construction of shRNA plasmids, we determined the ability of $\mathrm{HPMA}_{32}$-g-APMA 3 -g- $\mathrm{MM}_{22}$-g-HM $\mathrm{H}_{43}$ to transfect these vectors into C4-2 cells using fluorescent microscopy. For this study, C4-2 cells were engineered to express red fluorescent protein (RFP) while the shRNA vector express green fluorescent protein (GFP). Using our optimized conditions, we were able to obtain $\sim 70 \%$ transfection efficiency with $\mathrm{HPMA}_{32}$-g-APMA $-\mathrm{g}-\mathrm{MM}_{22}-\mathrm{g}-\mathrm{HM}_{43}(\mathrm{~N} / \mathrm{P}=8)$ copolymer as depicted by the presence of green and orange cells (Figure 7-8A).

We also determined the ability of $\mathrm{HPMA}_{32}-\mathrm{g}-\mathrm{APMA}_{3}-\mathrm{g}-\mathrm{MM}_{22}-\mathrm{g}-\mathrm{HM}_{43}$ copolymer to condense our shRNA vector using the ethidium bromide assay. As shown in Figure 7-8B, $\mathrm{HPMA}_{32}$-g-APMA $-\mathrm{g}-\mathrm{MM}_{22}$-g- $\mathrm{HM}_{43}$ copolymer effectively condensed our shRNA vector as demonstrated by significant ethidium bromide fluorescence quenching even at N/P ratio of 1.

\section{Effect of shRNA sequence on AR gene silencing}

We next examined the effect of shRNA sequence (Table 7-3) on AR gene silencing. In particular, we were interested in the effect of the first nucleotide on gene silencing under the 7SK promoter. From Figure 7-9A, pshRNA-1172 and pshRNA-1648 did not result in any detectable AR silencing in C4-2 cells even at $72 \mathrm{~h}$ post transfection. In contrast, pshRNA-3008 resulted in approximately $60 \%$ decrease in AR mRNA expression. This difference is largely due to fact that both pshRNA-1172 and pshRNA1648 have a pyrimidine (cytosine) as the first nucleotide while pshRNA-3008 has a purine (guanine) as the first nucleotide. We also found AR silencing using pshRNA-3008 to be time-dependent. Compared to control, pshRNA-3008 resulted in approximately $25 \%$ silencing after $24 \mathrm{~h}$ compared to approximately $60 \%$ at $72 \mathrm{~h}$ (Figure 7-9B).

Effect of shRNA expression on AR and XIAP gene silencing

To assess the effectiveness of our bipartite plasmid (pshRNA-3008-297), we employed the psiRNA-DUO vector to direct synthesis of AR and XIAP siRNAs in C4-2 prostate cancer cells. Subsequent to pshRNA-3008-297 cloning, amplification and purification, $\mathrm{HPMA}_{32}-\mathrm{g}-\mathrm{APMA}_{3}-\mathrm{g}-\mathrm{MM}_{22}-\mathrm{g}-\mathrm{HM}_{43} /$ plasmid polyplexes were formulated and particle size and zeta potential determined using dynamic light scattering. Polyplexes had a mean particle size of $111 \pm 2.43 \mathrm{~nm}$ and zeta potential of $-2.94 \pm 0.03 \mathrm{mV}$. Compared to the control, XIAP gene silencing in C4-2 cells was $~ 60 \%$ post transfection of pshRNA-3008-297. This level of silencing was similar to that of pshRNA-297 which silences XIAP alone, while pshRNA-3008 has no effect on XIAP expression (Figure 7-10A). Also, both pshRNA-3008 and pshRNA-3008-297 resulted in $\sim 60 \%$ AR silencing whereas pshRNA-297 had no effect (Figure 7-10B). 


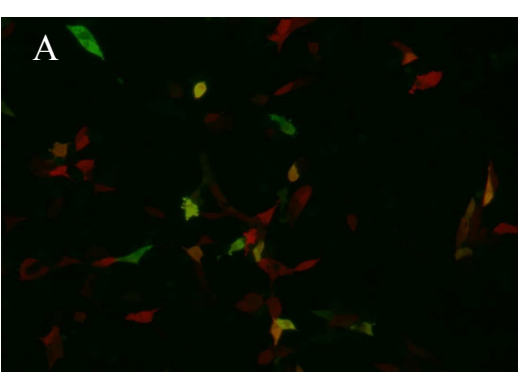

$\mathrm{HPMA}_{32}$-g-APMA $-\mathrm{g}-\mathrm{MM}_{22}-\mathrm{g}-\mathrm{HM}_{43}$ $(\mathrm{N} / \mathrm{P}$ ratio $=8)$

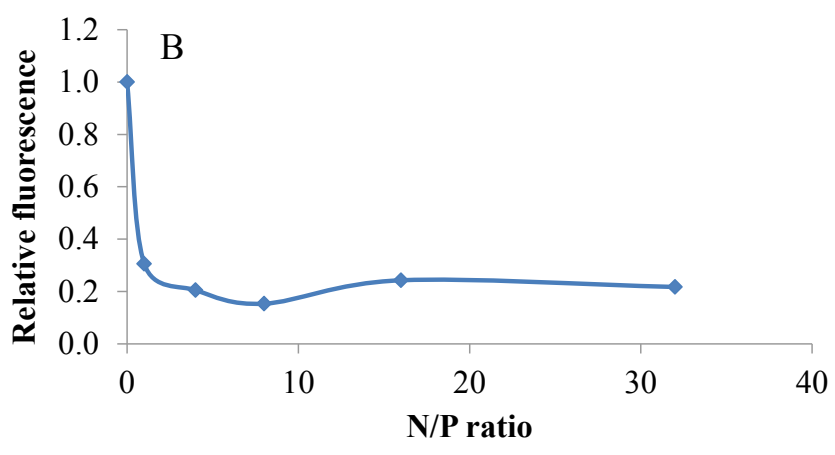

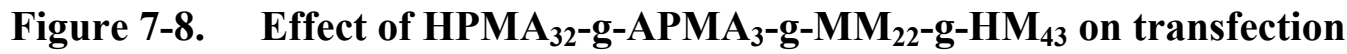
efficiency and condensation.

(A) $\mathrm{HPMA}_{32}-\mathrm{g}-\mathrm{APMA}_{3}-\mathrm{g}-\mathrm{MM}_{22}-\mathrm{g}-\mathrm{HM}_{43}(\mathrm{~N} / \mathrm{P}$ ratio $=8)$ was used to complex pshRNAGFP $(1 \mu \mathrm{g})$. C4-2 cells were treated with complexes for $72 \mathrm{~h}$ and extent of extent of transfection determined fluorescent microscopy. (B) Effect of charge (N/P) ratio on plasmid condensation by HPMA-APMA-lipid copolymers in HEPES buffer monitored by ethidium bromide fluorescence quenching assay. 

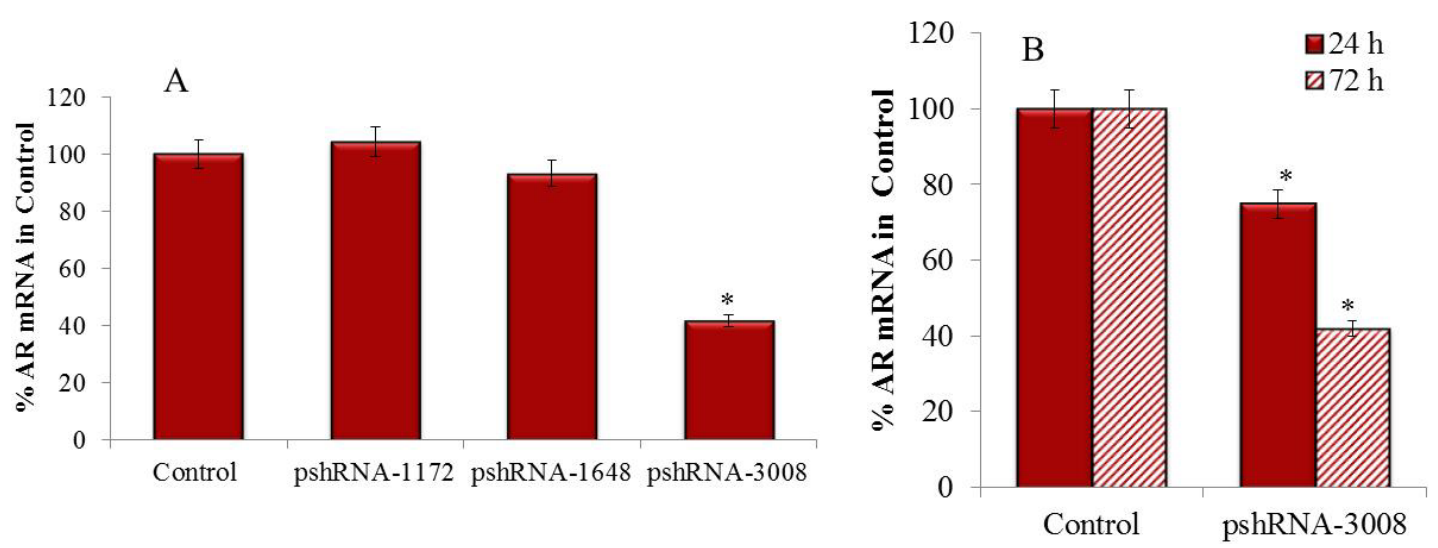

Figure 7-9. Effect of shRNA sequence and time on androgen receptor (AR) gene silencing.

(A) Effect of shRNA sequence on androgen receptor (AR) gene silencing. Three different siRNA sequences targeting specified regions of the AR were converted into shRNA expression vectors and their ability to silence AR gene was examined in C4-2 prostate cancer $72 \mathrm{~h}$ post transfection. (B) Time dependent effect of pshRNA-3008 on AR gene silencing in C4-2 cells. Results were represented as the mean $\pm \mathrm{SD}(n=3)$. $* \mathrm{p}<0.01$ using student's unpaired t- test. 

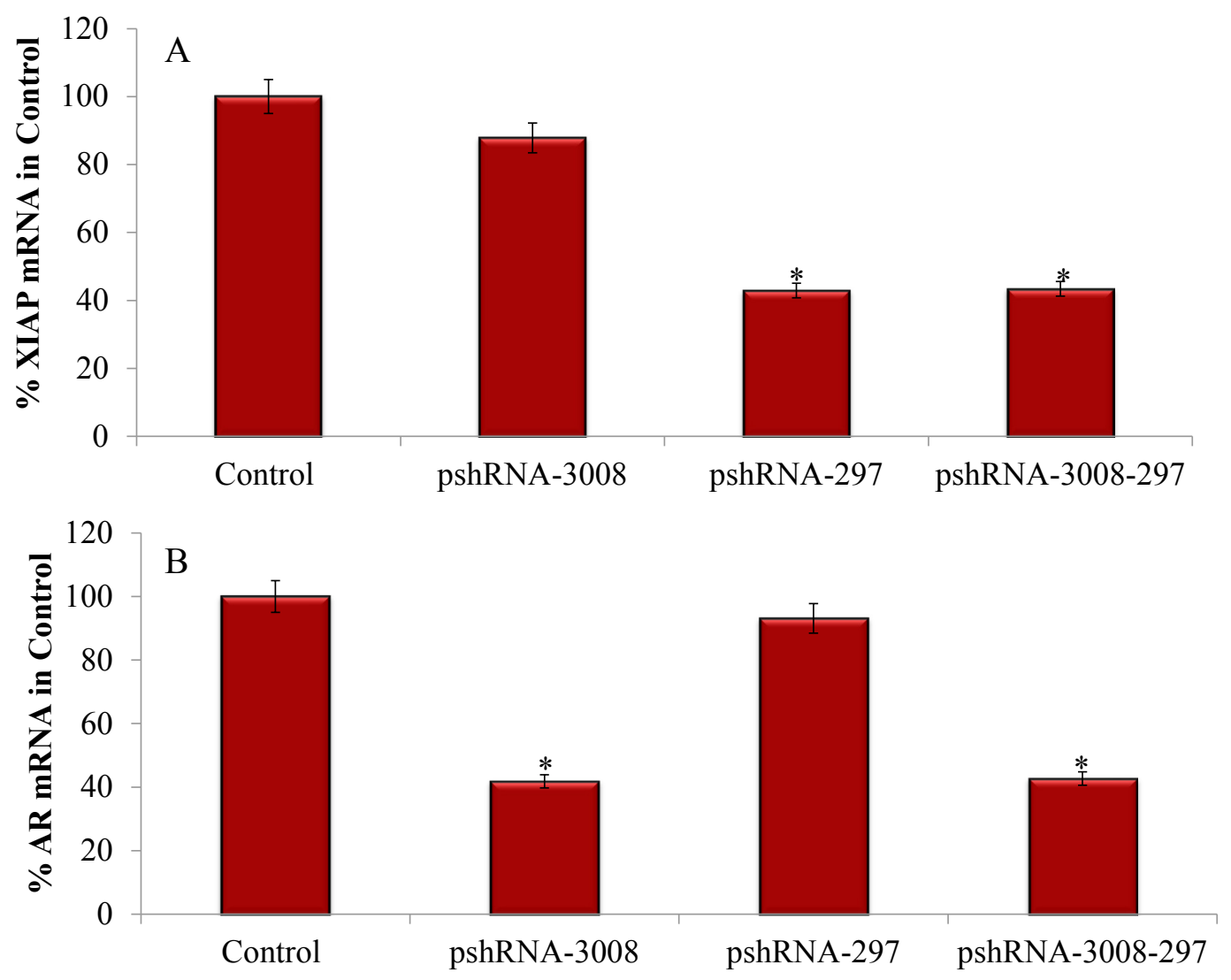

Figure 7-10. Effect of shRNA expression on AR and XIAP gene silencing.

(A) Effect of control plasmid, pshRNA-3008, pshRNA-297 and pshRNA-3008-297 vectors on XIAP gene expression. (B) Effect of control plasmid, pshRNA-3008, pshRNA-297 and pshRNA-3008-297 vectors on AR gene expression. C4-2 cells were treated with complexes for $72 \mathrm{~h}$ and extent of gene silencing determined using real-time RT PCR. Results were represented as the mean \pm SD $(n=3)$. ${ }^{*} \mathrm{p}<0.01$ using student's unpaired t- test. 


\section{Discussion}

Prostate cancer is a highly prevalent and lethal disease affecting men in the United States, with one in six men likely to be diagnosed with the disease in their life time. The predominant nature of prostate cancer requires the development of new therapeutic paradigms due to the limitations of existing treatments. Current anticancer drugs are limited to certain groups of receptors, enzymes and ion channels and are typically susceptible to chemoresistance. However, the genetic disposition of prostate cancer makes it amenable to RNAi-mediated therapy. RNAi molecules (e.g., siRNA, shRNA) interfere with translation, do not interact with chromosomal DNA and are not subject to chemoresistance. Among all genes, AR and XIAP are pivotal mediators of prostate cancer and targeting these genes has attracted much attention. Therefore, the goal of this study was to determine whether simultaneously targeting AR and XIAP using chemical synthesized siRNAs and vector based shRNAs encapsulated within polymeric nano vehicles can be effective in treating prostate cancer.

Silencing potency of siRNA sequences heavily influences their therapeutic efficacy. Unlike antisense technology, functionality of siRNAs strongly depends on siRNA-specific features rather than local mRNA target characteristics; therefore, we designed siRNA sequences using BLOCK-iT RNAi Designer from Life Technologies and selected three sequences targeting the mRNA of AR and XIAP genes (Table 7-1). Our findings suggest siRNA targeting 1172 coding site for AR exhibited the best silencing effect regardless of cell type. Also, all siRNAs targeting XIAP mRNA (264, 297 and 458 coding sites) resulted in strong gene silencing in C4-2 cells. The excellent gene silencing observed suggest these siRNAs have good assembly with the RNA induces silencing complex (RISC), adequately activate RISC and exhibit strong target recognition and cleavage [307].

Although we used Lipofectamine 2000 for screening siRNA sequences, one major goal of this work was to develop a comparatively less toxic delivery system for RNAi molecules. Therefore, we synthesized and evaluated a biodegradable non-toxic HPMA based multi component copolymer for nucleic acid delivery capable of overcoming the barriers (e.g., poor cellular uptake) limiting siRNA delivery to mRNA target sites. To achieve this, three distinct moieties: polycationic (APMA), lipid (HM) and pka modulator (MM) were grafted onto the HPMA backbone. The presence of primary amines in the APMA group allows electrostatic interaction with siRNA and vector based shRNAs thereby neutralizing the negative charge and facilitating condensation. Since cell membranes are composed of lipids, it is hypothesized that inclusion of lipid chains such as HM can facilitate transfection efficiency and cellular uptake. Furthermore, inclusion of the pka modulator is expected to aid siRNA release in the endosome. This is important since siRNA therapy can only be effective if it is released in a timely fashion when delivered.

Our findings reveal this polymeric carrier effectively condensed siRNA and vector based shRNA into stable complexes. Also, polyplexes resulted in enhanced cellular uptake, transfection efficiency and gene silencing at optimum charge (N/P) 
ratios. Biophysical characterization indicated formation of $\mathrm{HPMA}_{32}-\mathrm{g}-\mathrm{APMA}_{3}-\mathrm{g}-\mathrm{MM}_{22}-$ $\mathrm{g}-\mathrm{HM}_{43}$ polyplexes was influenced by N/P ratio. Specifically, polyplex size decreased with increasing N/P ratio. This is likely due to stronger electrostatic interaction between copolymer and siRNA as N/P increases [308]. Furthermore, there was a positive correlation between N/P ratio and gene silencing. This may result from the formation of more stable complexes and the improved cellular uptake of polyplexes.

Our previous studies suggest combination therapy targeting AR and XIAP to be a promising approach for treating prostate cancer. Therefore, we were interested in determining whether AR and XIAP combination therapy using RNA interference would result in a similar phenomenon. To test our combination therapy we selected siRNAs targeting 1172 and 297 coding sites. Indeed, we found simultaneous silencing of AR and XIAP genes translated into supra-additive induction of apoptosis and increased inhibition of cell proliferation compared to monotherapy or control. Interestingly, silencing of XIAP alone resulted in comparatively lower apoptosis compared to AR silencing. Hence, it is possible that $\mathrm{AR}$ gene silencing serves as an apoptotic trigger whereas XIAP gene silencing relieves an apoptotic molecular brake. This result is therefore consistent with previously reported work where we observed similar supra additive effects using small molecule anticancer agents $[11,279]$.

Vector-based shRNAs are a promising means of achieving gene silencing since they are comparatively cheaper than siRNAs and more stable in vivo. Therefore, to demonstrate the utility of our delivery system and to confirm our combination therapy hypothesis, we successfully constructed a bipartite plasmid simultaneously targeting AR and XIAP genes using the most potent sequences identified in the siRNA screening process. Interestingly, we observed shRNA targeting AR mRNA had poor silencing effect which is likely due to the first nucleotide being a pyrimidine (cytosine). Subsequent change to a sequence targeting the 3008 coding site possessing a purine (guanine) resulted in approximately $60 \%$ silencing of AR after $72 \mathrm{~h}$. Furthermore, gene silencing using our bipartite system was specific and comparable to that obtained with the single insert (Figure 7-9). Our $\mathrm{HPMA}_{32}$-g-APMA ${ }_{3}$-g- $\mathrm{MM}_{22}$-g-HM $\mathrm{H}_{43}$ carrier was also observed to effectively condense vector based shRNAs.

In summary, we have described the synthesis, characterization and in vitro assessment of biodegradable HPMA based copolymer consisting of polycationic, lipid and pka modulator moieties for efficient siRNA and vector based shRNA delivery. We showed that this copolymer efficiently condensed siRNA and plasmid, enhanced cellular uptake of siRNA and AR and XIAP gene silencing. Additionally, we have demonstrated that simultaneous targeting of AR and XIAP using RNA interference is potent in inhibiting cell proliferation and inducing apoptosis in hormone refractory prostate cancer compared to monotherapy or control. Our findings suggest $\mathrm{HPMA}_{32}$-g-APMA $-\mathrm{g}-\mathrm{MM}_{22}-$ $\mathrm{g}-\mathrm{HM}_{43}$ polyplexes are a promising approach for delivering siRNA or vector based shRNA for treating prostate cancer. However, further in vivo studies are needed to fully demonstrate proof-of-concept and ultimate clinical translation. 


\section{CHAPTER 8. CONCLUSIONS AND FUTURE RESEARCH}

Since the seminal work of Huggins and Hodges [309, 310] regarding the key role of androgen in prostate cancer, therapeutic approaches revolving around decreasing circulating androgen (e.g., use of antiandrogens) have been adopted as the primary mode of treatment. However, it is clear that this treatment strategy is at best palliative. Even recent application of chemotherapy appears to offer little hope to prostate cancer patients. Therefore, there is an urgent need for a paradigm shift concerning efficient and effective treatment modalities for prostate cancer. This dissertation focused on developing and investigating an AR and XIAP pathway-based combination therapy approach for treating prostate cancer and the engineering of polymeric nano vehicles to facilitate delivery of drug and nucleic acid utilized in the therapeutic strategy. The specific conclusions of this study are presented below.

\section{Cancer Therapeutics}

The potential of combination therapy simultaneously targeting the androgen receptor and XIAP pathways has been investigated through a series of in vitro and in vivo studies. Combination therapy using bicalutamide (antiandrogen) and embelin (XIAP inhibitor) was more potent in treating prostate cancer compared to monotherapy or control. Effectiveness of bicalutamide and embelin combination therapy was found to be cell line and schedule dependent. Combination was synergistic in C4-2 cells and for simultaneous and sequential treatment (bicalutamide followed by embelin). Second generation antiandrogen ((S)-N-(4-cyano-3-(trifluoromethyl) phenyl)-3-((4cyanophenyl)(methyl)amino)-2-hydroxy-2-methylpropanamide) (CBDIV17)) was more potent than bicalutamide in inhibiting proliferation of C4-2 and LNCaP cells and in inducing apoptosis. Combination of CBDIV17 and embelin led to supra-additive antiproliferative and apoptotic effects. Embelin was observed to downregulate AR expression and decrease androgen-mediated AR phosphorylation at Ser ${ }^{81}$. Simultaneously targeting AR and XIAP using siRNAs was also more effective in treating prostate cancer compared to monotherapy or control.

\section{Biomaterials and NanoBio Technology}

A series of biocompatible materials with varying chemical and structural properties have been designed and synthesized to improve micelle drug loading, stability and controlled drug release. Specifically, it was established that chemical tailoring of the PEG- $b$-PLLA micelle core through the introduction of carbonate moieties enhanced the solubilization of the highly lipophilic drug bicalutamide. This result was consistent with predictions made based on the Flory-Huggins interaction parameters computed for polymer/bicalutamide pairs using the group contribution method. Bicalutamide loading in PEG- $b$-P(CB-co-LA) micelles was four times better than in PEG- $b$-PLLA. PEG- $b$-P(CB$c o$-LA) copolymers were also shown to significantly solubilize bicalutamide as aqueous 
micellar solutions had drug concentrations approximately 800 -fold higher than the saturated solubility of bicalutamide in water. In water, PEG- $b$-P(CB-co-LA) copolymers formed spherical micelles with CMC values up to 10 times lower than PEG- $b$-PLLA copolymers (i.e., $0.31 \mu \mathrm{M}$ for $20 \mathrm{~mol} \%$ carbonate and $3.3 \mu \mathrm{M}$ for $0 \mathrm{~mol} \%$ carbonate), indicating that introduction of carbonate monomer results in copolymers which are more thermodynamically favored for self-assembly and exhibit better thermodynamic stability. Finally, PEG- $b$-P(CB-co-LA) copolymers was found to result in slower bicalutamide release compared to PEG- $b$-PLLA copolymers. Even though the inhibitory effect of bicalutamide-loaded PEG- $b$-P(CB-co-LA) micelles on LNCaP cell proliferation was similar to free drug these results are quite significant since drug dissolved in methanol would not be suitable for clinical application. In conclusion, these studies highlight the effect of chemically modifying the PEG- $b$-PLLA micelle core with a carbonate monomer on drug loading, release and stability and demonstrate their potential use as drug delivery vehicles.

Further modifications were made to the existing PEG- $b-\mathrm{P}(\mathrm{CB}-c o-\mathrm{LA})$ copolymer by strategically introducing crosslinkable groups in the core and core-corona interface to further enhance micelle stability [311]. A series of complimentary spectroscopic techniques was used to confirm successful synthesis by ring opening polymerization, elucidate polymer microstructure and confirm crosslinking. $\mathrm{mPEG}_{114}-b-\mathrm{PMAC}_{2.5}-b-$ $\mathrm{P}\left(\mathrm{CB}_{9}-c o-\mathrm{LA}_{39}\right)$ and $\mathrm{mPEG}_{114}-b-\mathrm{P}\left(\mathrm{CB}_{8}-c o-\mathrm{LA}_{35}-c o-\mathrm{MAC}_{2.5}\right)$ copolymers for interface and core crosslinking, respectively, displayed low polydispersity (1.08) and CMC values $(0.8-1 \mathrm{mg} / \mathrm{L})$. Inclusion of MAC monomer did not significantly alter micelle size or morphology irrespective of polymer composition or MAC location. Importantly, $\mathrm{mPEG}_{114}-b-\mathrm{P}\left(\mathrm{CB}_{8}-c o-\mathrm{LA}_{35}-c o-\mathrm{MAC}_{2.5}\right)$ had a higher drug encapsulation efficiency $(78.72 \% \pm 0.15)$ compared to $\mathrm{mPEG}_{114}-b-\mathrm{PMAC}_{2.5}-b-\mathrm{P}\left(\mathrm{CB}_{9}-c o-\mathrm{LA}_{39}\right)(20.29 \% \pm 0.11)$. Subsequent micelle crosslinking did not dramatically change drug loading or micelle morphology. However, there was strong link between micelle crosslinking and improved stability when incubated in aqueous media under extensive dilution and physiological simulating serum (BSA $\sim 45 \mathrm{mg} / \mathrm{mL}$ ). Crosslinked micelle size remained unchanged while non-crosslinked micelles disintegrated at a thousand fold dilution. Besides, crosslinked micelle size remained unchanged in BSA whereas there was a timedependent increase in the size of non-crosslinked micelles. These results showed crosslinked micelles to be more stable compared to their non-crosslinked counterparts. Additionally, bicalutamide-loaded crosslinked micelles were found to be more potent in inhibiting proliferation of LNCaP prostate cancer cells compared to non-crosslinked micelles regardless of polymer type. In all, we have demonstrated that these new biodegradable copolymer systems are potentially useful for cancer therapy.

\section{Future Research}

The findings presented in the study demonstrate proof of principle that combination therapy targeting the androgen receptor and XIAP pathways is capable of regressing prostate cancer tumors. It is also evident that rationally designed polymers chemically tailored to solubilize hydrophobic drugs not only enhance drug loading but 
improve overall cancer therapy. Nonetheless, this work is but a modest step towards future polymeric nanomedicine based prostate cancer therapy. Some natural extensions of this work worth consideration are as follows. Future in depth studies elucidating the molecular mechanism governing combination therapy targeting AR and XIAP at the genomic and proteome levels is required. Additionally, investigating the mode of action by which embelin down-regulates AR gene expression is necessary. Such mechanistic insight will not only enhance our understanding but also lead to the design of highly potent molecules.

Micellar delivery platforms envisaged for antiandrogen and XIAP inhibitor combinations should have high drug loading, be crosslinked to ensure excellent in vivo stability and made site-specific to enhance delivery after systemic administration. Regarding polymers for crosslinked micelles, future studies on influence of MAC block length, extent of crosslinking efficiency and ratio of MAC to CB and LA on key micelle properties are required to generate material design rules which can be utilized for customized fabrication of improved micelle delivery platforms. Also, conjugating targeting ligands (e.g., Leutenizing hormone-releasing hormone (LHRH)) to the polymeric micelles to enhance site-specificity is key to obtaining the desired multicomponent micelles for antiandrogen and XIAP inhibitor based combination therapy. Additionally, creating and testing polymers for nucleic acids delivery is required. Possible synthetic approaches include using the lactic acid and carbonate-based chemistries established in this work and systematically grafting polycationic, lipid and pka modulator moieties to facilitate siRNA condensation, transfection efficiency and release, respectively. Finally, futher in vivo testing is necessary to authenticate these findings and facilitate clinical translation. 


\section{LIST OF REFERENCES}

[1] Siegel R, Naishadham D, Jemal A. Cancer statistics, 2012. CA Cancer J Clin. 2012;62:10-29.

[2] Jain RK. Delivery of molecular and cellular medicine to solid tumors. Adv Drug Deliv Rev. 2001;46:149-68.

[3] Allen TM, Cullis PR. Drug delivery systems: entering the mainstream. Science. 2004;303:1818-22.

[4] Liggins RT, Burt HM. Polyether-polyester diblock copolymers for the preparation of paclitaxel loaded polymeric micelle formulations. Adv Drug Deliv Rev. 2002;54:191-202.

[5] Du W, Hong L, Yao T, Yang X, He Q, Yang B, et al. Synthesis and evaluation of water-soluble docetaxel prodrugs-docetaxel esters of malic acid. Bioorg Med Chem. 2007; 15:6323-30.

[6] Luke DR, Kasiske BL, Matzke GR, Awni WM, Keane WF. Effects of cyclosporine on the isolated perfused rat kidney. Transplantation. 1987;43:795-9.

[7] Onetto N, Canetta R, Winograd B, Catane R, Dougan M, Grechko J, et al. Overview of Taxol safety. J Natl Cancer Inst Monogr. 1993:131-9.

[8] Torchilin VP. Recent advances with liposomes as pharmaceutical carriers. Nat Rev Drug Discov. 2005;4:145-60.

[9] Kataoka K, Harada A, Nagasaki Y. Block copolymer micelles for drug delivery: design, characterization and biological significance. Adv Drug Deliv Rev. 2001;47:113-31.

[10] Matsumura Y. Poly (amino acid) micelle nanocarriers in preclinical and clinical studies. Adv Drug Deliv Rev. 2008;60:899-914.

[11] Danquah M, Li F, Duke CB, 3rd, Miller DD, Mahato RI. Micellar delivery of bicalutamide and embelin for treating prostate cancer. Pharm Res. 2009;26:208192.

[12] Greco F, Vicent MJ. Combination therapy: opportunities and challenges for polymer-drug conjugates as anticancer nanomedicines. Adv Drug Deliv Rev. 2009;61:1203-13.

[13] Matsumura Y, Maeda H. A new concept for macromolecular therapeutics in cancer chemotherapy: mechanism of tumoritropic accumulation of proteins and the antitumor agent smancs. Cancer Res. 1986;46:6387-92. 
[14] Maeda H, Matsumura Y. Tumoritropic and lymphotropic principles of macromolecular drugs. Crit Rev Ther Drug Carrier Syst. 1989;6:193-210.

[15] Maeda H, Seymour LW, Miyamoto Y. Conjugates of anticancer agents and polymers: advantages of macromolecular therapeutics in vivo. Bioconjug Chem. 1992;3:351-62.

[16] Jain RK. The next frontier of molecular medicine: delivery of therapeutics. Nat Med. 1998;4:655-7.

[17] Jain RK. Barriers to drug delivery in solid tumors. Sci Am. 1994;271:58-65.

[18] Jain RK. 1995 Whitaker Lecture: delivery of molecules, particles, and cells to solid tumors. Ann Biomed Eng. 1996;24:457-73.

[19] Reinhold HS. Improved microcirculation in irradiated tumours. Eur J Cancer. 1971;7:273-80.

[20] Jain RK. Transport of molecules in the tumor interstitium: a review. Cancer Res. 1987;47:3039-51.

[21] Heldin CH, Rubin K, Pietras K, Ostman A. High interstitial fluid pressure - an obstacle in cancer therapy. Nat Rev Cancer. 2004;4:806-13.

[22] Aukland K, Reed RK. Interstitial-lymphatic mechanisms in the control of extracellular fluid volume. Physiol Rev. 1993;73:1-78.

[23] Gutmann R, Leunig M, Feyh J, Goetz AE, Messmer K, Kastenbauer E, et al. Interstitial hypertension in head and neck tumors in patients: correlation with tumor size. Cancer Res. 1992;52:1993-5.

[24] Milosevic M, Fyles A, Hedley D, Pintilie M, Levin W, Manchul L, et al. Interstitial fluid pressure predicts survival in patients with cervix cancer independent of clinical prognostic factors and tumor oxygen measurements. Cancer Res. 2001;61:6400-5.

[25] Less JR, Posner MC, Boucher Y, Borochovitz D, Wolmark N, Jain RK. Interstitial hypertension in human breast and colorectal tumors. Cancer Res. 1992;52:6371-4.

[26] Milosevic M, Fyles A, Hedley D, Hill R. The human tumor microenvironment: invasive (needle) measurement of oxygen and interstitial fluid pressure. Semin Radiat Oncol. 2004;14:249-58.

[27] Jain RK. Normalization of tumor vasculature: an emerging concept in antiangiogenic therapy. Science. 2005;307:58-62. 
[28] Gullino PM, Grantham FH, Smith SH, Haggerty AC. Modifications of the acidbase status of the internal milieu of tumors. J Natl Cancer Inst. 1965;34:857-69.

[29] Thistlethwaite AJ, Leeper DB, Moylan DJ, 3rd, Nerlinger RE. pH distribution in human tumors. Int J Radiat Oncol Biol Phys. 1985;11:1647-52.

[30] Vaupel P, Kallinowski F, Okunieff P. Blood flow, oxygen and nutrient supply, and metabolic microenvironment of human tumors: a review. Cancer Res. 1989;49:6449-65.

[31] Helmlinger G, Yuan F, Dellian M, Jain RK. Interstitial $\mathrm{pH}$ and $\mathrm{pO} 2$ gradients in solid tumors in vivo: high-resolution measurements reveal a lack of correlation. Nat Med. 1997;3:177-82.

[32] Vaupel P. Physiological properties of malignant tumours. NMR Biomed. 1992;5:220-5.

[33] Vaupel P, Schaefer C, Okunieff P. Intracellular acidosis in murine fibrosarcomas coincides with ATP depletion, hypoxia, and high levels of lactate and total Pi. NMR Biomed. 1994;7:128-36.

[34] Vaupel P, Kelleher DK, Engel T. Stable bioenergetic status despite substantial changes in blood flow and tissue oxygenation in a rat tumour. Br J Cancer. 1994;69:46-9.

[35] Izumi $\mathrm{H}$, Torigoe $\mathrm{T}$, Ishiguchi $\mathrm{H}$, Uramoto $\mathrm{H}$, Yoshida $\mathrm{Y}$, Tanabe $\mathrm{M}$, et al. Cellular $\mathrm{pH}$ regulators: potentially promising molecular targets for cancer chemotherapy. Cancer Treat Rev. 2003;29:541-9.

[36] Vaupel P. Pathophysiology of Solid Tumors. In: Molls M, Vaupel P, Nieder C, Anscher MS, editors. The Impact of Tumor Biology on Cancer Treatment and Multidisciplinary Strategies. Berlin Heidelberg: Springer 2009. p. 52-83.

[37] Yamagata M, Hasuda K, Stamato T, Tannock IF. The contribution of lactic acid to acidification of tumours: studies of variant cells lacking lactate dehydrogenase. Br J Cancer. 1998;77:1726-31.

[38] Helmlinger G, Sckell A, Dellian M, Forbes NS, Jain RK. Acid production in glycolysis-impaired tumors provides new insights into tumor metabolism. Clin Cancer Res. 2002;8:1284-91.

[39] Stubbs M, McSheehy PM, Griffiths JR, Bashford CL. Causes and consequences of tumour acidity and implications for treatment. Mol Med Today. 2000;6:15-9.

[40] Gerweck LE. Tumor $\mathrm{pH}$ : implications for treatment and novel drug design. Semin Radiat Oncol. 1998;8:176-82. 
[41] Bae Y, Jang WD, Nishiyama N, Fukushima S, Kataoka K. Multifunctional polymeric micelles with folate-mediated cancer cell targeting and $\mathrm{pH}$-triggered drug releasing properties for active intracellular drug delivery. Mol Biosyst. 2005;1:242-50.

[42] Bae Y, Nishiyama N, Fukushima S, Koyama H, Yasuhiro M, Kataoka K. Preparation and biological characterization of polymeric micelle drug carriers with intracellular $\mathrm{pH}$-triggered drug release property: tumor permeability, controlled subcellular drug distribution, and enhanced in vivo antitumor efficacy. Bioconjug Chem. 2005;16:122-30.

[43] Lee ES, Na K, Bae YH. Doxorubicin loaded $\mathrm{pH}$-sensitive polymeric micelles for reversal of resistant MCF-7 tumor. J Control Release. 2005;103:405-18.

[44] Mohajer G, Lee ES, Bae YH. Enhanced intercellular retention activity of novel $\mathrm{pH}$-sensitive polymeric micelles in wild and multidrug resistant MCF-7 cells. Pharm Res. 2007;24:1618-27.

[45] Lee ES, Kim D, Youn YS, Oh KT, Bae YH. A virus-mimetic nanogel vehicle. Angew Chem Int Ed Engl. 2008;47:2418-21.

[46] Iyer AK, Khaled G, Fang J, Maeda H. Exploiting the enhanced permeability and retention effect for tumor targeting. Drug Discov Today. 2006;11:812-8.

[47] Hockel M, Vaupel P. Tumor hypoxia: definitions and current clinical, biologic, and molecular aspects. J Natl Cancer Inst. 2001;93:266-76.

[48] Denny WA. Prodrug strategies in cancer therapy. Eur J Med Chem. 2001;36:57795.

[49] Jain RK, Duda DG, Clark JW, Loeffler JS. Lessons from phase III clinical trials on anti-VEGF therapy for cancer. Nat Clin Pract Oncol. 2006;3:24-40.

[50] Greenberg JI, Shields DJ, Barillas SG, Acevedo LM, Murphy E, Huang J, et al. A role for VEGF as a negative regulator of pericyte function and vessel maturation. Nature. 2008;456:809-13.

[51] Carmeliet P, Jain RK. Angiogenesis in cancer and other diseases. Nature. 2000;407:249-57.

[52] Dean M, Fojo T, Bates S. Tumour stem cells and drug resistance. Nat Rev Cancer. 2005;5:275-84.

[53] Zhou S, Schuetz JD, Bunting KD, Colapietro AM, Sampath J, Morris JJ, et al. The ABC transporter Bcrp1/ABCG2 is expressed in a wide variety of stem cells and is a molecular determinant of the side-population phenotype. Nat Med. 2001;7:1028-34. 
[54] Kim M, Turnquist H, Jackson J, Sgagias M, Yan Y, Gong M, et al. The multidrug resistance transporter ABCG2 (breast cancer resistance protein 1) effluxes Hoechst 33342 and is overexpressed in hematopoietic stem cells. Clin Cancer Res. 2002;8:22-8.

[55] Scharenberg CW, Harkey MA, Torok-Storb B. The ABCG2 transporter is an efficient Hoechst 33342 efflux pump and is preferentially expressed by immature human hematopoietic progenitors. Blood. 2002;99:507-12.

[56] Hu L, McArthur C, Jaffe RB. Ovarian cancer stem-like side-population cells are tumourigenic and chemoresistant. Br J Cancer. 2010;102:1276-83.

[57] Steinbach D, Legrand O. ABC transporters and drug resistance in leukemia: was P-gp nothing but the first head of the Hydra? Leukemia. 2007;21:1172-6.

[58] Costello RT, Mallet F, Gaugler B, Sainty D, Arnoulet C, Gastaut JA, et al. Human acute myeloid leukemia CD34+/CD38- progenitor cells have decreased sensitivity to chemotherapy and Fas-induced apoptosis, reduced immunogenicity, and impaired dendritic cell transformation capacities. Cancer Res. 2000;60:4403-11.

[59] Padera TP, Stoll BR, Tooredman JB, Capen D, di Tomaso E, Jain RK. Pathology: cancer cells compress intratumour vessels. Nature. 2004;427:695.

[60] Padera TP, Kadambi A, di Tomaso E, Carreira CM, Brown EB, Boucher Y, et al. Lymphatic metastasis in the absence of functional intratumor lymphatics. Science. 2002;296:1883-6.

[61] Leu AJ, Berk DA, Lymboussaki A, Alitalo K, Jain RK. Absence of functional lymphatics within a murine sarcoma: a molecular and functional evaluation. Cancer Res. 2000;60:4324-7.

[62] Skobe M, Hawighorst T, Jackson DG, Prevo R, Janes L, Velasco P, et al. Induction of tumor lymphangiogenesis by VEGF-C promotes breast cancer metastasis. Nat Med. 2001;7:192-8.

[63] Stacker SA, Caesar C, Baldwin ME, Thornton GE, Williams RA, Prevo R, et al. VEGF-D promotes the metastatic spread of tumor cells via the lymphatics. Nat Med. 2001;7:186-91.

[64] Fukumura D, Jain RK. Tumor microenvironment abnormalities: causes, consequences, and strategies to normalize. J Cell Biochem. 2007;101:937-49.

[65] Isaka N, Padera TP, Hagendoorn J, Fukumura D, Jain RK. Peritumor lymphatics induced by vascular endothelial growth factor-C exhibit abnormal function. Cancer Res. 2004;64:4400-4.

[66] Simionescu N. Cellular aspects of transcapillary exchange. Physiol Rev. 1983;63:1536-79. 
[67] Renkin EM. Cellular and intercellular transport pathways in exchange vessels. Am Rev Respir Dis. 1992;146:S28-31.

[68] Roberts WG, Palade GE. Neovasculature induced by vascular endothelial growth factor is fenestrated. Cancer Res. 1997;57:765-72.

[69] Neal CR, Michel CC. Transcellular gaps in microvascular walls of frog and rat when permeability is increased by perfusion with the ionophore A23187. J Physiol. 1995;488 ( Pt 2):427-37.

[70] Hashizume H, Baluk P, Morikawa S, McLean JW, Thurston G, Roberge S, et al. Openings between defective endothelial cells explain tumor vessel leakiness. Am J Pathol. 2000;156:1363-80.

[71] Rubin P, Casarett G. Microcirculation of tumors. I. Anatomy, function, and necrosis. Clin Radiol. 1966;17:220-9.

[72] Warren BA. The vascular morphology of tumors. In: Peterson HI, editor. Tumor Blood Circulation. Boca Raton: CRC Press; 1979. p. 1-47.

[73] Jain RK. Transport of molecules across tumor vasculature. Cancer Metastasis Rev. 1987;6:559-93.

[74] Takakura Y, Mahato RI, Hashida M. Extravasation of macromolecules. Adv Drug Deliv Rev. 1998;34:93-108.

[75] Groscurth P, Kistler G. [Human renal cell carcinoma in the "nude" mouse: longterm observations (author's transl)]. Beitr Pathol. 1977;160:337-60.

[76] Hirano A, Matsui T. Vascular structures in brain tumors. Hum Pathol. 1975;6:611-21.

[77] Warren BA. The ultrastructure of the microcirculation at the advancing edge of Walker 256 carcinoma. Microvasc Res. 1970;2:443-53.

[78] Majno G, Shea SM, Leventhal M. Endothelial contraction induced by histaminetype mediators: an electron microscopic study. J Cell Biol. 1969;42:647-72.

[79] McDonald DM, Thurston G, Baluk P. Endothelial gaps as sites for plasma leakage in inflammation. Microcirculation. 1999;6:7-22.

[80] Roberts WG, Palade GE. Increased microvascular permeability and endothelial fenestration induced by vascular endothelial growth factor. J Cell Sci. 1995;108 ( Pt 6):2369-79. 
[81] Dvorak HF, Nagy JA, Feng D, Brown LF, Dvorak AM. Vascular permeability factor/vascular endothelial growth factor and the significance of microvascular hyperpermeability in angiogenesis. Curr Top Microbiol Immunol. 1999;237:97132.

[82] Dvorak AM, Kohn S, Morgan ES, Fox P, Nagy JA, Dvorak HF. The vesiculovacuolar organelle (VVO): a distinct endothelial cell structure that provides a transcellular pathway for macromolecular extravasation. J Leukoc Biol. 1996;59:100-15.

[83] Feng D, Nagy JA, Hipp J, Dvorak HF, Dvorak AM. Vesiculo-vacuolar organelles and the regulation of venule permeability to macromolecules by vascular permeability factor, histamine, and serotonin. J Exp Med. 1996;183:1981-6.

[84] Dvorak AM, Feng D. The vesiculo-vacuolar organelle (VVO). A new endothelial cell permeability organelle. J Histochem Cytochem. 2001;49:419-32.

[85] Yuan F. Transvascular drug delivery in solid tumors. Semin Radiat Oncol. 1998;8:164-75.

[86] Rippe B, Haraldsson B. Fluid and protein fluxes across small and large pores in the microvasculature. Application of two-pore equations. Acta Physiol Scand. 1987;131:411-28.

[87] Boucher Y, Baxter LT, Jain RK. Interstitial pressure gradients in tissue-isolated and subcutaneous tumors: implications for therapy. Cancer Res. 1990;50:4478-84.

[88] Jain RK. Vascular and interstitial barriers to delivery of therapeutic agents in tumors. Cancer Metastasis Rev. 1990;9:253-66.

[89] Morikawa S, Baluk P, Kaidoh T, Haskell A, Jain RK, McDonald DM. Abnormalities in pericytes on blood vessels and endothelial sprouts in tumors. Am J Pathol. 2002;160:985-1000.

[90] Tong RT, Boucher Y, Kozin SV, Winkler F, Hicklin DJ, Jain RK. Vascular normalization by vascular endothelial growth factor receptor 2 blockade induces a pressure gradient across the vasculature and improves drug penetration in tumors. Cancer Res. 2004;64:3731-6.

[91] Arap W, Pasqualini R, Ruoslahti E. Cancer treatment by targeted drug delivery to tumor vasculature in a mouse model. Science. 1998;279:377-80.

[92] Hobbs SK, Monsky WL, Yuan F, Roberts WG, Griffith L, Torchilin VP, et al. Regulation of transport pathways in tumor vessels: role of tumor type and microenvironment. Proc Natl Acad Sci U S A. 1998;95:4607-12. 
[93] Yuan F, Dellian M, Fukumura D, Leunig M, Berk DA, Torchilin VP, et al. Vascular permeability in a human tumor xenograft: molecular size dependence and cutoff size. Cancer Res. 1995;55:3752-6.

[94] Provenzale JM, Mukundan S, Dewhirst M. The role of blood-brain barrier permeability in brain tumor imaging and therapeutics. AJR Am J Roentgenol. 2005;185:763-7.

[95] Campbell RB, Fukumura D, Brown EB, Mazzola LM, Izumi Y, Jain RK, et al. Cationic charge determines the distribution of liposomes between the vascular and extravascular compartments of tumors. Cancer Res. 2002;62:6831-6.

[96] Thurston G, McLean JW, Rizen M, Baluk P, Haskell A, Murphy TJ, et al. Cationic liposomes target angiogenic endothelial cells in tumors and chronic inflammation in mice. J Clin Invest. 1998;101:1401-13.

[97] Jain RK. The Eugene M. Landis Award Lecture 1996. Delivery of molecular and cellular medicine to solid tumors. Microcirculation. 1997;4:1-23.

[98] Biedler JL, Riehm H. Cellular resistance to actinomycin D in Chinese hamster cells in vitro: cross-resistance, radioautographic, and cytogenetic studies. Cancer Res. 1970;30:1174-84.

[99] Minko T. HPMA copolymers for modulating cellular signaling and overcoming multidrug resistance. Adv Drug Deliv Rev. 2010;62:192-202.

[100] Yusuf RZ, Duan Z, Lamendola DE, Penson RT, Seiden MV. Paclitaxel resistance: molecular mechanisms and pharmacologic manipulation. Curr Cancer Drug Targets. 2003;3:1-19.

[101] Doyle LA, Ross DD. Multidrug resistance mediated by the breast cancer resistance protein BCRP (ABCG2). Oncogene. 2003;22:7340-58.

[102] van Veen HW, Konings WN. The ABC family of multidrug transporters in microorganisms. Biochim Biophys Acta. 1998;1365:31-6.

[103] Krishna R, Mayer LD. Multidrug resistance (MDR) in cancer. Mechanisms, reversal using modulators of MDR and the role of MDR modulators in influencing the pharmacokinetics of anticancer drugs. Eur J Pharm Sci. 2000;11:265-83.

[104] Maliepaard M, Scheffer GL, Faneyte IF, van Gastelen MA, Pijnenborg AC, Schinkel AH, et al. Subcellular localization and distribution of the breast cancer resistance protein transporter in normal human tissues. Cancer Res. 2001;61:3458-64. 
[105] Doyle LA, Yang W, Abruzzo LV, Krogmann T, Gao Y, Rishi AK, et al. A multidrug resistance transporter from human MCF-7 breast cancer cells. Proc Natl Acad Sci U S A. 1998;95:15665-70.

[106] Minko T, Kopeckova P, Kopecek J. Comparison of the anticancer effect of free and HPMA copolymer-bound adriamycin in human ovarian carcinoma cells. Pharm Res. 1999;16:986-96.

[107] Hu YP, Jarillon S, Dubernet C, Couvreur P, Robert J. On the mechanism of action of doxorubicin encapsulation in nanospheres for the reversal of multidrug resistance. Cancer Chemother Pharmacol. 1996;37:556-60.

[108] Doige CA, Yu X, Sharom FJ. The effects of lipids and detergents on ATPaseactive P-glycoprotein. Biochim Biophys Acta. 1993;1146:65-72.

[109] Kabanov AV, Batrakova EV, Alakhov VY. Pluronic block copolymers for overcoming drug resistance in cancer. Adv Drug Deliv Rev. 2002;54:759-79.

[110] Batrakova EV, Li S, Alakhov VY, Elmquist WF, Miller DW, Kabanov AV. Sensitization of cells overexpressing multidrug-resistant proteins by pluronic P85. Pharm Res. 2003;20:1581-90.

[111] Minko T, Batrakova EV, Li S, Li Y, Pakunlu RI, Alakhov VY, et al. Pluronic block copolymers alter apoptotic signal transduction of doxorubicin in drugresistant cancer cells. J Control Release. 2005;105:269-78.

[112] Batrakova EV, Li S, Brynskikh AM, Sharma AK, Li Y, Boska M, et al. Effects of pluronic and doxorubicin on drug uptake, cellular metabolism, apoptosis and tumor inhibition in animal models of MDR cancers. J Control Release. 2010;143:290-301.

[113] Zhang Y, Tang L, Sun L, Bao J, Song C, Huang L, et al. A novel paclitaxelloaded poly(epsilon-caprolactone)/Poloxamer 188 blend nanoparticle overcoming multidrug resistance for cancer treatment. Acta Biomater. 2010;6:2045-52.

[114] Li PY, Lai PS, Hung WC, Syu WJ. Poly(l-lactide)-Vitamin E TPGS Nanoparticles Enhanced the Cytotoxicity of Doxorubicin in Drug-Resistant MCF7 Breast Cancer Cells. Biomacromolecules. 2010;11:2576-82.

[115] Li F, Lu Y, Li W, Miller DD, Mahato RI. Synthesis, formulation and in vitro evaluation of a novel microtubule destabilizer, SMART-100. J Control Release. 2010;143:151-8.

[116] Lu Y, Li CM, Wang Z, Ross CR, 2nd, Chen J, Dalton JT, et al. Discovery of 4substituted methoxybenzoyl-aryl-thiazole as novel anticancer agents: synthesis, biological evaluation, and structure-activity relationships. J Med Chem. 2009;52:1701-11. 
[117] Shuhendler AJ, O'Brien P, Rauth AM, Wu XY. On the synergistic effects of of doxorubicin and mitomycin $\mathrm{C}$ against breast cancer cells. Drug Metabolism and Drug Interactions. 2008;22:201-33.

[118] Potchen EJ, Kinzie J, Curtis C, Siegel BA, Studer RK. Effect of irradiation on tumor microvascular permeability to macromolecules. Cancer. 1972;30:639-43.

[119] Song CW, Levitt SH. Effect of x irradiation on vascularity of normal tissues and experimental tumor. Radiology. 1970;94:445-7.

[120] Song CW, Sung JH, Clement JJ, Levitt SH. Vascular changes in neuroblastoma of mice following X-irradiation. Cancer Res. 1974;34:2344-50.

[121] Song CW, Levitt SH. Vascular changes in Walker 256 carcinoma of rats following X irradiation. Radiology. 1971;100:397-407.

[122] Gaber MH, Wu NZ, Hong K, Huang SK, Dewhirst MW, Papahadjopoulos D. Thermosensitive liposomes: extravasation and release of contents in tumor microvascular networks. Int J Radiat Oncol Biol Phys. 1996;36:1177-87.

[123] Hauck ML, Dewhirst MW, Bigner DD, Zalutsky MR. Local hyperthermia improves uptake of a chimeric monoclonal antibody in a subcutaneous xenograft model. Clin Cancer Res. 1997;3:63-70.

[124] Engin K. Biological rationale for hyperthermia in cancer treatment (II). Neoplasma. 1994;41:277-83.

[125] Dewhirst MW. Future directions in hyperthermia biology. Int J Hyperthermia. 1994;10:339-45.

[126] Fujiwara K, Watanabe T. Effects of hyperthermia, radiotherapy and thermoradiotherapy on tumor microvascular permeability. Acta Pathol Jpn. 1990;40:79-84.

[127] Lefor AT, Makohon S, Ackerman NB. The effects of hyperthermia on vascular permeability in experimental liver metastasis. J Surg Oncol. 1985;28:297-300.

[128] Hosono MN, Hosono M, Endo K, Ueda R, Onoyama Y. Effect of hyperthermia on tumor uptake of radiolabeled anti-neural cell adhesion molecule antibody in small-cell lung cancer xenografts. J Nucl Med. 1994;35:504-9.

[129] Schuster JM, Zalutsky MR, Noska MA, Dodge R, Friedman HS, Bigner DD, et al. Hyperthermic modulation of radiolabelled antibody uptake in a human glioma xenograft and normal tissues. Int J Hyperthermia. 1995;11:59-72. 
[130] Hauck ML, Coffin DO, Dodge RK, Dewhirst MW, Mitchell JB, Zalutsky MR. A local hyperthermia treatment which enhances antibody uptake in a glioma xenograft model does not affect tumour interstitial fluid pressure. Int J Hyperthermia. 1997;13:307-16.

[131] Kong G, Braun RD, Dewhirst MW. Characterization of the effect of hyperthermia on nanoparticle extravasation from tumor vasculature. Cancer Res. 2001;61:302732 .

[132] Wilhelm DL, Mason B. Vascular permeability changes in inflammation: the role of endogenous permeability factors in mild thermal injury. Br J Exp Pathol. 1960;41:487-506.

[133] Ellerby HM, Arap W, Ellerby LM, Kain R, Andrusiak R, Rio GD, et al. Anticancer activity of targeted pro-apoptotic peptides. Nat Med. 1999;5:1032-8.

[134] Chen Y, Xu X, Hong S, Chen J, Liu N, Underhill CB, et al. RGD-Tachyplesin inhibits tumor growth. Cancer Res. 2001;61:2434-8.

[135] Curnis F, Sacchi A, Borgna L, Magni F, Gasparri A, Corti A. Enhancement of tumor necrosis factor alpha antitumor immunotherapeutic properties by targeted delivery to aminopeptidase N (CD13). Nat Biotechnol. 2000;18:1185-90.

[136] Netti PA, Hamberg LM, Babich JW, Kierstead D, Graham W, Hunter GJ, et al. Enhancement of fluid filtration across tumor vessels: implication for delivery of macromolecules. Proc Natl Acad Sci U S A. 1999;96:3137-42.

[137] Presta LG, Chen H, O'Connor SJ, Chisholm V, Meng YG, Krummen L, et al. Humanization of an anti-vascular endothelial growth factor monoclonal antibody for the therapy of solid tumors and other disorders. Cancer Res. 1997;57:4593-9.

[138] Senger DR, Galli SJ, Dvorak AM, Perruzzi CA, Harvey VS, Dvorak HF. Tumor cells secrete a vascular permeability factor that promotes accumulation of ascites fluid. Science. 1983;219:983-5.

[139] Lee CG, Heijn M, di Tomaso E, Griffon-Etienne G, Ancukiewicz M, Koike C, et al. Anti-Vascular endothelial growth factor treatment augments tumor radiation response under normoxic or hypoxic conditions. Cancer Res. 2000;60:5565-70.

[140] Willett CG, Boucher Y, di Tomaso E, Duda DG, Munn LL, Tong RT, et al. Direct evidence that the VEGF-specific antibody bevacizumab has antivascular effects in human rectal cancer. Nat Med. 2004;10:145-7.

[141] Hurwitz HI, Fehrenbacher L, Hainsworth JD, Heim W, Berlin J, Holmgren E, et al. Bevacizumab in combination with fluorouracil and leucovorin: an active regimen for first-line metastatic colorectal cancer. J Clin Oncol. 2005;23:3502-8. 
[142] Pietras K. Increasing tumor uptake of anticancer drugs with imatinib. Semin Oncol. 2004;31:18-23.

[143] Pietras K, Ostman A, Sjoquist M, Buchdunger E, Reed RK, Heldin CH, et al. Inhibition of platelet-derived growth factor receptors reduces interstitial hypertension and increases transcapillary transport in tumors. Cancer Res. 2001;61:2929-34.

[144] Pietras K, Stumm M, Hubert M, Buchdunger E, Rubin K, Heldin CH, et al. STI571 enhances the therapeutic index of epothilone B by a tumor-selective increase of drug uptake. Clin Cancer Res. 2003;9:3779-87.

[145] Lammerts E, Roswall P, Sundberg C, Gotwals PJ, Koteliansky VE, Reed RK, et al. Interference with TGF-beta1 and -beta3 in tumor stroma lowers tumor interstitial fluid pressure independently of growth in experimental carcinoma. Int J Cancer. 2002;102:453-62.

[146] Salnikov AV, Roswall P, Sundberg C, Gardner H, Heldin NE, Rubin K. Inhibition of TGF-beta modulates macrophages and vessel maturation in parallel to a lowering of interstitial fluid pressure in experimental carcinoma. Lab Invest. 2005;85:512-21.

[147] Salnikov AV, Iversen VV, Koisti M, Sundberg C, Johansson L, Stuhr LB, et al. Lowering of tumor interstitial fluid pressure specifically augments efficacy of chemotherapy. FASEB J. 2003;17:1756-8.

[148] Griffon-Etienne G, Boucher Y, Brekken C, Suit HD, Jain RK. Taxane-induced apoptosis decompresses blood vessels and lowers interstitial fluid pressure in solid tumors: clinical implications. Cancer Res. 1999;59:3776-82.

[149] Taghian AG, Abi-Raad R, Assaad SI, Casty A, Ancukiewicz M, Yeh E, et al. Paclitaxel decreases the interstitial fluid pressure and improves oxygenation in breast cancers in patients treated with neoadjuvant chemotherapy: clinical implications. J Clin Oncol. 2005;23:1951-61.

[150] Maeda H, Sawa T, Konno T. Mechanism of tumor-targeted delivery of macromolecular drugs, including the EPR effect in solid tumor and clinical overview of the prototype polymeric drug SMANCS. Journal of Controlled Release. 2001;74:47-61.

[151] Vasey PA, Kaye SB, Morrison R, Twelves C, Wilson P, Duncan R, et al. Phase I clinical and pharmacokinetic study of PK1 [N-(2-hydroxypropyl)methacrylamide copolymer doxorubicin]: first member of a new class of chemotherapeutic agentsdrug-polymer conjugates. Cancer Research Campaign Phase I/II Committee. Clin Cancer Res. 1999;5:83-94. 
[152] Minko T, Kopeckova P, Pozharov V, Kopecek J. HPMA copolymer bound adriamycin overcomes MDR1 gene encoded resistance in a human ovarian carcinoma cell line. J Control Release. 1998;54:223-33.

[153] Kopecek J, Kopeckova P, Minko T, Lu Z. HPMA copolymer-anticancer drug conjugates: design, activity, and mechanism of action. Eur J Pharm Biopharm. 2000;50:61-81.

[154] Chipman SD, Oldham FB, Pezzoni G, Singer JW. Biological and clinical characterization of paclitaxel poliglumex (PPX, CT-2103), a macromolecular polymer-drug conjugate. Int J Nanomedicine. 2006;1:375-83.

[155] Singer JW. Paclitaxel poliglumex (XYOTAX, CT-2103): a macromolecular taxane. J Control Release. 2005;109:120-6.

[156] Lavasanifar A, Samuel J, Kwon GS. Poly(ethylene oxide)-block-poly(1-amino acid) micelles for drug delivery. Advanced Drug Delivery Reviews. 2002;54:16990.

[157] Yamamoto Y, Nagasaki Y, Kato Y, Sugiyama Y, Kataoka K. Long-circulating poly(ethylene glycol)-poly(D,L-lactide) block copolymer micelles with modulated surface charge. J Control Release. 2001;77:27-38.

[158] Maeda H, Wu J, Sawa T, Matsumura Y, Hori K. Tumor vasculature permeability and the EPR effect in macromolecular therapeutics: a review. Journal of Controlled Release. 2000;65:271-84.

[159] Gadelle F, Koros W, Schechter R. Solubilization of aromatic solutes in block copolymers. Macromolecules. 1995;28:4883-92.

[160] Nagarajan R, Barry M, Ruckenstein E. Unusual selectivity in solubilization by block copolymer micelles. Langmuir. 1986;2:210-5.

[161] Xing L, Mattice W. Strong solubilization of small molecules by triblockcopolymer micelles in selective solvents. Macromolecules. 1997;30:1711-7.

[162] Tian M, Arca E, Tuzar Z, Webber S, Munk P. Light scattering study of solubilization of organic molecules by block copolymer micelles in aqueous media. J Polym Sci, Part B: Pol Phys. 1995;33:1713-22.

[163] Zhang Y, Taiming L, Liu J. Low temperature and glucose enhanced T7 RNA polymerase-based plasmid stability for increasing expression of glucagon-like peptide-2 in Escherichia coli. Protein Expr Purif. 2003;29:132-9.

[164] Latere Dwan'Isa JP, Rouxhet L, Preat V, Brewster ME, Arien A. Prediction of drug solubility in amphiphilic di-block copolymer micelles: the role of polymerdrug compatibility. Pharmazie. 2007;62:499-504. 
[165] Huynh L, Grant J, Leroux JC, Delmas P, Allen C. Predicting the solubility of the anti-cancer agent docetaxel in small molecule excipients using computational methods. Pharm Res. 2008;25:147-57.

[166] Mahmud A, Patel S, Molavi O, Choi P, Samuel J, Lavasanifar A. Self-Associating Poly(ethylene oxide)-b-poly(alpha-cholesteryl carboxylate-epsilon-caprolactone) Block Copolymer for the Solubilization of STAT-3 Inhibitor Cucurbitacin I. Biomacromolecules. 2009.

[167] Forster A, Hempenstall J, Tucker I, Rades T. Selection of excipients for melt extrusion with two poorly water-soluble drugs by solubility parameter calculation and thermal analysis. Int J Pharm. 2001;226:147-61.

[168] Marsac PJ, Shamblin SL, Taylor LS. Theoretical and practical approaches for prediction of drug-polymer miscibility and solubility. Pharm Res. 2006;23:241726.

[169] Liu J, Xiao Y, Allen C. Polymer-Drug Compatibility: A Guide to the Development of Delivery Systems for the Anticancer Agent, Ellipticine. Journal of Pharmaceutical Sciences. 2003;93:132-43.

[170] Danquah M, Fujiwara T, Mahato RI. Self-assembling methoxypoly(ethylene glycol)-b-poly(carbonate-co-L-lactide) block copolymers for drug delivery. Biomaterials. 2010;31:2358-70.

[171] Li F, Danquah M, Mahato RI. Synthesis and Characterization of Amphiphilic Lipopolymers for Micellar Drug Delivery. Biomacromolecules. 2010.

[172] Shuai X, Ai H, Nasongkla N, Kim S, Gao J. Micellar carriers based on block copolymers of poly(-caprolactone) and poly(ethylene glycol) for doxorubicin delivery. J Control Release. 2004;98:415-26.

[173] Shuai X, Merdan T, Schaper A, Xi F, Kissel T. Core-cross-linked polymeric micelles as paclitaxel carriers. Bioconjugate Chem. 2004;15:441-8.

[174] Jemal A, Siegel R, Ward E, Hao Y, Xu J, T.Murray, et al. Cancer statistics, 2008. A Cancer Journal for Clinicians. 2008;58:71-96.

[175] Huggins C, Hodges CV. Studies on prostatic cancer. I. The Effect of Castration, of Estrogen and of Androgen Injection on Serum Phosphatases in Metastatic Carcinoma of the Prostate. Cancer Research. 1941;1:293-7.

[176] Cunha GR, Donjacour AA, Cooke PS, Mee S, Bigsby RM, Higgind SJ, et al. The endocrinology and developmental biology of the prostate. Endocrine Reviews. 1987;8:338-62.

[177] Isaacs JT. Role of androgens in prostate cancer. Vitamins and Hormones. 1994;49:433-502. 
[178] Arnold JT, Isaacs JT. Mechanisms involved in the progression of androgenindependent prostate cnacers: it is not only the cancer cell's fault. EndocrineRelated Cancer. 2002;9:61-73.

[179] Miquel M, Soler A, Vaque A, Ojanguren I, Costa J, Planas R. Suspected crosshepatotoxicity of flutamide and cyproterone acetate. Liver Int. 2007;27:1144-7.

[180] de Voogt HJ, Smith PH, Pavone-Macaluso M, de Pauw M, Suciu S. Cardiovascular side effects of diethylstilbestrol, cyproterone acetate, medroxyprogesterone acetate and estramustine phosphate used for the treatment of advanced prostatic cancer: results from European Organization for Research on Treatment of Cancer trials 30761 and 30762. J Urol. 1986;135:303-7.

[181] Blackledge G. Casodex-mechanism of action and opportunity for usage. Cancer. 1993;72:3830-3.

[182] Deveraux QL, Reed JC. IAP family proteins--suppressors of apoptosis. Genes Dev. 1999;13:239-52.

[183] Kasof GM, Gomes BC. Livin, a novel inhibitor of apoptosis protein family member. J Biol Chem. 2001;276:3238-46.

[184] Salvesen GS, Duckett CS. IAP proteins: blocking the road to death's door. Nat Rev Mol Cell Biol. 2002;3:401-10.

[185] Takahashi R, Deveraux Q, Tamm I, Welsh K, Assa-Munt N, Salvesen GS, et al. A single BIR domain of XIAP sufficient for inhibiting caspases. J Biol Chem. 1998;273:7787-90.

[186] Fesik SW. Insights into programmed cell death through structural biology. Cell. 2000; 103:273-82.

[187] Riedl SJ, Renatus M, Schwarzenbacher R, Zhou Q, Sun C, Fesik SW, et al. Structural basis for the inhibition of caspase-3 by XIAP. Cell. 2001;104:791-800.

[188] Chai J, Shiozaki E, Srinivasula SM, Wu Q, Datta P, Alnemri ES, et al. Structural basis of caspase-7 inhibition by XIAP. Cell. 2001;104:769-80.

[189] McEleny KR, Watson RW, Coffey RN, O'Neill AJ, Fitzpatrick JM. Inhibitors of apoptosis proteins in prostate cancer cell lines. Prostate. 2002;51:133-40.

[190] Krajewska M, Krajewski S, Banares S, Huang X, Turner B, Bubendorf L, et al. Elevated expression of inhibitor of apoptosis proteins in prostate cancer. Clin Cancer Res. 2003;9:4914-25. 
[191] Nikolovska-Coleska Z, Xu L, Hu Z, Tomita Y, Li P, Roller PP, et al. Discovery of embelin as a cell-permeable, small-molecular weight inhibitor of XIAP through structure-based computational screening of a traditional herbal medicine threedimensional structure database. J Med Chem. 2004;47:2430-40.

[192] Chitra M, Sukumar E, Suja V, Devi CS. Antitumor, anti-inflammatory and analgesic property of embelin, a plant product. Chemotherapy. 1994;40:109.

[193] Githui EK, Makawiti DW, Midiwo JO. Changes in the concentrations of testosterone, luteinising hormone and progesterone associated with administration of embelin. Contraception. 1991;44:311-7.

[194] Ahn KS, Sethi G, Aggarwal BB. Embelin, an inhibitor of X chromosome-linked inhibitor-of-apoptosis protein, blocks nuclear factor-kappaB (NF-kappaB) signaling pathway leading to suppression of NF-kappaB-regulated antiapoptotic and metastatic gene products. Mol Pharmacol. 2007;71:209-19.

[195] Maeda H. The enhanced permeability and retention (EPR) effect in tumor vasculature: the key role of tumor-selective macromolecular drug targeting. Adv Enzyme Regul. 2001;41:189-207.

[196] Mukherjee A, Kirkovsky L, Yao XT, Yates RC, Miller DD, Dalton JT. Enantioselective binding of Casodex to the androgen receptor. Xenobiotica. 1996;26:117-22.

[197] Steel GG, Peckham MJ. Exploitable mechanisms in combined radiotherapychemotherapy: the concept of additivity. Int J Radiat Oncol Biol Phys. 1979;5:8591.

[198] Ananthapadmanabhan KP, Goddard ED, Turro NJ, KuoS PL. Fluorescence Probes for Critical Micelle Concentration. Langmuir. 1985;1:352 - 5.

[199] Ham JS. A New Electronic State in Benzene. The Journal of Chemical Physics. $1953 ; 21: 756-8$.

[200] Wilihelm M, Zhao CL, Wang Y, Xu R, Winnik MA. Poly(styrene-ethylene oxide) block copolymer micelle formation in water: A fluorescence probe study. Macromolecules. 1991;24:1033-40.

[201] Blanco E, Bey EA, Dong Y, Weinberg BD, Sutton DM, Boothman DA, et al. bLapachone-containing PEG-PLA polymer micelles as novel nanotherapeutics against NQO1-overexpressing tumor cells. Journal of Controlled Release. 2007;122:365-74.

[202] Grossmann ME, Huang H, Tindall DJ. Androgen receptor signaling in androgenrefractory prostate cancer. J Natl Cancer Inst. 2001;93:1687-97. 
[203] Kish JA, Bukkapatnam R, Palazzo F. The treatment challenge of hormonerefractory prostate cancer. Cancer Control. 2001;8:487-95.

[204] Knox JJ, Moore MJ. Treatment of hormone refractory prostate cancer. Semin Urol Oncol. 2001;19:202-11.

[205] Rubben H, Bex A, Otto T. Systemic treatment of hormone refractory prostate cancer. World J Urol. 2001;19:99-110.

[206] Emamaullee JA, Shapiro AM. Interventional strategies to prevent beta-cell apoptosis in islet transplantation. Diabetes. 2006;55:1907-14.

[207] Lee EC, Zhan P, Schallhom R, Packman K, Tenniswood M. Antiandrogeninduced cell death in LNCaP human prostate cancer cells. Cell Death Differ. 2003;10:761-71.

[208] Liao X, Tang S, Thrasher JB, Griebling TL, Li B. Small-interfering RNA-induced androgen receptor silencing leads to apoptotic cell death in prostate cancer. Mol Cancer Ther. 2005;4:505-15.

[209] Yoshida T, Kinoshita H, Segawa T, Nakamura E, Inoue T, Shimizu Y, et al. Antiandrogen bicalutamide promotes tumor growth in a novel androgendependent prostate cancer xenograft model derived from a bicalutamide-treated patient. Cancer Res. 2005;65:9611-6.

[210] Fakler M, Loder S, Vogler M, Schneider K, Jeremias I, Debatin KM, et al. Small molecule XIAP inhibitors cooperate with TRAIL to induce apoptosis in childhood acute leukemia cells and overcome Bcl-2-mediated resistance. Blood. 2008.

[211] Akutsu M, Kano Y, Tsunoda S, Suzuki K, Yazawa Y, Miura Y. Scheduledependent interaction between paclitaxel and doxorubicin in human cancer cell lines in vitro. Eur J Cancer. 1995;31A:2341-6.

[212] Yang X, Zhu B, Dong T, Pan P, Shuai X, Inoue Y. Interactions between an anticancer drug and polymeric micelles based on biodegradable polyesters. Macromol Biosci. 2008;8:1116-25.

[213] Sirotnak FM, She Y, Lee F, Chen J, Scher HI. Studies with CWR22 xenografts in nude mice suggest that ZD1839 may have a role in the treatment of both androgen-dependent and androgen-independent human prostate cancer. Clin Cancer Res. 2002;8:3870-6.

[214] Burich RA, Holland WS, Vinall RL, Tepper C, White RW, Mack PC. Genistein combined polysaccharide enhances activity of docetaxel, bicalutamide and Src kinase inhibition in androgen-dependent and independent prostate cancer cell lines. BJU Int. 2008;102:1458-66. 
[215] Zhang S, Hsieh ML, Zhu W, Klee GG, Tindall DJ, Young CY. Interactive effects of triiodothyronine and androgens on prostate cell growth and gene expression. Endocrinology. 1999; 140:1665-71.

[216] Blackledge G. Casodex-mechanisms of action and opportunities for usage. Cancer. 1993;72:3830-3.

[217] Hennenfent KL, Govindan R. Novel formulations of taxanes: a review. Old wine in a new bottle? Ann Oncol. 2006;17:735-49.

[218] Otsuka H, Nagasaki Y, Kataoka K. PEGylated nanoparticles for biological and pharmaceutical applications. Adv Drug Deliv Rev. 2003;55:403-19.

[219] Yokoyama M, Miyauchi M, Yamada N, Okano T, Sakurai Y, Kataoka K, et al. Characterization and anticancer activity of the micelle-forming polymeric anticancer drug adriamycin-conjugated poly(ethylene glycol)-poly(aspartic acid) block copolymer. Cancer Res. 1990;50:1693-700.

[220] Yoo HS, Park TG. Biodegradable polymeric micelles composed of doxorubicin conjugated PLGA-PEG block copolymer. J Control Release. 2001;70:63-70.

[221] Liu J, Zeng F, Allen C. In vivo fate of unimers and micelles of a poly(ethylene glycol)-block-poly(caprolactone) copolymer in mice following intravenous administration. Eur J Pharm Biopharm. 2007;65:309-19.

[222] Ramaswamy M, Zhang X, Burt HM, Wasan KM. Human plasma distribution of free paclitaxel and paclitaxel associated with diblock copolymers. J Pharm Sci. 1997;86:460-4.

[223] Frazza EJ, Schmitt EE. A new absorbable suture. J Biomed Mater Res. 1971;5:4358.

[224] Hench LL, Polak JM. Third-generation biomedical materials. Science. 2002;295:1014-7.

[225] Uhrich KE, Cannizzaro SM, Langer RS, Shakesheff KM. Polymeric systems for controlled drug release. Chem Rev. 1999;99:3181-98.

[226] Schaffer JH. Journal of European Patent. 1988;82:107247.

[227] Wang XL, Zhuo RX, Liu LJ, He F, Liu G. Synthesis and Characterization of Novel Aliphatic Polycarbonates. Journal of Polymer Science Part A: Polymer Chemistry. 2002;40:70-5.

[228] Zhu KJ, Hendren RW, Jensen KJ, Pitt CG. Synthesis, Properties and Biodegradation of Poly(1,3-trimethylene Carbonate). Macromolecules. 1991;24:1736-40. 
[229] Watanabe J, Kotera H, Akashi M. Reflective Interfaces of Poly(trimethylene Carbonate)-Based Polymers: Enzymatic Degradation and Selective Adsorption. Macromolecules. 2007;40:8731-6.

[230] Danquah M, Li F, Duke CB, 3rd, Miller DD, Mahato RI. Micellar Delivery of Bicalutamide and Embelin for Treating Prostate Cancer. Pharmaceutical Research. 2009;26:2081-92.

[231] Pratt RC, Nederberg F, Waymouth RM, Hedrick JL. Tagging alcohols with cyclic carbonate: a versatile equivalent of (meth)acrylate for ring-opening polymerization. Chem Commun (Camb). 2008:114-6.

[232] Morton M, Kaizerman S, Altier MW. Swelling of latex particles. Journal of Colloidal Science. 1954;9:300.

[233] Guan H, Xie Z, Tang Z, Xu X, Chen X, Jing X. Preparation of Block Copolymer of $\varepsilon$-Caprolactone and 2-methyl-2-carboxyl-propylene carbonate. Polymer. 2004;46:2817.

[234] Xie Z, Hu X, Chen X, Sun J, Shi Q, Jing X. Synthesis and characterization of novel biodegradable poly(carbonate ester)s with photolabile protecting groups. Biomacromolecules. 2008;9:376-80.

[235] Guan H, Xie Z, Zhang P, Wang X, Chen X, Wang X, et al. Synthesis and Characterization of Novel Biodegradable Block Copolymer Poly(ethylene glycol)-block-poly(L-lactide-co-2-methyl-2-carboxyl-propylene carbonate). Journal of Polymer Science Part A: Polymer Chemistry. 2005;43:4771-80.

[236] Al-Azemi TF, Bisht KS. Novel Functional Polycarbonate by Lipase-Catalyzed Ring-Opening Polymeization of 5-Methyl-5-benzyloxcarbonyl-1,3-dioxan-2-one. Macromolecules. 1999;32:6536-40.

[237] Tsuji H, Ikada Y. Stereocomplex formation between enantiomeric poly(lactic acid)s. 6. Binary blends from copolymers. Macromolecules. 1992;25:5719-23.

[238] Ray WC, Grinstaff MW. Polycarbonate and Poly(carbonate-ester)s Synthesized from Biocompatible Building Blocks of Glycerol and Lactic Acid. Macromolecules. 2002;36:3557-62.

[239] Jemal A, Siegel R, Xu J, Ward E. Cancer statistics, 2010. CA Cancer J Clin. 2010;60:277-300.

[240] Gittes RF. Carcinoma of the prostate. N Engl J Med. 1991;324:236-45.

[241] Kozlowski JM, Ellis WJ, Grayhack JT. Advanced prostatic carcinoma. Early versus late endocrine therapy. Urol Clin North Am. 1991;18:15-24. 
[242] Bracarda S, de Cobelli O, Greco C, Prayer-Galetti T, Valdagni R, Gatta G, et al. Cancer of the prostate. Crit Rev Oncol Hematol. 2005;56:379-96.

[243] Goktas S, Crawford ED. Optimal hormonal therapy for advanced prostatic carcinoma. Semin Oncol. 1999;26:162-73.

[244] Scher HI, Sawyers CL. Biology of progressive, castration-resistant prostate cancer: directed therapies targeting the androgen-receptor signaling axis. J Clin Oncol. 2005;23:8253-61.

[245] Taplin ME, Balk SP. Androgen receptor: a key molecule in the progression of prostate cancer to hormone independence. J Cell Biochem. 2004;91:483-90.

[246] Danquah MK, Zhang XA, Mahato RI. Extravasation of polymeric nanomedicines across tumor vasculature. Adv Drug Deliv Rev. 2011;63:623-39.

[247] Li F, Danquah M, Mahato RI. Synthesis and characterization of amphiphilic lipopolymers for micellar drug delivery. Biomacromolecules. 2010;11:2610-20.

[248] Li F, Danquah M, Singh S, Wu H, Mahato RI. Paclitaxel- and lapatinib-loaded lipopolymer micelles overcome multidrug resistance in prostate cancer. Drug Delivery and Translational Research. 2011;1:240-8.

[249] Sun C, Shi Y, Xu LL, Nageswararao C, Davis LD, Segawa T, et al. Androgen receptor mutation (T877A) promotes prostate cancer cell growth and cell survival. Oncogene. 2006;25:3905-13.

[250] Wu Y, Chhipa RR, Zhang H, Ip C. The antiandrogenic effect of finasteride against a mutant androgen receptor. Cancer biology \& therapy. 2011;11:902-9.

[251] Chen J, Nikolovska-Coleska Z, Wang G, Qiu S, Wang S. Design, synthesis, and characterization of new embelin derivatives as potent inhibitors of X-linked inhibitor of apoptosis protein. Bioorg Med Chem Lett. 2006;16:5805-8.

[252] Liu S, Yuan Y, Okumura Y, Shinkai N, Yamauchi H. Camptothecin disrupts androgen receptor signaling and suppresses prostate cancer cell growth. Biochem Biophys Res Commun. 2010;394:297-302.

[253] Ren F, Zhang S, Mitchell SH, Butler R, Young CY. Tea polyphenols downregulate the expression of the androgen receptor in LNCaP prostate cancer cells. Oncogene. 2000;19:1924-32.

[254] Chen L, Meng S, Wang H, Bali P, Bai W, Li B, et al. Chemical ablation of androgen receptor in prostate cancer cells by the histone deacetylase inhibitor LAQ824. Mol Cancer Ther. 2005;4:1311-9. 
[255] Purushottamachar P, Khandelwal A, Chopra P, Maheshwari N, Gediya LK, Vasaitis TS, et al. First pharmacophore-based identification of androgen receptor down-regulating agents: discovery of potent anti-prostate cancer agents. Bioorg Med Chem. 2007;15:3413-21.

[256] Lecis D, Drago C, Manzoni L, Seneci P, Scolastico C, Mastrangelo E, et al. Novel SMAC-mimetics synergistically stimulate melanoma cell death in combination with TRAIL and Bortezomib. Br J Cancer. 2010;102:1707-16.

[257] Huerta S, Gao X, Livingston EH, Kapur P, Sun H, Anthony T. In vitro and in vivo radiosensitization of colorectal cancer HT-29 cells by the smac mimetic JP-1201. Surgery. 2010;148:346-53.

[258] Dai Y, Desano J, Qu Y, Tang W, Meng Y, Lawrence TS, et al. Natural IAP inhibitor Embelin enhances therapeutic efficacy of ionizing radiation in prostate cancer. Am J Cancer Res. 2011;1:128-43.

[259] Kwon GS. Polymeric micelles for delivery of poorly water-soluble compounds. Critical reviews in therapeutic drug carrier systems. 2003;20:357-403.

[260] Soga O, van Nostrum CF, Fens M, Rijcken CJ, Schiffelers RM, Storm G, et al. Thermosensitive and biodegradable polymeric micelles for paclitaxel delivery. J Control Release. 2005;103:341-53.

[261] Torchilin VP. Micellar nanocarriers: pharmaceutical perspectives. Pharm Res. 2007;24:1-16.

[262] Solink S, Illum L, Davus SS. Long circulating microparticulate drug carriers. Adv Drug Deliv Rev. 1995;16:195-214.

[263] Bronich TK, Keifer PA, Shlyakhtenko LS, Kabanov AV. Polymer micelle with cross-linked ionic core. J Am Chem Soc. 2005;127:8236-7.

[264] Lavasanifar A, Samuel J, Kwon GS. Poly(ethylene oxide)-block-poly(L-amino acid) micelles for drug delivery. Advanced drug delivery reviews. 2002;54:16990.

[265] Liu J, Zeng F, Allen C. Influence of serum protein on polycarbonate-based copolymer micelles as a delivery system for a hydrophobic anti-cancer agent. J Control Release. 2005;103:481-97.

[266] Savic R, Azzam T, Eisenberg A, Maysinger D. Assessment of the integrity of poly(caprolactone)-b-poly(ethylene oxide) micelles under biological conditions: a fluorogenic-based approach. Langmuir. 2006;22:3570-8.

[267] Rosler A, Vandermeulen GW, Klok HA. Advanced drug delivery devices via selfassembly of amphiphilic block copolymers. Adv Drug Deliv Rev. 2001;53:95108. 
[268] Joralemon MJ, Murthy KS, Remsen EE, Becker ML, Wooley KL. Synthesis, characterization, and bioavailability of mannosylated shell cross-linked nanoparticles. Biomacromolecules. 2004;5:903-13.

[269] O'Reilly RK, Hawker CJ, Wooley KL. Cross-linked block copolymer micelles: functional nanostructures of great potential and versatility. Chemical Society reviews. 2006;35:1068-83.

[270] Read ES, Armes SP. Recent advances in shell cross-linked micelles. Chem Commun (Camb). 2007:3021-35.

[271] Hawker CJ, Wooley KL. The convergence of synthetic organic and polymer chemistries. Science. 2005;309:1200-5.

[272] Iijima M, Nagasaki Y, Okada T, Kato M, Kataoka K. Core-Polymerized Reactive Micelles from Heterotelechelic Amphiphilic Block Copolymers. Macromolecules. 1999;32:1140-6.

[273] Kim J-H, Emoto K, Iijima M, Nagasaki Y, Aoyagi T, Okano T, et al. Corestabilized Polymeric Micelle as Potential Drug Carrier: Increased Solubilization of Taxol. Polymers for Advanced Technologies. 1999;10:647-54.

[274] Hu X, Chen X, Xie Z, Liu S, Jing X. Synthesis and Characterization of Amphiphilic Block Copolymers with Allyl Side-Groups. Journal of Polymer Science: Part A: Polymer Chemistry. 2007;45:5518-28.

[275] Garg SM, Xiong X-B, Lu C, Lavasanifar A. Application of Click Chemistry in the Preparation of Poly(ethylene oxide)-block-poly( $\varepsilon$-caprolactone) with Hydrolyzable Cross-Links in the Micellar Core. Macromolecules. 2011;44:205866.

[276] Butun V, Wang X-S, de Paz Banez MV, Robinson KL, Billingham NC, Armes SP, et al. Synthesis of Shell Cross-Linked Micelles at High Solids in Aqueous Media. Macromolecules. 1999;33:1-3.

[277] Hu X, Chen X, Wei J, Liu S, Jing X. Core crosslinking of biodegradable block copolymer micelles based on poly(ester carbonate). Macromol Biosci. 2009;9:456-63.

[278] Yang R, Meng F, Ma S, Huang F, Liu H, Zhong Z. Galactose-decorated crosslinked biodegradable poly(ethylene glycol)-b-poly(epsilon-caprolactone) block copolymer micelles for enhanced hepatoma-targeting delivery of paclitaxel. Biomacromolecules. 2011;12:3047-55.

[279] Danquah M, Duke CB, 3rd, Patil R, Miller DD, Mahato RI. Combination therapy of antiandrogen and XIAP inhibitor for treating advanced prostate cancer. Pharm Res. 2012;29:2079-91. 
[280] Li F, Danquah M, Singh S, Wu H, Mahato RI. Paclitaxel- and lapatinib-loaded lipopolymer micelles overcome multidrug resistance in prostate cancer. Drug Delivery and Translational Research. 2011;1:420-8.

[281] Liu J, Xiao Y, Allen C. Polymer-drug compatibility: A guide to the development of delivery systems for the anticancer agent, Ellipticine. Journal of Pharmaceutical Sciences. 2004;93:132-43.

[282] Chen W, Yang H, Wang R, Cheng R, Meng F, Wei W, et al. Versatile Synthesis of Functional Biodegradable Polymers by Combining Ring-Opening Polymerization and Postpolymerization Modification via Michael-Type Addition Reaction. Macromolecules. 2010;43:201-7.

[283] Kaljurand I, Kutt A, Soovali L, Rodima T, Maemets V, Leito I, et al. Extension of the self-consistent spectrophotometric basicity scale in acetonitrile to a full span of $28 \mathrm{pKa}$ units: unification of different basicity scales. The Journal of organic chemistry. 2005;70:1019-28.

[284] Watanabe J, Amemori S, Akashi M. Disparate polymerization facilitates the synthesis of versatile block copolymers from poly(trimethylene carbonate). Polymer. 2008;49:3709-15.

[285] Qian H, Wohl AR, Crow JT, Macosko CW, Hoye TR. A Strategy for Control of "Random" Copolymerization of Lactide and Glycolide: Application to Synthesis of PEG-b-PLGA Block Polymers Having Narrow Dispersity. Macromolecules. 2011;44:7132-40.

[286] Lohmeijer BGG, Pratt RC, Leibfarth F, Logan JW, Long DA, Dove AP, et al. Guanidine and amidine organo-catalysts for ring-opening polymerization of cyclic esters. Macromolecules. 2006;39:8574-83.

[287] Kim S, Shi Y, Kim JY, Park K, Cheng JX. Overcoming the barriers in micellar drug delivery: loading efficiency, in vivo stability, and micelle-cell interaction. Expert Opin Drug Deliv. 2010;7:49-62.

[288] Attwood D, Booth C, Yeates SG, Chaibundit C, Ricardo NM. Block copolymers for drug solubilisation: relative hydrophobicities of polyether and polyester micelle-core-forming blocks. International journal of pharmaceutics. 2007;345:35-41.

[289] Chen H, Kim S, He W, Wang H, Low PS, Park K, et al. Fast release of lipophilic agents from circulating PEG-PDLLA micelles revealed by in vivo forster resonance energy transfer imaging. Langmuir. 2008;24:5213-7.

[290] Alexis F, Pridgen E, Molnar LK, Farokhzad OC. Factors affecting the clearance and biodistribution of polymeric nanoparticles. Mol Pharm. 2008;5:505-15. 
[291] Li W, Nakayama M, Akimoto J, Okano T. Effect of block composition of amphiphilic block copolymers on the physicochemical properties of polymeric micelles. Polymer. 2011;52:3783-90.

[292] Yang C, Attia AB, Tan JP, Ke X, Gao S, Hedrick JL, et al. The role of noncovalent interactions in anticancer drug loading and kinetic stability of polymeric micelles. Biomaterials. 2012;33:2971-9.

[293] Heinlein CA, Chang C. Androgen receptor in prostate cancer. Endocr Rev. 2004;25:276-308.

[294] So A, Gleave M, Hurtado-Col A, Nelson C. Mechanisms of the development of androgen independence in prostate cancer. World J Urol. 2005;23:1-9.

[295] Koivisto P, Kononen J, Palmberg C, Tammela T, Hyytinen E, Isola J, et al. Androgen receptor gene amplification: a possible molecular mechanism for androgen deprivation therapy failure in prostate cancer. Cancer Res. 1997;57:3149.

[296] Visakorpi T, Hyytinen E, Koivisto P, Tanner M, Keinanen R, Palmberg C, et al. In vivo amplification of the androgen receptor gene and progression of human prostate cancer. Nat Genet. 1995;9:401-6.

[297] Kallioniemi OP, Visakorpi T. Genetic basis and clonal evolution of human prostate cancer. Adv Cancer Res. 1996;68:225-55.

[298] Evan G, Littlewood T. A matter of life and cell death. Science. 1998;281:131722.

[299] Evan GI, Vousden KH. Proliferation, cell cycle and apoptosis in cancer. Nature. 2001;411:342-8.

[300] Shiozaki EN, Chai J, Rigotti DJ, Riedl SJ, Li P, Srinivasula SM, et al. Mechanism of XIAP-mediated inhibition of caspase-9. Mol Cell. 2003;11:519-27.

[301] Sasaki H, Sheng Y, Kotsuji F, Tsang BK. Down-regulation of X-linked inhibitor of apoptosis protein induces apoptosis in chemoresistant human ovarian cancer cells. Cancer Res. 2000;60:5659-66.

[302] Holcik M, Yeh C, Korneluk RG, Chow T. Translational upregulation of X-linked inhibitor of apoptosis (XIAP) increases resistance to radiation induced cell death. Oncogene. 2000;19:4174-7.

[303] Chawla-Sarkar M, Bae SI, Reu FJ, Jacobs BS, Lindner DJ, Borden EC. Downregulation of Bcl-2, FLIP or IAPs (XIAP and survivin) by siRNAs sensitizes resistant melanoma cells to Apo2L/TRAIL-induced apoptosis. Cell Death Differ. 2004;11:915-23. 
[304] McManus DC, Lefebvre CA, Cherton-Horvat G, St-Jean M, Kandimalla ER, Agrawal S, et al. Loss of XIAP protein expression by RNAi and antisense approaches sensitizes cancer cells to functionally diverse chemotherapeutics. Oncogene. 2004;23:8105-17.

[305] Fire A, Xu S, Montgomery MK, Kostas SA, Driver SE, Mello CC. Potent and specific genetic interference by double-stranded RNA in Caenorhabditis elegans. Nature. 1998;391:806-11.

[306] Singh S, Narang AS, Mahato RI. Subcellular Fate and Off-Target Effects of siRNA, shRNA, and miRNA. Pharmaceutical research. 2011;28:2996-3015.

[307] Reynolds A, Leake D, Boese Q, Scaringe S, Marshall WS, Khvorova A. Rational siRNA design for RNA interference. Nature biotechnology. 2004;22:326-30.

[308] Aigner A. Delivery systems for the direct application of siRNAs to induce RNA interference (RNAi) in vivo. Journal of biomedicine \& biotechnology. 2006;2006:71659.

[309] Huggins C, Hodges CV. Studies on prostatic cancer I: the effect of castration, of estrogen and of androgen injection on serum phosphatases in metastatic carcinoma of the prostate. Cancer Research. 1941;19:293-7.

[310] Huggins C, Hodges CV. Studies on prostatic cancer II: the effects of castration on advanced carcinoma of the prostate gland. Archives of Surgery. 1941;43:209-23.

[311] Danquah M, Fujiwara T, Mahato RI. Lactic Acid and Carbonate-based Crosslinked Polymeric Micelles for Drug Delivery. Journal of Polymer Science Part A: Polymer Chemistry. 2012: Accepted for Publication. 


\section{VITA}

Michael Danquah was born in Accra, Ghana, in 1980 to George Danquah and Elizabeth Danquah. Upon graduating with distinction from Presbyterian Boys' Senior Secondary School (PRESEC), Legon, Ghana in 1997, he enrolled in Kwame Nkrumah University of Science and Technology. There he pursued a Bachelor of Science degree in Chemical Engineering, graduating with First Class Honors in July of 2003. He then entered The Graduate School at The University of Kentucky in the fall of 2005 where he obtained a Master's degree in Chemical Engineering under the supervision of Professor Douglass S. Kalika in 2007. Subsequently, he joined the graduate program in the Department of Pharmaceutical Sciences at The University of Tennessee Health Science Center, Memphis, in the fall of 2007, where he was awarded a Ph.D. in Pharmaceutics (2012) under the mentorship of Professor Ram I. Mahato. He is now an Assistant Professor of Pharmaceutics at Chicago State University. 